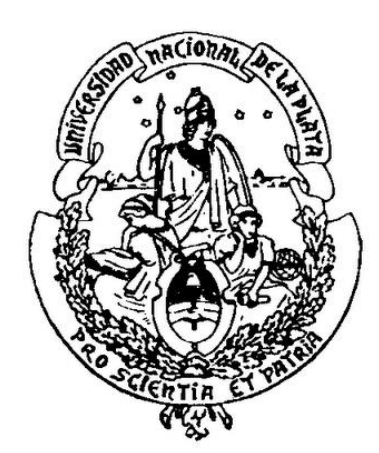

UNIVERSIDAD NACIONAL DE LA PLATA

FACULTAD DE CIENCIAS EXACTAS

DEPARTAMENTO DE QUÍMICA

Trabajo de Tesis Doctoral

\title{
DESARROLLO DE NANOMATERIALES BASADOS EN SILICIO SEMICONDUCTOR
}

\section{Juan J. Romero}

Directora: Dra. Mónica C. González

Co-director: Dr. Pedro F. Aramendía

\author{
La Plata - Argentina \\ Año 2017
}


El presente trabajo se llevó a cabo en el Instituto de Investigaciones Fisicoquímicas Teóricas y Aplicadas (INIFTA) del Departamento de Química de la Facultad de Ciencias Exactas, Universidad Nacional de La Plata, bajo la dirección de la Prof. Dra. Mónica C. Gonzalez, y en el Instituto de Química Física de los Materiales, Medio Ambiente y Energía (INQUIMAE) del Departamento de Química Inorgánica y Química Física de la Facultad de Ciencias Exactas y Naturales, Universidad de Buenos Aires bajo la co-dirección del Prof. Dr. Pedro F. Aramendía. El mismo se presenta a consideración de las autoridades de la Facultad de Ciencias Exactas de la UNLP para acceder al grado académico de Doctor de la Facultad de Ciencias Exactas.

\section{Miembros del Jurado}

Dra. María Gabriela Lagorio

Dr. Franco Cabrerizo

Dr. Ezequiel Wolcan

Fecha de la disertación oral: 27 de Marzo del 2017, INIFTA, La Plata.

Lic. Juan J. Romero

e-mail: jromero@inifta.unlp.edu.ar 
A Tomás y Daia.

A mis viejos. 


\section{Agradecimientos}

"I get by with a Little help from my friends"

Lennon \& McCartney - The Beatles

El hecho de que esta tesis sea hoy una realidad es fruto del esfuerzo y colaboración de una infinidad de personas. Estén o no en esta lista, que es la mejor recopilación que pude armar, voy a estar agradecido con ustedes toda mi vida.

En primer lugar quiero agradecer a Mónica Gonzalez, quien dirigió este trabajo, mi tesina y me enseño gran parte de lo que sé sobre fisicoquímica. Por ser una jefa comprensiva, tolerante y siempre dispuesta, pero por sobre todas las cosas por ser una buena persona. Por apoyar mis ideas y proyectos, animarme a seguir avanzando, ayudar a ordenarme y por confiar en mí. Por corregir o encarrilarme cada vez que fue necesario y por la infinita paciencia al momento de escribir.

A Pedro Aramendía, mi codirector. Cuando elegimos a Pedro pensamos en mil aspectos científicos, sin embargo al finalizar esta tesis puedo decir que terminé aprendiendo sobre fotoquímica y calidez humana al precio de uno. Por cada visita al CIBION, la discusión de ideas y las larguísimas charlas sobre Boca.

Al momento de elegir directores uno coloca muchas cosas en la balanza, y creo que salí beneficiado.

A Daniel Mártire, por todos los comentarios y consejos tanto a nivel científico como humano. Gracias por conducir junto a Mónica un grupo en el cual se respira un aire nuevo todos los días.

A José Hodak y Hernán Rodríguez, que se interesaron en mi trabajo y me ayudaron desde el día en que los conocí. Gracias por poner las manos en los experimentos, y discutir los resultados como si fuéramos compañeros de tesis de años.

A Julio Azcárate y Aldo Rubert, por medir infinidad de muestras y estar siempre dispuestos a discutir los resultados, sean los que sean. Gracias por ser una fuente continua de inspiración para trabajar.

A la Prof. Dra. Carola Kryschi y todo su grupo, quienes me recibieron en la Friedrich-Alexander Unviersität y me guiaron por toda Alemania. En especial a Marc, Anja, Luis, Rubén y Fernando por hacerme sentir en casa.

A los profesores Dra. Ana Moore, Dr. Tom Moore y Dr. Devens Gust de Arizona State University, por confiar en mí y transmitir todo el conocimiento que pudieron en 6 meses. Lo que aprendí trabajando en su laboratorio es invaluable, desde lo científico hasta lo personal.

Al prof. Dr. Val Vullev, Eli, Maciek y Jill, con quienes compartí un mes increíble en University of California at Riverside. Gracias por confiar en mi desde el primer día, incluso para arreglar el Ti:S y reconfigurar los experimentos ópticos que tenían montados. Gracias por hacerme sentir un miembro más del Vullev Group.

A las instituciones que permitieron el desarrollo de este trabajo de tesis: El INIFTA, que fue mi segundo hogar en estos 5 años y a la Facultad de Ciencias Exactas de la UNLP, por brindar educación de calidad y defender la educación pública como estandarte. A su vez, este trabajo no podría haber avanzado sin la financiación de varias agencias: CONICET, DAAD, el programa BEC.AR y la comisión Fullbright.

A mis amigos de ASU, que estoy seguro que al menos una vez más nos vamos a cruzar: Matías, Jimena, Lina, César, Alejo, Azaris, Daniel, Cindy, Marely, Iolanda, Sneha, Bobby, Chelsea, Christa, Zack, Brian, Kirt y Josh. Gracias por los mates, cafés, cervezas, partidos de fútbol y mil recuerdos geniales. Por tomar cada momento en Tempe y hacerlo inolvidable. 
A todos los miembros del LEAR, que si bien funcionamos como dos grupos separados nos rehusamos a aceptarlo. A lo largo de estos años pasó mucha gente, y sin dudas de todos aprendí algo. En particular, agradezco a Manu y ML por guiarme en mis primeros pasos y a Cris, Fabio Bel, Frani, Laurita, Lu, Juaqui y Damián por cada mate, té o cerveza en los últimos años. Por ser mis amigos dentro y fuera del laboratorio, por enseñarme a ser un poco más tranquilo y equilibrado con el trabajo.

A toda la división Fisicoquímica de FCE - UNLP. Gracias por seguir enseñándome todos los días, y hacerlo con tantas ganas y dedicación. Gracias por estar siempre dispuestos a cambiar y a mejorar, por enseñarme a ser un docente comprometido. A todos los estudiantes que crucé en los distintos cursos donde colaboré, gracias por ser una fuente interminable de ideas nuevas.

A los químicos: Javi G, Javi L, Bruno, Agus, Fiore y Yani. Por cada charla de pasillo, préstamos de reactivos o festejos del aniversario de la desvinculación. Cada vez que veo una función de partición, me río y los recuerdo. Gracias por estos 10 AÑOS juntos en todas.

A la peña de los Jueves. Gracias por acortar la semana, por todas las discusiones, por ser mi familia en La Plata y por el peña-check de cada día.

A los gatos del Molino: Tury, Guz, Mel, Vani y Romi (la infiltrada). Gracias por los momentos compartidos, desde aquellas rateadas hasta las horas largas hablando de la vida y de sueños.

A Benji, el Viejo, el Chino, D'Uva y el Gordo. Por ser mis amigos incansables, por estar en cada momento desde Jardín de Infantes. Los 5 juntos, en todas y en todo. Gracias por enseñarme que no hay distancia ni momento que pueda parar esta fiebre, ni si quiera estar del otro lado de la vida.

A Hec, Ren y Gaga, por cada abrazo, asado y vino que compartimos. Por recordarme de dónde vengo y a dónde quiero ir. Gracias por ser la banda de mi calle.

Al Grupo Scout Papa Francisco, por compartir un sueño y trabajar para cumplirlo. Gracias por todos los viajes, campamentos, módulos, consejos y proyectos. En especial a Fer, Maqui y Adrian, a quienes admiro profundamente, por compartir su forma de vivir la vida y construir juntos un mundo distinto. A los pibes, quienes nos marcan el camino y nos muestran que lo mejor está por venir.

A mi familia extendida: todos los Romero, Benitez, Schiavoni y Jaquier, que con detalles simples me apoyan a cada paso. A mi abuela Teresa, al Gordo, Cabeza, mi padrino Charly y Rocío; que estén donde estén, sé que me acompañan y guían a cada paso, y me ayudan a seguir.

A mis viejos y a Juampi, quienes vieron crecer este sueño y lo apoyaron desde que me inscribí hasta el día de hoy. Son la palara justa, el abrazo incansable y el aliento antes de salir a caminar.

Finalmente, con quienes recorremos juntos el camino: Tomi y Daia, quienes me llevan de la mano. Gracias por llenar de alegría y amor nuestros días, por el apoyo incansable y la fuerza a cada paso. Por confiar en mí, y construir juntos una vida llena de felicidad. Por ser la familia que siempre soñé, y que día a día se transforma en realidad.

GRACIAS

Juan J. Romero

La Plata, Marzo de 2017 
- 7 - 
Índice

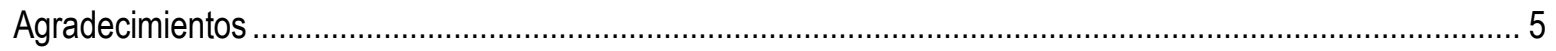

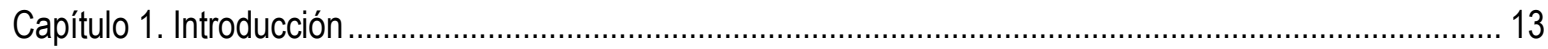

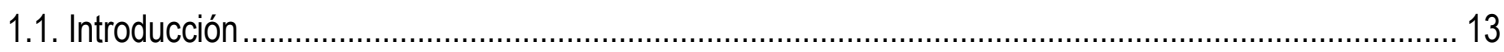

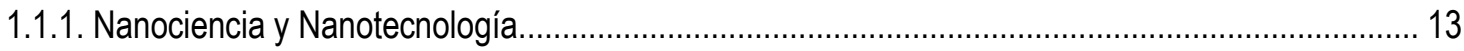

1.1.2. Aplicaciones de la Nanotecnología ............................................................................... 13

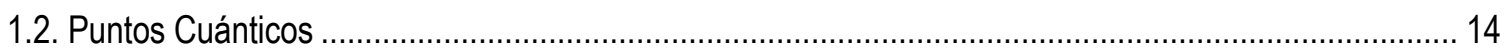

1.2.1. Elementos Semiconductores......................................................................................... 14

1.2.2. Efecto del Tamaño ………………………………………………………………………. 17

1.2.3. Estados excitados ................................................................................................................ 18

1.3. Nanopartículas de Silicio (Si nps) ................................................................................... 19

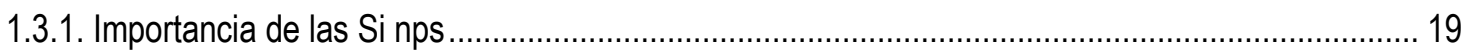

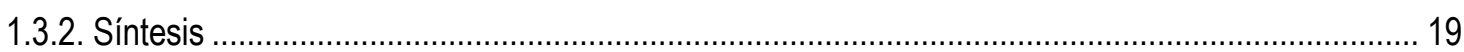

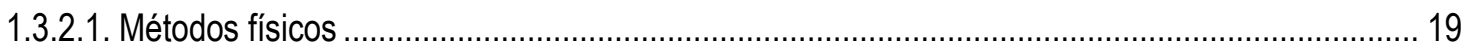

1.3.2.2. Métodos químicos de "arriba hacia abajo" ..................................................................... 20

1.3.2.3. Métodos químicos de "abajo hacia arriba" ....................................................................... 20

1.3.3. Modificación superficial .................................................................................................... 20

1.3.4. Propiedades fotofísicas ............................................................................................. 22

1.3.4.1. Funcionalización superficial y propiedades fotoluminiscentes ............................................... 22

1.3.5. Potenciales Aplicaciones como Marcadores Biológicos............................................................... 23

1.3.6. Potenciales Aplicaciones en Terapias Fotodinámicas .............................................................. 24

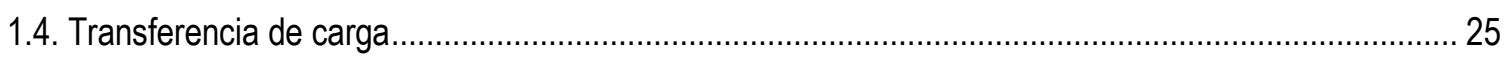

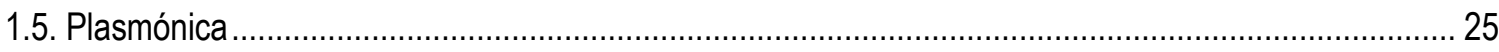

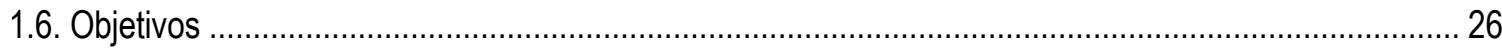

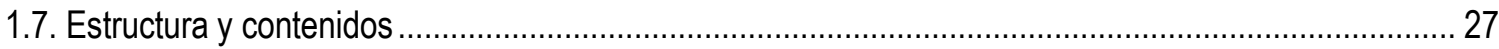

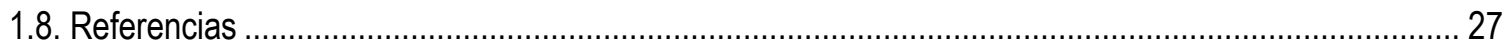

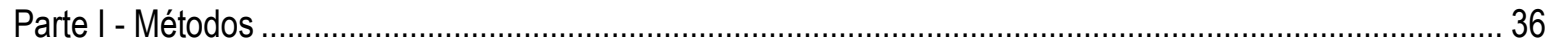

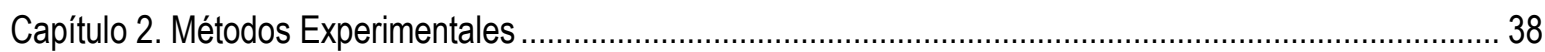

2.1. Espectroscopía estacionaria de absorción UV-Visible ............................................................... 38

2.2. Espectroscopía estacionaria de absorción en el IR ................................................................... 39

2.3. Voltamperometría cíclica .......................................................................................................... 40 


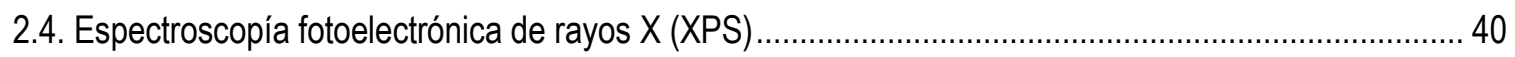

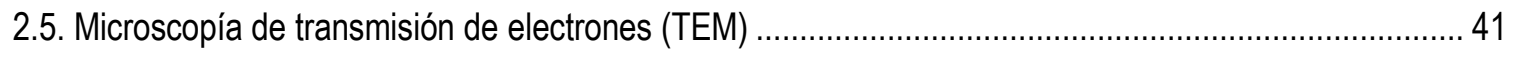

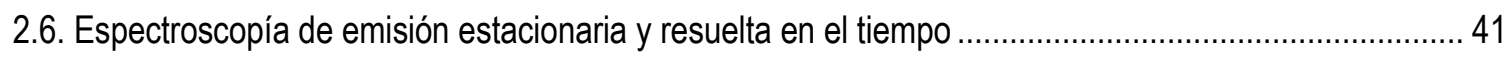

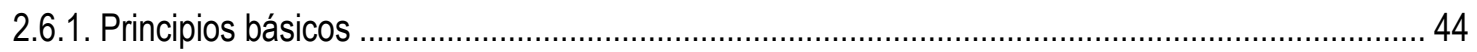

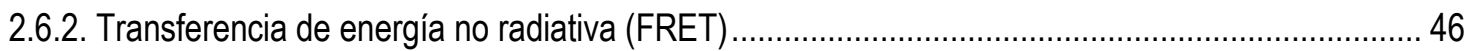

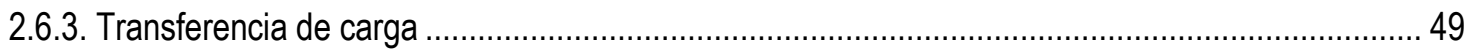

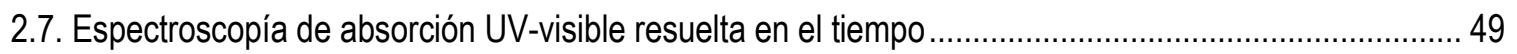

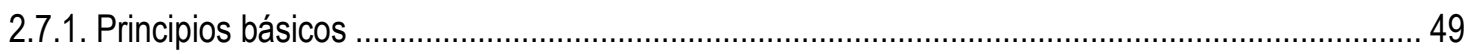

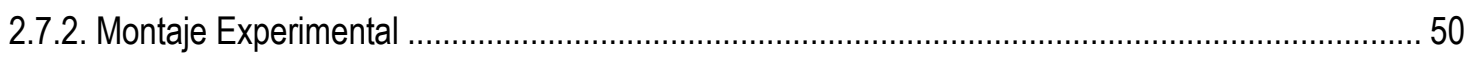

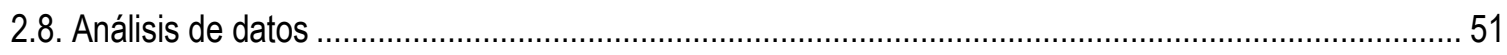

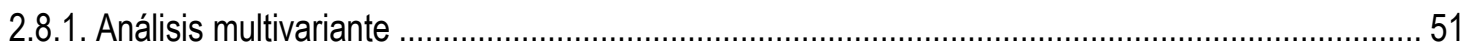

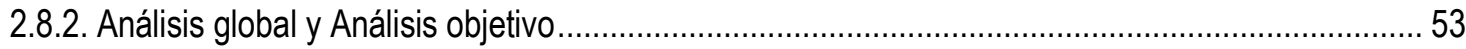

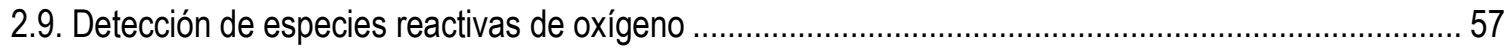

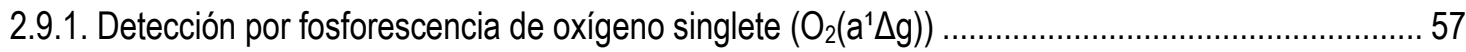

2.9.2. Detección de $\mathrm{O}_{2}\left(\mathrm{a}^{1} \Delta \mathrm{g}\right)$ mediante un Electrodo Sensible de $\mathrm{O}_{2}$ molecular .................................... 57

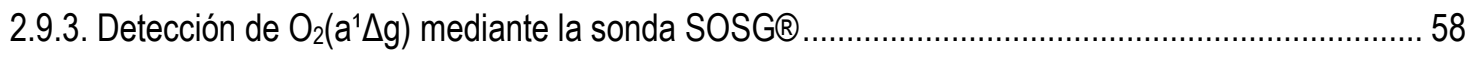

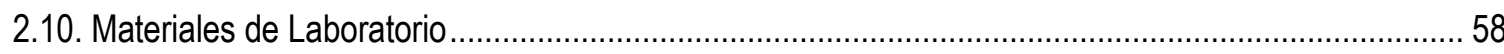

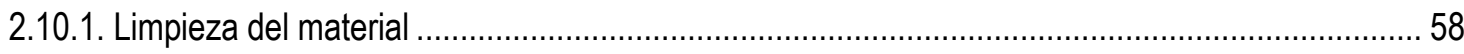

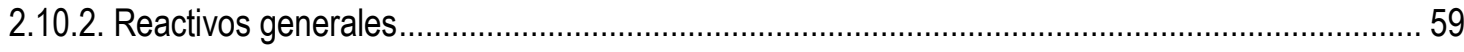

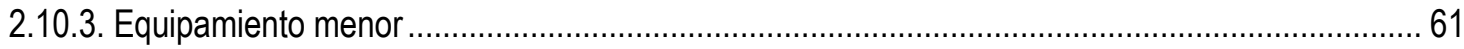

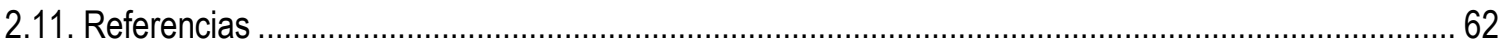

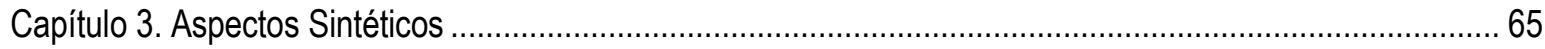

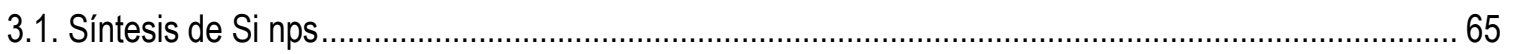

3.1.1. Síntesis de Si nps de $<d>=(3 \pm 1)$ nm por grabado electroquímico (TD' Si nps) ......................... 65

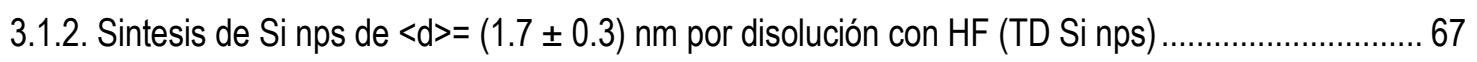

3.1.3. Síntesis de Si nps de $\langle d\rangle=(1.7 \pm 0.8) \mathrm{nm}$ por reducción en micelas inversas (BU Si nps) .......... 68

3.1.4. Sintesis de Si nps de $<d>=(3.4 \pm 0.6) \mathrm{nm}$ por oxidación en fase líquida (BU' Si nps $-\mathrm{S} 1) \ldots \ldots . . .69$

3.1.5. Sintesis de Si nps de $<\mathrm{d}>=(4 \pm 1) \mathrm{nm}$ por oxidación en fase líquida (BU' Si nps $-\mathrm{S} 4) \ldots \ldots \ldots \ldots . . . . . .69$

3.1.6. Sintesis de Si nps de $<\mathrm{d}>=(5 \pm 1) \mathrm{nm}$ por oxidación en fase líquida (BU' Si nps $-\mathrm{S} 3) \ldots \ldots \ldots \ldots \ldots . . . . .69$

3.1.7. Sintesis de Si nps de $<d>=(3.2 \pm 0.5)$ nm por oxidación en fase líquida (BU' Si nps $-\mathrm{SC}$ ) ......... 69

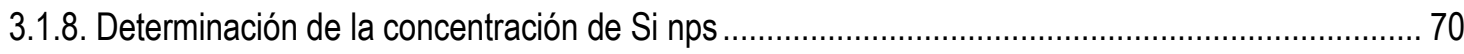

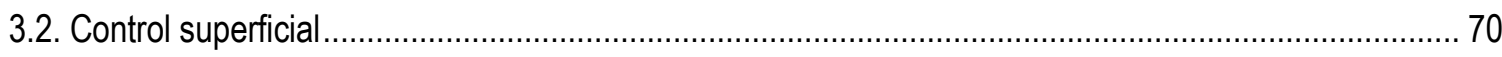

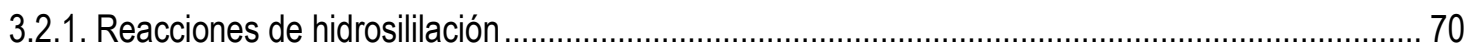


3.2.2. Reacciones de silanización

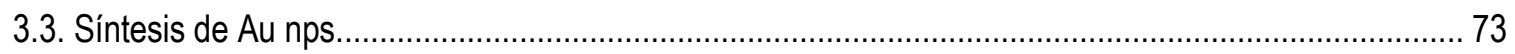

3.3.1. Síntesis de Au nps de $(22 \pm 3)$ por el método de Turkecivh - Frens (Au 32) ............................. 73

3.3.2. Síntesis de Au nps de $(68 \pm 4)$ por el método de crecimiento de semillas (Au 56) ........................ 73

3.3.3. Síntesis de Au nps de $(110 \pm 13)$ por el método de crecimiento de semillas (Au 87) .................... 73

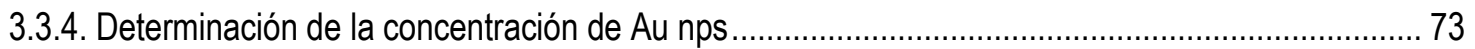

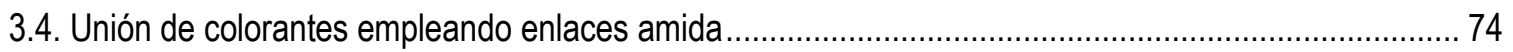

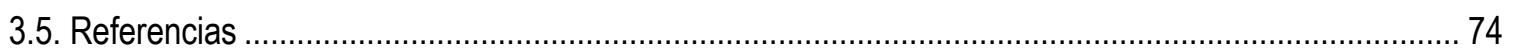

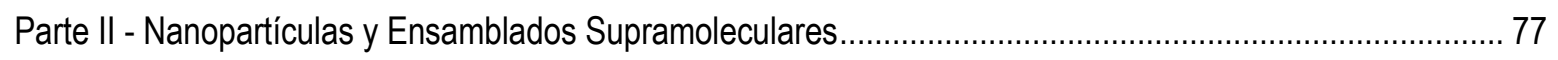

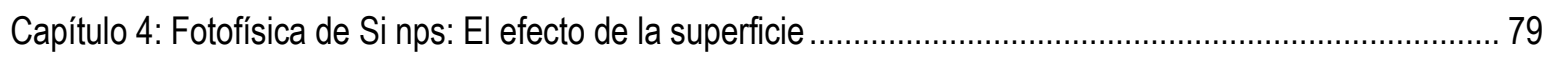

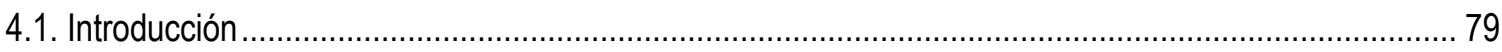

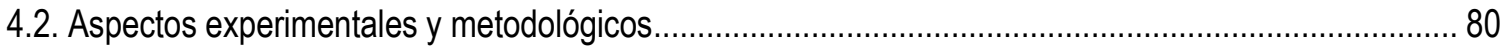

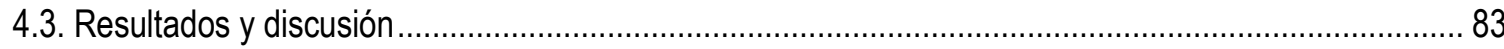

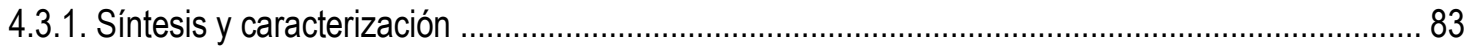

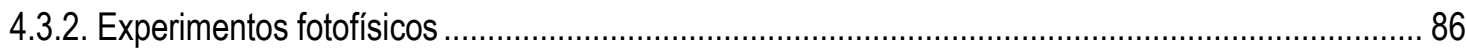

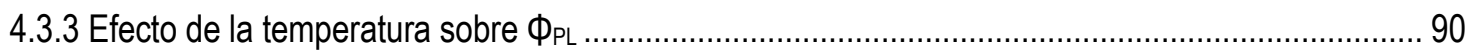

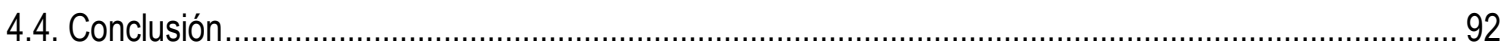

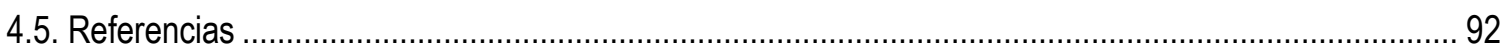

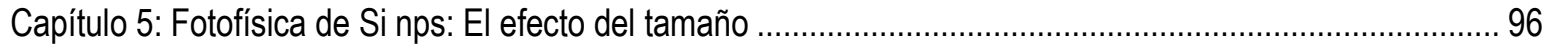

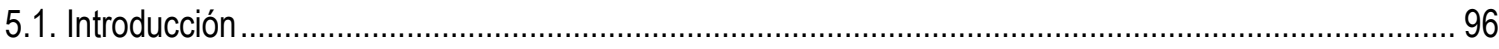

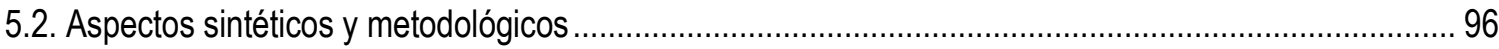

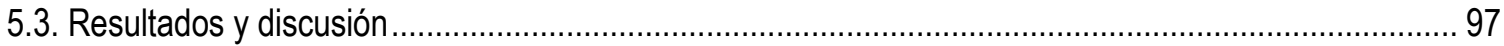

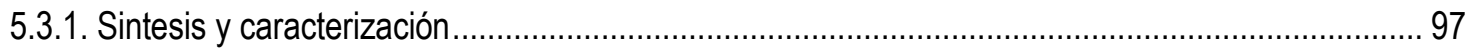

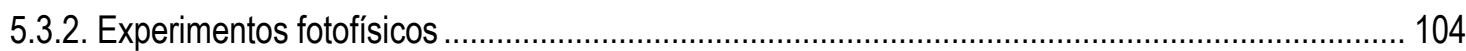

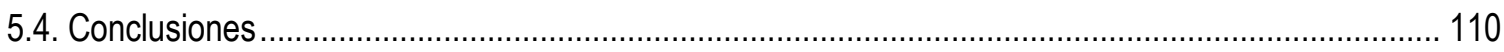

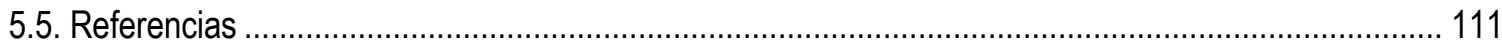

Capítulo 6: Regulación de propiedades ópticas. Díadas empleando colorantes orgánicos ......................... 115

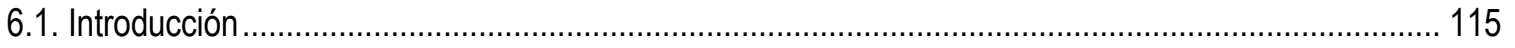

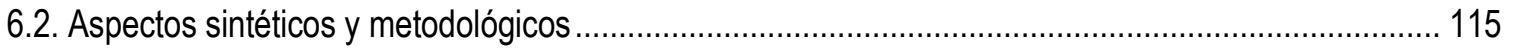

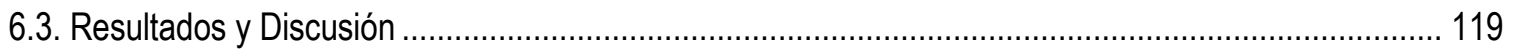

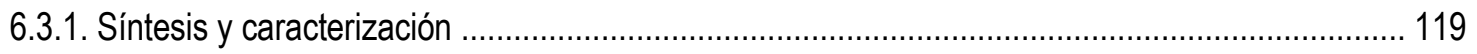

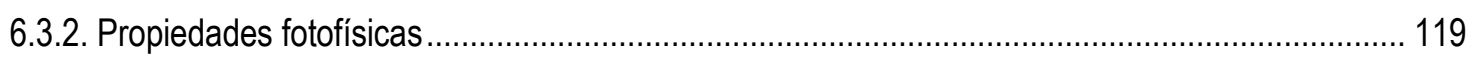

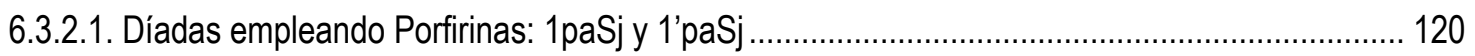




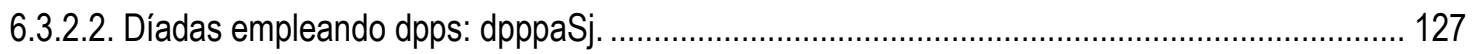

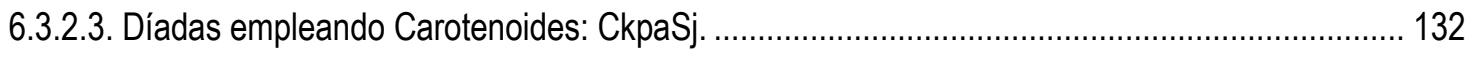

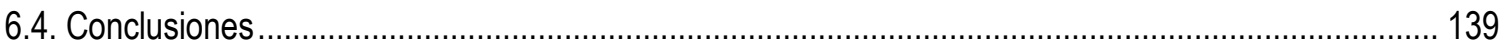

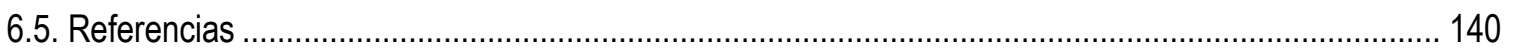

Capítulo 7: Regulación de propiedades ópticas. Ensamblados coloidales con Au nps ................................ 146

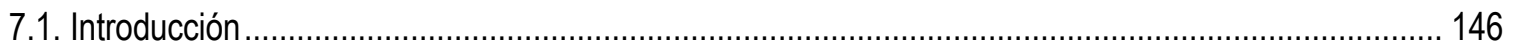

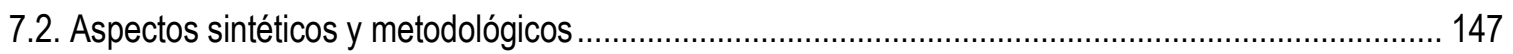

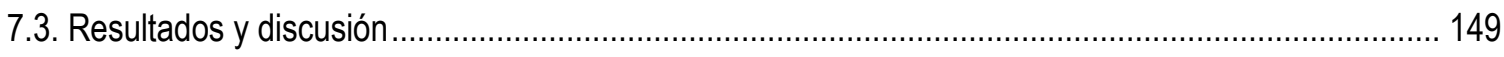

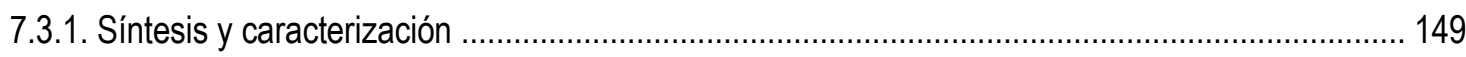

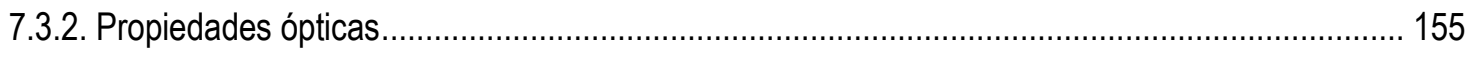

7.3.3. Generación de especies reactivas de oxígeno: Oxígeno Singlete $\mathrm{O}_{2}\left({ }^{1} \Delta_{g}\right) \ldots \ldots \ldots \ldots \ldots \ldots \ldots \ldots \ldots \ldots . . . . . . . . . . . . . . . . . .158$

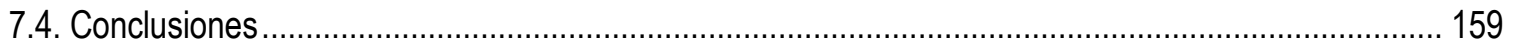

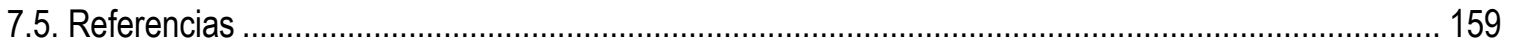

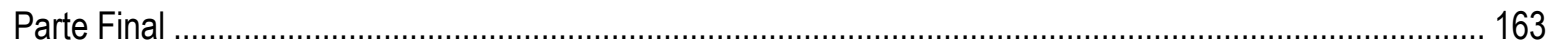

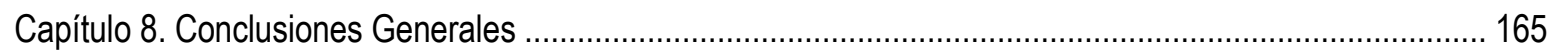

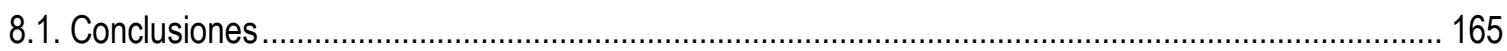

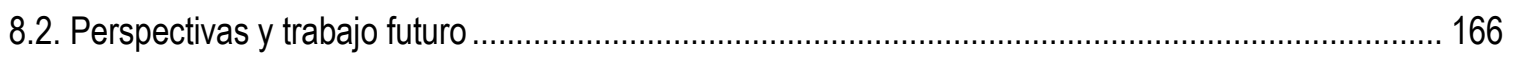

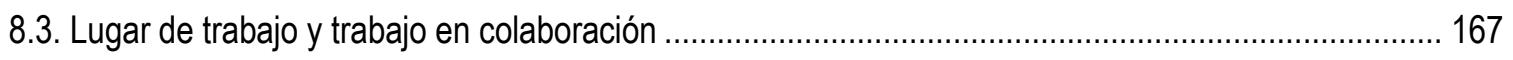

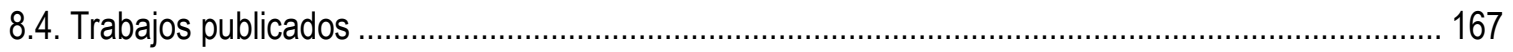

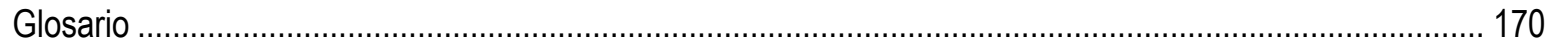


- 12 - 


\section{Capítulo 1. Introducción}

\subsection{Introducción}

\subsubsection{Nanociencia y Nanotecnología}

La nanociencia y la nanotecnología constituyen un campo interdisciplinario que incluye el estudio, diseño, creación, síntesis, manipulación y aplicación de materiales, aparatos y sistemas funcionales que posean al menos una dimensión espacial en el rango de los nanómetros $\left(1 \mathrm{~nm}=10^{-9} \mathrm{~m}\right)$. En estos tamaños los efectos de la mecánica cuántica y los fenómenos de superficie adquieren gran relevancia y dotan a los materiales de nuevas propiedades y características. La nanotecnología es un área de gran diversidad que engloba desde la modificación de dispositivos físicos convencionales hasta la generación de nuevos dispositivos basados en los fenómenos que se producen exclusivamente en la nanoescala.

Si bien el interés científico en esta área comenzó en 1960 [1] y estalló en la década de 1980 con la creación de las microscopías de barrido por sondas, las estructuras nanométricas existen en la naturaleza mucho antes que los científicos comenzáramos a estudiarlas: Una hebra de DNA, que es un bloque de construcción de toda la vida en la tierra, es de alrededor de $3 \mathrm{~nm}$ de largo. Los colores brillantes en las alas de las mariposas se deben a estructuras nanométricas que interactúan con la luz dando origen a estos colores. Los anillos coloreados que vemos en las burbujas de jabón también se originan en la interacción de la luz con estructuras de unos pocos nanómetros. Estos hechos nos dan una pauta importante al momento de investigar en este campo, que constituye una guía de acción: Los científicos creamos en el laboratorio estructuras que imitan las que existen previamente en la naturaleza. Si observamos y aprendemos de ella, nuestros diseños serán tanto más útiles y funcionales.

La concepción inicial de nanotecnología se refería siempre a la capacidad de crear estructuras construyendo dispositivos átomo a átomo, mediante técnicas que hoy denominamos "de abajo hacia arriba" (BU, del inglés bottom - up) para obtener productos con altas prestaciones controlando su diseño con precisión atómica. En la actualidad, la nanotecnología incorpora también las estructuras generadas a partir de materiales a granel reduciendo su tamaño mediante distintos métodos, conocidos como procesos "de arriba hacia abajo" (TD, del inglés top-down). Por lo tanto, queda bajo el término de nanotecnología cualquier tipo de estructura sintetizada tanto por métodos bottom-up o top-down que tenga un tamaño inferior a los $100 \mathrm{~nm}$ y muestre propiedades novedosas [2].

\subsubsection{Aplicaciones de la Nanotecnología}

La revolución originada por los dispositivos nanométricos es debida a la aparición de un gran número de nuevos fenómenos al reducir el tamaño de algunos materiales, entre los cuales se incluyen efectos de mecánica cuántica como el confinamiento cuántico[3], [4]. Propiedades que no se manifiestan en tamaños micrométricos, llegan a ser dominantes cuando el tamaño alcanza el rango de los nanómetros. Adicionalmente, un gran número de propiedades mecánicas, eléctricas y/u ópticas cambian cuando se compara con los correspondientes sistemas macroscópicos. Ejemplos de estas propiedades emergentes son el cambio de color, como en el caso del Oro y Plata [5][6] o la emisión eficiente de radiación electromagnética luego de una excitación, como en el caso del Silicio [7].

La amplitud de enfoques que existen dentro de la nanotecnología permitieron el desarrollo de diversos subcampos como la bionanotecnología, que aborda el diseño y síntesis de sistemas nanoestructurados para imitar o incorporar en sistemas biológicos a nivel molecular, o construir diminutas herramientas para estudiar el cambio de las propiedades estructurales átomo por átomo, tal y como sucede en sistemas vivos. Algunas de 
las aplicaciones que se desarrollan en la actualidad son biodetección de patógenos, detección de proteínas, determinación de estructuras de DNA, ingeniería de tejidos, destrucción de tumores por calor, estudios patogénicos, separación de biomoléculas y células, marcadores biológicos fluorescentes, transporte de medicamentos, células artificiales, diseño de proteínas, formación y crecimiento de nanoestructuras en sistemas vivos o biosensores [8].

Las nanopartículas (nps), que poseen sus tres dimensiones espaciales menores que $100 \mathrm{~nm}$ constituyen un área de intensa investigación científica, debido a la amplia variedad de aplicaciones potenciales en campos biomédicos, ópticos, electrónicos, nanoquímica, o agricultura [9]-[11]. En particular, los resultados más sorprendentes se obtuvieron a partir de las nps construidas a partir de metales nobles y de semiconductores. En el primer caso, la aparición de una banda de absorción (llamada banda del plasmón superficial SPB, del inglés Surface Plasmon Band) sensible tanto al tamaño de las nps como al entorno dieléctrico, y en el segundo la posibilidad de ajustar las propiedades de emisión de radiación electromagnética ajustando su tamaño.

Algunas de las propiedades de las nanopartículas metálicas son conocidas desde la antigüedad y existen informes sobre sus usos desde el siglo XVII [12]. No obstante, en $1857 \mathrm{M}$. Faraday disertó acerca del oro coloidal generado por reducción de sales de Au, se considera un hito en la historia de las Au nps. Casi un siglo después de la conferencia de Faraday, J. Turkevich publicó el método de síntesis de Au nps protegidas por citrato [5], que se utiliza extensamente hasta la actualidad. La investigación de la composición y estructura de las nps es interesante tanto para poder comprender mejor la variación de distintos fenómenos físicos por la restricción del tamaño a la escala nanométrica como así también por la gran variedad de aplicaciones que estas poseen en campos tan diversos como el tratamiento médico, diagnóstico en química clínica, catálisis y fabricación de sensores y/o biosensores [13][12][14].

En el caso de las nanopartículas de materiales semiconductores, conocidos como puntos cuánticos (QDs, del inglés Quantum Dots), el interés en sus propiedades electrónicas aumentó notablemente en la década de 1990 [3], [4], [15], cuando los métodos de síntesis en fase líquida a partir de precursores simples de los grupos II y VI A fueron estudiados en forma exhaustiva. Este trabajo condujo a la posibilidad de controlar el tamaño de los QDs a partir de variables sintéticas fáciles de controlar, y en consecuencia permitió explorar de forma precisa el efecto del tamaño en la dinámica del proceso de emisión [16]-[19]. A partir de los avances realizados fue posible comenzar a trabajar en aplicaciones en nanomedicina [20], conversión de energía [21], censado [22] y catálisis [23][24].

Uno de los objetivos finales de la nanotecnología, es la integración de los bloques de construcción nanométricos en sistemas funcionales. Una forma de lograrlo es a través del autoensamblado dirigido por interacciones no covalentes y la química supramolecular [25]-[29]. Esta vía se manifiesta en sistemas biológicos diversos y constituye el camino a seguir para construir sistemas estables [30]-[32]. A través de esta estrategia se diseñaron cristales fotónicos para manipulación óptica [7], [33], celdas fotoelectroquímicas para conversión de energía [21][34], sistemas que imitan el fotosistema II del aparato fotosintético [35], [36], o sistemas responsivos al entorno en el cual se encuentran [37]. En los últimos años, la síntesis de estructuras supramoleculares con propiedades nuevas que emergen del acoplamiento de los distintos bloques de construcción nanométricos están redefiniendo las nuevas fronteras en la ciencia de los materiales [38].

\subsection{Puntos Cuánticos}

\subsubsection{Elementos Semiconductores}

Los sólidos cristalinos se dividen en función de su conductividad eléctrica en tres clases: metales, semiconductores y aislantes, en particular los semiconductores poseen una conductividad eléctrica intermedia. Cuando estos materiales e encuentran en fase masiva, los estados electrónicos presentan niveles energéticos muy cercanos tal que se genera un cuasi-continuo de estados denominados bandas. El concepto de bandas en sólidos es análogo al de orbitales moleculares en moléculas [39][40], [41]. En este marco, la última banda 
ocupada se llama banda de valencia (VB, del inglés Valence Band) mientras que la primera banda vacía es la banda de conducción ( $\mathrm{CB}$, del inglés Conduction Band). A su vez, entre estas bandas hay un intervalo energético donde no existen estados electrónicos, con lo cual un electrón no puede tomar valores de energía en dicho intervalo. Este espacio se llama banda prohibida o Band Gap (BG), análogo a la diferencia de energía entre el HOMO - LUMO para pequeñas moléculas. Volviendo a la primera clasificación que realizamos para sólidos cristalinos, pero desde la óptica de la teoría de bandas, los buenos conductores eléctricos se caracterizan por tener un BG nulo o muy pequeño, tal que a temperatura constante las bandas de valencia y conducción se solapen y permitan la población de CB, cuyos electrones son los responsables de la conducción eléctrica. En el caso de aislantes, BG es lo suficientemente grande tal que, a una temperatura dada, la población de electrones en la CB es nula. Los semiconductores tienen la VB llena separada de la CB (en el estado fundamental vacía) por una BG intermedio al caso de conductores y aislantes. El Band Gap para semiconductores está entre 0,5 y 3,5 eV, siendo en particular el del silicio de 1,2 eV y por tanto solo absorbiendo radiación con longitudes de onda $\lambda<1100 \mathrm{~nm}$ [7] puede poblarse la CB. El BG juega un rol muy importante en la fotofísica de semiconductores, ya que determina en que región del espectro electromagnético el material puede absorben energía a través de radiación.

Para hacer un análisis más detallado del sistema en cuestión, es conveniente considerar a los electrones dentro del sólido moviéndose libre e independientemente, siendo este sólido una caja de lados $L_{x}, L_{y}$ y $L_{z}$ con un vértice en $(x, y, z)=(0,0,0)$. El potencial para este sistema es:

$$
V(x, y, z)=\left\{\begin{array}{c}
0 \text { si } 0<x<L_{x}, 0<y<L_{y}, 0<z<L_{z} \\
\infty \text { en cualquier otro caso }
\end{array}\right.
$$

Si bien es un modelo sumamente rudimentario [40][41], es de utilidad para elucidar los principios básicos de las propiedades en consideración.

El Hamiltoniano para el sistema de $\mathrm{N}$ electrones, bajo las suposiciones realizadas, es suma de $\mathrm{N}$ Hamiltonianos monoelectrónicos. Por lo tanto, la ecuación de Schrödinger para el sistema es la siguiente:

$$
\left(\frac{-\hbar^{2}}{2 m} \sum_{i=1}^{N} \nabla_{i}^{2}+V(x, y, z)\right) \Psi\left(x_{1}, y_{1}, z_{1}, \ldots, x_{N}, y_{N}, z_{N}\right)=E \Psi\left(x_{1}, y_{1}, z_{1}, \ldots, x_{N}, y_{N}, z_{N}\right)
$$

Siendo $\hbar$ la constante reducida de Planck y $m$ la masa del electrón en la estructura cristalina. A su vez, como los electrones son independientes, la función de onda plurielectrónica que caracteriza al sistema puede aproximarse como un producto de funciones monoelectrónicas. Teniendo esto en cuenta, la ecuación (1.1) puede desdoblarse en $\mathrm{N}$ ecuaciones para un electrón:

$$
\left(\frac{-\hbar^{2}}{2 m} \nabla_{i}^{2}+V(x, y, z)\right) \varphi(x, y, z)=\varepsilon_{i} \varphi(x, y, z)
$$

Teniendo en cuenta la periodicidad del sistema, las condiciones de borde para estas ecuaciones son de la siguiente forma:

$$
\left\{\begin{array}{l}
\varphi(0, y, z)=\varphi\left(L_{x}, y, z\right) \\
\varphi(x, 0, z)=\varphi\left(x, L_{y}, z\right) \\
\varphi(x, y, 0)=\varphi\left(x, y, L_{z}\right)
\end{array}\right.
$$


Siendo $L_{x}, L_{y}$ y $L_{z}$ las longitudes características del sistema en la dirección de los ejes $x, y, y z$ respectivamente. Al resolver la ecuación monoelectrónica, se obtiene un conjunto de estados con energía $E$ caracterizados por un vector de onda $\mathbf{k}$, cuyo valor se encuentra cuantizado. Este vector se relaciona con el momento del cristal, según las siguientes ecuaciones:

$$
E=\frac{\hbar^{2} k^{2}}{2 m} \quad \wedge \overrightarrow{\mathrm{k}}=\pi\left(\frac{n_{x}}{L_{x}} \mathbf{i}+\frac{n_{y}}{L_{y}} \mathbf{j}+\frac{n_{x}}{L_{x}} \mathbf{k}\right) \quad \overrightarrow{\mathrm{p}}_{c}=\hbar \overrightarrow{\mathrm{k}} \quad \wedge
$$

La relación funcional entre $\mathbf{k}$ y la energía $\mathrm{E}$ de la banda es llamada relación de dispersión, y es característica de cada banda. La función $E(\mathbf{k})$ es el análogo en física del estado sólido a las superficies de energía potencial en estructura electrónica molecular. Para un sólido determinado, la colección de todas las funciones $E(\mathbf{k})$ constituyen la estructura de bandas del mismo.

Si tenemos en cuenta la estructura de bandas del sistema modelo considerado, vemos el siguiente comportamiento:

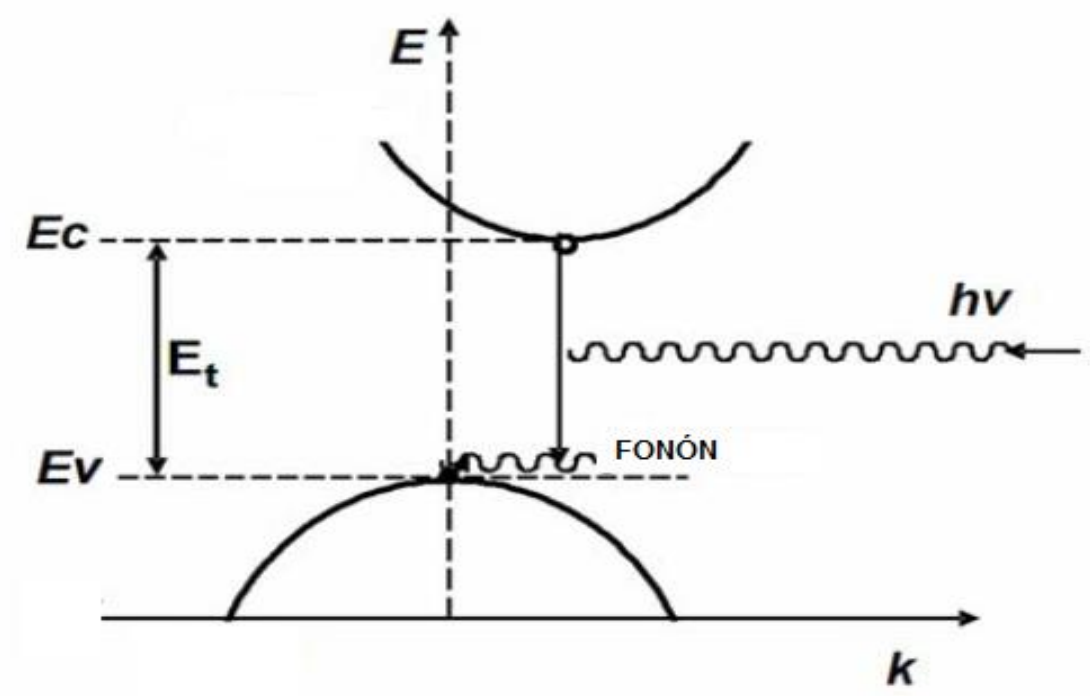

Figura 1.1. Comportamiento de la energía en función del vector de onda k (Esquema SOLAMENTE representativo).

Siendo $E_{c}$ la energía más baja de $C B, E_{v}$ la energía máxima de $V B$ y $E_{t}$ la diferencia de energía asociada a la transición entre estos 2 puntos. Finalmente, una transición electrónica entre dos estados estará más favorecida o no según el valor que tome el momento de transición entre el estado inicial y final $\langle\mathrm{f}|\boldsymbol{\mu}| \mathrm{i}\rangle=\boldsymbol{\mu}_{\mathrm{if}}$. Además, deben cumplirse los requerimientos de conservación de energía y momento. Ante esta situación, surgen 2 tipos de transiciones:

- Directas, donde los estados inicial y final poseen el mismo vector de onda $\mathbf{k}$, y por lo tanto el momento se conserva automáticamente. Estos procesos son eficientes y tienen tiempos característicos del orden de $10^{-9} \mathrm{~s}$.

- Indirectas, en caso de que los estados inicial y final tengan vectores de onda distintos. Entonces, para que se cumpla la conservación del momento y la transición sea observada, debe ser asistida por un fonón de la red. Debido a la menor probabilidad de que este tipo de transiciones ocurra, ya que involucra procesos acoplados, tienen tiempos característicos más largos, en el orden de los $10^{-6} \mathrm{~s} y$ poseen menores eficiencias, comparadas con transiciones directas. 
Si bien la probabilidad de ocurrencia de una transición indirecta es baja, los defectos en la red cristalina y de simetría pueden aumentar las probabilidades de estas transiciones. Cuando se irradia un semiconductor con fotones de mayor energía que $B G$ puede promoverse un electrón a la $\mathrm{CB}$, dejando una carga positiva denominada hueco en la VB. Este hueco se puede considerar como una partícula con masa efectiva y carga en el interior del sólido. Es posible calcular la separación espacial entre un electrón y su hueco (par llamado excitón) aproximando este par como un átomo hidrogenoide. Considerando el modelo de Bohr, obtenemos:

$$
r=\varepsilon h^{2} / \pi \mu e^{2}
$$

donde $r$ es el radio de la esfera definida por la separación tridimensional del par hueco-electrón; $\varepsilon$ es la permitividad eléctrica del medio, $\mu$ es la masa reducida del par hueco - electrón; $h$ es la constante de Planck y e es la carga de un electrón [40]. Para cada semiconductor hay una distancia característica de separación del par hueco - electrón que se llama radio de Bohr $r_{B}$, que en el caso del Silicio vale $4.5 \mathrm{~nm}$ [42]. Este valor indica el tamaño mínimo que debería presentar un cristal para poder ser tratado como un sólido en fase masiva y que su estructura electrónica pueda describirse apropiadamente a través de bandas electrónicas.

\subsubsection{Efecto del Tamaño}

Es conocido que los semiconductores II-VI manifiestan transiciones directas, mientras que el Silicio (Si) en fase masiva muestra transiciones indirectas. No obstante, en la escala nanométrica debemos tener en cuenta la naturaleza ondulatoria de la materia. Consideremos un electrón dentro de una caja, cuyas dimensiones en principio son macroscópicas. En estas condiciones, la posición de los mismos no puede ser fijada, por lo tanto, el momento puede definirse con cierta precisión. Al disminuir el tamaño de la caja a la escala de los nanómetros, la posición de los electrones se encuentra confinada, con lo cual el momento no puede ser fijado. Por lo tanto, al considerar transiciones en principio indirectas bajo el modelo de electrones libres e independientes, el principio de conservación del momento se relaja ${ }^{1}$ y las transiciones se comportan como directas [43].

La disminución del tamaño de un material semiconductor cristalino genera un cambio en la estructura electrónica del mismo, pasando del pseudo-continuo que se observaba en fase masiva a niveles de energía discretos en sistemas atómicos, lo cual conlleva a un cambio en la diferencia de energía entre el estado fundamental y los estados excitados, el cual varía desde BG para el material en fase masiva hasta la diferencia HOMO - LUMO en clusters de pocos átomos, sumado a las implicaciones del principio de incertidumbre sobre las propiedades de las transiciones electrónicas. Las partículas con tamaños menores que el radio de Bohr del excitón muestran un cambio hacia la estructura de niveles discretos, con diferencias de energía entre el estado fundamental y el excitado regulables por el tamaño de la partícula. Este efecto lleva a la posibilidad de regular bandas de absorción y/o emisión. A medida que las dimensiones del semiconductor se reducen hasta la escala nanométrica, el BG aumenta de forma significativa. En la figura 1.2 se muestra el efecto cualitativo de la variación del BG a medida que cambia el tamaño del agregado, mostrándose para cristales macroscópicos, nanopartículas de $5 \mathrm{~nm}$ y clusters de $1 \mathrm{~nm}$ [44], [45].

\footnotetext{
${ }^{1}$ Esta afirmación no debe ser mal interpretada, ya que la relajación de esta condición no implica la no conservación del momento, sino que indica que este principio se cumple sujeto a las restricciones que impone el principio de incertidumbre.
} 


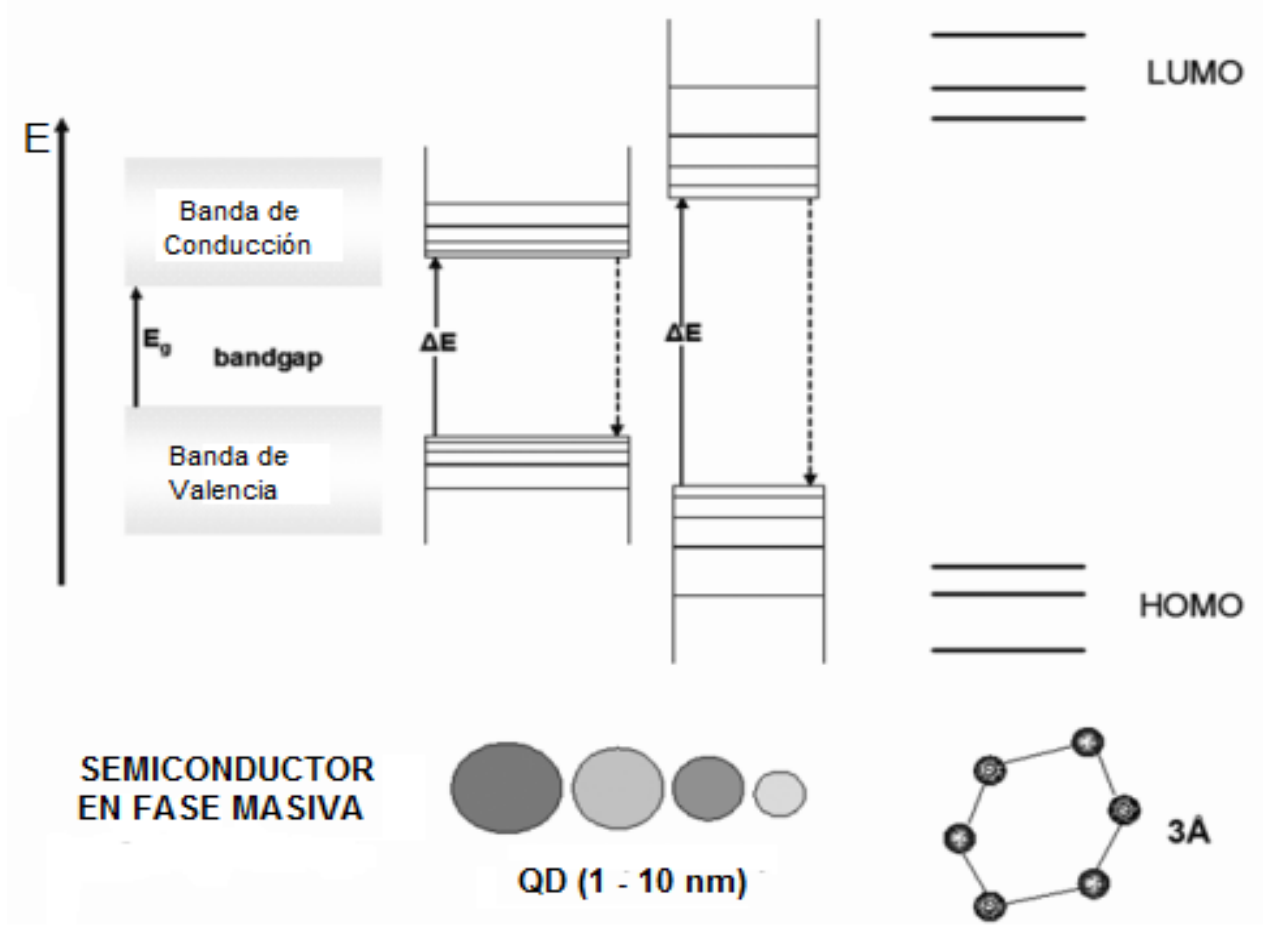

Figura 1.2. Variación de la estructura de niveles de energía en semiconductores en función del tamaño. Adaptado de [45].

En cristales de tamaños comparables al radio de Bohr las funciones de onda de los excitones están deslocalizadas sobre toda la partícula, conociéndose este efecto como confinamiento cuántico [4], [18], [46]. Cada material tiene un radio de Bohr del excitón característico y marca el tamaño máximo en el que se presenta el efecto de confinamiento cuántico. El confinamiento cuántico en semiconductores con de transiciones directas ha sido ampliamente estudiado [17], [19], [47], [48] y actualmente la comunidad científica trabaja en el desarrollo de aplicaciones tecnológicas basadas en los mismos.

\subsubsection{Estados excitados}

Los QDs generan luminiscencia por transiciones electrónicas al igual que los colorantes orgánicos tradicionales, con al menos dos grandes diferencias:

- Los electrones son promovidos de una banda de energía a otra, en vez de pasar de un nivel de energía discreto a otro,

- La superficie tiene un rol preponderante sobre las vías de relajación del QD.

Salvando estas diferencias, el destino de un estado excitado es similar al descripto par colorantes orgánicos tradicionales, experimentando canales de relajación unimoleculares radiativos (Luminiscencia) y no radiativos (conversión interna, conversión entre sistemas) o bimoleculares (transferencia de carga, energía, o formación de aductos). Las escalas de tiempo observadas están en el orden de lo reportado para colorantes orgánicos. 


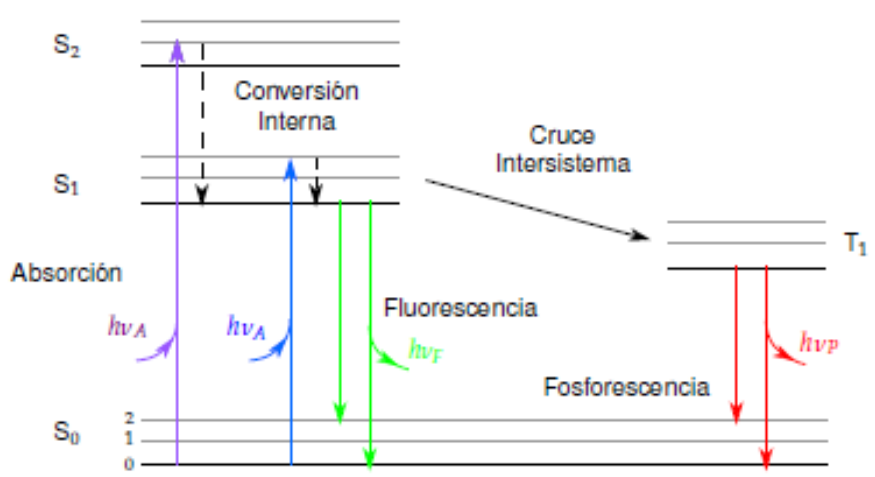

(a)

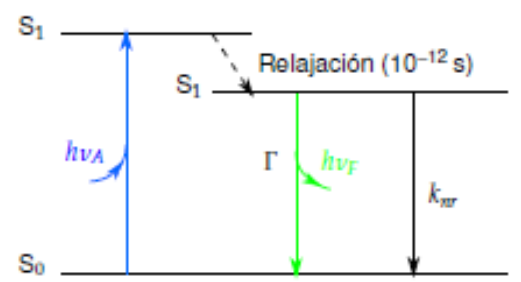

(b)

Figura 1.3. (a) Ejemplo de un diagrama de Jablonski, adaptado de la referencia [49], (b) diagrama de Jablonski simple donde solo se considera la fluorescencia.

\subsection{Nanopartículas de Silicio (Si nps)}

\subsubsection{Importancia de las Si nps}

Las numerosas publicaciones sobre QDs de semiconductores II - VI basados en Cd [3], [4], [45], [50] muestran un gran avance en la síntesis, logrando un gran control sobre el tamaño, forma y química superficial; lo cual permitió la comprensión de sus propiedades fundamentales. En la actualidad existen diversas aplicaciones de estas nanopartículas en diagnóstico médico, electrónica o conversión de energía solar [21]. Sin embargo, todas estas iniciativas se ven limitadas por la toxicidad intrínseca de los iones $\mathrm{Cd}^{2+}$. Este importante factor desfavorable incentivó a la comunidad científica a trabajar en QDs libres de Cd, usando semiconductores no tóxicos. Ante esta situación, el Silicio constituye una alternativa interesante, ya que es el segundo elemento más abundante en la superficie terrestre, existe toda una industria electrónica basada en él, su química está bien desarrollada y, sobre todo, es biológicamente compatible.

La fotoluminiscencia (PL, del inglés Photoluminescence) en nanoestructuras de silicio fue reportada por primera vez en el año 1990 por Canham para el caso de Silicio poroso anodizado [7]. A partir de este descubrimiento, los estudios sobre el silicio poroso y los cristales de silicio nanométricos han despertado el interés de la comunidad científica debido a sus propiedades fotofísicas únicas [45].

\subsubsection{Síntesis}

Existen diversas rutas sintéticas para obtener nanomateriales basados en $\mathrm{Si}$, como films nanoporosos y estructuras con porosidad jerárquica, nanohilos y nanopartículas de silicio, Si nps. Enfocándonos en Si nps, los métodos existentes pueden clasificarse como físicos y netamente químicos. Dentro de este último grupo, podemos dividirlos como técnicas de "arriba hacia abajo" o de "abajo hacia arriba": En el primero se parte de precursores moleculares de Si, que reaccionan y forman los clusters de Si por crecimiento; mientras que en el segundo grupo se parte de materiales macroscópicos que contengan Si para tratarlos por diversos ataques químicos y obtener estructuras nanométricas.

\subsubsection{Métodos físicos}

Dentro de las técnicas físicas para producir Si nps se emplean implantación de iones Si en films de $\mathrm{SiO}_{2}$ [51], [52], recocido a altas temperaturas de óxidos de Si [43], pirolisis de silanos [46] o ablación de blancos de Si usando láseres [53]. Estos métodos presentan dificultades particulares, como son la liberación de las Si nps de los films de implantación, tratamientos post-sintéticos y bajos rendimientos. 


\subsubsection{Métodos químicos de "arriba hacia abajo"}

Dentro de las vías químicas TD, el uso de reacciones electroquímicas sobre blancos de Si de alta pureza constituye el método predilecto, con variaciones sutiles que permiten controlar el tamaño, morfología, porosidad o estado de la superficie [42], [54], [55]. Esta técnica se basa en la disolución anódica de electrodos de Si en presencia de HF, en condiciones controladas de corriente y potencial, lo cual genera una red porosa en el ánodo de $\mathrm{Si}$, el cual posteriormente se somete a baño de ultrasonidos y desprende partículas nanométricas. La distribución de tamaños, estado de la superficie o morfología puede variarse cambiando la densidad de corriente, la composición del electrolito soporte o el tiempo de electrólisis [7]. El principal problema de estos métodos es por un lado el uso de HF, una sustancia altamente peligrosa, y por el otro la complicación para el escalado y la obtención de mayores cantidades de Si nps (en un procedimiento típico, se obtienen ng de Si nps).

\subsubsection{Métodos químicos de "abajo hacia arriba"}

Las técnicas BU implican el uso de precursores moleculares de Si en diversas reacciones químicas de óxido reducción que forman una nueva fase, la cual por el uso de diversos medios de reacción se restringe a cristales de tamaño nanométrico.

En los métodos que emplean fluidos supercríticos, diversos organosilanos pueden disolverse en algún solvente adecuado, y posteriormente son calentados y presurizados sobre el punto crítico de este último, para iniciar el crecimiento de la partícula por descomposición térmica del precursor que contiene Si. Así, pueden obtenerse Si NP relativamente monodispersas, pero con una composición superficial difícil de controlar. A su vez, el tamaño del agregado puede controlarse por el agregado de algún cosolvente, que haga disminuir la relación sustrato/solvente estabilizante [56], [57].

Para controlar de forma eficiente el tamaño de las Si nps sintetizadas, una opción viable es el empleo de micelas de algún surfactante adecuado como nano-reactores [58]. En general, se emplean compuestos del tipo SiX $(\mathrm{X}=\mathrm{Cl}, \mathrm{Br}$ o I), que se localizan en el interior hidrofílico de la micela, que por el agregado de un reductor originan Si nps cuyo crecimiento está restringido por el entorno micelar [59]-[61]. El tamaño de las nps puede controlarse limitando el tamaño de las micelas, interacciones intermicelares, etc.

Existen varias vías de síntesis en solución que emplean la oxidación o reducción de precursores del tipo fases de Zintl que contengan Si: Reducción de $\mathrm{SiCl}_{4}$ con NaSi o Naftalenuro de sodio, u oxidación de $\mathrm{Mg}_{2} \mathrm{Si}$ con $\mathrm{Br}_{2}$ [62]-[68]. Estos métodos permiten regular el tamaño de las NP mediante variaciones de temperatura a lo largo de la reacción, concentración de precursores, etc. Si bien este tipo de técnicas son muy versátiles y permiten no solo la obtención de Si nps, sino también nanopartículas de Germanio [68] e incluso nanopartículas dopadas [69], [70], las muestras son polidispersas y necesitan un mayor trabajo de purificación.

\subsubsection{Modificación superficial}

Las propiedades físicas y fotofísicas de Si nps dependen fuertemente de su composición superficial. El aumento de la relación superficie: volumen junto con la mayor reactividad comparado al Si en fase masiva (consecuencia de la alta curvatura de las superficies, que generan enlaces $\mathrm{Si}$ - Si más estirados, tensos y lábiles), hacen que estas Si nps sean muy sensibles tanto al agua como al aire. En el caso de superficies activas de Si nps (cubiertas con $\mathrm{H}$ o halógenos),se da una pasivación rápida formando una capa de óxido $\left(\mathrm{SiO}_{\mathrm{x}}\right)$ que tiene una fuerte influencia sobre los canales de decaimiento del estado excitado [58].

Generalmente, los objetivos de la modificación superficial implican proporcionar cierta afinidad a las Si nps que permitan obtener suspensiones estables de nps, o generar posibilidades de reacción que la superficie original no proporciona, permitiendo así funcionalizaciones con moléculas de interés biológico. Existe gran cantidad de información sobre reacciones sobre superficies planas de Si [71]-[73], [74], que actualmente está en plena revisión para su aplicación en superficies curvas. 
Los métodos convencionales para funcionalizar superficies de Si involucran la formación de enlaces Si-C, cuya estabilidad es lo suficientemente alta para proteger la superficie de futuras oxidaciones. Existen diversas reacciones que llevan a la formación de estos enlaces, sin embargo la que ofrece la mayor ventaja es la reacción de hidrosililación [65], [74]-[77]. En ella, se adiciona Si y H sobre un enlace C-C insaturado. A su vez, los átomos de $\mathrm{Cl}$ o $\mathrm{Br}$ superficiales también permiten derivatizar la superficie de las nps mediante reacciones similares. Estas reacciones pueden ser llevadas adelante empleando alquenos terminales e iniciadas por luz [58] o calor [60], o empleando catalizadores de Pt [59]. La gran ventaja de estas reacciones es que no se rompen enlaces $\mathrm{Si}-\mathrm{Si}$, lo cual es de fundamental importancia para Si NP pequeñas. De hecho, las rutas que involucren el clivaje de dichos enlaces pueden ocasionar variaciones en las propiedades ópticas, y en el caso de partículas muy pequeñas hasta la disolución completa. Esta desventaja la presentan las reacciones de alcoxilación [54], donde pueden formarse enlaces Si-H o Si-O-C, y las alquilaciones por reactivos de Grignard o alquil-litios [62], [78], [79], donde la reacción procede por un ataque nucleofílico del carbanión sobre un átomo de Si con una baja densidad electrónica.

Sin embargo, diseñar y sintetizar Si nps con superficies modificadas por vía covalente con moléculas orgánicas o biomoléculas manteniendo sus propiedades fotofísicas es una tarea muy compleja. La unión directa de biomoléculas o cromóforos de interés puede llevar a pérdida de actividad o cambios en las propiedades, tanto de la molécula en superficie como de las Si nps. Debido a esto, suelen emplearse estrategias de múltiples pasos, que en general implican formar una plataforma reactiva sobre la superficie de las Si nps, para luego usar esta plataforma para la unión de moléculas de interés. En general, suelen usarse reacciones de silanización en las cuales se obtienen silanos con grupos terminales hidrolizables [62] o de hidrosililación con alquenos terminales que posean funciones amina o carboxilo [60], [80]. Sobre estas nuevas superficies, pueden unirse diversas moléculas usando distintas interacciones [38], [81].

El uso de Si nps en la obtención de imágenes y diagnóstico en sistemas biológicos está ligado a la conservación de sus propiedades luminiscentes, con lo cual es necesario contar con superficies estables en medios acuosos de concentraciones salinas y $\mathrm{pH}$ muy variables, y en presencia de proteínas diversas. En bibliografía se encuentra que el polietilenglicol (PEG) puede actuar como un agente de cobertura útil para tales fines. Hay diversos métodos que usan PEG para cubrir Si nps, desde la formación de micelas con fosfolípidos unidos a PEG [81], [82], hasta la unión directa sobre la superficie de las Si nps vía enlaces Si-O-C [83]. Finalmente, en la figura 1.1 resumimos los procesos más comunes de síntesis química y de modificación superficial. 


\section{Synthesis}

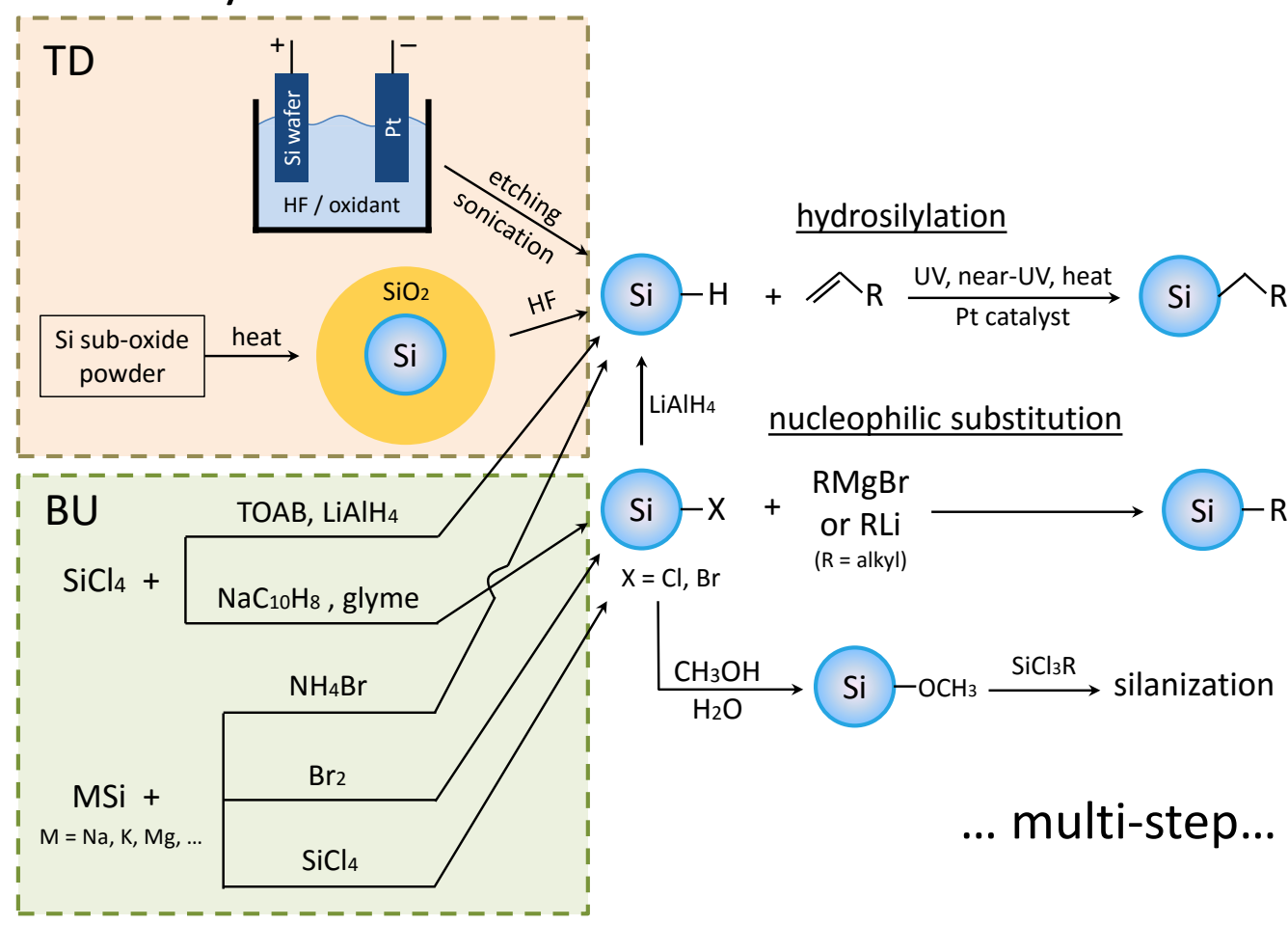

Figura 1.4. Vías de síntesis química y de modificación superficial típicas para Si nps [45].

\subsubsection{Propiedades fotofísicas}

En la actualidad, no existe una interpretación unívoca para las propiedades ópticas observadas en nanoestructuras de Silicio. Dentro de las Si nps, están muy bien documentadas dos bandas de emisión con propiedades muy diferentes: Por un lado, una banda en la región del rojo en espectro visible con tiempos de vida en los $\mu$ s, denominada banda S [7], [44], [84]-[87]; por el otro una banda en la región azul - verde con tiempos de vida en los ns, llamada banda F [58], [62], [63], [88]. En ambos casos el origen y propiedades de las dos bandas se atribuye a confinamiento cuántico [84][43], estados trampa sub - Band Gap [42] o defectos superficiales asociados a $\mathrm{N}$ y $\mathrm{O}$ [58], [89][90], lo cual muestra tanto la diversidad de fenómenos en competencia para el proceso de emisión como las múltiples posibilidades para la interpretación. Recientemente, varios grupos pioneros realizaron esfuerzos conjuntos para elucidar el problema, y observaron que la aparición de la banda $\mathrm{F}$ a partir de muestras que inicialmente solo presentan la banda $\mathrm{S}$ está relacionada con la carga de $\mathrm{N}$ y $O$ en su superficie [89], sin embargo la problemática dista mucho de estar resuelta. Actualmente, el trabajo se enfoca en elucidar la dinámica de los estados excitados involucrados al proceso de emisión, y las relaciones existentes entre estados que conducen a emisión en la banda $S$ y los que lo hacen a la banda $F$ [91].

\subsubsection{Funcionalización superficial y propiedades fotoluminiscentes}

Las propiedades superficiales juegan un rol clave en materiales nanoestructurados, desde los conocidos fenómenos termodinámicos y electrocinéticos [30], [92] hasta propiedades ópticas complejas [93]. Idealmente, estas propiedades podrían explotarse para aplicarlas en innovaciones tecnológicas. De todos modos, es necesario entender profundamente los procesos subyacentes que dan origen a estas nuevas propiedades a fin de construir dispositivos funcionales y robustos. A su vez, las propiedades luminscentes manifiestas en Si nps pueden ser excitadas usando distintas fuerzas impulsoras, dado lugar a quimioluminiscencia, electroluminiscencia o fotoluminiscencia [58], [94], [95]. 
Existen en bibliografía muchos estudios cuidadosos sobre el efecto de distintos ligandos y reacciones superficiales sobre las propiedades de luminiscencia, incluyendo halógenos vía halogenación [90], alquenos y alquinos vía hidrosililación [86], [96] o por reacciones de adición nucleofílica [59], [97], silanización u oxidaciones usando procesos suaves [15], [62]. Sin embargo, aunque haya gran cantidad de datos en bibliografía (tabla 1.1), la evidencia experimental es ciertamente divergente y hace difícil la propuesta de un modelo único. De todos modos, el conocimiento sobre el efecto de la superficie aumentó sustancialmente en los últimos años, logrando un consenso sobre los efectos debidos a ciertos arreglos superficiales cuyos cambios sobre las propiedades de PL son claramente determinados: Las coberturas del tipo Si-C (alquílicas o alquenílicas) sobre Si nps no afectan sustancialmente la densidad de estados en el Band-Gap comparado al caso de Si nps $\mathrm{H}$ - terminales, tal que las coberturas basadas en $\mathrm{C}$ pueden actuar efectivamente como coberturas sin efecto sobre las propiedades de emisión[77]. En el caso de sitios o enlaces polarizados (en presencia de $\mathrm{O}, \mathrm{N}$ u OX siendo $\mathrm{X}$ un halógeno), estos pueden actuar como trampas sub Band-Gap que dan lugar a emisión en tiempos cortos [58], [90]. También hay cierto acuerdo con la posibilidad de que interacciones fuertes entre la superficie de Si y ligandos con átomos de $\mathrm{N}$ puedan ser empleadas para sintonizar la posición de la banda de emisión sin cambiar el tamaño del agregado [91], [98].

\begin{tabular}{|c|c|c|c|c|c|}
\hline Si np & Solvente & $\begin{array}{l}\Phi \text { (atm. } \\
\text { inerte) }\end{array}$ & $\begin{array}{c}\Phi \text { (atm. } \\
\text { aire) }\end{array}$ & $\Phi_{\Delta}$ & Ref. \\
\hline $\mathrm{d}=3 \mathrm{~nm}, \mathrm{Si} / \mathrm{SiO}_{\mathrm{x}}$ core-shell, cubiertas & Tolueno & 0.76 & 0.43 & 0.15 & [42] \\
\hline con metacrilato de metilo & Agua & 0.20 & 0.18 & 0.03 & \\
\hline $\mathrm{d}=3 \mathrm{~nm} \mathrm{Si} / \mathrm{SiO}_{x}$ core-shell & Tolueno & 0.32 & 0.27 & & [42] \\
\hline $\begin{array}{c}\mathrm{d}=5 \mathrm{~nm}, \text { cubiertas con ligandos } \\
\text { orgánicos }\end{array}$ & & 0.60 & ---- & & [85] \\
\hline $\mathrm{d}=5 \mathrm{~nm}$ obtenidas por reducción & Agua & & 0.10 & & [58] \\
\hline química, cubiertas con propil amina & Agua & & 0.02 & & [81] \\
\hline $\mathrm{d}=3 \mathrm{~nm}$ obtenidas por oxidación & Acetonitrilo & & ---- & 0.12 & [83] \\
\hline química, cubiertas con PEG & Agua & & 0.14 & 0.03 & \\
\hline Silicio Poroso (PSi) & $\begin{array}{l}\text { Etanol } \\
\text { Agua }\end{array}$ & & & $\begin{array}{l}0.10 \\
0.17\end{array}$ & [7] \\
\hline $\mathrm{d}=5 \mathrm{~nm}$, síntesis vía plasma & DMF & 0.62 & & & [43] \\
\hline $\mathrm{d}=4 \mathrm{~nm}$ cubiertas con grupos octilos & $\begin{array}{l}\text { Hexano } \\
\text { Agua }\end{array}$ & & $\begin{array}{l}0.32 \\
0.18\end{array}$ & & [99] \\
\hline
\end{tabular}

Figura 1.4. Propiedades fotofísicas de Si nps, según reportado en bibliografía. $\Phi$ es el rendimiento cuántico de emisión, y $\Phi_{\Delta}$ es el rendimiento cuántico de generación de oxígeno singlete.

Los siguientes pasos hacia un modelo comprehensivo deberían incluir la obtención de evidencia espectroscópica y de experimentos ultrarrápidos sobre la dinámica de los estados involucrados en el proceso de emisión, tanto desde el lado experimental como teórico, para permitir el control de las propiedades de emisión variando el tamaño de las Si nps y el estado de su superficie. Por el momento, las aplicaciones tecnológicas de Si nps como fotosensibilizadores o fotocatalizadores serán guiadas por la evidencia experimental actualmente disponible, en los casos que sintonizar las bandas de absorción o emisión no sea una limitación.

\subsubsection{Potenciales Aplicaciones como Marcadores Biológicos}

La prevención y la detección de los tumores es un componente fundamental de todos los planes de lucha contra el cáncer ya que alrededor del $30 \%$ de las muertes por esta enfermedad se podrían prevenir [100]. Como consecuencia, grandes esfuerzos se destinan a obtener compuestos que puedan actuar con tal propósito y ser capaces de detectar tumores más efectivamente.

Con este fin, las nanopartículas semiconductoras de los grupos II-VI fueron centro de atención de investigación y en la actualidad [3] junto con los fluoróforos orgánicos (ej. rodaminas, cianinas y fluoresceínas) están muy 
desarrollados como biomarcadores a nivel de investigación [101]. El principal obstáculo para su implementación real, radica en que ambas familias de compuestos presentan importantes problemas y contraindicaciones asociadas a su naturaleza química. Los fluoróforos orgánicos tienen inconvenientes debido a su baja estabilidad, dificultad en la modulación de la longitud de onda de emisión y pequeño corrimiento de Stokes [80]. Como alternativa, aparecen las nanopartículas del grupo II-VI que muestran una mayor estabilidad y fácil modulación de la longitud de onda de emisión, sin embargo, muestran graves problemas centrados principalmente en la liberación de iones altamente nocivos (ej. $\mathrm{Cd}^{2+}$ ) cuando los recubrimientos no son estables en el entorno biológico [20]. Para minimizar los efectos de disolución de estos iones, las partículas son recubiertas por una capa adicional de ZnS, la cual aumenta su tamaño haciéndolas menos accesibles a la incorporación por parte de las células y dificultando su eliminación del organismo [101]. A pesar de este recubrimiento, no se garantiza la estabilidad de las propiedades ni un efectivo y completo aislamiento de los elementos tóxicos que liberan las nanopartículas al medio. A raíz de los significantes progresos en los estudios y aplicaciones de las nanopartículas del grupo II - VI se empezó a desarrollar el interés por las potenciales aplicaciones de las Si nps [102]. Estas partículas presentan importantes ventajas sobre las partículas del grupo II - VI, ya que muestran las propiedades fluorescentes debido al confinamiento cuántico de los elementos semiconductores y no contienen elementos que puedan presentar toxicidad. Por otra parte, debido a su pequeño tamaño (menor de $5 \mathrm{~nm}$ ), son capaces de penetrar las membranas celulares o los núcleos [66], [100] siendo este un requisito para su utilización como biomarcadores. La superficie de las nanopartículas de silicio es fácil de modificar con distintos agentes químicos que pueden variar y hacer posible su bioconjugación [74]. En la bibliografía se postula que las partículas recubiertas con polímeros son biocompatibles [82], [83], [85] y pueden conducir al desarrollo de detectores biocompatibles de pequeños tamaños [102].

\subsubsection{Potenciales Aplicaciones en Terapias Fotodinámicas}

Una de las terapias más empleadas en el tratamiento contra el cáncer tiene como eje central la utilización de radiación ionizante como los rayos $X$ y los rayos gamma [Hall 2006]. El principal inconveniente que presentan estas terapias se encuentra el daño producido a las células sanas circundantes al tumor, las cuales también son alcanzados por la radiación [Park 2006].

Como alternativa, se proponen las terapias fotodinámicas (PDT), en las cuales se utiliza la interacción entre la luz y un sensibilizador para iniciar la apoptosis de las células cancerígenas [103], [104]. En el enfoque moderno de PDT el agente sensibilizador se activa mediante luz IR, preferiblemente en el rango de longitudes de onda de 700 a $1100 \mathrm{~nm}$, donde la penetración es más profunda ya que la mayoría de cromóforos de los tejidos como la oxihemoglobina, desoxihemoglobina, melanina y grasa absorben débilmente [103]. El agente sensibilizador excitado no debería reacciona directamente con las células y los tejidos. Sin embargo, transfiere su estado de energía triplete a moléculas de oxígeno cercanas para generar oxígeno singlete, el cual es capaz de producir la muerte celular por distintos mecanismos (entre ellos la apoptosis) cuando son generadas en el interior de las células [105], [106]. Este tratamiento es altamente selectivo, solo los tejidos que se exponen simultáneamente al sensibilizador y a la luz, en presencia de oxígeno, presentan las reacciones citotóxicas. Por lo tanto, bajo circunstancias ideales en las que se consigue dirigir el fotosensibilizador a los tejidos enfermos, solo estos son erradicados, dejando los alrededores sanos sin daño celular. Hasta el momento, se han realizado estudios sobre el efecto de la utilización de las nanopartículas de los grupos VI-II [3], [101] como posibles sensibilizadores, ya que tienen la capacidad de generar especies reactivas de oxígeno cuando son llevadas a estados excitados mediante la exposición a luz de distintas longitudes de onda. Así mismo, nuevas modalidades de tratamientos basadas en la luminiscencia de las partículas que combina la radioterapia y la terapia fotodinámica ha sido estudiada con nanopartículas de CdSe recubiertas con diferentes sensibilizadores, siendo las nanopartículas excitadas mediante rayos $X$ transfiriendo su energía a los sensibilizadores, los cuales generan especies reactivas de oxígeno. 


\subsection{Transferencia de carga}

Las reacciones de transferencia de carga son ubicuas en la naturaleza, por ejemplo, el proceso de separación de cargas es uno de los pasos más importantes de la fotosíntesis, involucrado en la transformación de energía solar en potencial químico. Esta estrategia inspiró distintos enfoques que hoy en día se denominan fotosíntesis artificial [107]. Estos involucran el diseño y ensamblado de sistemas capaces de producir combustibles solares, celdas de combustible fotoelectroquímicas, e ingeniería de enzimas de organismos fotoautotróficos. A su vez, la nanotecnología contribuyó a este campo con nuevos materiales activos para conversión de energía y buscando el re-diseño de sistemas para aumentar su eficiencia [21]. Entender la fisicoquímica detrás de estas reacciones fue un proceso largo, que incluyó un trabajo mancomunado entre la electroquímica y la fotoquímica [108] y culminó con los trabajos de Marcus sentando las bases teóricas para comprender el proceso [109][111].

Las propiedades redox de las moléculas pueden ser mejoradas en el estado excitado. En estos casos, las reacciones proceden según las siguientes reacciones:

$$
\begin{aligned}
& { }^{1} D+A \rightarrow D^{++}+A^{-} \\
& { }^{1} A+D \rightarrow A^{-}+D^{+}
\end{aligned}
$$

Donores (D) típicos son la trimetilanilina, naftaleno, antraceno, perileno, fenantreno, etc; mientras que aceptores (A) ampliamente usados son las quinonas o el 9,10 - dicianoantraceno.

En reacciones en solución y en caso de que esta no sea controlada por difusión, puede determinarse la constante de transferencia de carga $k_{\mathrm{e}}$ [49], [112]. Hay 2 casos posibles:

- Si la interacción entre el donor y el aceptor en el par reactivo es fuerte, este par reactivo forma un exciplejo y se estudia como tal.

- Si la interacción entre el donor y el aceptor es débil, la reacción puede estudiarse usando la teoría de Marcus.

La teoría de Marcus predice una dependencia cuadrática de la energía libre de activación con la energía libre estándar de reacción:

$$
\Delta \mathrm{G} \pm=\left(\Delta \mathrm{G}^{0}+\lambda\right)^{2 / 4 \lambda}
$$

Siendo $\lambda$ la energía de reorganización, que está dada por la suma de contribuciones de esfera interna (debido a cambios estructurales de las especies donoras y aceptoras) y externa (reorganización del solvente) [113].

Teniendo en cuenta la relación de $k_{e}$ con $\Delta G \ddagger$, si se realiza un gráfico de ln $k_{e T}$ vs $\Delta G^{0}$ se observa una parábola con máximo en $\Delta \mathrm{G}^{0}=-\lambda$. Si $\Delta \mathrm{G}^{0}>-\lambda$, In $\mathrm{k}_{\mathrm{e}}$ aumenta cuando $-\Delta \mathrm{G}^{0}$ aumenta, que es llamada región normal de Marcus, ya que se comporta según lo observado para reacciones en general. Sin embargo, en el caso de $\Delta \mathrm{G}^{0}$ $<-\lambda$, In $k_{\text {et }}$ disminuye cuando $-\Delta G^{0}$ aumenta, lo cual constituyó un hecho completamente inesperado y se lo denominó región invertida de Marcus [114], [115].

\subsection{Plasmónica}

La Plasmónica es un área que combina la nanotecnología con la fotónica, que se basa en el estudio de los procesos de interacción entre la radiación electromagnética y los electrones de conducción en interfases metaldieléctrico. Los comportamientos que se observan como consecuencia de dicha interacción pueden interpretarse en base a la existencia de plasmones - oscilaciones colectivas de los electrones de conducción presentes en un metal tipo Drude - que poseen características relacionadas con el metal, su geometría y sus dimensiones, la longitud de onda de iluminación y el medio circundante [93]. 
Si bien sus bases se cimientan a principios del siglo XX con trabajos teóricos de A. Sommerfeld y observaciones experimentales de R. W. Wood, el avance en las técnicas de fabricación a escalas de $100 \mathrm{~nm}$, los métodos de caracterización de alta sensibilidad, el incremento sustancial en el poder de cálculo de las computadoras actuales y su amplio potencial de aplicaciones han motivado una expansión generalizada en el interés por este tema. La caracterización de estos plasmones puede utilizarse convenientemente para la determinación de tamaños de nanopartículas (Nps) de metales nobles menores que $10 \mathrm{~nm}$, tanto en estructuras esféricas simples como en estructuras más complejas tipo core-shell (metal-metal óxido o metal-dieléctrico), como también para el diseño de sensores plasmónicos de multicapas o de partícula aislada, de interés tanto en procesos industriales como biológicos [21][116][26].

Las oscilaciones plasmónicas en nanoestructuras metálicas permiten confinar el campo eléctrico en regiones particulares del espacio, lo cual tiene un efecto muy importante en las propiedades de estados excitados aledaños [117]. Este control puede ser combinado con la selectividad de la fotoquímica. Las reacciones fotoquímicas juegan un rol importante tanto en la naturaleza como a nivel tecnológico, formando parte desde el proceso de fotosíntesis hasta de técnicas de depuración de aguas y transducción de energía solar [21]. Estas reacciones involucran estados excitados que ofrecen vías de reacción alternativas, las cuales conducen a productos diferentes o incluso inaccesibles comparadas a sus contrapartes térmicas. Sin embargo, el control preciso de los estados involucrados no es una tarea fácil. En este aspecto, la excitación de nanoestructuras metálicas en el entorno del cromóforo de interés pueden generar campos eléctricos intensos y altamente localizados [93], logrando poblar en mayor medida estados excitados determinados que conduzcan a procesos fotoquímicos controlados. Esta estrategia ha sido utilizada exitosamente tanto en el aumento de señales Raman como fluorescentes[117].

\subsection{Objetivos}

A partir de lo expuesto previamente, decidimos que un aporte útil para el desarrollo de aplicaciones basadas en Si nps debíamos contemplar al menos 2 aspectos: Por un lado, un profundo entendimiento de la fotofísica básica de Si nps, prestando especial interés al efecto de la superficie; mientras que por el otro es necesario que estos sistemas basados en Si nps puedan absorber en la región visible del espectro electromagnético. Para lograr estas metas, en este trabajo focalizamos los experimentos primero en la comprensión básica de la fotoquímica de Si nps pequeñas $(1-5 \mathrm{~nm})$ que emiten en la región azul del espectro electromagnético, para luego preparar y estudiar sistemas que emplean estas Si nps como bloques de construcción y que muestran propiedades ópticas nuevas y con posibilidad de ser modulables, ya sea por procesos de transferencia de carga o por acoplamientos entre estados excitados y plasmónicos.

Como hipótesis de esta tesis se tiene: "Las Si nps poseen la capacidad de actuar como eficientes dadores y/o aceptores de carga/energía." Para su comprobación o rechazo se plantearon los siguientes objetivos:

- Sintetizar, caracterizar y evaluar la estabilidad en suspensión acuosa y en solventes orgánicos, de Si nps conjugados con diversos colorantes orgánicos y con nanopartículas de oro.

- Investigar la dinámica de los procesos físicos y químicos, en particular aquellos que involucran transferencia de carga o energía.

- Evaluar la eficiencia de la transferencia de energía o carga en función de la naturaleza del aceptor (dador), el número de aceptores (dadores) de energía unidos a las Si nps, la constante de decaimiento radiativo y no radiativo del dador de energía, tamaño de partículas y forma.

En una primera etapa se realizó la síntesis de las nanopartículas con las cuales se llevaron a cabo todos los estudios posteriores. Para este objetivo se evaluaron distintos métodos de síntesis considerando accesibilidad de equipos y adaptación a requerimientos específicos. Una vez obtenidas las nanopartículas se procedió a la modificación superficial con distintos agentes químicos, determinándose su adecuado recubrimiento y distribución de tamaños. Posteriormente se procedió a la caracterización de sus propiedades fotofísicas en suspensión en distintos solventes en función de los diferentes recubrimientos superficiales, a fin de comprender 
profundamente los procesos fotofísicos involucrados en la emisión de las Si nps. Posteriormente, se sintetizaron díadas en las cuales las Si nps actúen como donoras o aceptoras de carga o energía. Se emplearon tanto colorantes orgánicos como nanopartículas de oro. Finalmente, estas fueron caracterizadas usando una variedad de técnicas y se estudió en detalle su fotofísica. El objetivo final de este trabajo es el diseño de nuevos sistemas nanoestructurados basados en Silicio que presenten propiedades ópticas adecuadas para su uso como sensibilizadores activos en la región visible del espectro electromagnético mejoradas con respecto a los bloques de construcción utilizados.

\subsection{Estructura y contenidos}

Este informe se divide en tres partes. La primera, Métodos, está compuesta por tres capítulos. En el Capítulo 2 describimos los métodos experimentales y teóricos que utilizamos a lo largo del trabajo. El capítulo 3 se enfoca en los detalles experimentales de las diferentes rutas de síntesis para obtener Si nps, Au nps y la unión a los distintos colorantes orgánicos.

La segunda parte, Nanopartículas y Ensamblados Supramoleculares, consta de cuatro capítulos donde discutimos los resultados obtenidos relacionados con los diferentes bloques de construcción que forman los sistemas preparados en esta tesis y los sistemas en sí. El Capítulo 4 trata los aspectos fotofísicos relacionados con Si nps ultrapequeñas, de $1-2 \mathrm{~nm}$ de diámetro, obtenidas por diversas rutas sintéticas. Discutimos su caracterización y cómo las propiedades superficiales juegan un rol preponderante en la fotofísica de estas $\mathrm{Si}$ nps. El Capítulo 5 trata sobre la caracterización y fotofísica de Si nps de distintos tamaños obtenidas por oxidación de fases de Zintl, que usamos en los experimentos descriptos en los siguientes capítulos. Prestamos especial atención al papel que juegan el tamaño y la superficie al momento de controlar el destino de los estados excitados generados. En el capítulo 6 empleamos las Si nps descriptas en el capítulo 5 como aceptoras de carga en díadas que emplean colorantes orgánicos. Utilizamos distintos colorantes, a fin de excitar el sistema en distintas regiones del espectro electromagnético, para obtener sistemas que puedan emplearse en distintas condiciones de irradiación. Describimos la síntesis, caracterización y estudiamos la fotoquímica de las díadas usando una batería de técnicas espectroscópicas. En el capítulo 7 empleamos las mismas Si nps para preparar ensamblados coloidales con Au nps con propiedades de luminiscencia y sensibilización de oxígeno singlete mejoradas. Describimos la preparación, caracterización y fotoquímica de los ensamblados, prestando atención al cambio en la luminiscencia según la relación Au : Si de los ensamblados, usándola como variable de ajuste para las propiedades a sintonizar.

En la Parte final se presentan las conclusiones generales del trabajo y perspectivas (Capítulo 8).

Las Referencias Bibliográficas se presentan al final de cada capítulo en una lista enumerada por orden de aparición de las citas en el texto.

\subsection{Referencias}

[1] R. P. Feynman, "There 's Plenty of Room at the Bottom," vol. 1, no. i, pp. 60-66, 1959.

[2] N. Science and T. Council, "National Nanotechnology Initiative: Strategic Plan. National Science and Technology Council Subcommittee on Nanoscale Science, Engineering , and Technology Committee on Technology."

[3] P. Alivisatos, "Colloidal quantum dots. From scaling laws to biological applications," Pure Appl. Chem., vol. 72, no. 1-2, pp. 3-9, 2000.

[4] a. L. Rogach, Semiconductor nanocrystal quantum dots: synthesis, assembly, spectroscopy and applications. 2008.

[5] J. Turkevich, P. C. Stevenson, and J. Hillier, "A study of the nucleation and growth processes in the 
synthesis of colloidal gold," Discuss. Faraday Soc., vol. 11, no. c, pp. 55-75, 1951.

[6] Y. Qin, X. Ji, J. Jing, H. Liu, H. Wu, and W. Yang, "Size control over spherical silver nanoparticles by ascorbic acid reduction," Colloids Surfaces A Physicochem. Eng. Asp., vol. 372, no. 1-3, pp. 172-176, 2010.

[7] M. J. Sailor, Porous Silicon in Practice: Preparation, Characterization and Applications. 2012.

[8] D. S. Goodsell, "Lessons from Nature."

[9] J. Liqiang, Q. Yichun, W. Baiqi, and L. Shudan, "Review of photoluminescence performance of nanosized semiconductor materials and its relationships with photocatalytic activity," vol. 90, pp. 17731787, 2006.

[10] V. J. Mohanraj and Y. Chen, "Nanoparticles - A Review," vol. 5, no. June, pp. 561-573, 2006.

[11] P. K. Jain, X. Huang, and I. H. El-sayed, "Review of Some Interesting Surface Plasmon Resonanceenhanced Properties of Noble Metal Nanoparticles and Their Applications to Biosystems," pp. 107118, 2007.

[12] M. C. Daniel and D. Astruc, "Gold Nanoparticles: Assembly, Supramolecular Chemistry, QuantumSize-Related Properties, and Applications Toward Biology, Catalysis, and Nanotechnology," Chemical Reviews, vol. 104, no. 1. pp. 293-346, 2004.

[13] C. Burda, X. Chen, R. Narayanan, and M. A. El-Sayed, "Chemistry and properties of nanocrystals of different shapes," Chemical Reviews, vol. 105, no. 4. pp. 1025-1102, 2005.

[14] C. Vericat, M. E. Vela, G. Benitez, P. Carro, and R. C. Salvarezza, "Self-assembled monolayers of thiols and dithiols on gold: new challenges for a well-known system," Chem. Soc. Rev., vol. 39, no. 5, p. 1805, 2010.

[15] G. Allan, C. Delerue, and M. Lannoo, "Nature of Luminescent Surface States of Semiconductor Nanocrystallites," Phys. Rev. Lett., vol. 76, pp. 2961-2964, 1996.

[16] M. Idowu and T. Nyokong, "Interaction of water-soluble CdTe quantum dots with octacarboxy metallophthalocyanines: A photophysical and photochemical study," J. Lumin., vol. 129, pp. 356-362, 2009.

[17] S. Moeno and T. Nyokong, "The photophysical studies of a mixture of CdTe quantum dots and negatively charged zinc phthalocyanines," Polyhedron, vol. 27, pp. 1953-1958, 2008.

[18] S. D'Souza, E. Antunes, and T. Nyokong, "Synthesis and photophysical studies of CdTe quantum dotmonosubstituted zinc phthalocyanine conjugates," Inorganica Chim. Acta, vol. 367, no. 1, pp. 173181, 2011.

[19] M. Sanz, M. a. Correa-Duarte, L. M. Liz-Marzán, and A. Douhal, "Femtosecond dynamics of CdTe quantum dots in water," J. Photochem. Photobiol. A Chem., vol. 196, pp. 51-58, 2008.

[20] D. A. Tekdaş, M. Durmuş, H. Yanik, and V. Ahsen, "Photodynamic therapy potential of thiol-stabilized CdTe quantum dot-group 3A phthalocyanine conjugates (QD-PC)," Spectrochim. Acta - Part A Mol. Biomol. Spectrosc., vol. 93, pp. 313-320, 2012.

[21] P. V. Kamat, "Meeting the clean energy demand: Nanoestructure Architectures for Solar Energy Conversion," Phys. Chem., vol. 392, pp. 2834-2860, 2007. 
[22] Y. Yi, G. Zhu, C. Liu, Y. Huang, Y. Zhang, H. Li, J. Zhao, and S. Yao, "A label-free silicon quantum dots-based photoluminescence sensor for ultrasensitive detection of pesticides," Anal. Chem., vol. 85, no. 23, 2013.

[23] M. Ray, T. S. Basu, N. R. Bandyopadhyay, R. F. Klie, S. Ghosh, S. O. Raja, and A. K. Dasgupta, "Highly lattice-mismatched semiconductor-metal hybrid nanostructures: gold nanoparticle encapsulated luminescent silicon quantum dots," Nanoscale, vol. 6, pp. 2201-2210, 2014.

[24] J. Li and N. Wu, "Semiconductor-based photocatalysts and photoelectrochemical cells for solar fuel generation: a review," Catal. Sci. Technol., vol. 5, no. 3, pp. 1360-1384, 2015.

[25] I. Szilagyi, G. Trefalt, A. Tiraferri, P. Maroni, and M. Borkovec, "Polyelectrolyte adsorption, interparticle forces, and colloidal aggregation.," Soft Matter, vol. 10, pp. 2479-502, 2014.

[26] J. Gargiulo, S. Cerrota, E. Cortés, I. L. Violi, and F. D. Stefani, "Connecting Metallic Nanoparticles by Optical Printing," Nano Lett., vol. 16, no. 2, pp. 1224-1229, 2016.

[27] K. Ariga, J. P. Hill, and Q. Ji, "Layer-by-layer assembly as a versatile bottom-up nanofabrication technique for exploratory research and realistic application.," Phys. Chem. Chem. Phys., vol. 9, pp. 2319-2340, 2007.

[28] Y. Joseph, I. Besnard, M. Rosenberger, B. Guse, H.-G. Nothofer, J. M. Wessels, U. Wild, A. KnopGericke, D. Su, R. Schlögel, A. Yasuda, and Tobias Vossmeyer, "Self-Assembled Gold Nanoparticle/Alkanedithiol Films : Preparation, Electron Microscopy, XPS-Analysis, Charge Transport, and Vapor-Sensing Properties," J. Phys. Chem., vol. 107, pp. 7406-7413, 2003.

[29] Z. F. Li, M. T. Swihart, and E. Ruckenstein, "Luminescent silicon nanoparticles capped by conductive polyaniline through the self-assembly method," Langmuir, vol. 20, no. 19, pp. 1963-1971, 2004.

[30] J. N. Israelaschvili, "Intermolecular and surface forces." Academic Press Ltd.

[31] 'Self-Assembly in Natural and Unnatural Systems' Philp D., Fraser Stoddart, J. Angew. Chem. In. Ed. Engl. 1196, 35, 1154, 1196.".

[32] S. Zhang, D. M. Marini, W. Hwang, and S. Santoso, "Design of nanostructured biological materials through self-assembly of peptides and proteins," 2002.

[33] C. Graf and A. Van Blaaderen, "Metallodielectric colloidal core-shell particles for photonic applications," Langmuir, vol. 18, no. 19, pp. 524-534, 2002.

[34] C. Y. Liu and U. R. Kortshagen, A Silicon nanocrystal schottky junction solar cell produced from colloidal silicon nanocrystals, vol. 5, no. 8. 2010.

[35] J. D. Megiatto, A. Antoniuk-Pablant, B. D. Sherman, G. Kodis, M. Gervaldo, T. A. Moore, A. L. Moore, and D. Gust, "Mimicking the electron transfer chain in photosystem II with a molecular triad thermodynamically capable of water oxidation," Proc. Natl. Acad. Sci., vol. 109, no. 39, pp. 1557815583, 2012.

[36] Y. Terazono, G. Kodis, P. A. Liddell, V. Garg, T. A. Moore, A. L. Moore, and D. Gust, "Multiantenna artificial photosynthetic reaction center complex," J. Phys. Chem. B, vol. 113, no. 20, pp. 7147-7155, 2009.

[37] S. Moya and O. Azzaroni, "multilayers of poly ( diallyldimethylammonium and weak anionic groups $\dagger$," Phys. Chem. Chem. Phys., pp. 21-26, 2015. 
[38] E. Piccinini, D. Pallarola, and O. Azzaroni, "Molecular Systems Design \& Engineering materials by design," Mol. Syst. Des. Eng., 2016.

[39] B. T. Laboratories and M. Hill, "Formalisms of Band Theory."

[40] E. M. Pitaevskii, L., Lifshitz, "Curso de Física Teórica - Física Estadística II."

[41] E. M. Landau, L., Lifshitz, "Curso de Física Teórica - Física Estadística." .

[42] M. J. Llansola Portolés, P. M. David Gara, M. L. Kotler, S. Bertolotti, E. San Román, H. B. Rodríguez, and M. C. Gonzalez, "Silicon nanoparticle photophysics and singlet oxygen generation," Langmuir, vol. 26, no. 13, pp. 10953-10960, 2010.

[43] S. Yang, W. Li, B. Cao, H. Zeng, and W. Cai, "Origin of blue emission from silicon nanoparticles: Direct transition and interface recombination," J. Phys. Chem. C, vol. 115, pp. 21056-21062, 2011.

[44] V. Kuntermann, C. Cimpean, G. Brehm, G. Sauer, C. Kryschi, and H. Wiggers, "Femtosecond transient absorption spectroscopy of silanized silicon quantum dots," Phys. Rev. B, vol. 77, pp. 1-8, 2008.

[45] P. Caregnato, M. L. Dell'Arciprete, M. C. Gonzalez, C. R. Lillo, H. B. Rodríguez, and J. J. Romero, "Versatile silicon nanoparticles with potential uses as photoluminiscent sensors and photosensitizers," Photochemistry, vol. 44, pp. 322-345, 2017.

[46] G. Ledoux, O. Guillois, D. Porterat, C. Reynaud, F. Huisken, B. Kohn, and V. Paillard, "Photoluminescence properties of silicon nanocrystals as a function of their size," Phys. Rev. B, vol. 62, no. 23, pp. 15942-15951, 2000.

[47] M. Kuno and P. V Kamat, "Size-Dependent Electron Injection from Excited CdSe Quantum Dots into TiO 2 Nanoparticles," pp. 4136-4137, 2007.

[48] S. Dayal, R. Królicki, Y. Lou, X. Qiu, J. C. Berlin, M. E. Kenney, and C. Burda, "Femtosecond timeresolved energy transfer from CdSe nanoparticles to phthalocyanines," Appl. Phys. B Lasers Opt., vol. 84, pp. 309-315, 2006.

[49] J. R. Lakowicz, Principles of fluorescence spectroscopy. 2006.

[50] D. Bimberg, Semiconductor Nanostructure. 2008.

[51] J. Diener, M. Ben-Chorin, D. . Kovalev, S. . Ganichev, and F. Koch, "Excitation of the porous silicon photoluminescence by a multiphoton vibronic process," Thin Solid Films, vol. 276, pp. 116-119, 1996.

[52] D. Kovalev, H. Heckler, M. Ben-Chorin, G. Polisski, M. Schwartzkopff, and F. Koch, "Breakdown of the k-Conservation Rule in Si Nanocrystals," Phys. Rev. Lett., vol. 81, pp. 2803-2806, 1998.

[53] T. Orii, M. Hirasawa, T. Seto, N. Aya, and S. Onari, "Temperature dependence of photoluminescence from mono-dispersed Si nanoparticles," Eur. Phys. J. D, vol. 24, pp. 119-122, 2003.

[54] M. J. Llansola Portolés, F. R. Nieto, D. B. Soria, J. I. Amalvy, P. J. Peruzzo, D. O. Mártire, M. Kotler, O. Holub, and M. C. Gonzalez, "Photophysical properties of blue-emitting silicon nanoparticles," J. Phys. Chem. C, vol. 113, pp. 13694-13702, 2009.

[55] J. J. Romero, M. J. Llansola-Portolés, M. L. Dell'Arciprete, H. B. Rodríguez, A. L. Moore, and M. C. Gonzalez, "Photoluminescent 1-2 nm sized silicon nanoparticles: A surface-dependent system," Chem. Mater., vol. 25, pp. 3488-3498, 2013. 
[56] V. LaMer and R. Dinegar, "Theory, production and mechanism of formation of monodispersed hydrosols," J. Am. Chem. ..., vol. 72, no. 8, pp. 4847-4854, 1950.

[57] S. Yamanaka, "Silicon clathrates and carbon analogs: high pressure synthesis, structure, and superconductivity.," Dalton Trans., vol. 39, pp. 1901-1915, 2010.

[58] J. J. Romero, M. J. Llansola-portole, D. Arciprete, B. Rodr, A. L. Moore, and C. Gonzalez, "Photoluminescent 1 - 2 nm Sized Silicon Nanoparticles: A Surface- Dependent System," Chem. Mater., 2013.

[59] M. Rosso-Vasic, E. Spruijt, and B. Van Lagen, Alkyl-Functionalized Oxide-Free Silicon Nanoparticles: Synthesis and Optical Properties Cited by me. 2008.

[60] M. Rosso-vasic, L. De Cola, and H. Zuilhof, "Efficient Energy Transfer between Silicon Nanoparticles and a Ru - Polypyridine Complex," vol. 2, pp. 2235-2240, 2009.

[61] A. Shiohara, S. Hanada, S. Prabakar, K. Fujioka, T. H. Lim, K. Yamamoto, P. T. Northcote, and R. D. Tilley, "Chemical Reactions on Surface Molecules Attached to Silicon Quantum Dots," J. Am. Chem. Soc., vol. 132, no. 24, pp. 248-253, 2009.

[62] J. Zou, R. K. Baldwin, K. a. Pettigrew, and S. M. Kauzlarich, "Solution synthesis of ultrastable luminescent siloxane-coated silicon nanoparticles," Nano Lett., vol. 4, pp. 1181-1186, 2004.

[63] D. Neiner, H. W. Chiu, and S. M. Kauzlarich, "Low-temperature solution route to macroscopic amounts of hydrogen terminated silicon nanoparticles," J. Am. Chem. Soc., vol. 128, pp. 11016-11017, 2006.

[64] R. K. Baldwin, K. a. Pettigrew, J. C. Garno, P. P. Power, G. Y. Liu, and S. M. Kauzlarich, "Room temperature solution synthesis of alkyl-capped tetrahedral shaped silicon nanocrystals," J. Am. Chem. Soc., vol. 124, pp. 1150-1151, 2002.

[65] T. M. Atkins, a. Thibert, D. S. Larsen, S. Dey, N. D. Browning, and S. M. Kauzlarich, "Femtosecond Ligand/Core Dynamics of Microwave Assisted Synthesized Silicon Quantum Dots in Aqueous Solution.," J. Am. Chem. Soc., pp. 1-9, 2011.

[66] X. Zhang, D. Neiner, S. Wang, A. Y. Louie, and S. M. Kauzlarich, "A new solution route to hydrogenterminated silicon nanoparticles: synthesis, functionalization and water stability," vol. 95601, 2007.

[67] X. Ma, F. Xu, T. M. Atkins, A. M. Goforth, D. Neiner, A. Navrotsky, and S. M. Kauzlarich, "A versatile low temperature synthetic route to Zintl phase precursors: Na4Si4, Na4Ge4 and K4Ge4 as examples.," Dalton Trans., pp. 10250-10255, 2009.

[68] J. M. Chem, T. Yi, S. Chen, S. Li, H. Yang, S. Bux, Z. Bian, N. A. Katcho, A. Shakouri, N. Mingo, J. Fleurial, D. Browning, and S. M. Kauzlarich, "Synthesis and characterization of Mg $2 \mathrm{Si} / \mathrm{Si}$ nanocomposites prepared from $\mathrm{MgH} 2$ and silicon , and their thermoelectric properties †," pp. 2480524813, 2012.

[69] J. J. Romero, M. Wegmann, C. Lillo, A. Rubert, S. Klein, and C. Kryschi, "Impact of Iron Incorporation on 2 - 4 nm Size Silicon Nanoparticles 2 Properties 1," 2015.

[70] X. Zhang, M. Brynda, R. D. Britt, E. C. Carroll, D. S. Larsen, A. Y. Louie, and S. M. Kauzlarich, "Synthesis and Characterization of Manganese-Doped Silicon Nanoparticles: Bifunctional Paramagnetic-Optical Nanomaterial," pp. 10668-10669, 2007.

[71] G. T. Wang, C. Mui, J. F. Tannaci, M. a Filler, C. B. Musgrave, and S. F. Bent, "Reactions of cyclic 
aliphatic and aromatic amines on $\mathrm{Ge}(100)-2 \times 1$ and Si(100)-2x1," J. Phys. Chem. B, vol. 107, no. 100, pp. 4982-4996, 2003.

[72] M. D. Ellison and R. J. Hamers, "Adsorption of phenyl isothiocyanate on Si(001): A 1,2-dipolar surface addition reaction," J. Phys. Chem. B, vol. 103, no. 1, pp. 6243-6251, 1999.

[73] R. J. Hamers, S. K. Coulter, M. D. Ellison, J. S. Hovis, D. F. Padowitz, M. P. Schwartz, C. M. Greenlief, and J. N. Russell Jr., "Cycloaddition Chemistry of Organic Molecules with Semiconductor Surfaces.," Acc. Chem. Res., vol. 33, no. 9, pp. 617-624, 2000.

[74] J. M. Buriak, "Illuminating silicon surface hydrosilylation: An unexpected plurality of mechanisms," Chem. Mater., vol. 26, no. 1, pp. 763-772, 2014.

[75] J. a. Kelly and J. G. C. Veinot, "An investigation into near-UV hydrosilylation of freestanding silicon nanocrystals," ACS Nano, vol. 4, no. 8, pp. 4645-4656, 2010.

[76] F. Hua, F. Erogbogbo, M. T. Swihart, and E. Ruckenstein, "Organically capped silicon nanoparticles with blue photoluminescence prepared by hydrosilylation followed by oxidation," Langmuir, vol. 22, no. 20, pp. 4363-4370, 2006.

[77] F. Hua, M. T. Swihart, and E. Ruckenstein, "Efficient surface grafting of luminescent silicon quantum dots by photoinitiated hydrosilylation," Langmuir, vol. 21, no. 18, pp. 6054-6062, 2005.

[78] X. Cheng, S. B. Lowe, P. J. Reece, and J. J. Gooding, "Colloidal silicon quantum dots: from preparation to the modification of self-assembled monolayers (SAMs) for bio-applications," Chem. Soc. Rev., vol. 43, no. 8, p. 2680, 2014.

[79] D. S. English, L. E. Pell, Z. Yu, P. F. Barbara, and B. a. Korgel, "Size Tunable Visible Luminescence from Individual Organic Monolayer Stabilized Silicon Nanocrystal Quantum Dots," Nano Lett., vol. 2, pp. 681-685, 2002.

[80] E. V. Rogozhina, D. a. Eckhoff, E. Gratton, and P. V. Braun, "Carboxyl functionalization of ultrasmall luminescent silicon nanoparticles through thermal hydrosilylation," J. Mater. Chem., vol. 16, p. 1421, 2006.

[81] C. R. Lillo, J. J. Romero, M. L. Portolés, R. P. Diez, and P. C. Caregnato, "Organic-coating of 1-2 nm size silicon nanoparticles : effect on the particle properties," 2015.

[82] A. Papra, N. Gadegaard, and N. B. Larsen, "Characterization of ultrathin poly(ethylene glycol) monolayers on silicon substrates," Langmuir, vol. 17, no. 14, pp. 1457-1460, 2001.

[83] M. F. L. de M. and M. C. G. Damián Rodríguez Sartori, Cristian R Lillo, Juan J Romero, María Laura Dell'Arciprete, Alejandro Miñán, "Polyethylene glycol-coated blue-emitting silicon dots with improved properties for uses in aqueous and biological environments," Nanotechnology, vol. 27, no. 47, pp. 111.

[84] E. J. Henderson, J. a. Kelly, and J. G. C. Veinot, "Influence of HSiO1.5 sol-gel polymer structure and composition on the size and luminescent properties of silicon nanocrystals," Chem. Mater., vol. 21, no. 10, pp. 5426-5434, 2009.

[85] J. G. C. Veinot, "Synthesis, surface functionalization, and properties of freestanding silicon nanocrystals.," Chem. Commun. (Camb)., pp. 4160-4168, 2006.

[86] V. Groenewegen, V. Kuntermann, D. Haarer, M. Kunz, and C. Kryschi, "Excited-state relaxation 
dynamics of 3-vinylthiophene-terminated silicon quantum dots," J. Phys. Chem. C, vol. 114, pp. 11693-11698, 2010.

[87] C. Cimpean, V. Groenewegen, V. Kuntermann, A. Sommer, and C. Kryschi, "Ultrafast exciton relaxation dynamics in silicon quantum dots," Laser Photonics Rev., vol. 3, no. 1, pp. 138-145, 2009.

[88] J. H. Warner, H. Rubinsztein-Dunlop, and R. D. Tilley, "Surface morphology dependent photoluminescence from colloidal silicon nanocrystals," J. Phys. Chem. B, vol. 109, pp. 19064-19067, 2005.

[89] M. Dasog, Z. Yang, S. Regli, T. M. Atkins, A. Faramus, M. P. Singh, E. Muthuswamy, S. M. Kauzlarich, R. D. Tilley, and J. G. C. Veinot, "Chemical insight into the origin of red and blue photoluminescence arising from freestanding silicon nanocrystals," ACS Nano, vol. 7, no. 3, pp. 26762685, 2013.

[90] M. Dasog, K. Bader, and J. G. C. Veinot, "Influence of Halides on the Optical Properties of Silicon Quantum Dots," Chem. Mater., vol. 27, pp. 1153-1156, 2015.

[91] J. Fuzell, A. Thibert, T. M. Atkins, M. Dasog, E. Busby, J. G. C. Veinot, S. M. Kauzlarich, and D. S. Larsen, "Red states versus blue states in colloidal silicon nanocrystals: Exciton sequestration into lowdensity traps," J. Phys. Chem. Lett., vol. 4, pp. 3806-3812, 2013.

[92] R. Hiemenz, P.C., Rajagopalan, "Principles of Colloid and Surface Chemistry." MArcel DEkker, inc.

[93] K. L. Kelly, E. Coronado, L. L. Zhao, and G. C. Schatz, "Feature article," J. Phys. Chem. B, vol. 107, pp. 668-677, 2003.

[94] Z. Ding, B. M. Quinn, S. K. Haram, L. E. Pell, B. a Korgel, and A. J. Bard, "Electrochemistry and electrogenerated chemiluminescence from silicon nanocrystal quantum dots.," Science, vol. 296, no. 5571, pp. 1293-1297, 2002.

[95] A. J. Bard, L. R. Faulkner, N. York, C. @bullet, W. Brisbane, and S. E. Toronto, ELECTROCHEMICAL METHODS Fundamentals and Applications. 1944.

[96] R. J. Clark, M. K. M. Dang, and J. G. C. Veinot, "Exploration of organic acid chain length on watersoluble silicon quantum dot surfaces," Langmuir, vol. 26, no. 19, pp. 15657-15664, 2010.

[97] K. Pettigrew and Q. Liu, "Solution synthesis of alkyl-and alkyl/alkoxy-capped silicon nanoparticles via oxidation of Mg2Si," Chem. ..., no. 31, pp. 4005-4011, 2003.

[98] M. Dasog, Z. Yang, S. Regli, T. M. Atkins, A. Faramus, M. P. Singh, E. Muthuswamy, S. M. Kauzlarich, R. D. Tilley, and J. G. C. Veinot, "Chemical insight into the origin of red and blue photoluminescence arising from freestanding silicon nanocrystals," ACS Nano, vol. 7, pp. 2676-2685, 2013.

[99] Y. Kanemitsu, S. Okamoto, M. Otobe, and S. Oda, "Photoluminescence mechanism in surfaceoxidized silicon nanocrystals," Phys. Rev. B, vol. 55, no. 12, pp. R7375-R7378, 1997.

[100] S. Klein, M. L. Dell'Arciprete, M. Wegmann, L. V. R. Distel, W. Neuhuber, M. C. Gonzalez, and C. Kryschi, "Oxidized silicon nanoparticles for radiosensitization of cancer and tissue cells," Biochem. Biophys. Res. Commun., vol. 434, pp. 217-222, 2013.

[101] M. Baker, "Nanotechnology imaging probes: smaller and more stable," Nat. Methods, vol. 7, no. 12, pp. 957-962, 2010. 
[102] Y. He, Y. Su, X. Yang, Z. Kang, T. Xu, R. Zhang, C. Fan, and S. T. Lee, "Photo and pH stable, highlyluminescent silicon nanospheres and their bioconjugates for immunofluorescent cell imaging," J. Am. Chem. Soc., vol. 131, no. 12, pp. 4434-4438, 2009.

[103] H. Yanik, D. Aydin, M. Durmuş, and V. Ahsen, "Peripheral and non-peripheral tetrasubstituted aluminium, gallium and indium phthalocyanines: Synthesis, photophysics and photochemistry," J. Photochem. Photobiol. A Chem., vol. 206, pp. 18-26, 2009.

[104] Zhao, "Singlet Oxygen." p. 10, 2001.

[105] R. Scurlock and P. Ogilby, "Singlet molecular oxygen (1. DELTA. gO2) formation upon irradiation of an oxygen (3. SIGMA. g-O2)-organic molecule charge-transfer absorption band," J. Phys. Chem., no. 23, pp. 5493-5500, 1989.

[106] P. Ogilby and C. Foote, "Chemistry of singlet oxygen. 42. Effect of solvent, solvent isotopic substitution, and temperature on the lifetime of singlet molecular oxygen (1. DELTA. g)," J. Am. Chem. Soc., vol. 103, pp. 3423-3430, 1981.

[107] D. Gust, T. A. Moore, and A. L. Moore, "Mimicking photosynthetic solar energy transduction," Acc. Chem. Res., vol. 34, no. 1, pp. 40-48, 2001.

[108] S. Farid, J. P. Dinnocenzo, P. B. Merkel, R. H. Young, D. Shukla, and G. Guirado, "Reexamination of the Rehm À Weller Data Set Reveals Electron Transfer Quenching That Follows a Sandros À Boltzmann Dependence on Free Energy," vol. 0, no. Figure 1, pp. 11580-11587, 2011.

[109] R. A. Marcus, "Electrostatic Free Energy and Other Properties of States Having N onequilibrium Polarization. 1*," vol. 24, no. 5, pp. 10-13, 1956.

[110] R. A. Marcus, "ON T H E T H E O R Y O F ELECTROCHEMICAL AND CHEMICAL ELECTRON," vol. 37, no. 2, 1968.

[111] R. A. Marcus, "On the Theory of Oxidation-Reduction Reactions Involving Electron Transfer. 1*," vol. 42, no. 1952, pp. 966-978, 1956.

[112] B. Valeur, Molecular Fluorescence: Principles and applications, vol. 8. 2001.

[113] K. Ohkubo, H. Imahori, J. Shao, Z. Ou, K. M. Kadish, Y. Chen, G. Zheng, R. K. Pandey, M. Fujitsuka, and O. Ito, "Small Reorganization Energy of Intramolecular Electron Transfer in Fullerene-Based Dyads with Short Linkage," pp. 10991-10998, 2002.

[114] I. R. Could, J. E. Maser, and S. Farid, "Efficiencies of Photoinduced Electron-Transfer Reactions: Role of the Marcus Inverted Region in Return Electron Transfer within Geminate Radical-Ion Pairs," no. d, pp. 4290-4301, 1990.

[115] M. M. Waskasi, G. Kodis, A. L. Moore, T. A. Moore, and D. V Matyushov, "Marcus Bell-Shaped Electron Transfer Kinetics Observed in an Arrhenius Plot," no. 2, 2016.

[116] F. Balzarotti, Y. Eilers, K. C. Gwosch, A. H. Gynnå, V. Westphal, F. D. Stefani, J. Elf, and S. W. Hell, "Nanometer resolution imaging and tracking of fluorescent molecules with minimal photon fluxes," vol. 9913, no. December, 2016.

[117] K. Aslan, J. R. Lakowicz, and C. D. Geddes, "Metal-enhanced fluorescence using anisotropic silver nanostructures: Critical progress to date," Anal. Bioanal. Chem., vol. 382, pp. 926-933, 2005. 
- 35 - 
Parte I

Métodos 
- 37 - 


\section{Capítulo 2. Métodos Experimentales}

En este capítulo se presentarán brevemente las diferentes técnicas experimentales que hemos utilizado en este trabajo. Discutiremos con un grado mayor de detalle las técnicas y herramientas de cálculo más relevantes para esta tesis y en las que se trabajó activamente. En el caso de las técnicas de uso común o las que fueron llevadas a cabo mediante el trabajo en colaboración sólo enumeraremos las condiciones particulares de medida de los experimentos realizados y discutidos en esta tesis.

\subsection{Espectroscopía estacionaria de absorción UV-Visible}

La absorción de radiación electromagnética $(\mathrm{RE})$ es el proceso en el cual la materia toma energía por interacción con un fotón. Este fenómeno puede ser entendido a partir de variables macroscópicas por medio de la ley de Lambert-Beer, cuyas posibles expresiones se condensan en la ecuación (2.1):

$$
\begin{aligned}
& A\left(\lambda_{\text {exc }}\right)=-\log T\left(\lambda_{\text {exc }}\right)=-\log \left[\frac{I_{T}\left(\lambda_{\text {exc }}\right)}{I_{0}\left(\lambda_{\text {exc }}\right)}\right]=\varepsilon\left(\lambda_{\text {exc }}\right) / C \\
& I_{A}\left(\lambda_{\text {exc }}\right)=I_{0}\left(\lambda_{\text {exc }}\right)-I_{T}\left(\lambda_{\text {exc }}\right)=I_{0}\left(\lambda_{\text {exc }}\right)\left(1-10^{-A\left(\lambda_{\text {exc }}\right)}\right)
\end{aligned}
$$

Donde A es la absorbancia de la muestra, que se relaciona de forma logarítmica con la transmitancia $\mathrm{T}$. Esta se define como el cociente entre la intensidad transmitida por la muestra $\left(I_{T}\right)$ y la intensidad incidente $\left(I_{0}\right)$. A su vez, la absorbancia puede expresarse como el producto entre el camino óptico (I), el coeficiente de absorción molar $(\varepsilon)$ y la concentración molar $(C)$ de la especie absorbente. Puede verse en (2.1) que las variables dependen de la longitud de onda de excitación $\lambda_{\text {exc. }}$ Las ecuaciones (2.1) y (2.2) se emplean posteriormente en el cálculo de rendimientos cuánticos de emisión por el método del patrón².

Cuando se trabaja con material en suspensión los espectros de absorción presentan una desviación debido a la dispersión de RE incidente que no alcanza al detector y por tanto es interpretada como una absorción [1]. Dependiendo de la interacción entre el fotón y el analito, se producirá dispersión Raman o Rayleigh. En los casos donde las partículas son de un tamaño menor a un 10\% de la longitud de onda incidente predomina el efecto Rayleigh.

La luz dispersada a partir de la luz incidente es detectada como absorción, pero no necesariamente excita la muestra y no genera procesos de interés fotoquímico que puedan ser analizados. Por lo tanto, el espectro obtenido directamente del equipo es de atenuación y hay que eliminar la cantidad de luz dispersada por las nanopartículas, ya sea mediante el empleo de una esfera integradora o mediante una estimación de la luz dispersada, para obtener el espectro de absorción.

$$
\begin{aligned}
& \frac{I_{T}\left(\lambda_{\text {exc }}\right)}{I_{0}\left(\lambda_{\text {exc }}\right)}=a+b\left(\frac{1}{\lambda}\right)^{4} \\
& \frac{I_{T}\left(\lambda_{\text {exc }}\right)}{I_{0}\left(\lambda_{\text {exc }}\right)}=a^{\prime}+b^{\prime}\left(\frac{1}{\lambda}\right)^{c}
\end{aligned}
$$

\footnotetext{
${ }^{2}$ Este método se detalla en la sección 2.X. Espectrometría de emisión del presente capítulo.
} 
Trabajando a concentración constante y bajo las mismas condiciones de adquisición, se puede emplear la ecuación 2.3 , en la que se relaciona la cantidad de luz dispersada con la inversa de la longitud de onda elevada a la cuarta potencia, relación obtenida por Rayleigh originalmente [2]. Cuando el ajuste a la ecuación (2.3) en regiones alejadas de las bandas de absorción es pobre, los espectros se ajustan a la ecuación (2.4), la cual tiene en cuenta la agregación de las partículas con un parámetro extra c que varía desde 4 para sistemas totalmente desagregados e ideales hasta valores de $-2,2$ para sistemas de distribuciones y agregación muy irregulares [3].

Las medidas de atenuación de la RE se realizaron con un espectrofotómetro de doble haz Shimadzu UV-1800, con ancho de banda entre 0,2 y 4,0 nm según el ancho de rendija seleccionado y velocidad de barrido de 300 $\mathrm{nm} / \mathrm{min}$, o con espectrofotómetro de doble haz T90 + UV/Vis (PG Instruments Ltd.), con las mismas condiciones de adquisición. Tanto en estas medidas como en las de emisión, las muestras se emplazaron en celdas de cuarzo o de vidrio según lo requiera el rango espectral, de $10.00 \mathrm{~mm}$ de camino óptico. Previo a cada uso, estas fueron lavadas con agua regia ${ }^{3}$, para remover materia orgánica o metales. Para la generación de atmósferas especiales en las medidas experimentales, las muestras se burbujean durante 15 minutos empleando $\mathrm{Ar} \mathrm{u} \mathrm{O}_{2}$ a fin de saturar las dispersiones, o se las deja abiertas a la atmósfera para lograr un equilibrado con aire.

\subsection{Espectroscopía estacionaria de absorción en el IR}

La Espectroscopia de Infrarrojo es una técnica espectroscópica versátil y de gran aplicación en la caracterización de materiales, ya que permite la identificación de los grupos funcionales de un compuesto. El espectrómetro de IR con transformada de Fourier permite la obtención de espectros de forma rápida, precisa y con relaciones Señal/Ruido (S/N) elevadas. La técnica de ATR (del inglés Attenuated Total Reflection) es una técnica de muestreo utilizada en el IR que se basa en la reflexión que experimenta la RE en la interfase de separación de dos medios de distinto índice de refracción (n) cuando esta viene del medio más denso. Se producen dos fenómenos: la refracción y la reflexión, observándose más este segundo cuanto mayor es el ángulo de incidencia. Superado cierto valor de dicho ángulo (ángulo crítico, que es función del $\eta$ de ambos medios), se produce la reflexión total de la radiación. En espectroscopia ATR se coloca en estrecho contacto con la muestra un cristal de alto índice de refracción y buenas propiedades de transmisión de la radiación IR. De este modo, cuando se hace pasar radiación IR por este cristal con un ángulo de incidencia igual a superior al crítico, la radiación experimentará reflexión total en la interfase muestra-cristal. De esta forma se genera una onda evanescente que penetra la muestra entre 2 y $15 \mu \mathrm{m}$. La intensidad de esta onda evanescente decae con la distancia a la interfase, por lo cual para observar el espectro IR de la muestra es esencial que exista un contacto real de la muestra y el cristal. Normalmente, los espectros ATR son similares a los espectros IR convencionales excepto en las regiones de longitudes de onda más cortas porque, en general, estas RE penetran menos en la muestra y por tanto absorben menos. Cabe aclarar que el grado de penetración no sigue una dependencia estrictamente proporcional de la longitud de onda porque, si bien por una parte depende directamente de esta, existe una dependencia con los índices de refracción de la muestra y el cristal, que a su vez dependen de la longitud de onda de la RE.

Los espectros infrarrojos con transformada de Fourier (FTIR) se obtuvieron con un equipo Bruker EQUINOX 25 . Se registraron los espectros entre 4000 y $400 \mathrm{~cm}^{-1}$ con resolución de $1 \mathrm{~cm}^{-1}$. Se tomaron promedios de 64 medidas con una resolución de $4 \mathrm{~cm}^{-1}$. Se utilizaron tanto pastillas de $\mathrm{KBr}$ como cristales de silicio $<100>$ como soporte de las muestras, las que se sembraron sobre la superficie. Los espectros de reflexión total infrarroja atenuada (ATR-FTIR) se obtuvieron mediante un equipo Nicolet 380 spectrometer usando un cristal de ZnSe con un ángulo de incidencia del haz de $45^{\circ}$.

La preparación de pastillas de $\mathrm{KBr}$ se realiza prensando con una presión de 3 toneladas $/ \mathrm{cm}^{2}$ una mezcla de $\mathrm{KBr}$ con la muestra sólida (en caso de ser nps, se secaron previamente en estufa a $40^{\circ} \mathrm{C}$ y $0,2 \mathrm{~atm}$ ). La ventaja de utilizar la pastilla de $\mathrm{KBr}$ como soporte radica en su sencilla y rápida preparación. Además, al tener gran

\footnotetext{
${ }^{3}$ La preparación y uso del agua regia se explica en el capítulo 3.
} 
cantidad de muestra los espectros se obtienen bien definidos y con una buena calidad señal/ruido. Cuando las medidas se encuentran condicionadas por la poca cantidad de la que se dispone se utilizó un cristal de silicio como soporte ya que este es transparente en la ventana de número de onda a las que se observa el espectro infrarrojo. Para ello se realiza una siembra de la suspensión de partículas gota a gota y constantemente se evapora el solvente en atmosfera inerte de Ar a temperatura ambiente. Se añaden gotas de la muestra hasta la obtención de espectros con una relación señal/ruido adecuada. En estos casos se utilizó como blanco una oblea de cristal de silicio sin sembrar.

\subsection{Voltamperometría cíclica}

En la voltamperometría cíclica (CV, del inglés Cyclic Voltammetry) se utiliza una celda de tres electrodos: el electrodo de trabajo, el electrodo de referencia y el electrodo auxiliar (o contraelectrodo). Estos electrodos están conectados a los terminales de un potenciostato. Los potenciostatos utilizados para realizar experiencias de CV permiten aplicar una rampa de potencial (generalmente una onda triangular) entre el electrodo de trabajo y el electrodo de referencia. Es decir, controla el potencial del electrodo de trabajo referido al de referencia, utilizando una impedancia muy grande de manera tal que no circule corriente por el electrodo de referencia. La función que se aplica tiene la siguiente forma:

$$
E(t)=E_{i}+v t
$$

donde $E(t)$ es el potencial en el instante de tiempo $t, E_{i}$ es el potencial inicial y v es la velocidad de barrido del potencial. El barrido lineal se genera entre diferentes límites, los que se designan aquí como límite mayor de potencial, $E_{\max }$ y límite menor, $E_{\min }$. Los valores típicos de $\mathrm{v}$ van de $10^{-3}$ a $1 \mathrm{Vs}^{-1}$. A medida que se modifica el potencial, se registra la corriente que circula entre el electrodo de trabajo y el auxiliar. Con los datos registrados por el potenciostato se crea un gráfico de intensidad i o densidad de corriente $j(j=i / A)$ en función del potencial E denominado voltamperograma, donde A es el área real del electrodo En estos gráficos se observan diferentes procesos como los de transferencia de carga con especies electroactivas y los de carga de la denominada doble capa eléctrica. Un análisis detallado de esta técnica puede encontrarse en el texto de Bard y Faulkner [4]

Las medidas electroquímicas se realizaron utilizando un potenciostato $760 \mathrm{D}$ de $\mathrm{CH}$ Instruments controlado desde PC, en material de vidrio cuidadosamente lavado (Con potasa alcohólica, luego solución piraña y finalmente agua ultrapura). Como electrodo de referencia se usó un electrodo de calomel saturado mientras que como contraelectrodo un disco de platino. Los electrodos de trabajo se prepararon depositando las Si nps sobre vidrios de óxido de estaño dopado con flúor (FTO). A tal fin, se agregó gota a gota una suspensión acuosa de las Si nps a estudiar dejando evaporar el solvente en corriente de nitrógeno. Una vez logrado un depósito de aspecto claro, se sumergió el electrodo en una solución de 1,10-bis(trietoxisilil)decano $3 \mathrm{mM}$ en ciclohexano durante $20 \mathrm{hs}$, a fin de crear un film entrecurzado sobre el depósito y hacerlo más robusto, para evitar la disolución del depósito en el electrolito soporte. Esta técnica para obtener depósitos estables está documentada en bibliografía y permite el estudio del analito depositado sin interferencia del agente de entrecruzado [5]. Estos electrodos se dejaron secar a temperatura ambiente para finalmente ser usados.

\subsection{Espectroscopía fotoelectrónica de rayos X (XPS)}

XPS es una técnica semi-cuantitativa y de baja resolución espacial que habitualmente se utiliza para estimar la estequiometría, estado químico y estructura electrónica de los elementos que existen en la superficie de un material (de 1 a $10 \mathrm{~nm}$ de profundidad). Los espectros XPS son obtenidos cuando se irradia una muestra con rayos $X$, al tiempo que se mide la energía cinética y el número de electrones que escapan de la superficie a analizar. Para una medida de XPS se requieren condiciones de ultra alto vacío (UHV), debido a que a presiones mayores la tasa de adsorción de contaminantes sobre la muestra puede ser del orden de varias monocapas atómicas, impidiendo el estudio de la superficie que realmente se quiere analizar. 
Los espectros XPS fueron obtenidos en UHV con un espectrómetro XR50 Specs GmbH con Mg K (a) como fuente de excitación y un analizador de energía de media esfera PHOIBOS 100. Se calibró con Au 4f7/2 y Cu 2 p3/2 con energías de enlace de 84,00 y $933,67 \mathrm{eV}$, respectivamente. A su vez, debido a que la muestra puede adquirir una carga estática durante las medidas, se empleó como patrón interno la señal $C$ 1s con energía de enlace $284.6 \mathrm{eV}$. Para la deconvolución de los espectros resultantes se empleó el software XPSpeak4.1, usando funciones gaussianas - lorentzianas, corroborando la calidad del ajuste empleando la prueba $X^{2}$.

La preparación de las muestras se efectuó depositando lentamente gotas de dispersiones de las Si nps en algún solvente volátil sobre un sustrato de Au (en el caso de medidas de alta resolución), previamente tratado con solución piraña. En el caso de necesitar solo medidas para cuantificación, se usaron soportes de C. La cantidad de muestra utilizada sobre el sustrato corresponde a la mínima necesaria para la apreciación a simple vista de un depósito de aspecto claro.

\subsection{Microscopía de transmisión de electrones (TEM)}

Las imágenes obtenidas por TEM fueron realizadas usando diversos equipos:

- Las imágenes presentadas en el capítulo 4 fueron tomadas empleando un microscopio Philips CM-10.

- Las micrografías del capítulo 5, 6 y 7 con un equipo JEOL 2010 F.

- Las imágenes a bajos aumentos del capítulo 7 se tomaron usando un microscopio JEOL 1200 EX II.

En todos los casos, las muestras fueron preparadas sumergiendo las grillas (Ted Pella ${ }^{\circledR}$, Lacey Carbon typeA, 300 mesh) en suspensiones de las distintas nps, usando pinzas antiadherentes para microscopía electrónica. Luego de sumergidas, las grillas fueron colocadas en papel de filtro y secadas bajo corriente de Argón 0 Nitrógeno. El análisis estadístico de las imágenes obtenidas se realizó empleando el software libre ImageJ.

\subsection{Espectroscopía de emisión estacionaria y resuelta en el tiempo}

Para comprender las vías radiativas de decaimiento de una especie excitada es necesario obtener experimentalmente una función vinculada con la densidad de probabilidad para las transiciones desde el estado vibracional fundamental del estado electrónico excitado (En general, este es el singlete de menor energía, $\mathrm{S}_{1}$ ) hacia los diversos estados vibracionales del estado electrónico fundamental $\left(\mathrm{S}_{0}\right)$. Esta densidad de probabilidad es la función $F\left(\lambda_{\text {em }}\right)$ denominada espectro de emisión, que es característico de cada compuesto. A su vez, la medida experimental que se obtiene que está relacionada con $\mathrm{F}\left(\lambda_{\mathrm{em}}\right)$ es la intensidad de emisión $\mathrm{I}_{\mathrm{E}}\left(\lambda_{\mathrm{em}}\right)$, que es proporcional a $F\left(\lambda_{\text {em }}\right)$ y al número de fotones absorbidos a la longitud de onda $\lambda_{\text {exc }}$. Este último valor puede ser remplazado por la intensidad absorbida por la muestra $I_{A}\left(\lambda_{\text {exc }}\right)$, definida en la ecuación (2.2). Por lo tanto, la intensidad de emisión puede ser escrita como:

$$
I_{E}\left(\lambda_{\text {em }}, \lambda_{\text {exc }}\right)=k F\left(\lambda_{\text {em }}\right) I_{A}\left(\lambda_{\text {exc }}\right)=k F\left(\lambda_{\text {em }}\right)\left[I_{0}\left(\lambda_{\text {exc }}\right)-I_{T}\left(\lambda_{\text {exc }}\right)\right]
$$

Donde k es un factor de proporcionalidad que depende de la configuración experimental del equipo y del ancho de banda del monocromador. Además, usando (2.1) puede expresarse (2.6) como:

$$
I_{E}\left(\lambda_{\text {em }}, \lambda_{\text {exc }}\right)=k F\left(\lambda_{\text {em }}\right) I_{0}\left(\lambda_{\text {exc }}\right)\left[1-10^{-A\left(\lambda_{\text {exc }}\right)}\right]
$$

Los espectros de excitación, emisión y las matrices de excitación - emisión a temperatura ambiente se obtuvieron usando un equipo Horiba Jovin-Yvon Spex Fluorolog FL3-11 Este equipo está diseñado de forma modular y montado en configuración T, como se esquematiza en la figura 2.1 (a). La RE usada para la excitación puede provenir desde $\mathrm{LS}_{1,2}$, las cuales son una lámpara continua de Xe de $450 \mathrm{~W}$ o una lámpara pulsada de Xe respectivamente, 0 desde $\mathrm{LS}_{3}$, donde pueden colocarse LEDs pulsados intercambiables. El haz de excitación puede ser polarizado y enfocado usando $P_{j}$ y $L_{j}$, que en el caso de $j=1$ se controlan de forma automática mientras que en $\mathrm{j}=4$ manualmente. A su vez, la RE proveniente de $L S_{1,2}$ pasa por un monocromador 
de doble rejilla $\left(\mathrm{MC}_{1}\right)$ para la selección de la longitud de onda de excitación que incide sobre la muestra (S). La emisión de $\mathrm{S}$ puede ser colectada en 2 caminos de detección distintos. Para la detección en el NIR, la emisión se enfocó con $\mathrm{L}_{2}$ en un monocromador de doble rejilla $\left(\mathrm{MC}_{2}\right)$, y la $R E$ proveniente de este último se dispersó sobre un detector de InP/InGaAsP sensible en el NIR. En el rango UV-vis se utilizó $L_{3}$ para enfocar sobre otro en un monocromador de doble rejilla $\left(\mathrm{MC}_{3}\right)$, y el haz de salida fue redirigido usando un espejo móvil $\left(\mathrm{M}_{1}\right)$ hacia un tubo fotomultiplicador (PMT) para medidas en estado estacionario o un fotodiodo de avalancha (APD) en medidas resueltas en el tiempo. En ambos caminos de detección se dispone de polarizadores $\left(\mathrm{P}_{2}\right.$ y $\left.\mathrm{P}_{3}\right)$ que se controlan de forma automática.

Todos los espectros fueron adquiridos con $1 \mathrm{~nm}$ de ancho de rejilla de entrada y de salida, incrementos de longitud de onda de barrido de $1 \mathrm{~nm}$, tiempo de integración en cada paso de 0,1 segundos y se corrigieron por la sensibilidad dependiente del detector y la fuente usando los espectros de patrones de calibración Cumarina 115 y 9,10-DPA en ciclohexano [1] y por la dispersión Raman usando el espectro del solvente.

Para estimar los rendimientos cuánticos de emisión $\Phi_{\mathrm{PL}, \mathrm{X}}$ se empleó el método del patrón [6]. Este consiste en comparar el analito de interés con un compuesto estándar para la región de detección de interés, empleando las mismas condiciones de excitación y detección (energía de la fuente de excitación a la longitud de onda de excitación empleada, ancho de rendijas, región de detección, voltaje bias del detector, etc).

$$
\Phi_{P L, X}=\Phi_{P L, R} \frac{n_{X}^{2}}{n_{R}^{2}}\left(\frac{\int_{\lambda_{e m, 1}}^{\lambda_{e m, 2}} F_{X}\left(\lambda_{e m}, \lambda_{e x}\right) d \lambda}{\int_{\lambda_{e m, 1}}^{\lambda_{e m} 2} F_{R}\left(\lambda_{e m}, \lambda_{e x}\right) d \lambda}\right)=\Phi_{P L, R} \frac{n_{X}^{2}}{n_{R}^{2}}\left(\frac{\int_{\lambda_{e m, 1}}^{\lambda_{e m, 2}} I_{X}\left(\lambda_{e m}, \lambda_{e x}\right) d \lambda}{\int_{\lambda_{e m, 1}}^{\lambda_{e m} 2} I_{R}\left(\lambda_{e m}, \lambda_{e x}\right) d \lambda}\right)\left(\frac{1-10^{-A_{R}\left(\lambda_{e x c}\right)}}{1-10^{-A_{X}\left(\lambda_{e x c}\right)}}\right)
$$

Donde los subíndices $\mathrm{X}$ y $\mathrm{R}$ denotan al analito y la referencia, respectivamente, $\mathrm{n}$ el índice de refracción, la

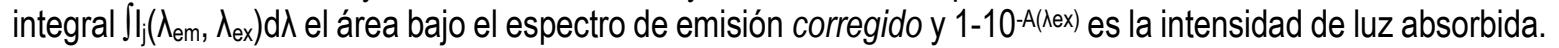
Para las medidas de $\Phi_{P L}$ se trabajó en condiciones de $\mathrm{A}\left(\lambda_{\mathrm{ex}}\right)<0.05$, para evitar distorsiones por filtro interno. Se emplearon diversos estándares, según la región de excitación. Los más usados fueron los siguientes:

\begin{tabular}{cccc}
\hline Reactivo & Emisión $(\mathrm{nm})$ & $\boldsymbol{\Phi}_{\mathrm{PL}, \mathrm{R}}$ & Solvente \\
\hline Naftaleno & $300-400$ & $0.23 \pm 0.02$ & Ciclohexano \\
Triptófano & $300-380$ & $0.14 \pm 0.02$ & Agua $(\mathrm{pH} 7.2)$ \\
Antraceno & $360-480$ & $0.27 \pm 0.03$ & $\mathrm{EtOH}$ \\
9, 10- difenil antraceno & $400-500$ & $0.90 \pm 0.02$ & Ciclohexano \\
(9,10- DPA) & $400-600$ & $0.55 \pm 0.03$ & $\mathrm{H}_{2} \mathrm{SO}_{4(\mathrm{ac})} 0.5 \mathrm{M}$ \\
Sulfato de quinina & & &
\end{tabular}

Tabla 2.1. Estándares de emisión utilizados.

En experimentos de anisotropía de emisión, tanto estacionaria como resuelta en el tiempo, los polarizadores empleados fueron calibrados usando dispersiones de sílice coloidal (Ludox ®, Sigma Aldrich) y soluciones de triptófano (Sigma Aldrich, >98\%) cuyos valores de anisotropía de emisión son conocidos y se encuentran bien documentados en literatura [6].

Las medidas resueltas en el tiempo se realizaron bajo la metodología de conteo de fotones individuales TCSPC (del inglés Time Correlated Single Photon Counting). Se emplearon distintas configuraciones experimentales para estos experimentos:

- Empleando el mismo espectrofluorómetro Horiba Jovin-Yvon Spex Fluorolog FL3-11 disponible en INIFTA, adosando un detector apropiado para experimentos de TCSPC. Las fuentes de excitación empleada fueron LEDs pulsados intercambiables nanoLED ${ }^{\circledR}$ (Horiba Scientific). Se utilizaron LEDs 
de 295 nm (FWHM < 1 ns), 341 nm (FWHM 400 ps), 388 nm (FWHM <1.2 ns), 461 nm (FWHM <1.2 ns) operando a $1 \mathrm{MHz}$ de repetición. La emisión de las muestras se detectó en ángulo mágico $\left(54.7^{\circ}\right)$ y fue dispersada en un monocromador y colectada por un fotodiodo de avalancha (APD). Este montaje se esquematiza en la figura 2.1 (a). Las señales fueron convertidas usando un convertidor tiempoamplitud (TAC) con rango de $50 \mathrm{~ns}$. Los IRF para cada fuente de excitación se determinaron siguiendo las recomendaciones del fabricante, para lo que se utilizaron suspensiones de sílice coloidal Ludox ${ }^{\circledR}$ (SM-30, $30 \% \mathrm{SiO}_{2}$ en agua a pH 10,2 con un diámetro de partícula medio de $7 \mathrm{~nm}$, área superficial: $345 \mathrm{~m} 2 / \mathrm{g}$, Sigma-Aldrich).

- Utilizando un arreglo experimental de diseño personalizado sobre un banco óptico, montado en el laboratorio de los Profesores Moore, Moore y Gusta en Arizona State University (ASU). Se emplearon distintas fuentes de excitación, según la necesidad del experimento:

Para experimentos de alta resolución temporal se usó un láser de Titanio:Zafiro (Ti:S, LS 2 ) como fuente de excitación en la región de 740 - 900 nm (Spectra Physics, Tsunami bombeado por un Milennia) con pulsos de 130 fs operando a $80 \mathrm{MHz}$. Para obtener excitación en el rango de 370 a $450 \mathrm{~nm}$, el haz de salida se dirigió a un doblador y selector de pulsos (Spectra Physics, modelo 3980). El haz de excitación se acondicionó usando filtros pasa banda adecuados $\left(\mathrm{F}_{2}\right)$ y polarizador $\left(\mathrm{P}_{2}\right)$.

En experimentos con menor resolución temporal o con excitación en rangos que el Ti:S no era viable, se utilizó como fuente un láser supercontinuo de fibra óptica, que consiste en un láser de fibra con mode locking pasivo y un cristal fotónico de alta no linealidad que actúa como generador del supercontinuo (Fianium SC450, LS 1 ). La láser entrega pulsos de 6 ps a velocidades de repetición variable entre 0.1 y $40 \mathrm{MHz}$. El haz de salida se dirigió a un filtro de modulación acusto-óptico (Fianium AOTF) para obtener pulsos de excitación en longitudes de onda controlada entre $450-900 \mathrm{~nm}$. El haz de excitación se acondicionó usando filtros pasa banda adecuados $\left(F_{1}\right)$ y polarizador $\left(P_{1}\right)$.

La emisión se detectó en ángulo mágico $\left(54.7^{\circ}\right.$ controlado con $\left.\mathrm{P}_{3}\right)$ usando un monocromador de doble rejilla (Jovin-Yvon Gemini-180, MC) y detectando con un tubo fotomultiplicador de microcanales (Hamamatsu R3809U-50, MCP). El IRF es de $35-55$ ps, según la longitud de onda de excitación. El espectrómetro se controló usando un software desarrollado sobre LabView, y la adquisición de datos se realizó usando una placa de conteo de fotones individuales (Becker-Hickl, SPC-830). El diagrama de este experimento de TCSPC se esquematiza en la figura 2.1 (b).

Los ajustes de los decaimientos de emisión resueltos en el tiempo y de espectros resueltos en el tiempo fueron realizados optimizando los valores de $\chi^{2}\left(1<\chi^{2<1.25)}\right.$ para las curvas individuales en el primer caso, y usando análisis global para el segundo. Los ajustes se realizaron con los softwares DAS6 Analysis, Glotaran o ASUfit.

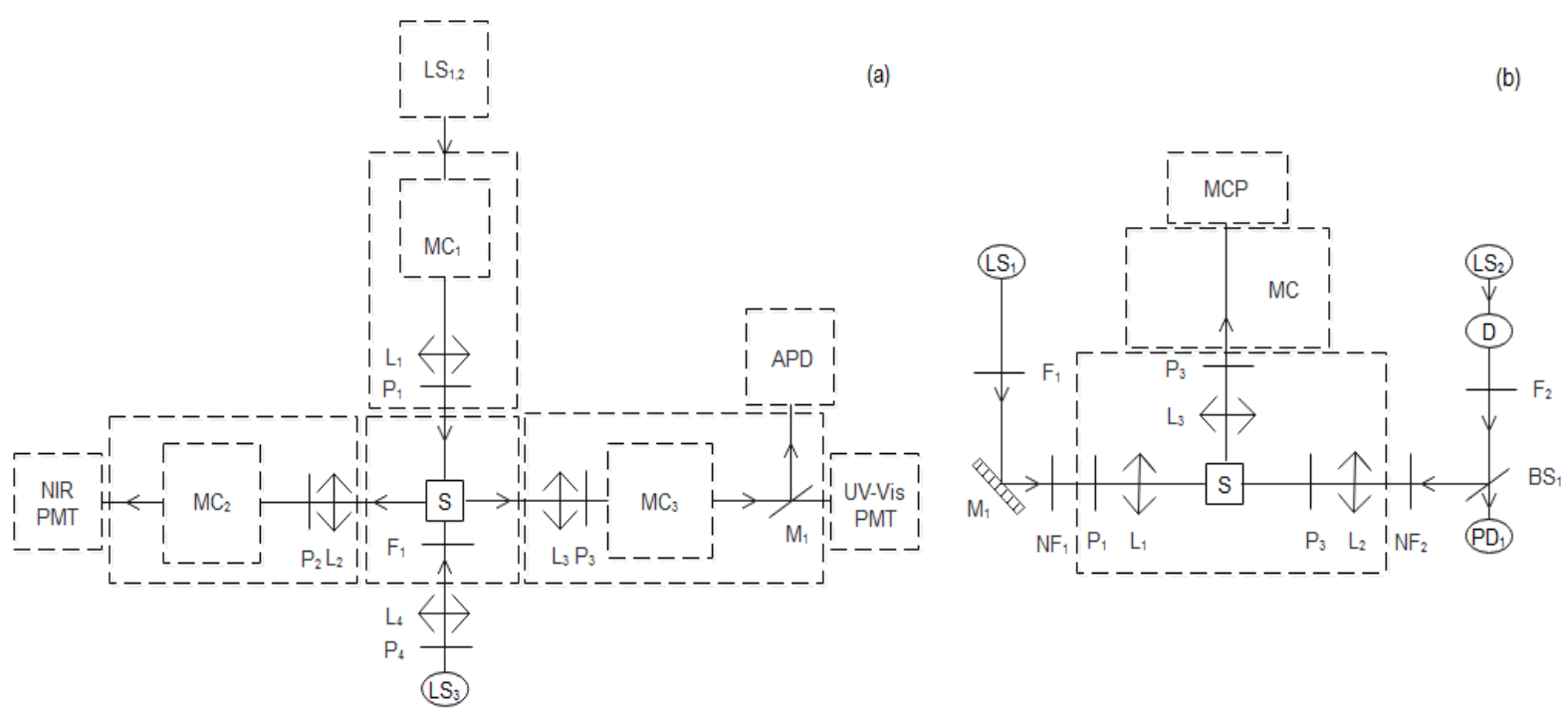

Figura 2.1. Montajes experimentales para experimentos de emisión (A) Experimento disponible en INIFTA (B) Montado en el laboratorio de los profesores Moore y Gust, en ASU. 


\subsubsection{Principios básicos}

La emisión de luz de una sustancia recibe el nombre general de luminiscencia, y ocurre desde estados electrónicamente excitados. La luminiscencia se divide formalmente en dos categorías -fluorescencia y fosforescencia- dependiendo de la naturaleza del estado excitado. En estados excitados singlete, el electrón en el orbital excitado está apareado (por spin opuesto) al segundo electrón en el orbital del estado basal. Consecuentemente, retorna al estado basal rápidamente por emisión de un fotón. Las velocidades de emisión de fluorescencia son típicamente $10^{8} \mathrm{~s}^{-1}$, de modo que los típicos tiempos de vida de fluorescencia están cercanos a los $10 \mathrm{~ns}\left(10^{*} 10^{-9} \mathrm{~s}\right)$. Sin embargo, muchos fluoróforos presentan tiempos de vida del orden de los sub-nanosegundos. La fosforescencia es la emisión de luz desde un estado excitado triplete, en el cual el electrón en el orbital excitado tiene la misma orientación de spin que el electrón en el estado basal. La transición al estado basal está prohibida y la velocidad de emisión es lenta $\left(10^{-3}\right.$ a $\left.10^{3} \mathrm{~s}^{-1}\right)$, tal que los tiempos de vida son típicamente de milisegundos a segundos. Las moléculas con 'átomos pesados como bromo o iodo suelen presentar fosforescencia, por permitir el cruce entre sistemas para lograr la inversión del spin. La fosforescencia usualmente no se observa en solución a temperatura ambiente, dado que existen muchos procesos de desactivación que compiten con la emisión, tal como decaimientos no radiativos o procesos de extinción (quenching).

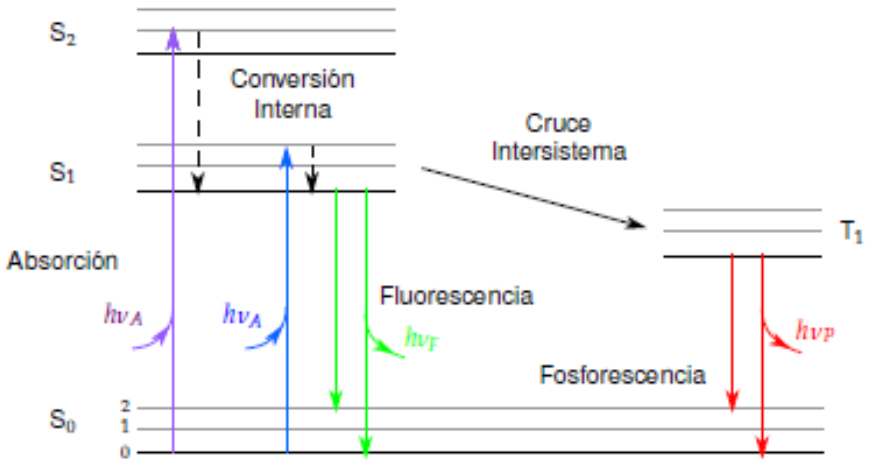

(a)

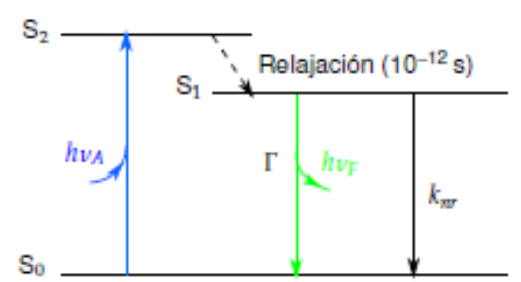

(b)

Figura 2.1. (a) Ejemplo de un diagrama de Jablonski, adaptado de la referencia [1], (b) diagrama de Jablonski simple donde solo se considera la fluorescencia.

Estos procesos de absorción y emisión de luz son generalmente representados mediante un diagrama de Jablonski, como el de la figura 2.11a. Estos diagramas, muestran que típicamente en fluorescencia la energía de emisión es un poco menor que la energía de absorción. A este fenómeno se lo denomina "corrimiento de Stokes (Stokes Shift).

Además del espectro de emisión, el tiempo de vida de fluorescencia y el rendimiento cuántico son quizás las características más importantes de los fluoróforos. El rendimiento cuántico de fluorescencia esté definido como el número de fotones emitidos relativo al número de fotones absorbidos. Sustancias con una un gran rendimiento cuántico, cercano a la unidad, tales como rodaminas, muestran emisiones muy brillantes. El tiempo de vida es también importante, ya que determina el tiempo disponible para que el fluoróforo interactúe con su ambiente, y proporcione información desde su emisión. El significado del rendimiento cuántico y del tiempo de vida se representan en el diagrama simplificado de la figura $2.11 \mathrm{~b}$, donde solo se hace referencia a los procesos que están relacionados con retorno al estado fundamental. En particular se resalta la velocidad de emisión de fluorescencia $\left(\mathrm{k}_{\mathrm{r}}\right)$ y la velocidad de decaimiento del j-ésimo canal no radiativo $\left(\mathrm{k}_{\mathrm{nr}, \mathrm{j}}\right)$ hacia $\mathrm{S}_{0}$. Estos parámetros están relacionados con el rendimiento cuántico, $\Phi_{\mathrm{PL}}$ : 


$$
\Phi_{P L}=\frac{k_{r}}{k_{r}+\sum_{i} k_{n r, i}}
$$

donde se puede ver que cuanto menor es el decaimiento por canales no radiativos respecto del decaimiento de emisión de fluorescencia, esto es $\mathrm{k}_{\mathrm{r}} \gg \sum \mathrm{k}_{\mathrm{nr} r \mathrm{i}}, \Phi_{\mathrm{PL}}$ se acerca a la unidad. Nótese que el rendimiento cuántico de fluorescencia es siempre menor o igual a la unidad debido a las pérdidas de Stokes (origen del corrimiento de Stokes).

El tiempo de vida del estado excitado está definido por el promedio de tiempo que las moléculas están en el estado excitado antes de retornar al estado basal. Para el diagrama simple de la figura $2.11 \mathrm{~b}$ el tiempo de vida es

$$
\tau_{P L}=\frac{1}{k_{r}+\sum_{i} k_{n r, i}}
$$

Si bien la emisión en estado estacionario es la forma más ampliamente difundida de esta espectrometría, la emisión resuelta en el tiempo aporta información complementaria a la primera que resulta de mucha utilidad. En la primera, la muestra es irradiada con una fuente de iluminación constante. En cambio, la muestra puede ser irradiada con un pulso de luz, donde el ancho del pulso es típicamente más corto que el tiempo de decaimiento de la muestra. Este decaimiento de la intensidad es colectado con un sistema de detección apropiado para la escala de tiempo de ns (o menor). En general si un fluoróforo presenta un decaimiento simple, este puede describirse como

$$
I(t)=I_{0} e^{-t / \tau_{P L}}
$$

La señal de decaimiento obtenida, es una convolución del pulso de luz y el decaimiento de fluorescencia propio del fluoróforo. Por lo que se debe resolver la integral de convolución de alguna forma para poder realizar un ajuste adecuado de los datos.

$$
N(t)=I R F(t) \otimes I(t)
$$

donde $\mathrm{N}$ es la señal colectada, IRF es la función respuesta del instrumento e I es la intensidad de fluorescencia dependiente del tiempo.

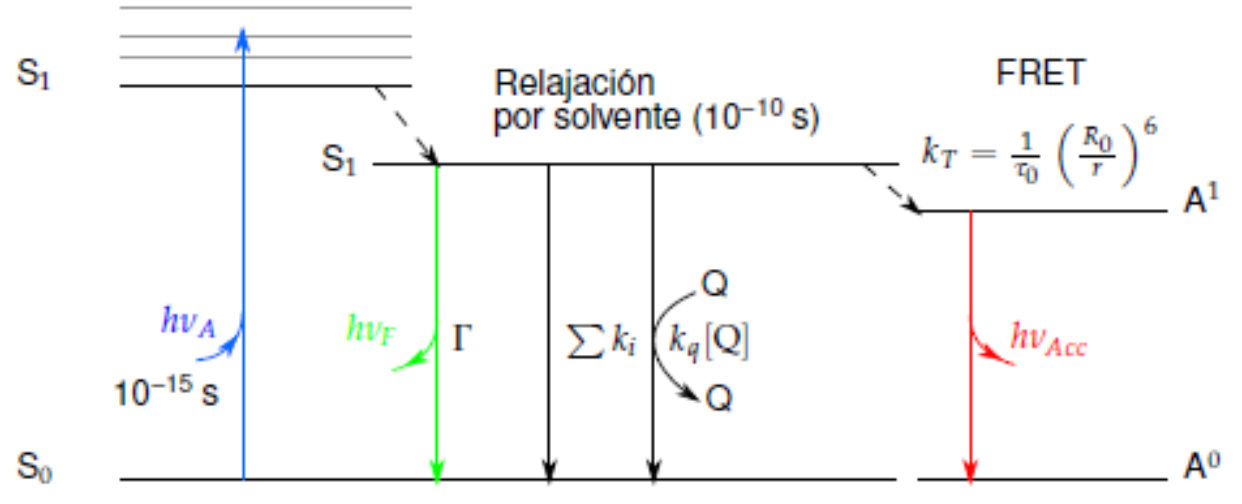

Figura 2.2. Diagrama de Jablonski con quenching colisional $(Q)$ o por transferencia de energía por resonancia (FRET). El termino $\sum \mathrm{k}_{n, \mathrm{i}}$ es usado para representar los caminos no radiativos por los que se alcanza el estado basal. 
La intensidad de fluorescencia puede verse disminuida por una gran variedad de procesos. Esta disminución de la intensidad es llamada extinción o quenching. El quenching puede ocurrir por diferentes mecanismos. El quenching colisional ocurre cuando el fluoróforo en el estado excitado es desactivado luego del contacto con alguna otra molécula en solución, la cual es llamada extintor o quencher, como se ejemplifica en el diagrama de Jablonski modificado en la figura 2.12. En estos procesos las moléculas no son alteradas químicamente. La disminución de la intensidad por quenching colisional esta descripta por la ecuación de Stern - Volmer:

$$
\frac{I_{0}}{l}=1+\mathrm{K}_{\mathrm{SV}}[\mathrm{Q}]=1+\mathrm{k}_{\mathrm{q}} \tau_{0}[\mathrm{Q}]
$$

En esta ecuación, $\mathrm{K}_{\mathrm{sv}}$ es la constante de quenching de Stern-Volmer, $\mathrm{k}_{\mathrm{q}}$ es la constante de quenching bimolecular, y [Q] es la concentración del quencher. La constante $K_{s v}$ indica la sensibilidad del fluoróforo respecto a un determinado quencher.

\subsubsection{Transferencia de energía no radiativa (FRET)}

El fenómeno de transferencia de energía no radiativa es muy común en la naturaleza. Por ejemplo, la fotosíntesis requiere la transferencia eficiente de energía desde la absorción de fotones por un complejo antena hacia el núcleo de producción de ATP. Esto permite a las células utilizar eficientemente un rango mayor del espectro que si se limita solo la absorción de la clorofila. La energía es transferida vía FRET, una transferencia de energía no trivial desde una molécula $D^{*}$ electrónicamente excitada a una molécula aceptor $A$, a través del acoplamiento de los dos respectivos dipolos oscilantes. Las moléculas de aceptor pueden luego decaer al estado basal a través de vías radiativas o no radiativas. El tratamiento matemático riguroso fue mostrado por Theodor Förster en 1948 en Gotinga, Alemania, y es por esto que el fenómeno recibe el nombre de transferencia de energía de resonancia de Förster, FRET - de sus siglas en inglés Förster Resonance Energy Transfer.

DONOR

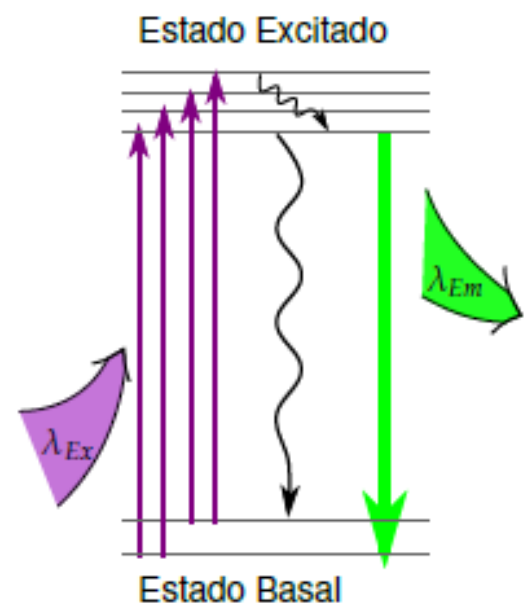

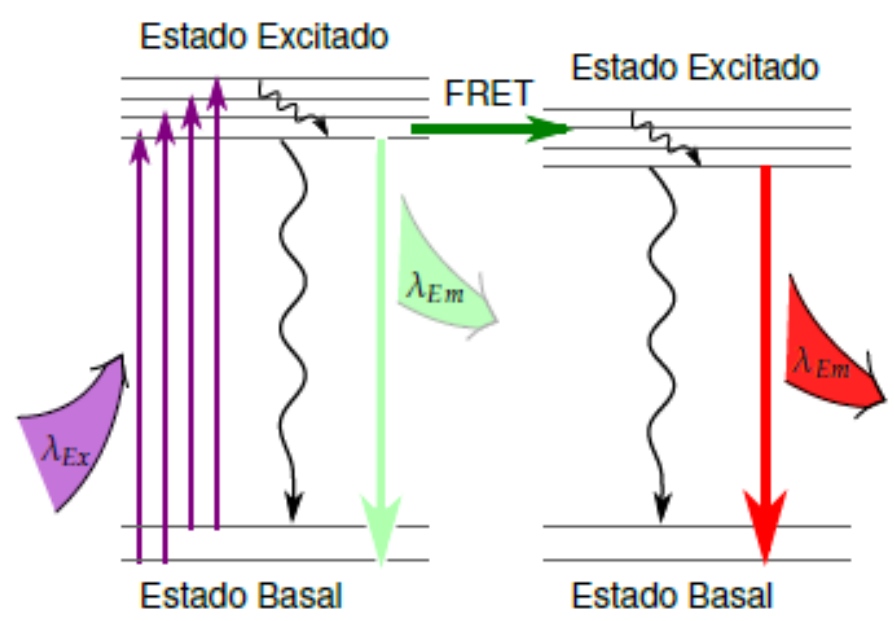

Figura 2.3. Diagrama de Jablonski de un donor que emite en forma independiente y de un sistema donor-aceptor con FRET.

Cuando el donor (D) es excitado por un fotón, puede revertir al estado basal a través de la emisión de un fotón. El tiempo de vida de fluorescencia depende de la disponibilidad del camino de decaimiento y el FRET adiciona otra posible vía de decaimiento por un proceso no-radiativo. El tiempo de vida de fluorescencia del donor es 
entonces más corto cuando experimenta FRET que cuando hay ausencia de un aceptor (A). La eficiencia de la transferencia de energía puede ser expresada y determinada como sigue [1]

$$
E_{E T}=1-\frac{I_{D A}}{I_{D}}
$$

donde $I_{D A}$ y $I_{D}$ es la intensidad de fluorescencia del donor en presencia y ausencia del aceptor FRET. También, se puede determinar la eficiencia FRET mediante espectroscopia de fluorescencia resuelta en el tiempo:

$$
E_{E T}=1-\frac{\tau_{D A}}{\tau_{D}}
$$

donde $\mathrm{E}$ es la eficiencia de FRET, TDA es el tiempo de vida de fluorescencia del donor en presencia de la transferencia de energía y, TD es el tiempo de vida de fluorescencia sin transferencia de energía. La eficiencia es función de la distancia donor-aceptor, que puede ser expresada como:

$$
E_{E T}=\frac{1}{1+\left(r_{D A} / R_{0}\right)^{6}} \wedge \mathrm{k}_{E T}=\frac{1}{\tau_{0}}\left(R_{0} / r_{D A}\right)^{6}
$$

donde $r_{D A}$ es la distancia donor-aceptor, y $R_{0}$ es la distancia de Förster, la cual está definida como la distancia $D-A$ a la cual $E=0.5$. $R_{0}$ puede ser calculada por la siguiente ecuación:

$$
R_{0}^{6}=\mathrm{C}_{0} \mathrm{k}^{2} \mathrm{Jn}^{-4} \Phi_{\mathrm{PL}, \mathrm{D}}
$$

donde $\mathrm{C}_{0}$ es una constante $\left(8.79\right.$ * $10^{-5}$ si $\mathrm{R}_{0}$ esta expresada en $\left.\mathrm{A}\right), \mathrm{k}^{2}$ es un factor de orientación de los dipolos, $\mathrm{n}$ es el índice de refracción promedio del medio en el cual la transferencia de energía ocurre, $\Phi_{\mathrm{PL}, \mathrm{D}}$ es el rendimiento cuántico en ausencia de un aceptor, y J es la integral de solapamiento espectral de la emisión del donor y la absorbancia del aceptor [1]. La figura 2.4 muestra la dependencia entre la eficiencia de un proceso FRET y la distancia de separación entre el donor y el aceptor. Las líneas punteadas marcan $\mathrm{R}_{0}$. 


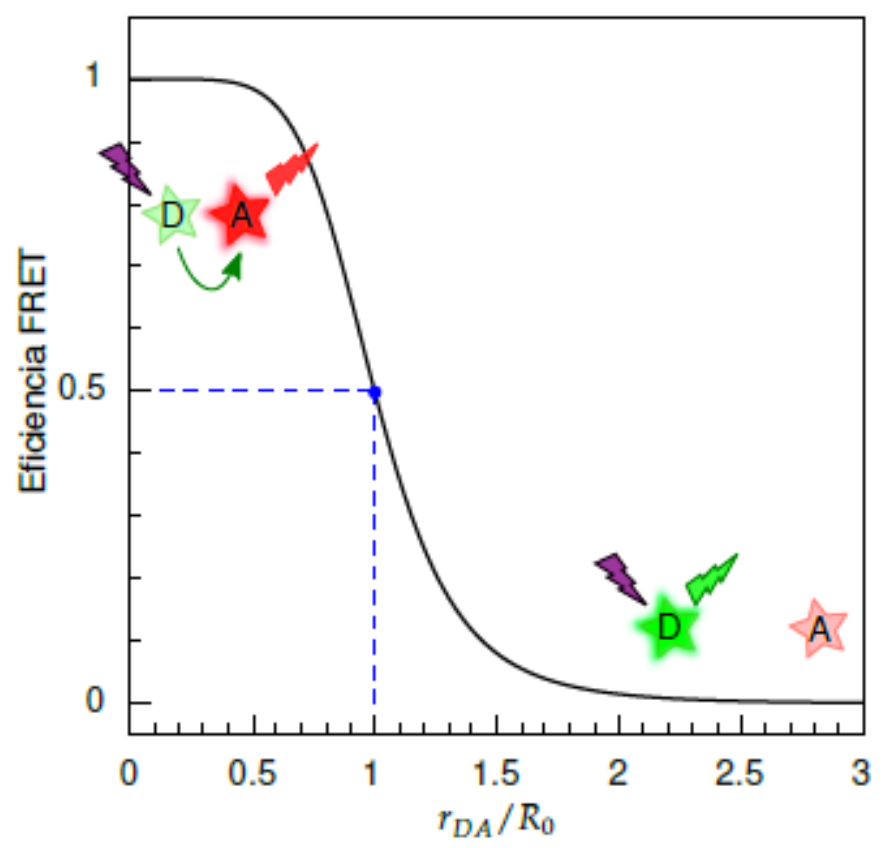

Figura 2.4. Eficiencia de FRET en función de la distancia donor-aceptor.

El factor de orientación juega un rol principal en la transferencia de energía, pero es difícil de conocer precisamente, excepto en entornos muy rígidos. Los valores de $\mathrm{K}^{2}$ pueden tomar valores entre 0 y 4 , pero se suele asumir el valor 2/3 en una situación de libre rotación y orientación al azar del par donor-aceptor [1].

Al momento de seleccionar un par FRET es de fundamental importancia la integral de solapamiento J,

$$
J=\frac{\int_{0}^{\infty} I_{D}(\lambda) \varepsilon_{A}(\lambda) \lambda^{4} d \lambda}{\int_{0}^{\infty} I_{D}(\lambda) d \lambda}
$$

donde $I_{D}(\lambda)$ es el espectro de fluorescencia del donor y $\varepsilon_{A}(\lambda)$ es el coeficiente de extinción del aceptor. En caso en que no todos los donores cuenten con un aceptor (marcado incompleto), se obtienen una mayor intensidad de fluorescencia y, por lo tanto, al determinar la eficiencia FRET ( $\left.E_{E T}\right)$ se comete un error por defecto. Al emplear estos valores para calcular la distancia donor-aceptor, $\mathrm{r}_{\mathrm{DA}}$, se comete un error por exceso al obtener separaciones mayores a las reales. En caso de conocer la fracción de aceptor por cada donor $\left(\mathrm{f}_{\mathrm{A}}\right)$, la eficiencia de transferencia de energía se calcula como

$$
E_{E T}=\left(1-\frac{I_{D A}}{I_{D}}\right) \frac{1}{f_{A}}
$$

La mayoría de los valores de $R_{0}$ están en el rango de los Angstroms (A), y como se puede ver de la ecuación 2.36, la eficiencia decae con la distancia donor-aceptor a la sexta potencia. Por esto FRET es una herramienta extremadamente sensible para determinar distancias en el rango de los nanómetros, por lo que puede ser usada como una excelente "regla molecular". Por esto, FRET ha sido ampliamente utilizado para estudiar diferentes sistemas complejos, como interacción entre proteína - nucleótido, proteína - proteína, plegamiento de proteínas, entre otros. 


\subsubsection{Transferencia de carga}

Las reacciones de transferencia de carga son ubicuas en la naturaleza, por ejemplo, el proceso de separación de cargas es uno de los pasos más importantes de la fotosíntesis, involucrado en la transformación de energía solar en potencial químico. Esta estrategia inspiró distintos enfoques que hoy en día se denominan fotosíntesis artificial [7]. Estos involucran el diseño y ensamblado de sistemas capaces de producir combustibles solares, celdas de combustible fotoelectroquímicas, e ingeniería de enzimas de organismos fotoautotróficos. A su vez, la nanotecnología contribuyó a este campo con nuevos materiales activos para conversión de energía y buscando el re-diseño de sistemas para aumentar su eficiencia [8]. Entender la fisicoquímica detrás de estas reacciones fue un proceso largo, que incluyó un trabajo mancomunado entre la electroquímica y la fotoquímica [9] y culminó con los trabajos de Marcus sentando las bases teóricas para comprender el proceso [10]-[12].

Las propiedades redox de las moléculas pueden ser mejoradas en el estado excitado. En estos casos, las reacciones proceden según las siguientes reacciones:

$$
\begin{aligned}
& { }^{1} D+A \rightarrow D^{+}+A^{-} \\
& { }^{1} A+D \rightarrow A^{-}+D^{+}
\end{aligned}
$$

Donores (D) típicos son la trimetilamina, naftaleno, antraceno, perileno, fenantreno, etc; mientras que aceptores (A) ampliamente usados son las quinonas o el 9,10 - dicianoantraceno.

En reacciones en solución y en caso de que esta no sea controlada por difusión, puede determinarse la constante de transferencia de carga $k_{e T}[1]$, [6]. Hay 2 casos posibles:

- Si la interacción entre el donor y el aceptor en el par reactivo es fuerte, este par reactivo forma un exciplejo y se estudia como tal.

- Si la interacción entre el donor y el aceptor es débil, la reacción puede estudiarse usando la teoría de Marcus.

La teoría de Marcus predice una dependencia cuadrática de la energía libre de activación con la energía libre estándar de reacción:

$$
\Delta \mathrm{G}^{\ddagger}=\left(\Delta \mathrm{G}^{0}+\lambda\right)^{2} / 4 \lambda
$$

Siendo $\lambda$ la energía de reorganización, que está dada por la suma de contribuciones de esfera interna (debido a cambios estructurales de las especies donoras y aceptoras) y externa (reorganización del solvente) [13].

Teniendo en cuenta la relación de $\mathrm{k}_{\mathrm{e}} \mathrm{con} \Delta \mathrm{G} \ddagger$, si se realiza un gráfico de ln $\mathrm{k}_{\mathrm{e}} \mathrm{T}$ Vs $\Delta \mathrm{G}^{0}$ se observa una parábola con máximo en $\Delta \mathrm{G}^{0}=-\lambda$. Si $\Delta \mathrm{G}^{0}>-\lambda$, In $\mathrm{k}_{\mathrm{e}}$ aumenta cuando $-\Delta \mathrm{G}^{0}$ aumenta, que es llamada región normal de Marcus, ya que se comporta según lo observado para reacciones en general. Sin embargo, en el caso de $\Delta G^{0}$ $<-\lambda$, In $k_{\text {et }}$ disminuye cuando $-\Delta G^{0}$ aumenta, lo cual constituyó un hecho completamente inesperado y se lo denominó región invertida de Marcus [14], [15].

\subsection{Espectroscopía de absorción UV-visible resuelta en el tiempo}

\subsubsection{Principios básicos}

La espectroscopia de absorción de transientes (TAS, del inglés Transient Absorption Spectroscopy) es una extensión de la espectroscopia de absorción estacionaria. Aquí, la absorbancia de una muestra, para una longitud de onda particular o para un rango de longitudes de onda, se mide en función del tiempo después de la excitación por un pulso de radiación electromagnética. En un experimento típico, tanto la luz de excitación, 
que suele llamarse pulso de bombeo o pump, como el pulso de análisis, llamado pulso de sondeo o probe, son generados por láseres pulsados [16], [17].

Dentro de las técnicas TAS, en fotoquímica suelen diferenciarse por cuestiones históricas 2 casos particulares:

- Experimentos de Laser Flash Fotólisis (LFP, del inglés Laser Flash Photolysis), en los cuales la ventana temporal de estudio va de los cientos de ns a los ms.

- Experimentos de fs-TAS, esto es, absorción de transientes resuelta en los femtosegundos, con una ventana de detección desde los fs a pocos ns. Dentro de estas técnicas, hay experimentos utilizando 2 (pump/probe), 3 (pump/dump/probe o pump/re-pump/probe, etc.) 04 (photon echo, four wave mixing, etc.) pulsos de radiación electromagnética [18].

En esta tesis utilizamos experimentos de fs-TAS en configuración pump/probe (los cuales llamaremos simplemente TAS). En este experimento, la muestra es excitada por un pulso de bombeo que marca $t=0$, y la evolución temporal del estado excitado generado se monitorea aprovechando su interacción un pulso retardado. Las características de estos dos pulsos (extensión temporal y espectral, forma y fase, potencia, etc.) determinará que estados excitados y que propiedades podemos sondear. Las principales ventajas de fs-TAS reside en la posibilidad de estudiar estados silenciosos en espectroscopía de emisión resuelta en el tiempo, y en la variedad de procesos no radiativos que pueden ser estudiados. En general, el análisis de datos de fs-TAS no puede ser realizado considerando estados estacionarios solución de la ecuación de Schrödinger para el sistema estudiado, sino que deben contemplarse paquetes de onda que evolucionan en la ventana temporal empleada (Esto es estrictamente necesario en los primero 100 ps del experimento) [17].

\subsubsection{Montaje Experimental}

La versatilidad de las técnicas de fs-TAS están sujetas a las capacidades del sistema óptico empleado, que determinan tanto la resolución temporal como espectral del experimento. Los sistemas usados en esta tesis se ajustan al expuesto en la figura 2.5 .

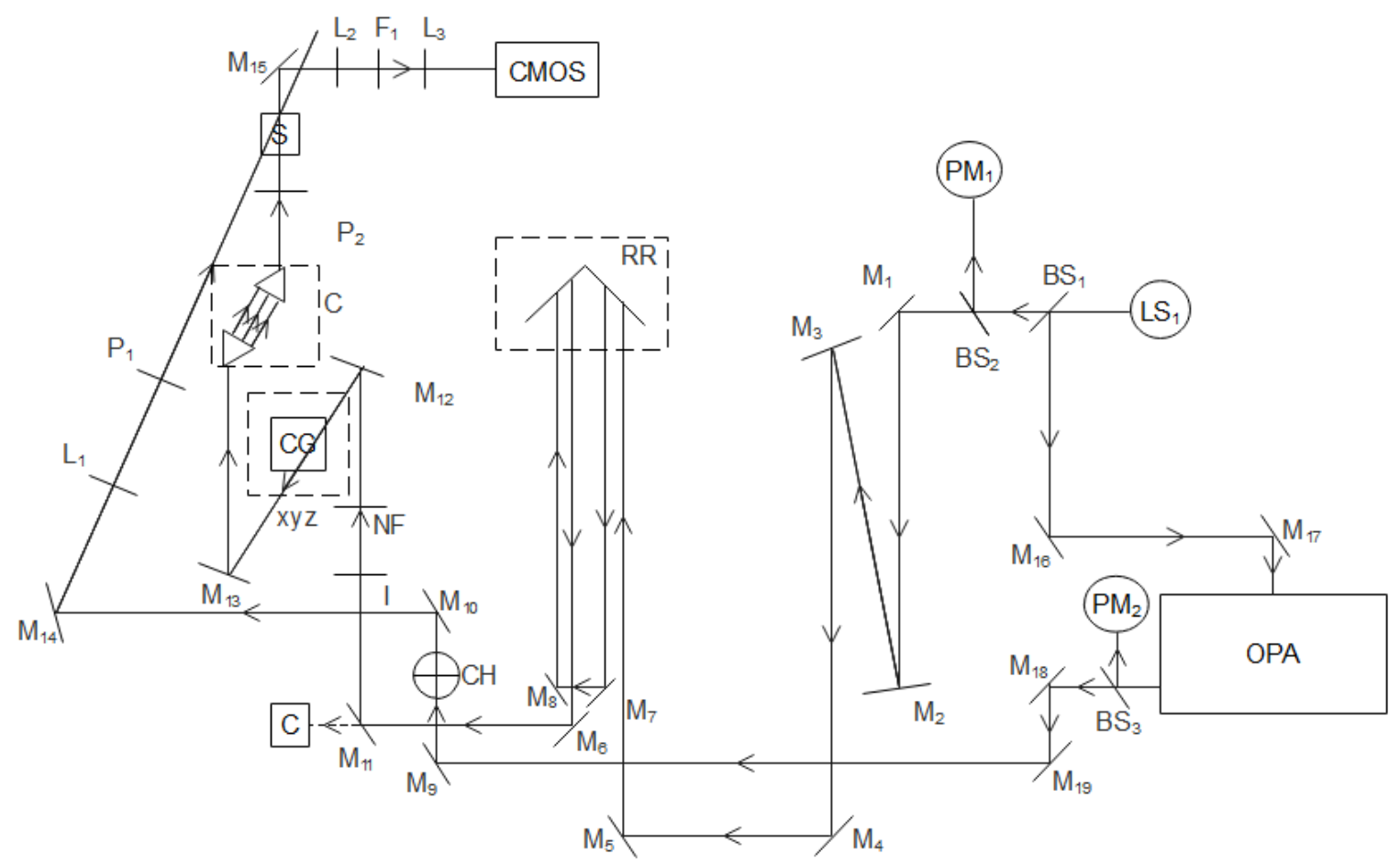

Figura 2.5. Configuración de los sistemas ópticos usados en experimentos de fs-TAS. 
Para los experimentos de fs-TAS usamos montajes en diversos laboratorios:

- Grupo de espectroscopía y microscopía de alta resolución, junto al Prof. Dr. Hodak en el Instituto de Química Física de los Materiales, Medio Ambiente y Energía (INQUIMAE UBA - CONICET).

- Department Chemie un Pharmazie, junto a la Prof. Dr. Carola Kryschi en Friedrich-Alexander Universität(FAU), Erlangen, Alemania.

- Department of Bioengineering, junto al Prof. Dr. Valentine Vullev en University of California at Riverside (UCR), Estados Unidos.

- Center of Biodesign, Department of Chemistry and Biochemistry, junto a la Prof. Dr. Su Lin y los Prof. Dr. Thomas Moore, Dra. Ana L. Moore y Dr. Devens Gusta, en Arizona State University (ASU), Estados Unidos.

Dado que el montaje utilizado en los 4 laboratorios es básicamente el mismo, describiremos en mayor profundidad el utilizado en ASU que fue el que más empleamos. El arreglo experimental se muestra en la figura 2.5, y la nomenclatura usa $L S_{i}$ para las fuentes láser, $M_{i}$ para espejos, $B S_{i}$ para divisores de haz, $\mathrm{PM}_{\mathrm{i}}$ para los medidores de energía de pulso, OPA para el amplificador óptico paramétrico, NF para los filtros neutros, F para los filtros de corte, $L$ para los lentes, $P$ para los polarizadores, RR para el conjunto de espejos que constituyen el retroreflector de la línea de retardo, $\mathrm{CH}$ para el selector de pulsos o chopper, C para la cámara de alineación, I para los iris, S para la muestra, CG para el medio activo de generación del supercontínuo de análisis y CMOS para el sistema de detección basado en junturas de oxidos metálicos y semiconductores.

Básicamente, el sistema laser ( $\left.\mathrm{LS}_{1}\right)$ consiste en un láser mode locked pulsado ( $<50 \mathrm{fs}$ ) de Ti:S sintonizable entre 740 y $800 \mathrm{~nm}$ Tsunami (Spectra Physics), bombeado por un láser Millenia CW de $532 \mathrm{~nm}$ (Spectra Physics). Los pulsos del Tsunami, que se utilizan como pulsos semilla, van a un amplificador regenerativo spitfire (Spectra Physics), que es bombeado por un láser Nd:YLF evolution (Coherent). El sistema completo opera a $1 \mathrm{kHz}$ y el ancho del pulso a mitad de altura en la salida es de aproximadamente $130 \mathrm{fs}$.

El haz de salida del amplificador spitfire es dividido en 2: Una parte del haz se dirige a un amplificador óptico paramétrico (OPA 800C, spectra Physics) en el cual, por procesos de óptica no lineal, se obtienen los pulsos de bombeo (pump), los cuales son monitoreados con un medidor de energía. Estos pulsos son seleccionados usando un chopper, y luego dirigidos a la muestra usando espejos y lentes y controlando su polarización usando $P_{1}$. La parte restante de la salida del spitfire va a un medidor de energía de pulsos para verificar la constancia de la calidad del haz de salida y al sistema óptico de retardo (compuesto por RR y los espejos $\mathrm{M}_{7}$ y $\mathrm{M}_{8}$ ). Luego pasa por una ventana de un material activo (usamos una ventana de zirconia) para generar el pulso de luz blanca (probe) que es controlada a usando filtros neutros, iris y un compensador $C$ formado por un arreglo de prismas, y finalmente incide en la muestra. Una vez atravesada la misma, la luz de análisis se dirige a través de filtros y lentes a una fibra óptica que conecta con el detector CMOS que se registra usando un boxcar. La resolución del equipo en su conjunto, es de $\sim 150$ fs. La potencia del haz de excitación en las distintas longitudes de onda empleadas medidas frente a la muestra se mantuvo cercana a $200 \mu \mathrm{W}$ [5], [19], [20].

\subsection{Análisis de datos}

El análisis de los resultados experimentales de este trabajo se realizó siguiendo métodos estadísticos estándar. Sin embargo, es importante mencionar y discutir dos técnicas usadas ampliamente en esta tesis: Análisis multivariante, aplicado a estudios espectroscópicos en estado estacionario; y análisis global y objetivo (Normalmente encontrados en bibliografía como global analysis y target analysis, respectivamente), los cuales se emplearon en el caso de experimentos espectroscópicos resueltos en el tiempo.

\subsubsection{Análisis multivariante}

El Análisis multivariante es un conjunto de métodos estadísticos y matemáticos, destinados a describir e interpretar los datos que provienen de la observación de varias variables estadísticas, estudiadas 
conjuntamente. En este trabajo de tesis, se utilizaron métodos de resolución de múltiples curvas por mínimos cuadrados alternados (MCR - ALS) y análisis de factores paralelos (PARAFAC).

En principio, los métodos MCR y PARAFAC están íntimamente relacionados con el análisis de componentes principales (PCA, del inglés Principal Component Analysis) y con la descomposición en valores singulares (SVD, del inglés Singular Values Decomposition).

La descomposición SVD de una matriz real o compleja es una factorización de la misma. Sea una matriz A de rango $r$ incluida en $R^{m \times n}$, tal que la matriz cuadrada $A^{t} A$ de $R^{n \times n}$ presenta $r$ autovalores $\lambda_{i}=\sigma_{i}^{2}$ y autovectores $\mathbf{V}_{i}$. A los valores $\sqrt{ } \lambda_{i}=\sigma_{i}$ se lo llama valores singulares de $\mathbf{A}$. Usando estos autovectores y valores singulares, puede probarse [21] que existe una factorización de la forma:

$$
A=U \Sigma V^{t}
$$

Donde $\boldsymbol{\Sigma} R^{m \times n}$ es la matriz diagonal de valores singulares ordenados de mayor a menor incluyendo los $r$ valores singulares y $n-r$ valores nulos, $\mathbf{V} R^{n x n}$ es una matriz cuyas columnas son los autovectores $\mathbf{V}_{i}$ de la matriz $\mathbf{A}^{t} \mathbf{A}, y$ $U R^{m \times m}$ es una matriz cuyas columnas son $A v_{i} / \sigma_{i} 0$ el vector nulo (en el caso de los $n-r$ valores singulares nulos). La ventaja de esta factorización, es que TODA matriz $A R^{m \times n}$ admite una descomposición por SVD. Puede probarse [22][23][24] que los métodos PCA y MCR obtienen la mejor estimación de rango $\mathrm{K}<\mathrm{r}$ para la matriz A.

El método MCR intenta recuperar perfiles de respuesta puros de las especies consituyentes (o componentes) de un sistema complejo, cuando no se tiene información disponible a priori sobre la naturaleza o composición del sistema. En general se aplica a datos en 2 dimensiones, aunque puede extenderse a dimensiones mayores. Básicamente, MCR propone que una matriz experimental $\mathbf{A}$ de rango $\mathrm{K}$ de $\mathrm{n}$ filas y $\mathrm{m}$ columnas puede expresarse como:

$$
\mathbf{A}=\mathbf{C S}^{\dagger}+\mathbf{E} \Leftrightarrow a_{i j}=\sum_{k=1}^{p} c_{i p} S_{p j}+e_{i j}
$$

Donde $\mathbf{C}$ es una matriz $n \times p, \mathbf{S}$ es $m \times p$ y $\mathbf{E}$ es $m \times n$. $\mathbf{C}$ y $\mathbf{S}$ representan las contribuciones en las dos dimensiones del experimento de los $p$ componentes y $\mathbf{E}$ es la matriz que recoge el error en la estimación. En principio, este método es similar al análisis de componentes principales (PCA, del inglés Principal Component Analysis), ya que ambos obtienen la mejor estimación de $\mathbf{A}$ en una matriz $\mathbf{C S}^{\mathrm{t}}$ de rango $\mathrm{k}<\mathrm{K}$ minimizando la siguiente expresión:

$$
\left\|\mathbf{A}-\mathbf{C S}^{\mathbf{t}}\right\|^{2}=\|\mathbf{E}\|^{2} \Leftrightarrow \sum_{i, j} e_{i j}^{2}=\sum_{i, j}\left(a_{i j}-\sum_{k=1}^{p} c_{i p} s_{p j}\right)^{2}
$$

Sin embargo, el método de PCA posee el inconveniente de que sus soluciones no son únicas (Se obtienen soluciones que no son invariantes por rotaciones ortogonales con una matriz $\mathbf{Q}$ que cumpla $\mathbf{Q} \mathbf{Q}=\mathbf{Q} \mathbf{Q}=\mathbf{I}$ siendo I de dimensiones $p \times p$ ), que en MCR es atacado agregando restricciones (no negatividad de los valores de $C$ o S, unimodalidad en los perfiles obtenidos, etc.) al sistema matricial. Las técnicas descriptas en 2.8 .2 son casos particulares de las enunciadas aquí, donde se utiliza como condición el comportamiento exponencial de cada componente en la dimensión temporal del experimento.

El método PARAFAC es una generalización de MCR a experimentos con datos en 3 dimensiones, donde se cambia una variable experimental extra además de lo descripto para el método MCR. Si tenemos un arreglo de datos ordenados en $n$ matrices $\mathrm{M}$, tal que el arreglo es $\mathbf{M} n \times \mathrm{m} \times \mathrm{s}$, PARAFAC expresa la estimación como: 


$$
\mathbf{M}_{\mathrm{n}}=\mathbf{B} \mathbf{a}_{\mathrm{n}} \mathbf{C}^{\mathrm{t}}+\mathbf{E}_{\mathrm{n}} \Leftrightarrow \mathrm{m}_{\mathrm{ijk}}=\sum_{r=1}^{p} b_{i r} a_{j r} c_{k r}+e_{i j k}
$$

Donde $B$ es una matriz $m \times p, e_{n}$ es una matriz $p \times p, C$ es $p \times s$ y $E_{n}$ es $m \times s$; y las sumas se realizan sobre los $p$ componentes. Los vectores $\mathbf{a}_{\mathbf{i}}, \mathbf{b}_{\mathbf{i}}$ y $\mathbf{c}_{\mathbf{i}}$ describen el comportamiento del i-ésimo componente con respecto a cada variable experimental. Entonces las matrices $A=\left[a_{1} \ldots a_{p}\right], B=\left[b_{1} \ldots b_{p}\right]$, y $C=\left[C_{1} \ldots C_{p}\right]$ indican las variaciones de los componentes en los distintos parámetros del experimento; y $\mathbf{E}$ es el arreglo $\mathrm{n} \times \mathrm{m} \times \mathrm{s}$ que recoge el error en la estimación. La ventaja de PARAFAC sobre MCR es que el uso de un modelo trilineal evita el problema rotacional que mencionamos en MCR y PCA.

Los datos experimentales usados para este tipo de análisis deben cumplir el requerimiento de linealidad entre los componentes del modelo propuesto. Usamos estos métodos de análisis para matrices de emisión excitación (EEM, del inglés Emission Excitation Matrix), considerando que para baja absorción del fluoróforo (A $<0.05)$, la intensidad de emisión en estado estacionario a longitud de onda de emisión y excitación dadas, $\mathrm{I}\left(\lambda_{\mathrm{em}}\right)$, es proporcional al coeficiente de absorción del fluoróforo a la longitud de onda de excitación , $\varepsilon\left(\lambda_{\mathrm{ex}}\right)$, y a un factor $\mathrm{F}\left(\lambda_{\mathrm{em}}\right)$ que refleja la probabilidad del proceso de emisión desde el estado excitado emisor a los distintos estados vibracionales del estado fundamental. Usando estas relaciones de linealidad, pueden aplicarse MCR o PARAFAC (en caso de EEMs obtenidas en distintas condiciones) para obtener información sobre el número mínimo de componentes y sus espectros de emisión y excitación en un sistema complejo. En casos en los cuales los espectros se superpongan, puede que los distintos espectros no lleguen a resolverse; y en el caso de componentes con contribuciones muy dispares (por ej. emisión dominante de un componente) el análisis multilineal puede llegar a no diferenciar estos componentes, sino entregar información sobre especies con propiedades ópticas muy relacionadas.

\subsubsection{Análisis global y Análisis objetivo}

La espectroscopía resuelta en el tiempo es una herramienta utilizada ampliamente para estudiar la dinámica de sistemas fisicoquímicos complejos. Esta puede emplearse para analizar desde la estructura de la superficie de energía potencial de un estado excitado hasta la interacción de un cromóforo con su entorno cercano vía transferencia de carga o energía. Ejemplos interesantes del uso de esta herramientas son los estudios de proteínas fotoactictivas [25], la bomba de transporte de protones bacteriorodopsina [26][27], o los fotosistemas I y |l del aparato fotosintético [5].

Al momento de entender la definición de las técnicas análisis global y objetivo, los trabajos del grupo de Beechem [28] son fundamentales. El análisis global implica el análisis estadístico simultáneo de una colección de trazas temporales, obtenidas detectando en distintas condiciones de observación (En estudios espectroscópicos suelen colectarse a distintas longitudes de onda de detección). En el caso de probar un modelo fotofísico o fotoquímico particular, la técnica se llama análisis objetivo.

En experimentos espectroscópicos resueltos en el tiempo, la muestra se excita usando un pulso intenso de RE que se absorbe y desencadena una serie de reacciones, las cuales vienen acompañadas por cambios en las propiedades espectroscópicas en el UV, visible o IR que pueden ser medidas. A su vez, la escala temporal de detección puede ir desde los femtosegundos hasta los segundos. Los datos adquiridos son una colección de medidas de propiedades ópticas, como intensidad de emisión o absorción, en función del tiempo y la longitud de onda de detección.

Los datos obtenidos en estos experimentos son ejemplos de datos en 2 dimensiones. Este tipo de arreglo de datos suelen armarse a partir de una variable independiente espectral (longitud de onda, número de onda, intensidad de campo magnético, etc), y una segunda variable experimental controlada de forma independiente (tiempo luego de la excitación, temperatura, polarización del haz incidente, pH, longitud de onda de excitación, 
concentración de quencher, etc). A su vez, la suma de una variable extra conduce a arreglos de 3 dimensiones, cuyo análisis se explica en la sección 2.8.1.

Para develar los procesos responsables de los cambios espectroscópicos observados, que se ven expresados en una cantidad importante de datos experimentales, es imperativo realizar un análisis basado en algún modelo. Desde esta perspectiva, pueden distinguirse 2 problemas: (a) Existe un modelo parametrizado para los observables medidos, y los parámetros se estiman usando procedimientos estadísticos contundentes o (b) Solo se conoce (o en principio se cree útil) un tipo de modelo, con lo cual el problema pasa también por el elegir el modelo más adecuado.

En principio, al momento de realizar un análisis de los resultados de experimentos espectroscópicos resueltos en el tiempo es necesario conocer (si es posible) el perfil temporal del de la respuesta del instrumento (conocido como IRF, del inglés Instrument Response Function) y el comportamiento estadístico del error en las medidas experimental.

Por un lado, el IRF es el producto de convolución del comportamiento temporal del pulso de excitación $\mathrm{E}(\mathrm{t})$ y la respuesta del detector $R(t)$,

$$
\operatorname{IRF}(t)=E(t) \otimes R(t)=\int E(s) R(t-s) d s
$$

Donde el intervalo de integración es el dominio de $E(t)$ y $R(t)$. Idealmente, debe estar por debajo de la escala de tiempo del proceso más rápido a estudiar en el sistema problema. En caso de que sean comparables, es sumamente necesario conocer y deconvolucionar el IRF de los datos al momento del análisis, y la precisión en la sincronía de la medida. Para estimarlo, se usan distintas técnicas, según el experimento a realizar: (i) Puede determinarse el IRF a $\lambda_{\text {exc }} y$ estimarlo a diferentes $\lambda$ usando un parámetro de corrimiento (típico en experimentos de emisión, utilizado en este trabajo) (ii) usar un compuesto con comportamiento temporal conocido, y obtenerlo por deconvolución o (iii) utilizar un modelo parametrizado. En el caso de experimentos pump/probe, el IRF viene dado por el producto de convolución del pulso de bombeo (pump) y de detección (probe). En estos experimentos, existe la complicación adicional de la dependencia del IRF con $\lambda$ de detección, originada en la variación de la velocidad de grupo con $\lambda$. Este efecto suele llamarse chirp, y puede corregirse estimando el corrimiento del IRF a partir de los datos a analizar, del solvente puro o a partir de medidas de correlación cruzada.

Con respecto al comportamiento aleatorio del error experimental, existen técnicas particulares como TCPSC donde el comportamiento es conocido (la probabilidad de detección sigue una distribución de Poisson y cada evento de detección es independiente), mientras que en otras suele asumirse que el error es aditivo, sigue una distribución normal y los eventos de detección son independientes. En el caso de detección con arreglos de diodos o cámaras CCD a tiempos controlados, donde el espectro se registra completo en un paso, uno no puede asegurar que los datos obtenidos para cada $\lambda$ sean independientes, lo cual puede ser incluido en el modelado del experimento con una matriz de covarianza $\boldsymbol{\Sigma}$. A su vez, es deseable que la varianza $\sigma^{2}$ de las medidas no dependa de $t$ ni de $\lambda$.

Para develar los procesos responsables de los cambios espectroscópicos observados, que se reflejan en una gran cantidad de datos experimentales, es imperativo realizar un análisis basado en algún modelo. Desde esta perspectiva, pueden distinguirse 2 problemas: (a) Existe un modelo parametrizado para los observables medidos, y los parámetros se estiman usando procedimientos estadísticos o (b) Solo se conoce (o en principio se cree útil) un tipo de modelo, con lo cual el problema pasa también por el elegir el modelo más adecuado. En este punto, el análisis global ofrece una vía para diferenciar modelos que ajustan de forma similar al comportamiento experimental registrado o para generar modelos útiles para un fenómeno en estudio. El análisis global analiza bajo el mismo modelo distintos experimentos, vinculando los parámetros obtenidos para probar la robustez del modelo propuesto. El objetivo de esta metodología es reunir la información de todo el conjunto 
de datos en el comportamiento cinético y espectral de pocos componentes. Si consideramos que $A(\lambda, t)$ es el espectro resuelto en el tiempo obtenido experimentalmente, este puede expresarse como:

$$
\mathrm{A}(\lambda, \mathrm{t})=\sum_{j=1}^{N} C_{j}(t) \varepsilon_{j}(\lambda)+e(t, \lambda)
$$

La suma se extiende sobre todos los $\mathrm{N}$ componentes presentes en el espectro, siendo $\mathrm{C}_{\mathrm{j}}(\mathrm{t})$ la evolución temporal y $\varepsilon_{j}(\lambda)$ el perfil espectral del j-ésimo componente, y e $(t, \lambda)$ es el error experimental.

Los modelos más básicos que uno puede imaginar para describir $A(\lambda, t)$ son modelos cinéticos de especies que decaen de formas simples:

- Secuencial $(1 \rightarrow 2 \rightarrow \ldots \rightarrow N)$, donde el decaimiento entre las especies (o compartimentos) j y j+1 es exponencial con constante $k_{j}$. Los espectros asociados son llamados EAS $(\lambda)$ (del inglés Evolution Asociated Spectra) cuyos tiempos de decaimiento son $t_{j}=1 / k_{j}$, y en general $k_{j}>k_{j+1}$ (o equivalentemente $\left.t_{j}<t_{j+1}\right)$. Por definición, el primer EAS corresponde al espectro al $t=0$ del experimento. En este modelo, a t $\sim 1 / \mathrm{k}_{\mathrm{j}}$ la especie dominante en el comportamiento espectral será j.

- Paralelo $(1,2, \ldots, N$ presentes a $t=0)$ donde las especies decaen simultáneamente de forma exponencial con constantes $k_{j}$. A diferencia del modelo secuencial, a $t \sim 1 / k_{j}$ la especie dominante no necesariamente es $\mathrm{j}$ y para el espectro $\mathrm{a} t=0$ no necesariamente coincide con el de algún componente resultante del análisis. En este modelo, a cada constante $\mathrm{k}_{\mathrm{j}}$ se asigna un espectro al cual se denomina $\operatorname{DAS}(\lambda)$ (del inglés Decay Asociated Spectra), que viene dado por la amplitud del decaimiento exponencial con constante $k_{j} a t=0$. Un modelo paralelo para $A(\lambda, t)$ propone

$$
\mathrm{A}(\lambda, \mathrm{t})=\mathrm{A}_{0}+\sum_{j} A_{j, 0}(\lambda) \mathrm{e}^{-k_{j} t}
$$

Si bien estos modelos parecen ser distintos, bajo ciertas condiciones son matemáticamente equivalentes [29][28], lo cual probaremos más adelante.

La aparente simpleza de estos modelos trae consigo suposiciones importantes:

- El problema es separable, esto es, las partes espectrales y temporales pueden describirse con funciones que solo dependan de una variable. Debido al efecto del chirp, en la escala de los fs la independencia de estas partes resulta comprometida, lo cual debe tener en cuenta al momento de la estimación de constantes en esta escala de tiempos.

- El sistema de estudio presenta una sola población, tal que las constantes determinadas representan al sistema completo. En el caso de que existan distintas poblaciones dentro del sistema estudiado, pueden utilizarse promedios pesados para la descripción de las constantes o bien distribuciones de constantes de velocidad. Estos sistemas inhomogéneos son comunes en nanotecnología, y el entendimiento de los procesos involucrados no es una tarea trivial.

Una forma de generalizar los posibles modelos, es considerar $\mathrm{N}$ compartimientos que se comunican entre sí por constantes de transferencia $\mathrm{k}_{\mathrm{j}}$. Estos compartimientos pueden representar estados o un conjunto de estados energéticamente cercanos, tal que puedan actuar como un conjunto con una constante única de transferencia ${ }^{4}$ a los fines del modelo. Si los procesos cinéticos presentes en el modelo son de primer orden, este puede escribirse como un sistema de ecuaciones diferenciales lineales:

${ }^{4}$ Este tipo de suposiciones se realizan en casos de estados cuyas constantes de transferencia son muy cercanas comparadas a la inversa de la mínima resolución temporal en el experimento, tal que no puedan ser correctamente resueltas. 


$$
\frac{d}{d t} \mathbf{C}(t)=K C(t)+j(t)
$$

Donde el vector $\mathbf{C}(\mathrm{t})=\left[\mathbf{C}_{1}(\mathrm{t}) \ldots \mathbf{C}_{\mathrm{N}}(\mathrm{t})\right]^{\mathrm{t}}$ contiene la evolución temporal de los $\mathrm{N}$ compartimentos, $\mathrm{j}(\mathrm{t})=\operatorname{IRF}(\mathrm{t})\left[\mathrm{x}_{1} \ldots\right.$ $\left.x_{N}\right] t$ representa el pulso de excitación que inicia la cascada de procesos siendo $x_{j}$ el parámetro que corresponde al efecto sobre el j-ésimo compartimento, y finalmente la matriz $\mathrm{K}$ que contiene las constantes de transferencia $\mathrm{k}_{\mathrm{ij}}$ para los procesos que comunican los compartimentos $\mathrm{i}$ con $\mathrm{j}$.

Si se establece como condición de borde que $C(-\infty)=0$, lo cual es lógico ya que para tiempos menores a $t=0$ el pulso de excitación no interactúa con la muestra, la ecuación (2.18) tiene solución analítica:

$$
\mathbf{C}(\mathrm{t})=\exp (\mathbf{K} t) \otimes \mathbf{j}(\mathrm{t})
$$

En el caso de que $\mathbf{j}(t)=\operatorname{IRF}(t)[1 \ldots 1]$ y que la matriz $\mathbf{K}$ sea diagonal $\mathbf{K}=\operatorname{diag}\left(-\mathrm{k}_{1}, \ldots,-\mathrm{k}_{\mathrm{N}}\right)$, el vector $\mathbf{C}^{\mathrm{p}}(\mathrm{t})$ tiene elementos $C_{j}^{p}=\exp \left(-k_{j} t\right) \otimes I R F(t)$. Como puede verse, este caso representa al modelo de decaimientos paralelos.

En el caso de que la matriz $\mathbf{K}$ no sea diagonal, puede emplearse la descomposición en valores y vectores propios tal que $\mathbf{K}=\mathbf{U k U}^{-1}$, siendo $\mathbf{U}$ la matriz de autovectores de $\mathbf{K}$ y $\mathbf{k}$ la matriz diagonal de autovalores de $\mathbf{K}$, $\mathbf{k}=\operatorname{diag}\left(-\mathrm{k}_{1}, \ldots,-\mathrm{k}_{\mathrm{N}}\right)$. La expresión para $\mathrm{C}(\mathrm{t})$ resulta:

$$
\begin{gathered}
\mathbf{C}(\mathrm{t})=\exp (\mathbf{K} t)=\mathbf{U} e^{\mathbf{k} t} \mathbf{U}^{-1} \\
\mathbf{C}(\mathrm{t})=\exp (\mathbf{K} t) \otimes \mathbf{j}(\mathrm{t})=\mathbf{U} \operatorname{diag}\left(\mathbf{U}^{-1}\left[x_{1} x_{2} \cdots x_{N}\right]^{t}\right)\left[e^{-k_{1} t} \otimes I R F(t) \cdots e^{-k_{N} t} \otimes I R F(t)\right]^{t}
\end{gathered}
$$

Puede verse que, para el modelo general, cada elemento de $\mathbf{C}(\mathrm{t})$ es una combinación lineal de los elementos de $\mathbf{C p}(\mathrm{t})$.

En el caso del modelo secuencial la matriz K es una matriz triangular inferior, siempre y cuando no se consideren reacciones reversibles. La expresión para el k-ésimo elemento del vector $\mathbf{C}(\mathrm{t})$ resulta:

$$
\begin{aligned}
& \mathrm{C}_{\mathrm{k}}(\mathrm{t})=\sum_{j=1}^{k} \mathrm{~b}_{\mathrm{j}} \mathrm{e}\left(-\mathrm{k}_{\mathrm{j}} t\right) \otimes I R F(\mathrm{t}) \\
& \mathrm{b}_{\mathrm{j} k}=\prod_{m=1}^{l-1} k_{m}
\end{aligned}
$$

Donde los coeficientes $b_{\mathrm{jk}}$ son las amplitudes convolucionadas de cada término.

En el caso de considerar reacciones reversibles (o inhomogeneidades en el estado fundamental, como se comentó previamente) el comportamiento es multiexponencial. Por cada compartimiento involucrado, hay una exponencial extra, si consideramos el siguiente ejemplo (donde $P$ es el único estado emisor) $Q \leftrightarrow R \leftrightarrow P \rightarrow h v$ el decaimiento será triexponencial. A su vez, como $P$ es el único emisor, un sistema vinculado de forma similar, por ejemplo $R \leftrightarrow P \leftrightarrow Q$, no podría diferenciarse del anterior usando experimentos de emisión resueltos en el tiempo. A su vez, es posible que debido a la no emisión o absorción en la ventana espectral del experimento haya compartimientos los cuales no pueden ser detectados, generando transiciones silenciosas, lo cual conduce a una interpretación errónea del fenómeno físico en estudio. Por estas razones, es de fundamental importancia atacar al mismo problema con diversas técnicas experimentales. Para una discusión más detallada 
sobre modelos para análisis de experimentos ópticos resueltos en el tiempo, puede consultarse los trabajos del grupo de Van Grondelle [29], [30].

A su vez, para obtener espectros que puedan asociarse a especies individuales (SAS, del inglés Species Asociated Spectra) es necesario obtener en primer lugar, información experimental extra que permita diferenciar comportamientos particulares dentro del modelo propuesto. Esta información puede buscarse en experimentos con luz polarizada, en medios de distinta naturaleza que alteren la estructura de la superficie de energía potencial de los estados excitados involucrados, etc. Luego de esto, pueden refinarse los modelos de análisis empleados, usando mecanismos particulares propuestos por el experimentalista. Este refinamiento es llamado análisis objetivo, o también target analysis en inglés.

Para estos análisis se usaron el software Glotaran [31], desarrollado en R (licencia GPL) y los paquetes ASUfit y ultrafast [18] desarrollados en Matlab ${ }^{\circledR}$.

\subsection{Detección de especies reactivas de oxígeno}

\subsubsection{Detección por fosforescencia de oxígeno singlete $\left(\mathrm{O}_{2}\left(\mathrm{a}^{1} \Delta \mathrm{g}\right)\right)$}

La presencia del oxígeno singlete $\left(\mathrm{O}_{2}\left(\mathrm{a}^{1} \Delta \mathrm{g}\right)\right)$ se determinó mediante la detección de su fosforescencia a 1273 nm debido a la relajación radiativa desde el estado singlete de menor energía al estado triplete basal del oxígeno molecular. Para ello se utilizaron suspensiones saturadas en oxígeno del sistema de interés, las cuales excitamos con distintos armónicos de un láser Nd:YAG Surelite II Continuum laser (7 ns FWHM y 14 mJ/pulso @ $355 \mathrm{~nm}$ ). La radiación emitida se detectó en ángulo recto usando un amplificador Judson J 116/8sp y un detector de germanio (5 $\mathrm{mm}$ de diámetro) después de pasar por los filtros apropiados. Las medidas se compararon con las señales obtenidas a partir de compuestos estándar para la sensibilización de $\mathrm{O}_{2}\left(\mathrm{a}^{1} \Delta \mathrm{g}\right)$, los cuales fueron elegidos según la longitud de onda de excitación y el solvente de la muestra problema (A menos que se indique lo contrario, usamos 5,10,15,20-Tetrafenil-21H,23H-porfirina, TPP, para excitación a $355 \mathrm{~nm}$ y 3,4,5,6-tetracloro-2',4',5',7'-tetraiodo-fluoresceína, dianión, Rosa de Bengala RB, para excitación a 532 nm). Los decaimientos se promediaron y almacenaron en un osciloscopio Agilent Infiniium $500 \mathrm{MHz}$. Las medidas se realizaron irradiando a $355 \mathrm{~nm} 0532 \mathrm{~nm}$. Los solventes utilizados fueron tolueno, acetonitrilo y agua deuterada $\left(\mathrm{D}_{2} \mathrm{O}\right)$ con un pD de 6,4 calculado añadiendo 0,4 a los valores de pH medidos (Martire et al. 1993).

\subsubsection{Detección de $\mathrm{O}_{2}\left(\mathrm{a}^{1} \Delta \mathrm{g}\right)$ mediante un Electrodo Sensible de $\mathrm{O}_{2}$ molecular}

La formación de $\mathrm{O}_{2}\left(\mathrm{a}^{1} \Delta \mathrm{g}\right)$ por las nanopartículas de silicio como sensibilizadores se detectó de forma indirecta mediante el monitoreo de consumo de oxígeno molecular en un reactor cerrado. El montaje experimental consta de una lámpara Rayonet irradiando a una longitud de onda de $(350 \pm 20) \mathrm{nm}$ en un reactor cerrado donde se sitúa el fotosensibilizador, un electrodo sensible de oxígeno molecular (Orion 97-0899) y un compuesto capaz de reaccionar física o químicamente con el $\mathrm{O}_{2}\left(\mathrm{a}^{1} \Delta \mathrm{g}\right)$ en suspensiones saturadas en aire.

La técnica se basa en medir el consumo de oxígeno triplete molecular $\left(\mathrm{O}_{2}\left({ }^{3} \Sigma \mathrm{g}\right)\right)$ en función del tiempo de irradiación en un reactor cerrado mediante un electrodo sensible a la concentración de $\mathrm{O}_{2}\left({ }^{3} \Sigma \mathrm{g}\right)$. Para tal propósito se realizan tres experiencias diferentes. En el primer experimento se irradian suspensiones acuosas de Si nps y se mide el consumo de oxígeno. Si se registra algún consumo de oxígeno podría ser debido a que el oxígeno singlete generado estuviese reaccionando con la superficie de las propias Si nps. De lo contrario, la energía de excitación electrónica del oxígeno singlete es eficientemente convertida en energía vibracional al desactivarse por las moléculas de solvente según la reacción 2.30, volviendo al estado basal del oxígeno y no consumiéndose. En el segundo experimento, se añade alcohol furfurílico (FA) a la suspensión anterior. Este reactivo es conocido por su eficacia en reaccionar con oxígeno singlete siguiendo la reacción (2.31). Como consecuencia de esta reacción se elimina $\mathrm{O}_{2}\left({ }^{3} \Sigma \mathrm{g}\right)$ del medio, siendo detectado este consumo por el electrodo. La tercera experiencia se basa en añadir azida sódica $\left(\mathrm{NaN}_{3}\right)$ a la anterior solución en una concentración un 
orden de magnitud mayor que la de FA. El oxígeno singlete interacciona con la azida sódica siguiendo el esquema de reacción (2.32), desactivándose al estado basal del oxígeno por lo que este no se consume. Al existir una competencia por el $\mathrm{O}_{2}\left(\mathrm{a}^{1} \Delta \mathrm{g}\right)$ entre el alcohol furfurílico y la azida sódica, donde la $\mathrm{NaN}_{3}$ se encuentra en mayor proporción, se inhibe el consumo de $\mathrm{O}_{2}\left(\mathrm{a}^{1} \Delta \mathrm{g}\right)$ tal como se espera por la reacción 2.32.

$$
\begin{array}{ll}
\mathrm{O}_{2}\left(\mathrm{a}^{1} \Delta_{\mathrm{g}}\right) \rightarrow \mathrm{O}_{2}\left({ }^{1} \Sigma_{\mathrm{g}}\right) & \mathrm{k} 1=2.9^{*} 10^{5} \mathrm{~s}^{-1} \\
\mathrm{O}_{2}\left(\mathrm{a}^{1} \Delta_{\mathrm{g}}\right)+\mathrm{FA} \rightarrow P & \mathrm{k} 2=1.2^{*} 10^{8} \mathrm{~s}^{-1} \mathrm{M}^{-1} \\
\mathrm{O}_{2}\left(\mathrm{a}^{1} \Delta_{\mathrm{g}}\right)+\mathrm{N}_{3}^{-} \rightarrow \mathrm{O}_{2}\left({ }^{1} \Sigma_{\mathrm{g}}\right)+\mathrm{N}_{3}^{-} & \mathrm{k} 1=4.5^{*} 10^{8} \mathrm{~s}^{-1} \mathrm{M}^{-1}
\end{array}
$$

\subsubsection{Detección de $\mathrm{O}_{2}\left(\mathrm{a}^{1} \Delta \mathrm{g}\right)$ mediante la sonda $\mathrm{SOSG} 囚$}

Un método alternativo para la detección de $\mathrm{O}_{2}\left(\mathrm{a}^{1} \Delta \mathrm{g}\right)$, es el uso de la sonda fluorescente conocida como "Singlet Oxygen Sensor Green" (SOSG®) que ha sido ampliamente utilizada en el estudio de materiales con aplicaciones médicas, por ej., en terapia fotodinámica (Ragàs et al. 2009). La sonda SOSG® se compone de un donante de electrones (derivado de antraceno) y un aceptor (derivado de fluoresceína) unidos covalentemente (Kim et al. 2013). SOSG® exhibe débiles picos de fluorescencia azul a 395 y $416 \mathrm{~nm}$ bajo excitación a 372 y $393 \mathrm{~nm}$. En presencia de $\mathrm{O}_{2}\left(\mathrm{a}^{1} \Delta \mathrm{g}\right)$, SOSG® emite fluorescencia verde con máximos de excitación y emisión a 504 y $525 \mathrm{~nm}$, respectivamente. La emisión de esta fluorescencia verde corresponde a la formación de un endoperóxido generado por la interacción del $\mathrm{O}_{2}\left(\mathrm{a}^{1} \Delta \mathrm{g}\right)$ con el residuo de antraceno del SOSG, como se observa para otras sondas similares de fluoresceína-antraceno.

Algunos estudios utilizando la sonda SOSG $®$ han demostrado la aparición de fluorescencia verde después de la exposición de $S O S G \circledast$ a radiación UV o visible, incluso en ausencia de un fotosensibilizador de $\mathrm{O}_{2}\left(\mathrm{a}^{1} \Delta \mathrm{g}\right)$ (Hideg 2008). También se sabe de la literatura, que la fluorescencia verde se desvanece bajo irradiación UV como consecuencia del proceso de fotoblanqueo mediado por radicales (Ragàs et al. 2009). Estos resultados deben tenerse en cuenta al utilizar la sonda diseñando el experimento con los controles adecuados.

\subsection{Materiales de Laboratorio}

\subsubsection{Limpieza del material}

Todo el material de vidrio empleado para síntesis o experimentos se limpió de forma cuidadosa para evitar la contaminación de la muestra, según el uso previo del material. A continuación, se comentan los procedimientos empleados. Luego del tratamiento empleado, el material se enjuagó profusamente usando agua destilada 0 ultra pura, según el destino del material.

\section{Mezcla sulfocrómica}

Se empleó mezcla sulfocrómica para remover contaminantes orgánicos de todo el material de vidrio previo a su uso en síntesis, debido a su alto poder oxidante. Se preparó disolviendo $5 \mathrm{~g}$ de K2Cr2O7 en $100 \mathrm{ml}$ de ácido sulfúrico concentrado (>95\%) y se utilizó hasta que su coloración se tornó verdosa. Esta solución se empleó como estándar de limpieza salvo casos especiales, en los cuales se necesitó tratamientos más agresivos o se limpió material óptico.

\section{Solución "piraña"}

La solución "piraña" se utilizó para lavar el material de vidrio y ciertos sustratos metálicos, particularmente los empleados para XPS. Es una solución fuertemente oxidante, compuesta por $\mathrm{H} 2 \mathrm{SO} 4$ concentrado y H2O2 100 vol en una relación volumétrica de $\mathrm{H} 2 \mathrm{SO} 4: \mathrm{H} 2 \mathrm{O} 2$ de 3:1. Se prepara agregando lentamente el ácido sobre el 
peróxido en baño de hielo de ser posible. Esta solución ataca violentamente los materiales orgánicos, por lo que se debe manipular con extrema precaución.

Agua regia

El material que ha estado en contacto con metales o sustancias orgánicas fue lavado con agua regia, que consiste en una mezcla de $\mathrm{HCl}$ y $\mathrm{HNO}_{3}$ concentrados en una relación volumétrica de $\mathrm{HCl}: \mathrm{HNO}_{3}=3: 1$. Se preparó momentos antes de ser utilizada y se manipuló bajo campana de extracción de gases. Esta solución disuelve los metales que se encuentren adheridos al material y ataca la materia orgánica. Se empleó tanto para el material de síntesis de Au nps y limpieza de material de cuarzo empleado en experimentos ópticos.

Potasa alcohólica

La potasa alcohólica se utilizó en casos de contaminantes orgánicos poco reactivos frente a mezcla sulfocrómica o en presencia de grasas. Esta mezcla se preparó disolviendo $20 \mathrm{~g}$ de $\mathrm{KOH}$ en $100 \mathrm{ml}$ de etanol comercial $(96 \%)$. Los materiales a limpiar se mantuvieron $\mathrm{x}$ un día en la mezcla y posteriormente fueron lavados con $\mathrm{HNO}_{3}$ diluido.

\subsubsection{Reactivos generales}

En este trabajo se utilizaron los solventes que se describen en la Tabla 3.1. Se procedió a reducir su contenido en agua mediante el uso de tamices moleculares de tamaño de malla $4 \AA$ (Anedra). Al momento de realizar medidas espectroscópicas, la calidad de los solventes empleados fue HPLC o superior. En general, se empleó agua destilada para la limpieza de material y la utilizada tanto en síntesis como en preparación de muestras fue de calidad ultra pura (Milli-Q尺 Millipore): Finalmente, presenta una resistividad mayor a $18 \mathrm{M} \Omega \mathrm{cm}$ a $25 \mathrm{C}$ y menos de 20 ppb de carbono orgánico.

\begin{tabular}{cccc}
\hline Reactivo & Fórmula & Pureza (\%) & Marca \\
\hline Ciclohexano & $\mathrm{C}_{6} \mathrm{H}_{12}$ & $>99$ & U.V.E \\
Etanol (EtOH) & $\mathrm{C}_{2} \mathrm{H}_{5} \mathrm{OH}$ & 99.9 & J.T. Baker \\
Metanol (MeOH) & $\mathrm{CH}_{3} \mathrm{OH}$ & 99.9 & J.T. Baker \\
Tolueno & $\mathrm{C}_{7} \mathrm{H}_{8}$ & 99.7 & J.T. Baker \\
N,N-Dimetilformamida (DMF) & $\left(\mathrm{CH}_{3}\right)_{2} \mathrm{NCOH}$ & $>99$ & Anedra \\
& & $>99.9$ & Sigma Aldrich \\
Acetonitrilo (MeCN) & $\mathrm{CH}_{3} \mathrm{CN}$ & 99.9 & J.T. Baker \\
& & $>99.9$ & Sigma Aldrich \\
Dimetilsulfóxido(DMSO) & $\left(\mathrm{CH}_{3}\right)_{2} \mathrm{SO}$ & $>99$ & Sintorgan \\
& & $>99.9$ & Sigma Aldrich \\
Óxido de deuterio & $\mathrm{D}_{2} \mathrm{O}$ & $99.9 \mathrm{D}$ atómico & Sigma Aldrich \\
Dietil éter & $\left(\mathrm{C}_{2} \mathrm{H}_{5}\right)_{2} \mathrm{O}$ & 98 & Cicarelli \\
$\mathrm{N}, \mathrm{N}$-dimetilacetamida (DMA) & $\left(\mathrm{CH}_{3}\right)_{2} \mathrm{NCOCH}_{3}$ & 99 & Sigma Aldrich \\
& Tabla 2.2. Solventes. & &
\end{tabular}

Los reactivos empleados en síntesis y protocolos experimentales fueron usados tal como fueron recibidos sin purificación posterior. En aquellos casos que fue necesario algún tratamiento extra, estos son mencionados y 
detallados oportunamente. Los reactivos utilizados a lo largo de este trabajo, junto con la calidad de los mismos, se detalla a continuación.

\begin{tabular}{|c|c|c|c|}
\hline Reactivo & Fórmula & Pureza (\%) & Marca \\
\hline Cloruro férrico & $\mathrm{FeCl}_{3} \cdot 6 \mathrm{H}_{2} \mathrm{O}$ & $>97$ & J.T. Baker \\
\hline Oxalato de potasio & $\mathrm{K}_{2}\left(\mathrm{CO}_{2}\right)_{2} \cdot \mathrm{H}_{2} \mathrm{O}$ & $>98.5$ & Anedra \\
\hline o-Fenantrolina & $\mathrm{C}_{12} \mathrm{H}_{8} \mathrm{~N}_{2} \cdot \mathrm{H}_{2} \mathrm{O}$ & 98 & Anedra \\
\hline Ácido nítrico & $\mathrm{HNO}_{3}$ & 65 & Anedra \\
\hline Ácido sulfúrico & $\mathrm{H}_{2} \mathrm{SO}_{4}$ & $95-98$ & Cicarelli \\
\hline Ácido clorhídrico & $\mathrm{HCl}$ & 37 & Merck \\
\hline Ácido fluorhídrico & $\mathrm{HF}$ & 50 & Cicarelli \\
\hline Peróxido de hidrógeno & $\mathrm{H}_{2} \mathrm{O}_{2}$ & 30 & Anedra \\
\hline Hidróxido de sodio & $\mathrm{NaOH}$ & 98.2 & J.T. Baker \\
\hline Hidrógeno fosfato de potasio & $\mathrm{K}_{2} \mathrm{HPO}_{4}$ & $>99$ & Sigma Aldrich \\
\hline Dihdrógeno fosfato de potasio & $\mathrm{KH}_{2} \mathrm{PO}_{4}$ & $>99$ & Sigma Aldrich \\
\hline Cloruro de silicio & $\mathrm{SiCl}_{4}$ & 99 & Sigma Aldrich \\
\hline $\begin{array}{l}\text { Bromuro de tetraoctil-amonio } \\
\text { (TOABr) }\end{array}$ & $\mathrm{N}\left(\mathrm{C}_{8} \mathrm{H}_{17}\right)_{4} \mathrm{Br}$ & 98 & Sigma Aldrich \\
\hline Tetrahidruro de aluminio y litio & $\mathrm{LiAlH}_{4}$ & 95 & Sigma Aldrich \\
\hline Tetrahidruro de boro y sodio & $\mathrm{NaBH} 4$ & 98 & Sigma Aldrich \\
\hline Formaldehído & $\mathrm{CH}_{2} \mathrm{O}$ & & Biopack \\
\hline 2-metil 2-propenoato de metilo & $\mathrm{C}_{4} \mathrm{H}_{8} \mathrm{O}_{2}$ & & \\
\hline 2-propenil amina & $\mathrm{C}_{3} \mathrm{H}_{5} \mathrm{NH}_{2}$ & 95 & Sigma Aldrich \\
\hline $\begin{array}{c}\text { tetraetoxisilano (TEOS) } \\
\text { 3-aminopropil-trietoxisilano }\end{array}$ & $\left(\mathrm{OC}_{2} \mathrm{H}_{5}\right)_{4} \mathrm{Si}$ & $>99$ & Merck \\
\hline (APTES) & $\mathrm{C}_{9} \mathrm{H}_{23} \mathrm{NO}_{3} \mathrm{Si}$ & $\geq 98$ & Sigma Aldrich \\
\hline $\begin{array}{c}\text { Amoniaco (solución acuosa) } \\
\text { Hexacloroplatinato de }\end{array}$ & $\mathrm{NH}_{3}$ & $26-30$ & Biopack \\
\hline hidrógeno & $\mathrm{H}_{2} \mathrm{PtCl}_{6} \times \mathrm{H}_{2} \mathrm{O}$ & 99.9 & Sigma Aldrich \\
\hline Tetracloroaurato de hidrógeno & $\mathrm{HAuCl}_{4} \cdot \mathrm{H}_{2} \mathrm{O}$ & 99.9 & Alfa Aesar \\
\hline hidroxilamina (Clorohidrato) & $\mathrm{NH}_{2} \mathrm{OH} \cdot \mathrm{HCl}$ & 99.9 & Sigma Aldrich \\
\hline \multirow[t]{2}{*}{ Citrato de sodio } & $\mathrm{C}_{6} \mathrm{H}_{5} \mathrm{Na}_{3} \mathrm{O}_{7} \cdot 2 \mathrm{H}_{2} \mathrm{O}$ & 98.5 & Cicarelli \\
\hline & & $>99$ & Sigma Aldrich \\
\hline Hidróxido de potasio & $\mathrm{KOH}$ & 85 & Anedra \\
\hline Siliciuro de magnesio & $\mathrm{Mg}_{2} \mathrm{Si}$ & $\geq 95$ & Sigma Aldrich \\
\hline Cloruro de amonio & $\mathrm{NH}_{4} \mathrm{Cl}$ & 99.5 & Carlo Erba \\
\hline Silicio (tipo p) & $\mathrm{Si}$ & $>99.9999$ & \\
\hline Sodio & $\mathrm{Na}$ & & \\
\hline Azida de sodio & $\mathrm{NaN}_{3}$ & & Sigma Aldrich \\
\hline Alcohol furfurílico & $\mathrm{C}_{5} \mathrm{H}_{6} \mathrm{O}_{2}$ & 99 & Sigma Aldrich \\
\hline
\end{tabular}




\begin{tabular}{|c|c|c|c|}
\hline Diciclohexilcarbodiimida (DCC) & $\mathrm{C}_{13} \mathrm{H}_{22} \mathrm{~N}_{2}$ & 99 & Sigma Aldrich \\
\hline N-hidroxisuccinimida (NHS) & $\mathrm{C}_{4} \mathrm{H}_{5} \mathrm{NO}_{3}$ & 98 & Sigma Aldrich \\
\hline $\begin{array}{c}\text { Cloruro de olaxlilo } \\
\text { Hexafluorofosfato de fluoro- } \\
\text { N,N,N',N'- } \\
\text { tetrametilformamidinio (TFFH) }\end{array}$ & {$\left[\mathrm{FC}\left[\mathrm{N}\left(\mathrm{CH}_{3}\right)_{2}\right] \mathrm{N}\left(\mathrm{CH}_{3}\right)_{2}\right] \mathrm{PF}_{6}$} & 98 & Sigma Aldrich \\
\hline $\begin{array}{c}\text { 9, 10- difenil antraceno } \\
(9,10-\text { DPA }) \\
\text { 3',6'-dihidroxiespiro }\end{array}$ & $\mathrm{C}_{26} \mathrm{H}_{18}$ & 98 & Fluka \\
\hline $\begin{array}{l}\text { [2-benzofuran-3,9'-xanten]-1- } \\
\text { ona dianión (Fluoresceína) }\end{array}$ & $\mathrm{C}_{20} \mathrm{H}_{10} \mathrm{O}_{5} \mathrm{Na}_{2}$ & $>98.5$ & Fluka \\
\hline $\begin{array}{l}\text { Isotiocianato de fluoresceína } \\
\text { (FITC) } \\
\text { 3,4,5,6-tetracloro-2',4',5',7'- } \\
\text { tetraiodo-fluoresceína, dianión }\end{array}$ & $\mathrm{C}_{21} \mathrm{H}_{11} \mathrm{NO}_{5} \mathrm{~S}$ & 90 & Sigma Aldrich \\
\hline Antraceno & $\begin{array}{c}\mathrm{C}_{14} \mathrm{H}_{10} \\
\mathrm{C}_{20} \mathrm{H}_{24} \mathrm{~N}_{2} \mathrm{O}_{2} \cdot 1 / 2\end{array}$ & $>96$ & Merck \\
\hline Sulfato de quinina & $\mathrm{H}_{2} \mathrm{SO}_{4} \cdot \mathrm{H}_{2} \mathrm{O}$ & 99.7 & Sigma Aldrich \\
\hline $\begin{array}{c}5,10,15,20 \text {-Tetrafenil-21H,23H- } \\
\text { porfirina (TPP) }\end{array}$ & $\mathrm{C}_{44} \mathrm{H}_{30} \mathrm{~N}_{4}$ & $>99$ & Sigma Aldrich \\
\hline Triptófano & $\mathrm{C}_{11} \mathrm{H}_{12} \mathrm{~N}_{2} \mathrm{O}_{2}$ & $>98$ & Sigma Aldrich \\
\hline
\end{tabular}

Tabla 2.3. Reactivos utilizados.

Los gases utilizados fueron: Argón (Calidad 4 bandas) y Oxígeno suministrados por La Oxígena S.A., Argentina. Previo a su uso, se los hizo circular por una trampa de nitrógeno líquido para eliminar la humedad residual.

\subsubsection{Equipamiento menor}

Las pesadas se realizaron en una balanza electrónica marca Denver modelo TP-214 de $\pm 0,1 \mathrm{mg}$ de precisión.

Se utilizó un equipo de ultrasonidos marca TESLAB S.R.L. Modelo TB04TA con una potencia de 160 W y una frecuencia de $40 \mathrm{kHz}$.

En los procesos de aislamiento y purificación se emplearon filtros de membrana de Nylon con poros de 0.22 $\mu \mathrm{m}$ (Osmonics inc.) y membranas de diálisis de $20.4 \mathrm{~mm}$ de diámetro con corte de pesos moleculares de 6000 - 8000 (Spectra/por@).

Los cambios de solvente se llevaron a cabo en un evaporador rotatorio Senco. En caso de ser necesario, se trabajó a presión reducida acoplando el sistema a una bomba mecánica de vacío, y los gases evacuados fueron atrapados con una trampa de nitrógeno líquido.

Las medidas de $\mathrm{pH}$ se hicieron con un pHmetro modelo Consort $\mathrm{C} 832$ y las medidas de $\mathrm{O} 2$ disuelto con un electrodo selectivo de $\mathrm{O} 2$ multicanal modelo Consort C932 unido a una PC.

En la vía electroquímica de síntesis la intensidad de corriente aplicada se reguló con un potenciostato LYP M6 con señal triangular con control de corriente de $\pm 0,01 \mathrm{~mA}$. A su vez, para monitorear la corriente que circulaba por el circuito se conectó un multímetro digital en serie. 


\subsection{Referencias}

[1] J. R. Lakowicz, Principles of fluorescence spectroscopy. 2006.

[2] C. F. Landes, S. Link, M. B. Mohamed, B. Nikoobakht, and M. a. El-Sayed, "Some properties of spherical and rod-shaped semiconductor and metal nanocrystals," Pure Appl. Chem., vol. 74, no. 9, pp. 1675-1692, 2002.

[3] S. N. Timasheff, "TURBIDITY AS A CRITERION OF COAGULATION In the study of aggregating or coagulating macromolecular systems, the I = K' $\mathrm{Nr} 2(\mathrm{r} / \mathrm{X}) \sim=\mathrm{KI}$ ' I ( ) V = XVo ;", vol. 7, 1966.

[4] A. J. Bard, L. R. Faulkner, N. York, C. @bullet, W. Brisbane, and S. E. Toronto, ELECTROCHEMICAL METHODS Fundamentals and Applications. 1944.

[5] J. D. Megiatto, A. Antoniuk-Pablant, B. D. Sherman, G. Kodis, M. Gervaldo, T. A. Moore, A. L. Moore, and D. Gust, "Mimicking the electron transfer chain in photosystem II with a molecular triad thermodynamically capable of water oxidation," Proc. Natl. Acad. Sci., vol. 109, no. 39, pp. 1557815583, 2012.

[6] B. Valeur, Molecular Fluorescence: Principles and applications, vol. 8. 2001.

[7] D. Gust, T. A. Moore, and A. L. Moore, "Mimicking photosynthetic solar energy transduction," Acc. Chem. Res., vol. 34, no. 1, pp. 40-48, 2001.

[8] P. V. Kamat, "Meeting the clean energy demand: Nanoestructure Architectures for Solar Energy Conversion," Phys. Chem., vol. 392, pp. 2834-2860, 2007.

[9] S. Farid, J. P. Dinnocenzo, P. B. Merkel, R. H. Young, D. Shukla, and G. Guirado, "Reexamination of the Rehm À Weller Data Set Reveals Electron Transfer Quenching That Follows a Sandros À Boltzmann Dependence on Free Energy," vol. 0, no. Figure 1, pp. 11580-11587, 2011.

[10] R. A. Marcus, "Electrostatic Free Energy and Other Properties of States Having $\mathrm{N}$ onequilibrium Polarization. 1*," vol. 24, no. 5, pp. 10-13, 1956.

[11] R. A. Marcus, "ON T H E T H E O R Y O F ELECTROCHEMICAL AND CHEMICAL ELECTRON," vol. 37, no. 2, 1968.

[12] R. A. Marcus, "On the Theory of Oxidation-Reduction Reactions Involving Electron Transfer. 1*," vol. 42, no. 1952, pp. 966-978, 1956.

[13] K. Ohkubo, H. Imahori, J. Shao, Z. Ou, K. M. Kadish, Y. Chen, G. Zheng, R. K. Pandey, M. Fujitsuka, and O. Ito, "Small Reorganization Energy of Intramolecular Electron Transfer in Fullerene-Based Dyads with Short Linkage," pp. 10991-10998, 2002.

[14] I. R. Could, J. E. Maser, and S. Farid, "Efficiencies of Photoinduced Electron-Transfer Reactions: Role of the Marcus Inverted Region in Return Electron Transfer within Geminate Radical-Ion Pairs," no. d, pp. 4290-4301, 1990.

[15] M. M. Waskasi, G. Kodis, A. L. Moore, T. A. Moore, and D. V Matyushov, "Marcus Bell-Shaped Electron Transfer Kinetics Observed in an Arrhenius Plot," no. 2, 2016.

[16] A. Mikhailovsky, "Basics of femtosecond laser spectroscopy What's so Special About Femtosecond Lasers ???," no. 805. 
[17] A. . Fallis, Principles of Lasers, vol. 53, no. 9. 2013.

[18] L. J. G. W. van Wilderen, C. N. Lincoln, and J. J. van Thor, "Modelling multi-pulse population dynamics from ultrafast spectroscopy," PLoS One, vol. 6, no. 3, 2011.

[19] I. Sullivan, C. L. Brown, M. J. Llansola-Portoles, M. Gervaldo, G. Kodis, T. A. Moore, D. Gust, A. L. Moore, and P. A. Maggard, "Photoinjection of High Potential Holes into Cu<inf $>5</$ inf $>$ Ta $<$ inf $>11</$ inf $>0<$ inf $>30</$ inf $>$ Nanoparticles by Porphyrin Dyes," J. Phys. Chem. C, vol. 119, no. 37, pp. 21294-21303, 2015.

[20] A. Antoniuk-Pablant, G. Kodis, A. L. Moore, T. A. Moore, and D. Gust, "Photoinduced electron and energy transfer in a molecular triad featuring a fullerene redox mediator," In Print, 2015.

[21] J. Shlens, J. Shlens, a We, and a We, "A Tutorial on Principal Component Analysis," Measurement, 2005.

[22] M. Interuniversitario, D. A. Ucm, T. Análisis, O. Pca, and A. Pca, "Análisis de datos y Estadística Avanzada Esquema," 2011.

[23] A. Stegeman, "The Parafac Model for Multi-way Data Analysis," pp. 1-52.

[24] R. Bro, "PARAFAC. Tutorial and applications," Chemom. Intell. Lab. Syst., vol. 38, no. 2, pp. 149-171, 1997.

[25] T. E. Meyer, E. Yakali, M. a Cusanovich, and G. Tollin, "Properties of a water-soluble, yellow protein isolated from a halophilic phototrophic bacterium that has photochemical activity analogous to sensory rhodopsin.," Biochemistry, vol. 26, no. 2, pp. 418-23, 1987.

[26] R. H. Lozier, R. A. Bogomolni, and W. Stoeckenius, "Bacteriorhodopsin: a light-driven proton pump in Halobacterium Halobium," Biophys. J., vol. 15, no. 9, pp. 955-962, 1975.

[27] J. Wang and M. a El-Sayed, "Temperature jump-induced secondary structural change of the membrane protein bacteriorhodopsin in the premelting temperature region: a nanosecond timeresolved Fourier transform infrared study.," Biophys. J., vol. 76, no. 5, pp. 2777-2783, 1999.

[28] J. M. Beechem, M. Ameloot, and L. Brand, "Global and Target Analysis of Complex Decay Phenomena," Instrum. Sci. Technol., vol. 14, no. 3-4, pp. 379-402, 1985.

[29] I. H. M. Van Stokkum, D. S. Larsen, and R. Van Grondelle, "Global and target analysis of timeresolved spectra," Biochim. Biophys. Acta - Bioenerg., vol. 1657, no. 2-3, pp. 82-104, 2004.

[30] I. H. M. Van Stokkum, D. S. Larsen, R. Van Grondelle, I. H. M. van Stokkum, R. van Grondelle, D. S. Larsen, and R. van Grondelle, "Erratum to 'Global and target analysis of time-resolved spectra' [Biochimica et Biophysica Acta 1658/2-3 (2004) 82-104]," Biochim. Biophys. Acta - Bioenerg., vol. 1658, no. 3, p. 262, 2004.

[31] J. J. Snellenburg, S. P. Laptenok, R. Seger, K. M. Mullen, and I. H. M. van Stokkum, "Glotaran : A Java -Based Graphical User Interface for the R Package TIMP," J. Stat. Softw., vol. 49, no. 3, p. , 2012. 
- 64 - 


\section{Capítulo 3. Aspectos Sintéticos}

En el presente capítulo se discuten los detalles experimentales relacionados a la síntesis y purificación de los distintos bloques de construcción empleados en este trabajo, los cuales fueron Si nps, Au nps y colorantes. Luego se consideran las estrategias usadas para conjugar estos bloques y obtener los ensamblados estudiados a lo largo de esta tesis.

\subsection{Síntesis de Si nps}

A lo largo de este trabajo se emplearon diversos métodos para la síntesis de Si nps. Se utilizaron estrategias tanto de "arriba hacia abajo" (TD, del inglés top-down) como de "abajo hacia arriba" (BU, dei inglés bottom-up), utilizando como criterio de aceptación la calidad de la distribución de tamaños y el control sobre las propiedades superficiales. La caracterización y propiedades fotofísicas de las Si nps obtenidas se discute en detalle en los capítulos 4 y 5 .

\subsubsection{Síntesis de Si nps de $<d>=(3 \pm 1)$ nm por grabado electroquímico (TD’ Si nps)}

Esta técnica es una adaptación realizada por Llansola [1] de la publicada por Sailor y colaboradores [2]. En este procedimiento se anodizó un cristal de $\mathrm{Si}<100>$ tipo $\mathrm{p}$ con resistividad $\rho=3.5-7 \Omega \mathrm{cm}$ en presencia de $\mathrm{HF}$ en condiciones de densidad de corriente (j) y potencial (E) controlados.

Para obtener buenos resultados en esta técnica es de suma importancia controlar el valor de j, ya que manteniendo constantes la composición del electrolito soporte y el tipo de cristal de Si, este parámetro determina el mecanismo operativo en la electrodisolución del Si. Dicho comportamiento se muestra en la figura 3.1. El mecanismo de electrodisolución de Si en presencia de HF y diversos aditivos ha sido estudiado en detalle en literatura [3] [4]. Básicamente, este procede de dos regímenes bien diferenciados:

- A bajas j, el proceso inicia por un paso netamente electródico donde se intercambian 2 Faraday de carga por mol de $\mathrm{Si}$, generando una serie de complejos [ $\mathrm{SiF}_{2}$ ] en la superficie del electrodo (3.1). Luego estos complejos $\left[\mathrm{SiF}_{2}\right]$ son atacados por los aniones $\mathrm{F}^{-}$presentes y en presencia de $\mathrm{H}^{+}$se observa el desprendimiento $\mathrm{H}_{2(g)}$ y $\mathrm{SiF}_{6}$ - a la solución (3.2). El proceso neto puede verse como una oxidación que requiere el intercambio de 2 Faraday a través del electrodo (3.3.). Este régimen se denomina de formación de Si poroso (pSi), y es de fundamental importancia en las aplicaciones nanotecnológicas del Si. Si consideramos como equivalentes oxidantes los huecos en la banda de valencia del $\mathrm{Si}\left(\mathrm{h}^{+}\right)$, el mecanismo de formación de pSi puede entenderse considerado las siguientes reacciones.

$$
\begin{aligned}
& \mathrm{Si}+2 \mathrm{~h}^{+}+2 \mathrm{~F}^{-} \rightarrow\left[\mathrm{SiF}_{2}\right] \\
& {\left[\mathrm{SiF}_{2}\right]+4 \mathrm{~F}^{-}+2 \mathrm{H}^{+} \rightarrow\left[\mathrm{SiF}_{6}\right]^{2-}+\mathrm{H}_{2}} \\
& \mathrm{Si}+2 \mathrm{~h}^{+}+6 \mathrm{~F}^{-}+2 \mathrm{H}^{+} \rightarrow\left[\mathrm{SiF}_{6}\right]^{2-}+\mathrm{H}_{2}
\end{aligned}
$$

Este proceso cuenta con el detalle de ser direccionado e influenciado por las facetas cirstalinas expuestas por el cristal de Si, lo cual genera el crecimiento orientado de poros y permite la disposición espacial precisa de regiones de porosidad controlada.

- A altas j, el proceso es completamente electródico, intercambiándose 4 Faradays por mol de Si (3.4). Este proceso es isotrópico, logrando la remoción de capas atómicas de Si. Con este proceso, el blanco de Si se vuelve más delgado y la superficie es pulida. Por esta razón, este régimen es conocido como 
electropulido o electropolishing, en inglés. Este proceso puede utilizarse para remover de forma controlada capas porosas generadas en pasos previos de electrólisis.

$$
\mathrm{Si}+4 \mathrm{~h}^{+}+6 \mathrm{~F}^{-} \rightarrow\left[\mathrm{SiF}_{6}\right]^{2-}
$$

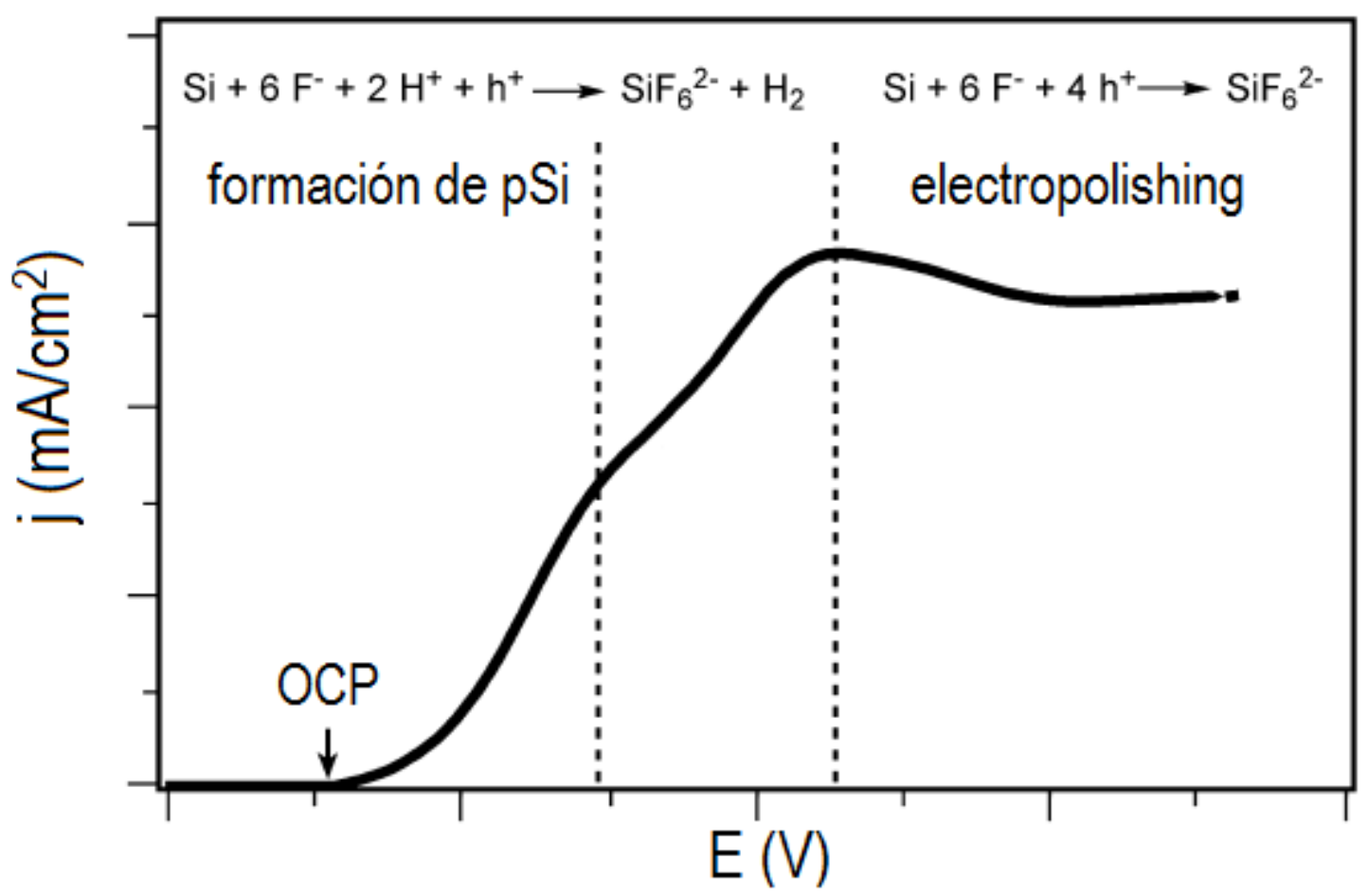

Figura 3.1. Comportamiento electroquímico del Si en fase masiva. Se pueden diferenciar el potencial de circuito abierto (Open Circuit Potential, en inglés) y las regiones de formación de pSi y electropolishing. Adaptado de [2].

Al observar los procesos involucrados en la electrodisolución del Si, queda en evidencia el rol preponderante que toman j y la composición del electrolito soporte. En condiciones tales que el mecanismo operativo sea el de generación de $\mathrm{pSi}$, la composición del electrolito soporte determina la terminación de la superficie luego de la reacción. La relación entre el flujo de carga hacia la superficie del electrodo (dado por j) y el flujo difusivo de iones F- determinará si las moléculas de agua pueden actuar como el nucleófilo de la reacción (3.2). Si el caso fuese este último, se formaría una capa pasiva de $\mathrm{SiO}_{2}$ que aisla el electrodo y produce finalmente el desprendimiento de la capa porosa, por reacción con HF. Esta competencia se esquematiza en la figura 3.2. 


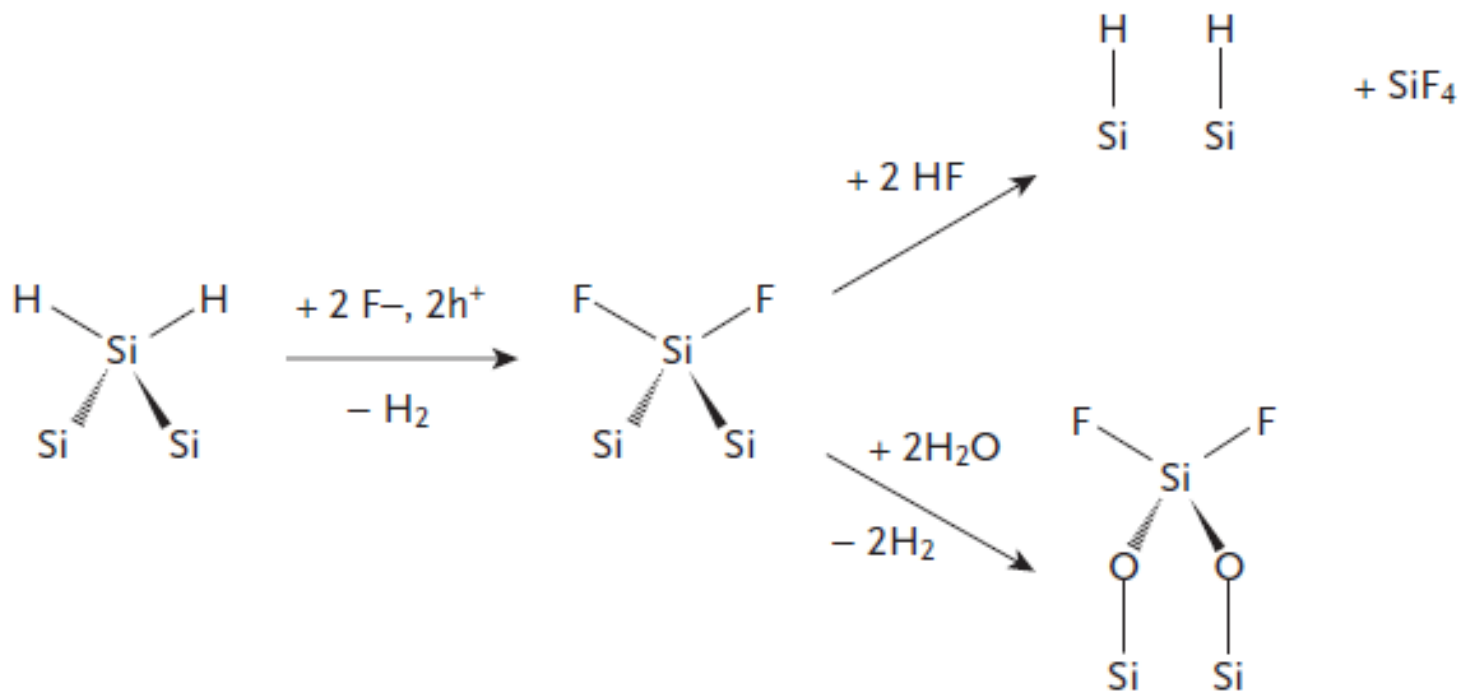

Figura 3.2. Procesos en competencia correspondientes a la formación de $\mathrm{pSi}$. En la rama superior se esquematiza la reacción en cantidades suficientes de HF, mientras que en la rama inferior se muestra la reacción en condiciones de HF en defecto, permitiendo la participación de moléculas de agua en la reacción de adición nucleofilica. Adaptado de [2].

Con respecto al procedimiento experimental, se tomó una pieza de Si de aproximadamente $20 \times 20 \mathrm{~mm}^{2}$ que fue pulida mecánicamente, primero en presencia de $\mathrm{MeOH}$ y luego utilizando una solución de $\mathrm{HF} 30 \% \mathrm{v} / \mathrm{v}$ en $\mathrm{MeOH}$, a fin de quitar la capa polimérica de protección y la capa pasivante de $\mathrm{SiO}_{2}$ que están presentes en las obleas de Si empleadas. Este paso es de suma importancia para garantizar el control sobre la terminación de la superficie, permitiendo que esta presente hidrógenos terminales. Luego, el electrodo de trabajo fue colocado en una celda de teflon conteniendo $30.00 \mathrm{ml}$ de solución de electrolito soporte (16\% HF, $16 \% \mathrm{MeOH}$ y $68 \%$ agua ultrapura). Como contraelectrodo se empleó un anillo de Pt, para optimizar la distribución de líneas de corriente. Es de suma importancia corroborar la buena conexión de los terminales del potenciostato/galvanostato con los electrodos, particularmente con el ánodo de $\mathrm{Si}$, a fin de evitar caídas óhmicas inesperadas que afecten las condiciones de densidad de corriente empleadas. Como medida de control, se conectó en serie a la celda electroquímica un multímetro para monitorear la corriente que circulaba por la celda.

La electrólisis se realizó en condiciones de corriente constante i= $20.0 \mathrm{~mA}\left(\mathrm{j}=5 \mathrm{~mA} / \mathrm{cm}^{2}\right.$ aproximadamente) el mayor tiempo posible, sumergiendo el electrodo de trabajo en la solución a $5 \mathrm{~mm} / \mathrm{h}$, a fin de renovar la superficie expuesta en el proceso de rugosado. Luego de la electrólisis, el cristal de Si se enjuagó con EtOH y se transfirió a un baño de tolueno en atmósfera inerte de Ar. Este sistema se colocó en un baño de ultrasonidos por 2 horas y el material desprendido fue filtrado usando filtros hidrofóbicos de PVDF (Millex-HV, Millipore) de $220 \mathrm{~nm}$ de tamaño de poro. Luego de esto, se colectaron y almacenaron las 2 fases: La fase orgánica que consiste en una dispersión de Si nps de $\langle\mathrm{d}\rangle=(3 \pm 1) \mathrm{nm}$, y el sólido filtrado que es $\mathrm{pSi}$ desprendido del electrodo. Este último se secó bajo corriente de Ar y almacenó para su posterior uso. Las Si nps de $\langle d\rangle=(3 \pm 1) \mathrm{nm}$ obtenidas en dispersión en este proceso se denominaron TD' Si nps. Cabe resaltar que estás partículas se encuentran recubiertas por una capa de óxidos de silicio.

\subsubsection{Sintesis de Si nps de $<d>=(1.7 \pm 0.3) \mathrm{nm}$ por disolución con HF (TD Si nps)}

Se utilizó un ataque químico con HF para reducir el tamaño de partículas micrométricas de pSi obtenidas en 3.2.1. Este proceso aprovecha la disolución química del $\mathrm{SiO}_{2}$ usando $\mathrm{HF}$, liberando $\mathrm{Si}$ nps ocluidas en la matriz porosa de $\mathrm{Si} / \mathrm{SiO}_{2}$. Teniendo esto en cuenta, sería posible controlar el tamaño de partícula obtenido al regular el tiempo de reacción empleado [5]. El éxito de esta estrategia depende fuertemente de que la distribución de 
tamaños de las partículas de pSi obtenidas previamente sea lo suficientemente estrecha, tal que la disolución de partículas muy pequeñas y la ruptura de grandes aglomerados sean procesos competitivos que permitan sintonizar el tamaño de las nps obtenidas.

En un vaso de precipitados plástico se colocaron $3.0 \mathrm{mg}$ de pSi obtenidos en 3.2 .1 y se dispersaron en 12.00 $\mathrm{ml}$ de una solución 3\% v/v de HF en $\mathrm{MeOH}$. Esta suspensión se colocó en un baño de ultrasonidos hasta observar la dispersión del sólido inicial, lo cual demoraba alrededor de 30 minutos. Posteriormente, se retiró del baño y se almacenó en oscuridad durante $20 \mathrm{hs}$. Pasado este período se agregaron $20.00 \mathrm{ml}$ de agua ultrapura a la suspensión y se trasvasó a una ampolla de decantación. Las Si nps hidrofóbicas dispersas fueron extraídas empleando 4 extracciones con $5.00 \mathrm{ml}$ de ciclohexano. En cada paso de extracción se verificó la presencia/ausencia de Si nps en fase acuosa irradiando la suspensión con una lámpara de $350 \mathrm{~nm}$ y observando a ojo desnudo la emisión de las Si nps. Finalmente, se colectó la fase orgánica en su totalidad y se calentó a $60 \mathrm{C}$ durante 9 hs. Finalmente, se evaporó el solvente a presión reducida y las Si nps fueron dispersadas en tolueno para su posterior uso. Las nps obtenidas por esta técnica presentaron un $\langle d\rangle=(1.7 \pm$ 0.3) nm y se denominaron TD Si nps.

\subsubsection{Síntesis de Si nps de $<d>=(1.7 \pm 0.8)$ nm por reducción en micelas inversas (BU Si nps)}

Esta vía de síntesis es una adaptación de la publicada por Rosso Vasic et al. [6]. Para controlar de forma eficiente el tamaño de las Si nps sintetizadas, una opción viable es el empleo de micelas de algún surfactante adecuado como nano-reactores. En general, se emplean compuestos del tipo $\mathrm{SiX}_{4}(\mathrm{X}=\mathrm{Cl}, \mathrm{Br}$ o I), que se localizan en el interior hidrofilico de la micela, que por el agregado de un reductor originan Si nps cuyo crecimiento está restringido por el entorno micelar. El tamaño de las nps puede controlarse limitando el tamaño de las micelas, interacciones intermicelares, variando la temperatura o la relación precursor de Si / reductor.

En un balón de reacción, se colocaron $16.50 \mathrm{ml}$ de tolueno y $6.3 \mathrm{~g}$ de TOABr. Esta dispersión se colocó en un baño de ultrasonidos durante 20 minutos bajo atmósfera inerte de $\mathrm{Ar}$ a temperatura ambiente $(\mathrm{T}=20 \mathrm{C})$; manteniendo un burbujeo constante durante toda la síntesis. En este punto, la mezcla se torna amarillenta. Posteriormente se colocan $0.63 \mathrm{ml}$ de $\mathrm{SiCl}_{4}$ y se continúa con la agitación durante 20 minutos más, para favorecer el ingreso del reactivo a las micelas. Aquí la mezcla tomó un color blanquecino. Una vez pasado el tiempo, se agregan $10.00 \mathrm{ml}$ de solución $1 \mathrm{M}$ de $\mathrm{LiAlH}_{4}$ en éter etílico y se agita por 30 minutos. Luego de este paso es conveniente verificar la presencia de nps, tomando una alícuota e iluminándola con luz de $350 \mathrm{~nm}$, para observar a ojo desnudo la emisión por parte de las Si nps. Esta alícuota debe contener la menor cantidad de surfactante posible, ya que este desactiva la luminiscencia de las NP. Una vez verificada la presencia de nps, se agregó a la mezcla $30.00 \mathrm{ml}$ de $\mathrm{MeOH}$ para inhibir el reductor. Se mantuvo en agitación la mezcla durante 30 minutos más. Para aislar las Si nps generadas, la mezcla se filtró y se evaporó el solvente empleando un evaporador rotatorio. Luego de esto, el sólido remanente se redisolvió en $30.00 \mathrm{ml}$ de ciclohexano, dejando separar las fases. La fase líquida se filtró y evaporó nuevamente. Para extraer el producto final adherido a las paredes del balón se agregaron $25.00 \mathrm{ml}$ de tolueno.

En la figura 3.3 se esquematiza la vía de síntesis detallada. Las reacciones involucradas en el proceso son las siguientes:

$$
\begin{aligned}
& \mathrm{SiCl}_{4}+\mathrm{LiAlH}_{4} \rightarrow \mathrm{SiH}_{4}+\mathrm{LiCl}+\mathrm{AlCl}_{3} \\
& \mathrm{SiH}_{4}+\mathrm{SiCl}_{4} \rightarrow \mathrm{H}_{3} \mathrm{SiSiCl}_{3}+\mathrm{HCl} \\
& \mathrm{SiH}_{4}+\mathrm{SiH}_{4} \rightarrow \mathrm{Si}_{2} \mathrm{H}_{6}+\mathrm{H}_{2} \\
& \mathrm{SiH}_{4}+\mathrm{H}_{3} \mathrm{SiSiC}_{3} \rightarrow \mathrm{SiH}_{3} \mathrm{SiH}_{2} \mathrm{SiCl}_{3}+\mathrm{HCl}
\end{aligned}
$$

Siendo (3.2), (3.3), (3.4) y reacciones similares a esta, pasos fundamentales en el crecimiento de las np. Para estas Si nps se determinó $<\mathrm{d}>=(1.7 \pm 0.8) \mathrm{nm}$, y fueron denominadas BU Si nps. 


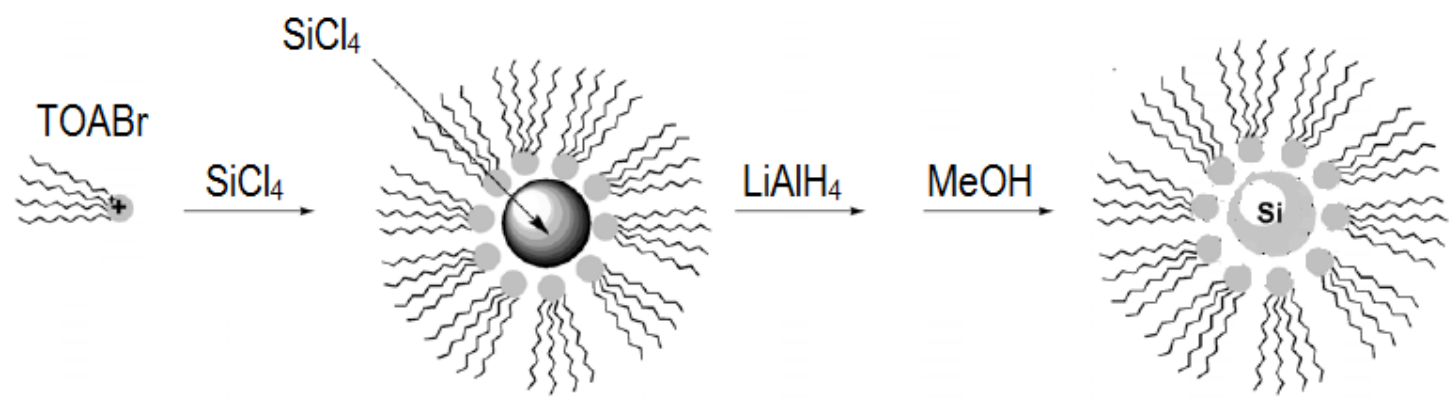

Figura 3.3. Síntesis de BU Si nps por reducción de precursores de Si en presencia de micelas invertidas. Adaptado de [6].

\subsubsection{Sintesis de Si nps de $<d>=(3.4 \pm 0.6)$ nm por oxidación en fase líquida (BU' Si nps - S1)}

Esta estrategia está basada en los trabajos publicados por Kauzlarich y colaboradores [7][8][9]. En esta vía, se emplearon reacciones de oxidación de fases de Zintl donde el Si sea un constituyente. En bibliografía se ha explorado el uso tanto de distintas fases de Zintl como de oxidantes de distinta fuerza. Una vez elegido el par redox a emplear, la variación de temperatura a lo largo de la reacción o la relación de precursor de Si a oxidante $\left(n_{S_{i}}: n_{o x}\right)$ puede emplearse para controlar el tamaño de las Si nps obtenidas. Si bien este tipo de técnicas son muy versátiles y permiten no solo la obtención de Si nps, sino que también puede extenderse a nps de elementos del grupo IV e incluso nanopartículas dopadas, las muestras son polidispersas si la reacción no se controla lo suficiente. En este trabajo de tesis se utilizó como par redox $\mathrm{Mg}_{2} \mathrm{Si}$ y $\mathrm{NH}_{4} \mathrm{Cl}$, y la reacción empleada fue la siguiente:

$$
\mathrm{Mg}_{2} \mathrm{Si}+4 \mathrm{NH}_{4} \mathrm{Cl} \rightarrow 4 \mathrm{NH}_{3}+2 \mathrm{H}_{2}+2 \mathrm{MgCl}_{2}+\mathrm{Si}
$$

En un balón con 3 bocas se colocaron $120.00 \mathrm{ml}$ de DMF seco y 100.0 mg de $\mathrm{Mg}_{2} \mathrm{Si}$ ( $1.3 \mathrm{mmol}$ ) en agitación. Esta solución se calienta hasta $100 \mathrm{C}$ y se purga con Ar por 30 minutos. Posteriormente se agregaron 200.0 $\mathrm{mg}$ de $\mathrm{NH}_{4} \mathrm{Cl}\left(3.5 \mathrm{mmol}\right.$, $\left.\mathrm{n}_{\mathrm{si}}: \mathrm{n}_{\mathrm{ox}} \sim 1: 3\right)$ y se continuó el calentamiento y la purga, hasta que la mezcla entró en reflujo ( $153 \mathrm{C}$ ), luego de lo cual se cortó el burbujeo con Ar. En este punto, la mezcla se ve de color levemente azul, debido a partículas de $\mathrm{Mg}_{2} \mathrm{Si}$ en suspensión. La reacción procedió por 20 hs, y luego se enfrió lentamente. El avance de la reacción puede verificarse tomando una alícuota e iluminándola con luz de $350 \mathrm{~nm}$, para observar a ojo desnudo la emisión por parte de las Si nps. Al finalizar la reacción, la mezcla posee un color anaranjado. Esta mezcla se filtró usando membranas de $220 \mathrm{~nm}$ para remover el $\mathrm{Mg}_{2} \mathrm{Si}$ que no reaccionó, y luego se dializó contra solvente puro para remover los productos secundarios.

\subsubsection{Sintesis de Si nps de $<d>=(4 \pm 1)$ nm por oxidación en fase líquida (BU' Si nps - S4)}

Esta síntesis se realizó siguiendo el mismo protocolo que 3.2.4. Las cantidades empleadas fueron $120.00 \mathrm{ml}$ de DMF, $100.0 \mathrm{mg}$ de $\left(1.3 \mathrm{mmol}\right.$ ) y $800.0 \mathrm{mg}$ de $\mathrm{NH}_{4} \mathrm{Cl}\left(3.5 \mathrm{mmol}, \mathrm{n}_{\mathrm{si}}: \mathrm{n}_{\mathrm{ox}} \sim 1: 5\right)$. El aislamiento y la purificación se realizó del modo detallado en la sección 3.2.4.

\subsubsection{Sintesis de Si nps de $<d>=(5 \pm 1) n m$ por oxidación en fase líquida (BU' Si nps - S3)}

Esta síntesis se realizó siguiendo el mismo protocolo que 3.2.4. Las cantidades empleadas fueron $120.00 \mathrm{ml}$ de DMF, $100.0 \mathrm{mg}$ de (1.3 mmol) y $400.0 \mathrm{mg}$ de $\mathrm{NH}_{4} \mathrm{Cl}$ (3.5 mmol, $\left.\mathrm{n}_{\mathrm{si}}: \mathrm{n}_{\mathrm{ox}} \sim 1: 11\right)$. El aislamiento y la purificación se realizó del modo detallado en la sección 3.2.4.

\subsubsection{Sintesis de Si nps de $<d>=(3.2 \pm 0.5)$ nm por oxidación en fase líquida (BU' Si nps $-S C$ )}

Esta síntesis se realizó siguiendo el mismo protocolo que 3.2.4. modificando el precursor de Si. En este caso usamos $\mathrm{NaSi}$, la reacción es la siguiente: 


$$
2 \mathrm{NaSi}+2 \mathrm{NH}_{4} \mathrm{Cl} \rightarrow 2 \mathrm{NH}_{3}+\mathrm{H}_{2}+2 \mathrm{NaCl}+2 \mathrm{Si}
$$

Las cantidades empleadas fueron $120.00 \mathrm{ml}$ de DMF, $100.0 \mathrm{mg}$ de NaSi (1.96 mmol) y $600.0 \mathrm{mg} \mathrm{de} \mathrm{NH}_{4} \mathrm{Cl}$ (3.5 $\left.\mathrm{mmol}, \mathrm{n}_{\mathrm{si}}: \mathrm{n}_{\mathrm{ox}} \sim 1: 2\right)$. El aislamiento y la purificación se realizó del modo detallado en la sección 3.2.4.

\subsubsection{Determinación de la concentración de Si nps}

Para realizar ensayos en los cuales es necesario conocer la concentración analítica de Si nps, esta fue determinada usando espectrometría de emisión atómica (ICP - AES), la distribución de tamaños obtenida por TEM, y la densidad del Si en fase masiva $\left(\delta_{\mathrm{Si}}=2.33 \mathrm{~g} / \mathrm{cm}^{3}\right)$. Usando $<\mathrm{d}>$ obtenido por TEM y $\delta_{\mathrm{Si}}$ se estimó la masa molecular promedio de las Si nps $\left\langle P M_{\text {Si np }}>=\delta_{\mathrm{Si}}(1 / 6) \pi<\mathrm{d}\right\rangle^{3}$. A su vez, los resultados de ICP - AES fueron determinados como ppm (mg/l) de Si. Por lo tanto, usando los resultados de ICP - AES junto a la estimación de $\left\langle P M_{\text {si np }}>\right.$, pudieron hacerse estimaciones de la concentración molar de Si nps.

\subsection{Control superficial}

Generalmente, los objetivos de la modificación superficial implican proporcionar cierta afinidad al sustrato tal que permitan obtener suspensiones estables del mismo o generar posibilidades de reacción que la superficie original no proporciona, permitiendo así funcionalizaciones con moléculas de diverso interés.

Los métodos convencionales para funcionalizar superficies de Si involucran la formación de enlaces Si-C o Si$\mathrm{O}-\mathrm{C}$, cuya estabilidad es lo suficientemente alta para proteger la superficie de futuras oxidaciones. La oxidación de la superficie de Si en nanopartículas juega un rol muy importante, como se discute en el capítulo 4, por lo cual debe evitarse o mantenerse controlada. Las superficies limpias de Si presentan pasivación de $\mathrm{H}$, y si bien el enlace Si-H es lo suficientemente estable $\left(\Delta \mathrm{H}_{\mathrm{Si}-\mathrm{H}^{0}}=95 \mathrm{kcal} / \mathrm{mol}\right.$, estimado a partir del compuesto $\left.\left(\mathrm{CH}_{3}\right)_{3} \mathrm{SiH}\right)$, en presencia de agua la oxidación del Si es termodinámicamente favorable, por lo cual las Si nps recién sintetizadas expuestas a la atmósfera comienzan a oxidarse de poco a poco. Entonces, para evitar el inconveniente de oxidaciones indeseadas empleamos reacciones de modificación superficial. Existen diversas reacciones que llevan a la formación de estos enlaces, sin embargo las que ofrecen mayores ventajas son las reacciones de hidrosililación y silanización.

Al momento de estimar las cantidades necesarias de reactivos para llevar adelante las reacciones de modificación superficial, se utilizó a modo de guía la estructura de la superficie de Si considerando una reconstrucción $2 \times 1$ en superficies (100), cuya densidad de $\mathrm{H}$ superficiales es de $12 \mathrm{H} / \mathrm{nm}^{2}$, y se ajustaron las cantidades empleadas a condiciones de exceso de 1.1 equivalentes del compuesto a unir en superficie por cada 1 equivalente de $\mathrm{H}$ en superficie.

\subsubsection{Reacciones de hidrosililación}

En la hidrosililación, se adiciona $\mathrm{Si}$ y $\mathrm{H}$ sobre un enlace $\mathrm{C}-\mathrm{C}$ insaturado. A su vez, los átomos de $\mathrm{Cl}$ o $\mathrm{Br}$ superficiales también permiten derivatizar la superficie mediante reacciones similares. Estas reacciones pueden ser llevadas adelante empleando alquenos terminales e iniciadas por luz [10] o calor [11], o empleando catalizadores de Pt [12]. La gran ventaja de estas reacciones es que no se rompen enlaces Si-Si, lo cual es de fundamental importancia en Si nps pequeñas. De hecho, las rutas que involucren el clivaje de dichos enlaces pueden ocasionar variaciones en las propiedades ópticas, y en el caso de partículas muy pequeñas hasta la disolución completa [11]. Esta desventaja la presentan las reacciones de alcoxilación, donde pueden formarse enlaces Si-H o Si-O-C, y las alquilaciones por reactivos de Grignard o alquil-litios, donde la reacción procede por un ataque nucleofilico del carbanión sobre un átomo de Si con una baja densidad electrónica.

Las reacciones de sililación pueden proceder por diversos mecanismos, según sea el paso de iniciación. En este trabajo de tesis se empleó generalmente la vía fotoquímica salvo casos aislados, en el cual se usó la vía térmica catalizada por $\mathrm{H}_{2} \mathrm{PtCl}_{6}$. El mecanismo por el cual procede esta reacción depende de diversos factores, 
y en el caso de la estratégica fotoquímica es importante el rol de la longitud de onda de la radiación empleada. La elección correcta de la vía a emplear permite minimizar la aparición de productos indeseados que posteriormente complican tanto la purificación de las muestras como el análisis de resultados [13]. En las condiciones de síntesis empleadas para la vía fotoquímica, el mecanismo propuesto se esquematiza en la figura 3.3. En el caso de la vía térmica se cree que el mecanismo catalizado consta de un ataque electrofílico sobre el enlace insaturado del compuesto insaturado, el cual se ve favorecido por la orientación particular y la coordinación del complejo de Pt sobre el compuesto no saturado [6].
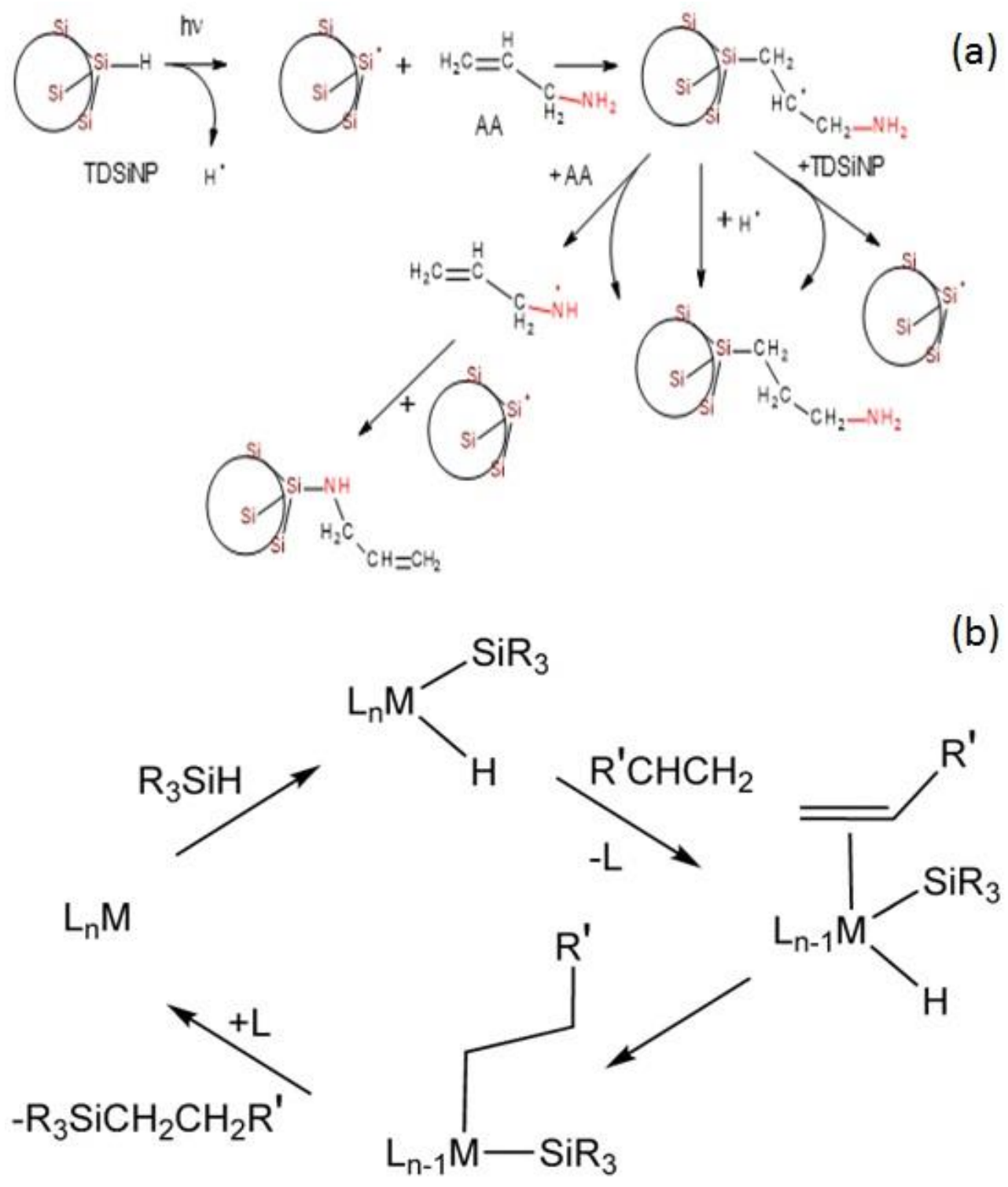

Figura 3.3. Mecanismo propuesto para la reacción de sililación (a) iniciada por vía fotoquímica con radiación de UV o (b) por vía térmica catalizada con complejos metálicos. En este trabajo usamos ambas técnicas.

Para minimizar efectos indeseados, se estandarizó un protocolo de modificación post-sintética, para reacciones de sililación tanto por vía térmica como fotoquímica.

En el primer caso, se colocó en un balón de 3 bocas que contenía las cantidades adecuadas de Si nps en suspensión en DMF y del precursor orgánico según se indicó en 3.3 y $500 \mu \mathrm{l}$ de una solución de $\mathrm{H}_{2} \mathrm{PtCl}_{6} 5 \mathrm{mM}$ en $\mathrm{MeOH}$, completando con DMF hasta llegar a volumen 120.00 ml. Luego la mezcla de reacción se calentó 
lentamente hasta llegar a reflujo. Simultáneamente se purga la mezcla profusamente con Ar hasta el inicio del reflujo. La reacción se llevó a cabo durante aproximadamente 20 hs, para luego retirar el calentamiento y enfriar lentamente. Finalmente, las Si nps fueron aisladas y purificadas por diálisis y ciclos de evaporación/disolución en el solvente de trabajo.

En el segundo caso, se mezclaron las cantidades requeridas de Si nps en suspensión de tolueno (o DMF en casos particulares) y compuestos orgánicos en un reactor cilíndrico de cuarzo, completando con solvente hasta $40.00 \mathrm{ml}$. Esta mezcla se purgó con Ar durante 30 minutos y posteriormente fue sellada con parafilm $®$. Finalmente, Este sistema fue colocado en irradiación en un fotorreactor con agitación constante con 8 lámparas de $254 \mathrm{~nm}$ por $5 \mathrm{hs}$ y finalmente fue retirado del mismo. Finalmente, las Si nps fueron aisladas y purificadas por diálisis y ciclos de evaporación/disolución en el solvente de trabajo.

\subsubsection{Reacciones de silanización}

En las reacciones de silanización se aprovecha la estabilidad de los puentes siloxano (Si-O-Si) y la versatilidad de los procesos de hidrólisis/condensación. El uso de este tipo de reacciones en superficies extendidas de Si es inmediato, debido a la presencia de una capa pasivante de $\mathrm{SiO}_{2}$. En general, la superficie a tratar se expone a cantidades controladas de agua y moléculas del silano a acoplar en un solvente adecuado[14].

En el caso de $\mathrm{Si}$ ns, la capa de $\mathrm{SiO}_{2}$ se forma espontáneamente en suspensiones equilibradas con aire, si las Si nps no son protegidas con ligandos orgánicos luego de sintetizadas. Por lo tanto, el uso de esta reacción para modificar superficies de Si nps podría constituir una alternativa a la hidrosililación[15].
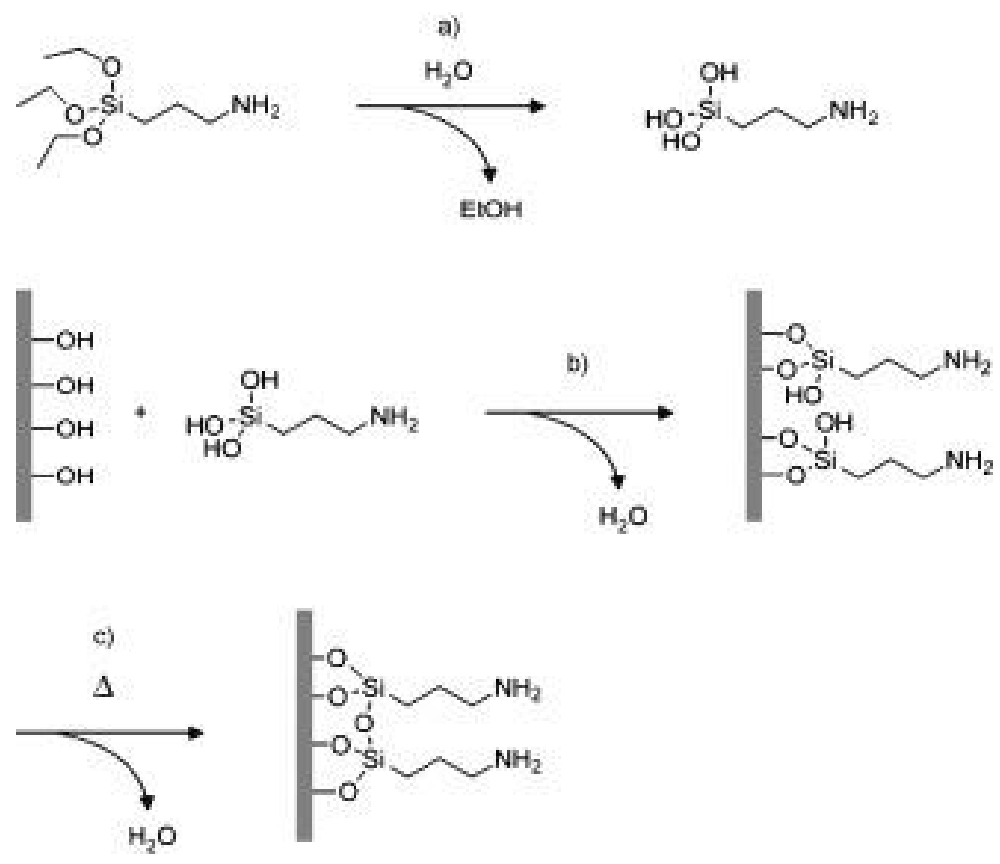

Figura 3.4. Mecanismo propuesto para la reacción de silanización en superficies planas. Adaptado de [13].

Para preparar Si nps silanizadas, se colocó en un balón de 3 bocas que contenía las cantidades adecuadas de Si nps en suspensión en tolueno y del precursor orgánico según se indicó en 3.3 completando con tolueno hasta llegar a volumen $100.00 \mathrm{ml}$. Se agregaron $500 \mu \mathrm{l}$ de agua sobre la mezcla y luego se calentó lentamente hasta llegar a reflujo $(\sim 110 \mathrm{C})$. La reacción se llevó a cabo durante ca. $20 \mathrm{hs}$, para luego retirar el calentamiento y enfriar lentamente. Finalmente, las $\mathrm{Si}$ nps fueron aisladas y purificadas por diálisis y ciclos de evaporación/disolución en el solvente de trabajo. 


\subsection{Síntesis de Au nps}

Existen diversos métodos para la síntesis de Au nps, sin embargo los más populares son los desarrollados por Brust y Schiffrin [16] y Turkevich y Frens [17][18]. Estos métodos son muy efectivos al momento de producir Au nps con muy baja dispersión de tamaños en el rango de $1-10$ y $10-20 \mathrm{~nm}$. Sin embargo, para obtener Au nps de mayores tamaños se han utilizado otras estrategias. Una de las más interesantes consiste en el crecimiento de semillas previamente sintetizadas, usando reductores que si bien son termodinámicamente capaces de reducir $\mathrm{Au}^{3+}$ la cinética de la reacción es lenta, salvo la presencia de superficies que catalicen el proceso. Dentro de este tipo de alternativas está la reducción de $\mathrm{HAuCl}_{4}$ con hidroxilamina $\left(\mathrm{NH}_{2} \mathrm{OH}\right)$ en presencia de semillas de Au [19].

\subsubsection{Síntesis de Au nps de (22 \pm 3 ) por el método de Turkecivh - Frens (Au 32)}

En primer lugar, se sintetizaron Au nps de $(22 \pm 3)$ nm usando una modificación del método de Turkevich-Frens, reportada por Haiss et al. [20]. Para esto, en un balón de reacción se colocaron $65.00 \mathrm{ml}$ de una solución acuosa recién preparada de $\mathrm{HAuCl}_{4} 254 \mu \mathrm{M}$. Esta solución se calentó a ebullición con agitación vigorosa y luego se agregaron $6.50 \mathrm{ml}$ de citrato de sodio $40 \mathrm{mM}$. La solución paso de ser amarilla a roja, color propio de las Au nps. La mezcla se mantuvo en ebullición por 10 minutos y luego se dejó enfriar a temperatura ambiente, manteniendo la agitación por 30 minutos más. Estas Au nps fueron dializadas contra solvente puro y luego fueron usadas como semillas en los pasos siguientes de crecimiento.

\subsubsection{Síntesis de Au nps de (68 \pm 4 ) por el método de crecimiento de semillas (Au 56)}

En la etapa de crecimiento, se empleó $\mathrm{NH}_{2} \mathrm{OH}$ como reductor. En principio, se agregaron volúmenes conocidos de soluciones acuosas de semillas de $\mathrm{Au}$ y $\mathrm{NH}_{2} \mathrm{OH}$ fresca en un matraz erlenmayer, y la mezcla se agitó vigorosamente a temperatura ambiente. Sobre esta se agregó $\mathrm{HAuCl}_{4}$, con lo cual se dio inicio al crecimiento, el cual puede verificarse a ojo desnudo por el cambio en la tonalidad de la dispersión de Au nps o midiendo el espectro de extinción de una muestra.

En un paso típico de crecimiento, se colocaron $7.50 \mathrm{ml}$ de semillas Au nps de concentración 3 nM junto a 4.00 $\mathrm{ml}$ de $\mathrm{NH}_{2} \mathrm{OH} 40 \mathrm{mM}$ y se completó con agua ultrapura hasta $75.00 \mathrm{ml}$. Esta mezcla se agitó a temperatura ambiente, y luego se agregaron $100 \mu \mathrm{l}$ de $\mathrm{HAuCl}_{4} 254 \mu \mathrm{M}$. Se observó un cambio inmediato en la tonalidad de la dispersión de Au nps. La reacción se dejó en agitación por 30 minutos, y posteriormente se dializó contra solvente puro para remover el exceso de $\mathrm{NH}_{2} \mathrm{OH}$. Las Au nps están cubiertas por citrato, que permite su estabilidad coloidal y evita su coalescencia. Las muestras fueron almacenadas a $4 \mathrm{C}$ hasta su uso.

\subsubsection{Síntesis de Au nps de (110 \pm 13$)$ por el método de crecimiento de semillas (Au 87)}

Se procedió de forma similar a 3.4.2., cambiando las cantidades empleadas de los precursores. Se emplearon $7.50 \mathrm{ml}$ de semillas Au nps de concentración $3 \mathrm{nM}$ junto a $4.00 \mathrm{ml}$ de $\mathrm{NH}_{2} \mathrm{OH} 40 \mathrm{mM}$ (volumen final $75.00 \mathrm{ml}$ ) y posteriormente se agregaron $300 \mu \mathrm{l}$ de $\mathrm{HAuCl} 4254 \mu \mathrm{M}$. Las Au nps obtenidas se aislaron y purificaron como se comentó en 3.4.2.

\subsubsection{Determinación de la concentración de Au nps}

Para estimar la concentración analítica de Au nps se utilizó el método reportado por Haiss y colaboradores [21]. Se registró el espectro de atenuación UV-vis para dispersiones madre de Au nps de diámetro d manteniendo las condiciones de adquisición constantes. Bajo esta condición, usando los valores de A para el máximo de la banda de resonancia del plasmón superficial, $A_{\text {spr, }}$ y para $\lambda=450 \mathrm{~nm}, A_{450}$, puede estimarse tanto $d$ (en $\mathrm{nm}$ ) como la concentración numérica $\mathrm{N}$ (en $\mathrm{M}$ ): 


$$
\begin{aligned}
& \mathrm{d}=\exp \left(B_{1} \frac{A_{\text {spr }}}{A_{450}}-B_{2}\right) \\
& \mathrm{N}=\frac{A_{450} * 10^{14}}{d^{2}\left[-0.295+1.36 * \exp \left(-\left(\frac{d-96.8}{78.2}\right)^{2}\right)\right]}
\end{aligned}
$$

Usando este método, el error relativo en la estimación de d es de hasta el 10\% y del 15\% para N, comparando con las concentraciones determinadas por ICP - AES.

\subsection{Unión de colorantes empleando enlaces amida}

La formación de enlaces amida es fundamental en la naturaleza, debido a su presencia masiva en péptidos y proteínas. El enlace amida presenta múltiples vías para su obtención, siendo clave al momento de elegir los subproductos formados y la facilidad para el aislamiento, posibilidad de formación de mezclas racémicas 0 selectividad isomérica o la compatibilidad con grupos protectores. Esta variedad de acercamientos está muy bien documentada en la literatura [22], y permite la elección del enfoque sintético más útil según el sistema a preparar, disponibilidad de reactivos, etc.

En general, la formación de enlaces amida procede a través de dos pasos sintéticos: Un paso de activación donde se forma un compuesto de acilo reactivo, y posteriormente una aminólisis donde el acilo reactivo es atacado por la amina a unir. Existen diversos reactivos los cuales forman compuestos acilos, en esta tesis se usaron en particular diciclohexilcarbodiimida (DCC) junto a N-hidroxisuccinimida (NHS), o cloruro de oxalilo. Para cada caso se empleó una estrategia particular, considerando las distintas condiciones de reacción y los subproductos formados.

En todos los casos se emplearon Si nps cubiertas con propilamina en su superficie, las que se mezclaron con el compuesto acilo reactivo.

En el caso de amidas formadas usando DCC/NHS, se mezcló en $10.00 \mathrm{ml}$ de DMF seco el precursor ácido con DCC y NHS en exceso ( $n_{\text {соон: }} n_{D C c}$ de 1:1.5) y se dejó en agitación suave por 12 hs en oscuridad, en atmósfera inerte de Ar y a temperatura ambiente. Luego de esto, se agregaron las Si nps aminadas en suspensión de DMF, en cantidad necesaria para garantizar $n_{\text {cooH: }} n_{\text {si nps }}$ cercano a 1:1, y se mantuvo la agitación en las mismas condiciones por 12 hs más. Finalmente, el subproducto formado (dicidlohexilurea, DCU) puede removerse por filtración, ya que forma un precipitado insoluble. La suspensión resultante fue dializada contra solvente puro y almacenada a $4 \mathrm{C}$ y en oscuridad hasta su uso.

Las reacciones usando cloruro de oxalilo se llevaron a cabo en condiciones similares a las de DCC/NHS, con diferencias al momento de la purificación. El precursor ácido y el cloruro de oxalilo (exceso de 1:1.5) fueron disueltos en $10.00 \mathrm{ml}$ de DMF seco y mezclados por agitación durante 12 hs en oscuridad, en atmósfera inerte de Ar y a temperatura ambiente. Luego se colocaron las Si nps aminadas en suspensión de DMF en cantidad necesaria para garantizar $n_{\text {соOH: }} n_{\text {Si nps }}$ cercano a 1:1. y se mantuvo la agitación en las mismas condiciones por 12 hs más. Como los subproductos en este caso son gases, directamente se dializó la suspensión resultante contra solvente puro y se almacenó a $4 \mathrm{C}$ en oscuridad hasta su uso.

\subsection{Referencias}

[1] M. J. Llansola Portolés, F. R. Nieto, D. B. Soria, J. I. Amalvy, P. J. Peruzzo, D. O. Mártire, M. Kotler, O. Holub, and M. C. Gonzalez, "Photophysical properties of blue-emitting silicon nanoparticles," J. 
Phys. Chem. C, vol. 113, pp. 13694-13702, 2009.

[2] M. J. Sailor, Porous Silicon in Practice: Preparation, Characterization and Applications. 2012.

[3] G. Bomchil, A. Halimaoui, and R. Herino, "Porous silicon: The material and its applications in siliconon-insulator technologies," Appl. Surf. Sci., vol. 41-42, no. C, pp. 604-613, 1989.

[4] X. G. Zhang, "Morphology and Formation Mechanisms of Porous Silicon," J. Electrochem. Soc., vol. 151, no. 1, p. C69, 2004.

[5] Z. F. Li, M. T. Swihart, and E. Ruckenstein, "Luminescent silicon nanoparticles capped by conductive polyaniline through the self-assembly method," Langmuir, vol. 20, no. 19, pp. 1963-1971, 2004.

[6] M. Rosso-Vasic, E. Spruijt, and B. Van Lagen, Alkyl-Functionalized Oxide-Free Silicon Nanoparticles: Synthesis and Optical Properties Cited by me. 2008.

[7] D. Neiner, H. W. Chiu, and S. M. Kauzlarich, "Low-temperature solution route to macroscopic amounts of hydrogen terminated silicon nanoparticles," J. Am. Chem. Soc., vol. 128, pp. 11016-11017, 2006.

[8] R. K. Baldwin, K. a. Pettigrew, J. C. Garno, P. P. Power, G. Y. Liu, and S. M. Kauzlarich, "Room temperature solution synthesis of alkyl-capped tetrahedral shaped silicon nanocrystals," J. Am. Chem. Soc., vol. 124, pp. 1150-1151, 2002.

[9] K. Pettigrew and Q. Liu, "Solution synthesis of alkyl-and alkyl/alkoxy-capped silicon nanoparticles via oxidation of Mg2Si," Chem. ..., no. 31, pp. 4005-4011, 2003.

[10] F. Hua, M. T. Swihart, and E. Ruckenstein, "Efficient surface grafting of luminescent silicon quantum dots by photoinitiated hydrosilylation," Langmuir, vol. 21, no. 18, pp. 6054-6062, 2005.

[11] E. V. Rogozhina, D. a. Eckhoff, E. Gratton, and P. V. Braun, "Carboxyl functionalization of ultrasmall luminescent silicon nanoparticles through thermal hydrosilylation," J. Mater. Chem., vol. 16, p. 1421, 2006.

[12] J. H. Warner, H. Rubinsztein-Dunlop, and R. D. Tilley, "Surface morphology dependent photoluminescence from colloidal silicon nanocrystals," J. Phys. Chem. B, vol. 109, pp. 19064-19067, 2005.

[13] J. M. Buriak, "Illuminating silicon surface hydrosilylation: An unexpected plurality of mechanisms," Chem. Mater., vol. 26, no. 1, pp. 763-772, 2014.

[14] P. Jonkheijm, D. Weinrich, H. Schröder, C. M. Niemeyer, and H. Waldmann, "Chemical strategies for generating protein biochips," Angew. Chemie - Int. Ed., vol. 47, no. 50, pp. 9618-9647, 2008.

[15] J. Zou, R. K. Baldwin, K. a. Pettigrew, and S. M. Kauzlarich, "Solution synthesis of ultrastable luminescent siloxane-coated silicon nanoparticles," Nano Lett., vol. 4, pp. 1181-1186, 2004.

[16] M. Brust, D. Bethell, D. J. Schiffrin, and R. Whyman, "Synthesis of Thiol-derivatised Gold Nanoparticles in," pp. 801-802, 2000.

[17] J. Turkevich, P. C. Stevenson, and J. Hillier, "A study of the nucleation and growth processes in the synthesis of colloidal gold," Discuss. Faraday Soc., vol. 11, no. c, pp. 55-75, 1951.

[18] G. Frens, "Controlled Nucleation for the Regulation of the Particle Size in Monodisperse Gold Suspensions," Nat. Phys. Sci., vol. 241, no. 105, pp. 20-22, 1973. 
[19] K. R. Brown and M. J. Natan, "Hydroxylamine seeding of colloidal Au nanoparticles in solution and on surfaces," Langmuir, vol. 14, no. 4, pp. 726-728, 1998.

[20] W. Haiss, N. T. K. Thanh, J. Aveyard, and D. G. Fernig, "Determination of Size and Concentration of Gold Nanoparticles from UV - Vis Spectra Determination of Size and Concentration of Gold Nanoparticles from UV - Vis Spectra," no. October, pp. 1-7, 2015.

[21] W. Haiss, N. T. K. Thanh, J. Aveyard, and D. G. Fernig, "Determination of Size and Concentration of Gold Nanoparticles from UV - Vis Spectra Determination of Size and Concentration of Gold Nanoparticles from UV - Vis Spectra," vol. 79, no. October, pp. 4215-4221, 2015.

[22] C. A. G. N. Montalbetti and V. Falque, "Amide bond formation and peptide coupling," Tetrahedron, vol. 61, no. 46. pp. 10827-10852, 2005. 


\section{Parte II}

Nanopartículas y Ensamblados

Supramoleculares 
- 78 - 


\section{Capítulo 4: Fotofísica de Si nps: El efecto de la superficie}

En este capítulo se presentan los estudios realizados a fin de comprender el efecto de la morfología, la modificación superficial y la temperatura sobre la fotoluminiscencia ( $P L$, del inglés photoluminescence) de Si nps de tamaños entre 1 y $2 \mathrm{~nm}$; sintetizadas empleando distintas estrategias. A tal fin, las Si nps fueron sintetizadas usando una vía electroquímica (de "arriba hacia abajo") y procesos de química húmeda (de "abajo hacia arriba"). Las nps obtenidas fueron derivatizadas usando reacciones de sililación o silanización, logrando

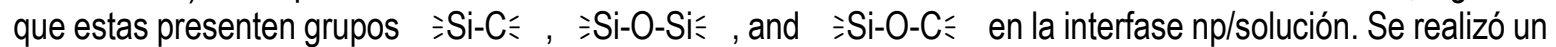
análisis detallado de las matrices de emisión-excitación (EEM, del inglés excitation-emission matrices), el cual indicó claramente que distintos arreglos atómicos en la superficie contribuyen a la diferencia de energía entre la banda de conducción y de valencia (BG, del inglés Band Gap). Particularmente, las partículas con grupos

汭-O-Si i en la interfase mostraron PL independiente de la estructura cristalina o de la derivatización superficial con distintas moléculas orgánicas. Se probó que los tiempos de vida y la distribución del espectro de $\mathrm{PL}$ de todas las Si nps sintetizadas eran invariables frente a cambios de temperatura en el rango de $270-330$ $\mathrm{K}$, además de mostrar una reducción significativa de la $\mathrm{PL}$ al incrementar la temperatura; este comportamiento puede explicarse con la existencia de un equilibrio térmico entre conformaciones emisivas y oscuras de las partículas. Los resultados observados son importantes al momento de optimizar el uso de Si nps como sensores ópticos y agentes terapéuticos en sistemas biológicos.

\subsection{Introducción}

Las Si nps de 1 - $5 \mathrm{~nm}$ han recibido mucha atención ya que combinan emisión fotoluminiscente en la región visible y en el IR cercano del espectro electromagnético, con la capacidad de generar oxígeno singlete $\left({ }^{1} \mathrm{O}_{2}\right)$ y anión radical superóxido $\left(\mathrm{O}_{2}^{-*}\right)[1][2]$. A su vez, el Silicio nanoestructurado presenta propiedades interesantes como biocompatibilidad, biodegradabilidad y una amplia química superficial apta para la administración de fármacos [3][4]. La funcionalización de Si nps con grupos funcionales activos, biomoléculas o polímeros biocompatibles es un paso clave en el desarrollo de muchas aplicaciones tecnológicas. La selección adecuada de grupos superficiales confiere a las partículas propiedades útiles como dispersabilidad en medios específicos, protección contra la oxidación, direccionalidad específica y prevención frente a la opsonización [5].

El trabajo con Si nps demostró que la cristalinidad y la estructura y química superficial juegan un rol importante en su comportamiento fotofísico. Interpretar y clasificar la gran cantidad de BG y corrimientos de Stokes (SS, del inglés Stokes shift) publicados es difícil debido a las grandes divergencias en la síntesis de muestras monodispersas con propiedades superficiales bien definidas, ya que una variedad de grupos (por ejemplo -Si$\mathrm{OH},>\mathrm{Si}=\mathrm{O}, \quad=\mathrm{Si}-\mathrm{O}-\mathrm{Si}=\quad, \quad=\mathrm{Si}-\mathrm{H}, \quad=\mathrm{Si}-\mathrm{C} \leqslant$ ) pueden estar presentes, conformando una interfase compleja en el producto diseñado. A su vez, la fotofísica fundamental de las Si nps aún no es del todo comprendida como en el caso de semiconductores con transiciones directas como CdSe [6].

Cálculos basados en la teoría del funcional de la densidad asumiendo una barrera finita para el potencial de confinamiento aplicados a clusters de Si saturados con hidrógeno predicen una variación suave de BG con el tamaño del cluster [7]. Sin embargo, experimentalmente se observaron BG mayores para clusters cristalinos, lo cual se explicó como una consecuencia de la simetría y la disminución en la distorsión angular en enlaces $\mathrm{Si}-\mathrm{Si}$ [8]. Estos autores hallaron que la localización del HOMO y el LUMO se encontraba en el núcleo para superficies sin reconstruir (por ejemplo, $\mathrm{Si}_{35} \mathrm{H}_{36}$ con superficie similar a la estructura del seno del cristal), mientras que en superficies reconstruidas la localización se daba en la superficie (como en el caso de $\mathrm{Si}_{29} \mathrm{H}_{24}$ ). Además, el trabajo tanto computacional como experimental con Si nps, demostró la importancia fundamental de la química superficial al considerar el comportamiento fotofísico [6][9][10]. Particularmente en núcleos $\mathrm{Si}_{29}$ y 
$\mathrm{Si}_{47}$, los grupos $\mathrm{Si}-\mathrm{OH}$ y Si-O-Si muestran relativamente poca influencia en la posición de los bordes de las bandas 0 en $\mathrm{EG}$, mientras que estructuras $>\mathrm{Si}=\mathrm{O}$ los afectan significativamente. El aumento en el cubrimiento superficial causa también cambios en EG [9]. Estudios recientes sugieren que las estructuras Si-O-Si en superficies curvas de Si nps con diámetros menores a $3 \mathrm{~nm}$, pero no en el caso de Si nps de $3-4 \mathrm{~nm}$ de diámetro, conllevan a la localización de niveles en medio del EG. Como consecuencia, se observa un corrimiento al rojo en el espectro de PL de Si nps oxidadas de pequeños tamaños [11].

En la bibliografía se sugiere que la dependencia con la temperatura de la PL de Si nps depende fuertemente del tamaño [12][13][14]. Se reportó que Si nps sintetizadas usando diversas rutas mostraron un aumento en la intensidad de PL al disminuir la temperatura hasta ca. 60 - $80 \mathrm{~K}$. Mayores descensos en la temperatura disminuyen rápidamente la intensidad de PL y un aumento en el tiempo de vida de PL. Estas observaciones siempre fueron discutidas en términos de la competencia entre las constantes (dependientes de la temperatura) de decaimiento radiativo $\left(\mathrm{k}_{\mathrm{r}}\right)$ y no radiativo $\left(\sum_{i} \mathrm{k}_{\mathrm{nr}, \mathrm{i}}\right)$ para estados excitados altamente localizados, como en el caso de defectos en nanocristales o en la interfase $\mathrm{Si}_{/} \mathrm{SiO}_{2}$.

A continuación, se presentan los resultados sobre el efecto de la derivatización superficial y la temperatura en la PL de Si nps sintetizadas usando diversas vías, en un intento de entender el efecto de la superficie en la PL de las partículas. La comprensión de estos efectos es necesaria para optimizar el uso de Si nps como sensores ópticos y agentes terapéuticos en sistemas biológicos. A tal fin se sintetizaron Si nps por vías electroquímicas (de "arriba hacia abajo") y de química húmeda (de "abajo hacia arriba"), y posteriormente fueron derivatizadas usando reacciones de sililación y silanización, para obtener finalmente Si nps con grupos -NH2 terminales y diferentes estructuras químicas en la interfase ( $=\mathrm{Si}-\mathrm{O}-\mathrm{Si} \leqslant$, $\quad$ Si-C o $\quad$ Si-O-C $\leqslant$ ).

\subsection{Aspectos experimentales y metodológicos}

Los detalles referidos a síntesis y métodos experimentales han sido desarrollados en mayor extensión en los capítulos 2 y 3 . A continuación, se comentarán de forma breve los procedimientos relacionados al presente capítulo.

Las Si nps estudiadas en este capítulo fueron sintetizadas tanto por vías de "arriba hacia abajo" como de "abajo hacia arriba". Las vías TD fueron una adaptación del grabado electroquímico con HF de pSi [1]. Brevemente, obleas de Si de $20 \times 20 \mathrm{~mm}^{2}(<100>$ tipo $\mathrm{p}$, con resistividad entre 1.0 y $10.0 \Omega \mathrm{cm}$ ) limpias según se mencionó en el capítulo 3, fueron anodizadas ( $\mathrm{i} \sim 20 \mathrm{~mA}$ ) en una celda de teflon conteniendo la solución electrolítica ( $16 \%$ $\mathrm{HF}, 16 \% \mathrm{MeOH}$ en agua ultrapura), usando un electrodo de anillo de platino como contraelectrodo. Luego de la anodización, el blanco de Si se enjuagó con $\mathrm{MeOH}$ y tolueno, y posteriormente se almacenó en un baño de tolueno en atmósfera inerte. Luego, este sistema fue colocado en un baño de ultrasonidos por $2 \mathrm{hs}$. La suspensión resultante se separó por filtración usando membranas de $220 \mathrm{~nm}$ de PVDF (MILLEX-HV, Millipore), conservándose tanto el sólido como el filtrado para obtener distintas Si nps.

El residuo sólido se suspendió en una solución 3\% v/v de HF en $\mathrm{MeOH}$, se sonicó y almacenó en la oscuridad por 20 hs para romper los aglomerados de pSi [18]. Luego se agregó agua, y esta suspensión fue puesta en contacto con ciclohexano para extraer las Si nps hidrofóbicas, terminadas en hidrógeno en su superficie. El extracto orgánico se calentó por 9 hs, y finalmente se evaporó el solvente para redisolver las Si nps en tolueno. Estas nps serán denominadas TD Si nps. En caso de ser requerido, las TD Si nps fueron oxidadas suavemente en dispersiones en contacto con aire durante varias semanas, lo cual llevó a la formación de partículas con superficies oxidadas, denominadas SiOx-TD Si nps. A su vez, del filtrado obtenido luego del grabado electroquímico se obtuvieron Si nps con una capa superficial de óxidos de Si y terminaciones Si-H. Estas np se llamaron SiOx-TD' Si nps.

La estrategia BU fue una adaptación de la reducción de SiCl4 con LiAlH4 en presencia de micelas invertidas de $\mathrm{TOABr}$ [16]. Estas partículas luego de sintetizadas presentaron superficies terminadas con $\mathrm{H}$ - y $\mathrm{Cl}$ - y fueron llamadas BU Si nps. A su vez, los grupos superficiales fueron oxidados a Si-OH al almacenar las muestras en 
atmósfera equilibrada con aire por dos semanas, y confirmado por la aparición de una banda cerca de 3400 $\mathrm{cm}-1$ en el espectro FTIR, asignable a estiramientos $\mathrm{Si}-\mathrm{OH}$. Estas nps fueron denominadas $\mathrm{SiOx}-\mathrm{BU}$ Si nps.

Las Si nps sintetizadas fueron cubiertas con moléculas orgánicas usando reacciones de sililación foto-iniciadas o por silanización por vía térmica. Las reacciones de sililación se llevaron adelante en reactores cilíndricos de cuarzo, iluminados con radiación de $254 \mathrm{~nm}$ de 8 lámpraras por $5 \mathrm{hs}$, o usando radiación de una lámpara de $350 \mathrm{~nm}$ durante toda una noche. Las mezclas de reacción fueron preparadas usando los compuestos insaturados de interés en concentraciones de $2 \%$ v/v en tolueno, sobre los cuales se agregaron las Si nps a derivatizar suspendidas en tolueno. En particular, se empleó 2-propenilamina (AA) para derivatizar tanto TD Si nps como BU Si nps, obteniéndose superficies terminadas con grupos propilamina. Estas nuevas np fueron llamadas PA-TD Si nps y PA-BU Si nps, respectivamente. A su vez, se empleó 2-metil-2-propenoato de metilo (MA) para cubrir SiOx-TD' Si nps, obteniendo MASiOx-TD' Si nps.

El procedimiento empleado para la silanización consistió en acoplar APTES a grupos Si-OH terminales [18][19]. Para esto, suspensiones de nps oxidadas en su superficie (SiOx-BU Si nps y SiOx-TD Si nps) en tolueno con $1 \% \mathrm{v} / \mathrm{v}$ de APTES fueron agitadas a temperatura ambiente por $24 \mathrm{hs}$. Los reactivos en exceso se removieron por diálisis. Las Si nps modificadas con APTES fueron llamadas PASiOx-BU Si nps y PASiOx-TD Si nps, respectivamente.

Para ser más claros, las vías sintéticas empleadas se resumen en la figura 4.1. 


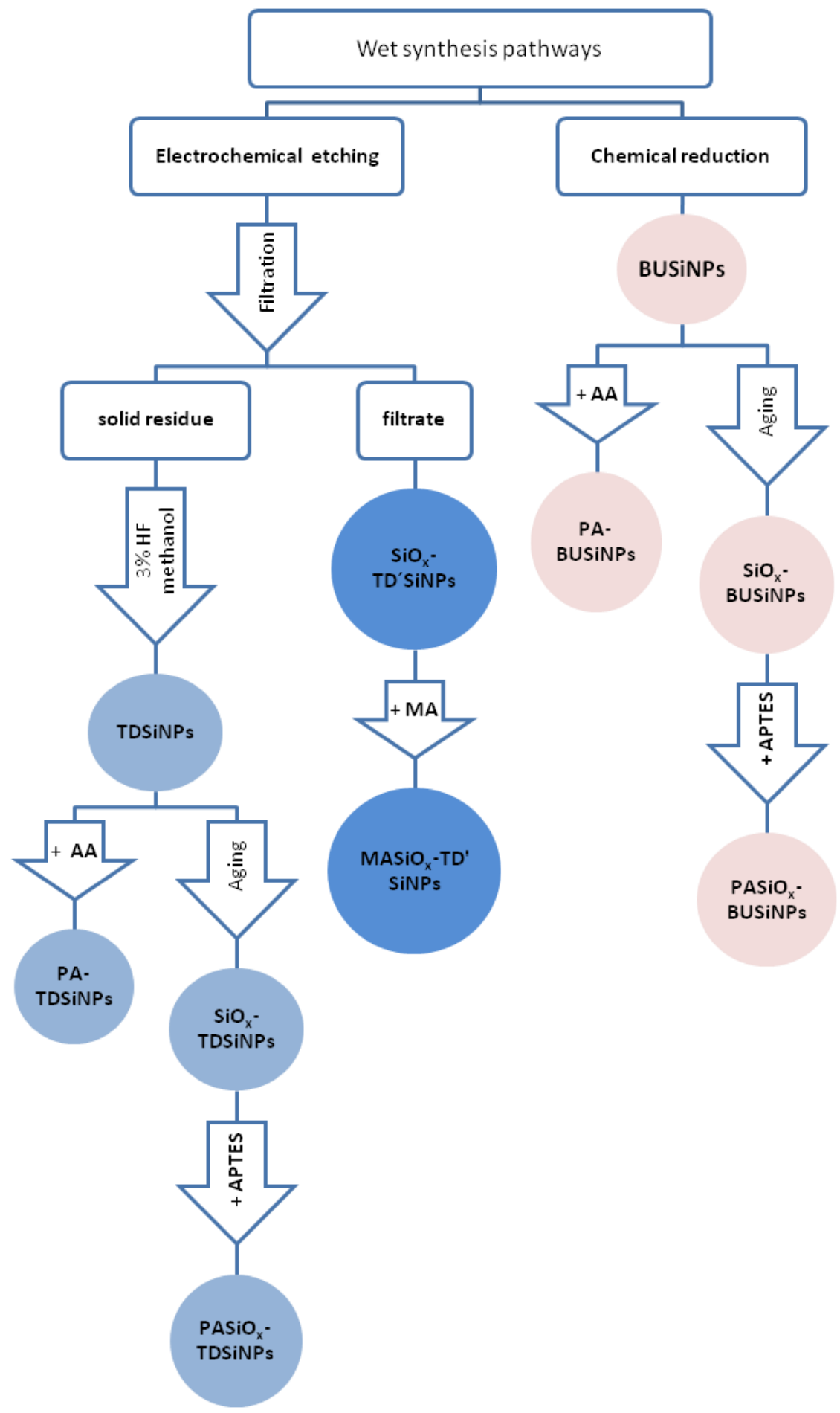

Figura 4.1. Rutas sintéticas para la obtención de las Si nps empleadas en los experimentos discutidos en este capítulo. 


\subsection{Resultados y discusión}

\subsubsection{Síntesis y caracterización}

Se sintetizaron Si nps usando estrategias tanto TD como BU, obteniendo nanopartículas cristalinas con superficies terminadas en hidrógeno (TD Si nps), o amorfas y terminadas con $\mathrm{H}$ - y $\mathrm{Cl}$ - (BU Si nps). Estas partículas fueron tratadas a fin de cubrir su superficie con grupos terminales propilamina, obteniendo PA-TD Si nps y PA-BU Si nps, respectivamente, o se dejaron añejar en suspensiones equilibradas con aire para formar nanopartículas oxidadas superficialmente, las cuales se llamó SiOx-TD Si nps y SiOx-BU Si nps. Sobre estas últimas se llevaron a cabo reacciones de silanización con APTES, obteniéndose Si nps terminadas con grupos propilamina, pero con interfases oxidadas de forma controlada. Estas nuevas nanopartículas se denominaron PASiOx-TD Si nps y PASiOx-BU Si nps. A su vez, las partículas SiOx-TD' Si nps fueron modificadas con 2metil-2-propenoato de metilo para obtener MASiOx-TD' Si nps. Las reacciones empleadas para modificación superficial, en el caso particular de las TD Si nps y SiOx-TD' Si nps se resume en la figura 4.2, en la cual también se esquematiza su composición superficial.

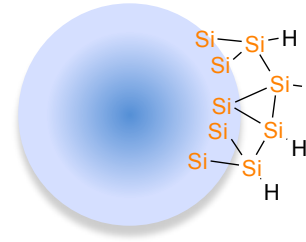

TDSiNP

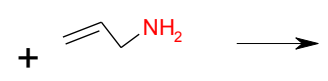

2-propen 1-amine

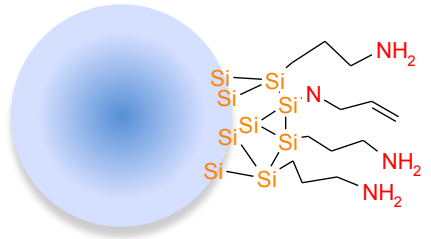

PA-TDSiNP

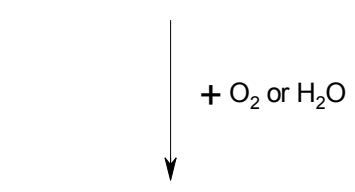

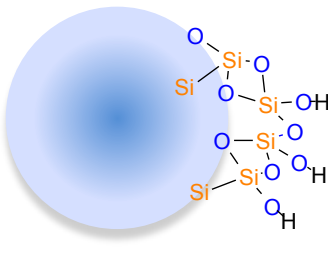

$\mathrm{SiO}_{\mathrm{x}}-\mathrm{TDSiNP}$

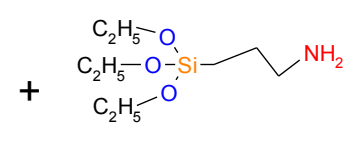

(3- aminopropyl)triethoxysilane

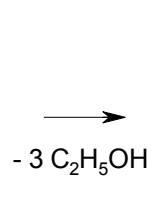

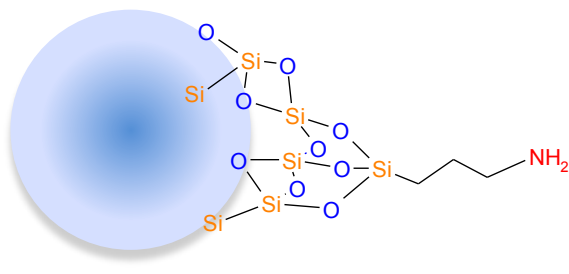

$\mathrm{PASiO}_{\mathrm{x}}-\mathrm{SiNP}$

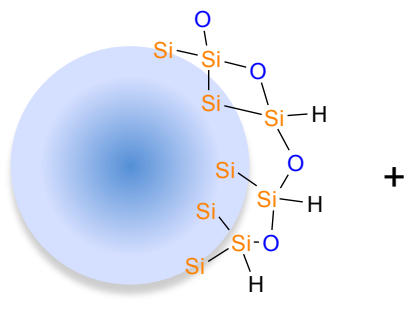

$\mathrm{SiO}_{\mathrm{x}}-\mathrm{TD}$ 'SiNP

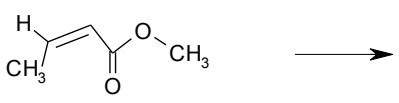

methyl 2-methylprop-2-enoate

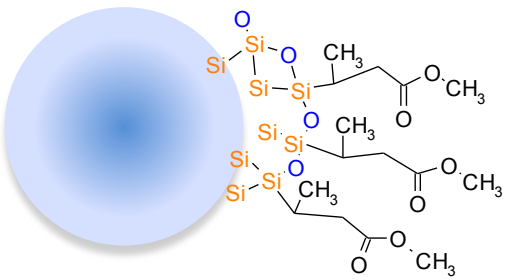

$\mathrm{MASiO}_{\mathrm{x}}-\mathrm{TD}^{\prime} \mathrm{SiNP}$

Figura 4.2. Reacciones empleadas en modificaciones superficiales post-síntesis, en el caso de TD Si nps y SiOx-TD' Si nps. 
Mediante el análisis de micrografías TEM se determinó un tamaño promedio de $1.8 \mathrm{~nm}$ con una gran dispersión hacia tamaños mayores para las TD Si nps, como se ve en las figuras 4.3 (A) y 4.3 (B). De todos modos, un análisis detallado de las micrografías denota una importante aglomeración. De hecho, experimentos de microscopía de fuerza atómica usando TD Si nps terminadas con grupos mercaptopropilo sobre soportes de mica, y de microscopía de barrido por efecto túnel con estas mismas nanopartículas pero en soportes de Au (111) muestran distribuciones de $(1.7 \pm 0.3)$ y $(1.6 \pm 0.5) \mathrm{nm}$, respectivamente [20]. La figura 4.3 (C) permite observar la separación entre planos cristalinos de estas Si nps alrededor de $3.1 \pm 0.1 \AA$, en acuerdo con la distancia para planos (111) de Si [21][22]. En el caso de las BU Si nps se determinó un tamaño promedio de $(1.7 \pm 0.8)$ nm y no pudo encontrarse evidencia de cristalinidad, con lo cual se las asumió amorfas.
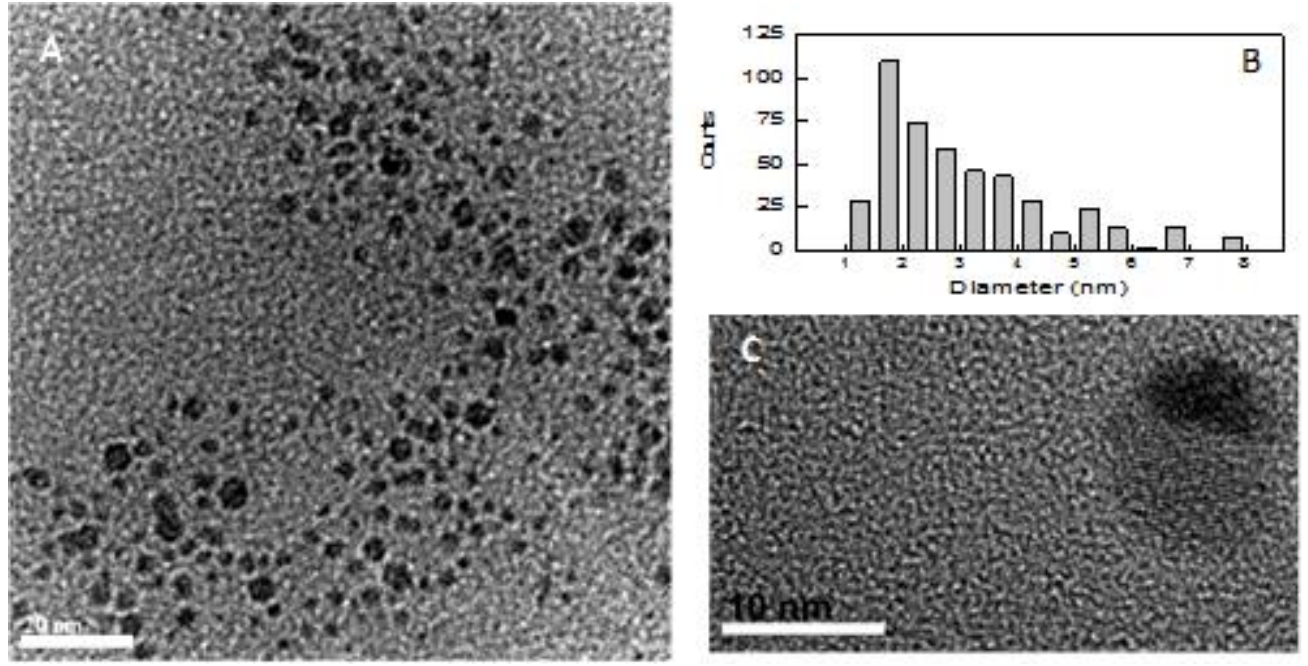

Figura 4.3. (A) Micrografía TEM de TD Si nps, (B) Histograma de tamaños asociado, a patir de 500 partículas sin contacto (C) Micrografía HR-TEM de partículas aglomeradas, en las cuales pueden distinguirse planos con orientación (111).

El análisis de los espectros XPS y FTIR de diferentes sistemas permitió la determinación de las composiciones superficiales promedio. La parte superior de la figura 4.4 (A) muestra el espectro FTIR de las PA-TD Si nps. Las señales a 3400 (débil), 4650-1580 y $910-665 \mathrm{~cm}^{-1}$ son características de aminas primarias y secundarias. El estiramiento C-N de las aminas alifáticas se observa a $1260 \mathrm{~cm}^{-1}$. La presencia de bandas a 1435, 1270, 805 y $740 \mathrm{~cm}^{-1}$ debido a deformaciones simétricas y asimétricas, deformación, estiramiento y balanceo de metilenos en $\mathrm{Si}-\mathrm{CH}_{2}$, respectivamente, como también absorciones en la región de $2970-2880 \mathrm{~cm}^{-1}$ debido a estiramientos y flexiones de metileno, confirma la unión del grupo propilamino a las TD Si nps [23][24]. El espectro XPS de alta resolución en la región del Si 2p de este sistema (Figura 4.4 B) puede deconvolucionarse considerando picos con energías de enlace a 99.70 (10\%), 100.7 (57\%) y 101.8 eV (33\%), característicos de entornos Si-O, Si-C y Si-N respectivamente [25][26][27]. A su vez, la señal en la región de $\mathrm{N}$ 1s puede describirse como un pico mayoritario a $399.4 \mathrm{eV}$ característico de grupos $\mathrm{C}^{-\mathrm{NH}_{2}}$. No se asignaron contribuciones debidas a N en entornos de Si (situado a $398 \mathrm{eV}$ ) [27]. Sin embargo, al analizar el espectro FTIR la banda a 880 puede asignarse a vibraciones Si-N [28]. Es posible la existencia de subproductos que presenten enlaces Si-N, según se evidencia en el mecanismo radicalario propuesto, explicado en el capítulo 3. 

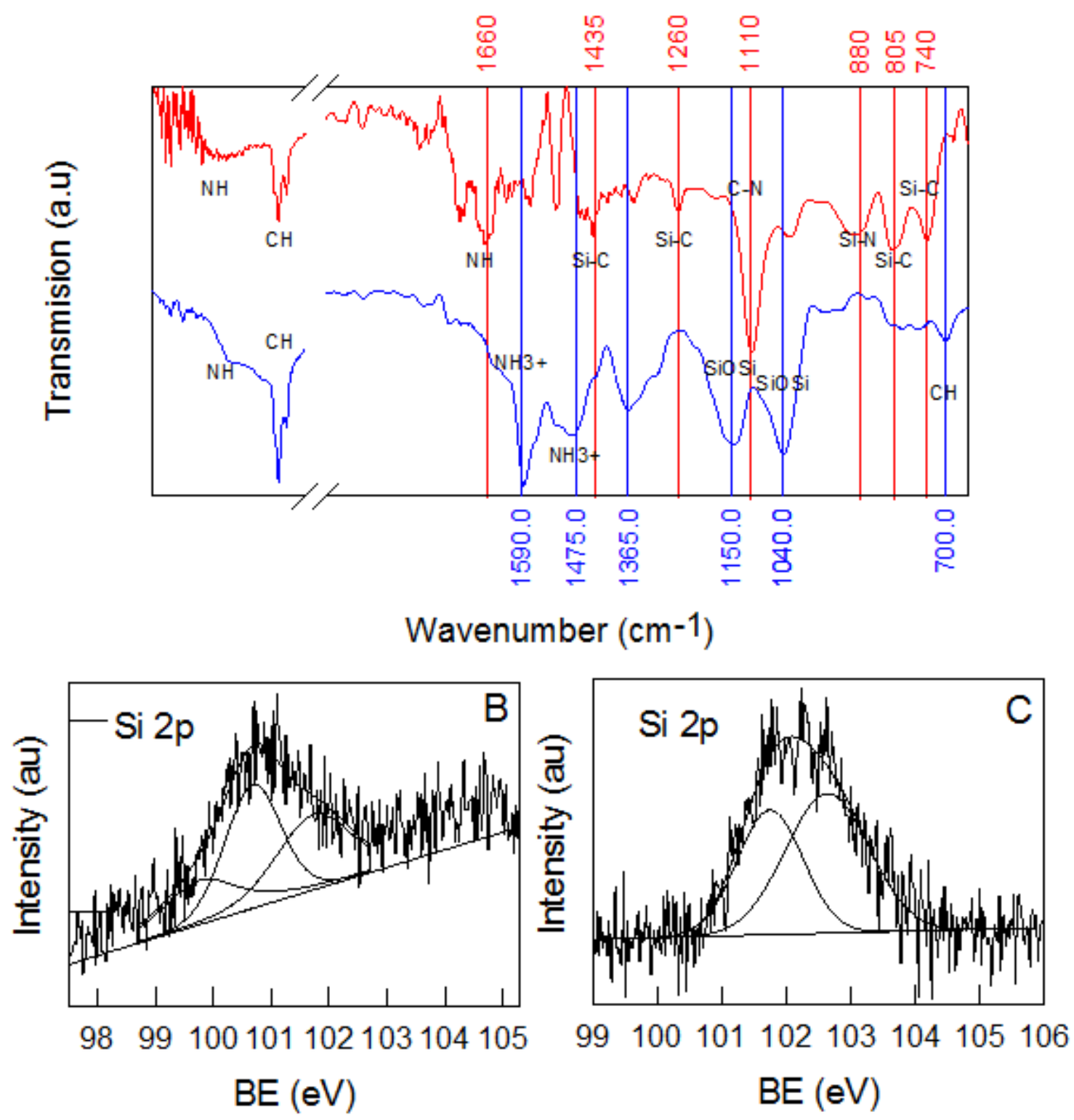

Figura 4.4. (A) Espectros FTIR de PA-TD Si nps (rojo) y PASiOx-TD Si nps (azul), depositadas desde suspensiones en toluene sobre soportes de Si. Las líneas verticales se colocaron para identificar fácilmente las posiciones de picos. (B) y (C) corresponden a los espectros XPS de alta resolución en la región de Si 2p para PA-TD Si nps y PASiOx-TD Si nps, respectivamente.

El espectro FTIR de PASiOx-TD Si nps (espectro inferior en la figura $4.4 \mathrm{~A}$ ) muestra bandas intensas en 1040 y $1150 \mathrm{~cm}^{-1}$ características de vibraciones $\mathrm{Si}-\mathrm{O}-\mathrm{Si}$ en distintos entornos, denotando partículas oxidadas. Las bandas en la región de $1450-1550 \mathrm{~cm}^{-1}$ pueden asignarse a vibraciones $\mathrm{N}-\mathrm{H}$ en aminas protonadas [29]. El pico a $1332 \mathrm{~cm}^{-1}$ se asignó a $\mathrm{CO}_{2}$ atrapado por grupos $\mathrm{NH}_{3}{ }^{+}$en superficie, en coincidencia a lo reportado para films de APTES [30]. A su vez, el espectro XPS de alta resolución en la región de Si $2 p$ muestra contribuciones con energías de enlace a 101.7 y $102.6 \mathrm{eV}$, que se asignaron a entornos $\mathrm{Si}(\mathrm{O}-)_{2}$ y $\mathrm{Si}(\mathrm{O}-)_{3}-\mathrm{C}$, de acuerdo con la estructura superficial propuesta para PASiOx-TD Si nps en la figura 4.2. La señal en la región de $\mathrm{N}$ 1s está centrada en $399.4 \mathrm{eV}$ y puede asociarse a entornos $\mathrm{C}-\mathrm{NH}_{2}$, y los picos en el FTIR debido a estiramientos y flexiones de metileno en las regiones $2880-2970 \mathrm{~cm}^{-1}$ y $1440-1465 \mathrm{~cm}^{-1}$, dan mayor sustento a la unión covalente de residuos orgánicos a la red de Si. La ausencia de señales alrededor de 104 eV en el espectro XPS 
de alta resolución de $\mathrm{Si} 2 \mathrm{p}$ y de bandas $\mathrm{IR}$ alrededor de $1250 \mathrm{~cm}^{-1}$ asociadas a $\mathrm{Si}=0$ en $\mathrm{SiO}_{2}$ masivo apoyan la hipótesis de formación de estructuras del tipo $\mathrm{SiO}_{2}$ en cantidad despreciable [33].

Las relaciones entre las señales de $\mathrm{Si}, \mathrm{O}, \mathrm{C}$ y $\mathrm{N}$ en el espectro XPS corregidas por los factores de sensibilidad instrumental permitieron obtener la composición promedio de las distintas Si nps preparadas, como se ve en la tabla 1, y de acuerdo con las estructuras propuestas en la figura 4.2. Al analizar los espectros FTIR y XPS para BU Si nps, PA-BU Si nps, SiOx-BU Si nps y PASiOx-BU Si nps se obtienen conclusiones similares. Debe mencionarse que para PA-TD Si nps se observa una pequeña absorción en el IR a $850 \mathrm{~cm}^{-1}$ y contribuciones a la señal Si 2p en XPS a 101.5 y 102.2 eV, característicos de Si(CxNy) y entornos Si-N [25][28]. En conclusión, la derivatización con grupos propilamina de Si nps pasivadas con $\mathrm{H}$ usando sililación foto-inciada a través de un mecanismo radicalario lleva a la formación de enlaces Si-N sin importar la estructura cristalina de la red de Si.

\begin{tabular}{|c|c|c|}
\hline Si np & Tamaño (nm) & Composición superficial media a \\
\hline TD Si nps & \multirow{4}{*}{$1.7 \pm 0.3$} & ----- \\
\hline PA-TD Si nps & & $\mathrm{Si}_{2} \mathrm{C}_{5} \mathrm{~N}_{4.4} \mathrm{H}_{\mathrm{y}}$ \\
\hline $\mathrm{SiO}_{x}-\mathrm{TD}$ Si nps & & ----- \\
\hline PASiOx-TD Si nps & & $\mathrm{Si}_{12} \mathrm{C}_{3.5} \mathrm{H}_{\mathrm{y}} \mathrm{N}_{1} \mathrm{O}_{\mathrm{x}}$ \\
\hline MASiOx-TD 'Si nps & $3 \pm 1 b$ & $\mathrm{Si}_{3} \mathrm{O}_{6}\left(\mathrm{C}_{5} \mathrm{O}_{2} \mathrm{H}_{\mathrm{y}}\right)_{1} \mathrm{~b}$ \\
\hline BU Si nps & \multirow{4}{*}{$1.7 \pm 0.8$} & ----- \\
\hline PA-BU Si nps & & ----- \\
\hline $\mathrm{SiO}_{x}-\mathrm{BU} \mathrm{Si}$ ns & & $\mathrm{Si}_{4.2} \mathrm{O}_{3.8} \mathrm{ClH}_{\mathrm{y}}$ \\
\hline PASiO $_{x}-\mathrm{BU}$ Si nps & & ---- \\
\hline
\end{tabular}

Tabla 4.1. Tamaño y composición superficial promedio de muestras obtenidas a través de distintas vías sintéticas y modificaciones post-síntesis. Las partículas con superficies oxidadas están marcadas en gris. a La fracción y de $\mathrm{H}$ no fue determinada. ${ }^{\mathrm{b}}$ Datos de [1].

\subsubsection{Experimentos fotofísicos}

La PL de distintas partículas en suspensiones de tolueno saturadas en Ar mostraron detalles particulares fuertemente dependientes de los grupos superficiales y el proceso sintético empleado. A su vez, la dependencia del espectro de PL con la longitud de onda de excitación para una suspensión determinada indica la contribución de partículas con distintos tamaños, morfología, química superficial o grado de oxidación dentro de la misma muestra. La contribución de distintas especies emisoras a la EEM experimental se analizó asumiendo la existencia de cromóforos simples en una única forma que muestran un espectro de PL invariante con la longitud de onda de excitación [16]. En la figura 4.5 se muestran los espectros de emisión y excitación de la PL de los emisores contribuyentes obtenidos en el análisis multilineal de las EEMs de suspensiones en tolueno saturadas en Ar para las distintas Si nps sintetizadas. 

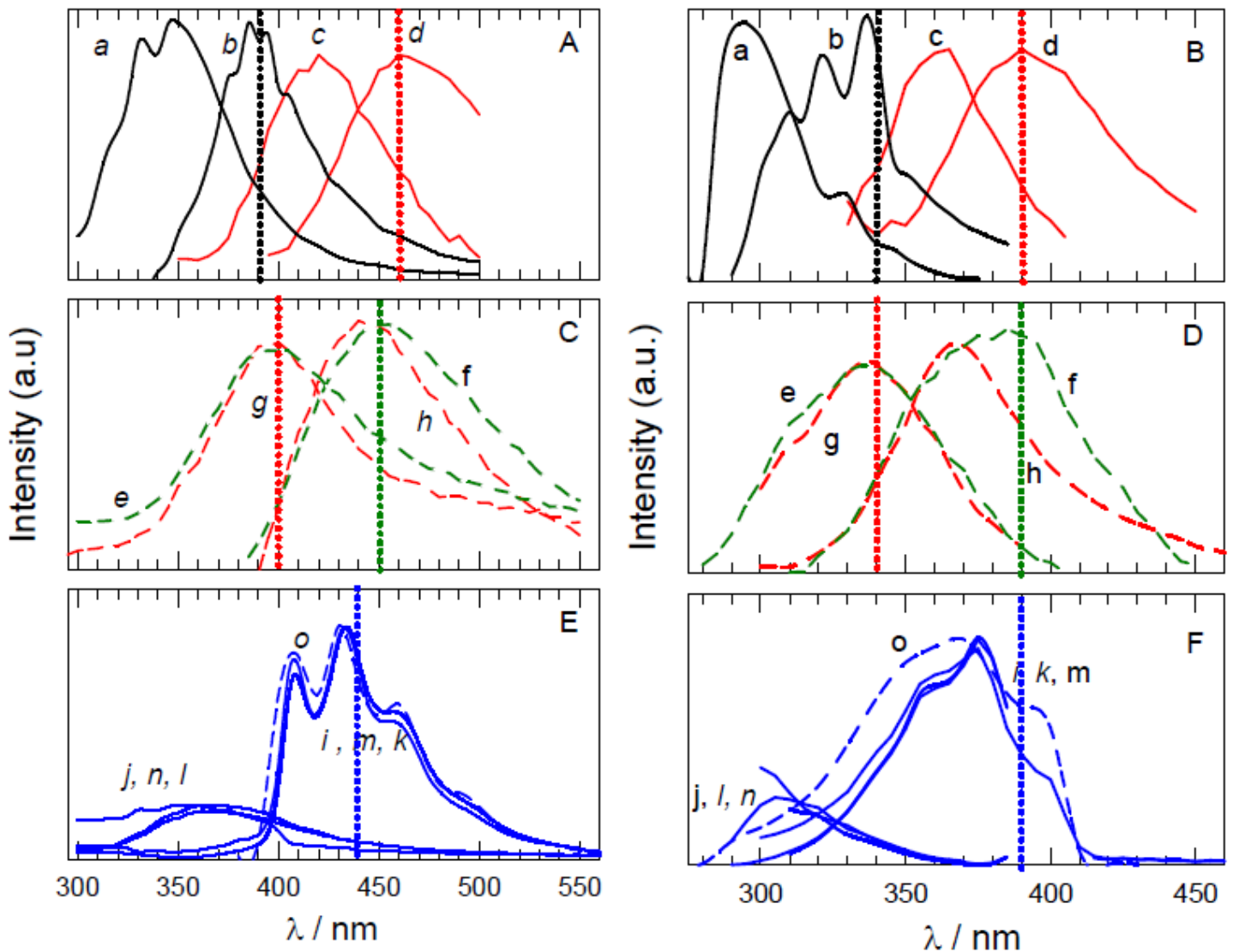

Figura 4.5. Espectros de emisión $\mathrm{PL}(\mathrm{A}, \mathrm{C}$ y $\mathrm{E})$ y excitación $\mathrm{PLE}(\mathrm{B}, \mathrm{D}$ y $\mathrm{F}) \mathrm{PL}$ de las especies contribuyentes obtenidas por análisis multilineal de las EEMs medidas a $313 \mathrm{~K}$ de suspensiones en tolueno saturadas en Ar de TD Si nps (a y b, líneas sólidas negras), PATD Si nps (c y d, líneas sólidas rojas), BU Si nps (e y f, líneas discontinuas verdes), PA-BU Si nps ( $\mathrm{g} \mathrm{h}$, líneas rojas discontinuas), SiOx-TD Si nps (i y j, líneas azules discontinuas), PASiOx-TD Si nps ( $\mathrm{y}$ l, líneas azules sólidas), MASiOx-TD’ Si nps ( $m$ y n, líneas sólidas azules) y PASiOx-BU Si nps (o, línea azul punteada). Las líneas verticales representan las longitudes de onda de excitación

(B, D y F) y detección ( $A, C$ y $E$ ) para los experimentos resueltos en el tiempo.

Se midieron los tiempos de vida (T) y rendimientos cuánticos de PL ( $\left.\Phi_{\mathrm{PL}}\right)$ a $298 \mathrm{~K}$ a longitudes de onda de excitación y emisión específicas a fin de excitar y/o detectar selectivamente alguna de las especies contribuyentes para cada Si np estudiada. Los t medidos para TD Si nps, BU Si nps y sus congéneres con superficies funcionalizadas resultaron entre 1 y $7 \mathrm{~ns}$, como se muestra en la tabla 4.2. En condiciones de bajas fluencias de excitación, los estados excitados decaen unimolecularmente, con lo cual vale la relación $\Phi_{\mathrm{em}}=\mathrm{k}_{\mathrm{r}}$ $I\left(k_{r}+\sum i k_{n r, i}\right)$, siendo $k_{r}$ la constante de velocidad para el decaimiento radiativo y $\sum i k_{n r, i}$ la suma de constantes de velocidad para los canales no radiativos. Por definición vale $\mathrm{T}=1 /\left(\mathrm{k}_{\mathrm{r}}+\sum \mathrm{k}_{\mathrm{nr}, \mathrm{i}}\right)$, con lo cual del producto $\mathrm{T}^{\star} \Phi_{\mathrm{em}, 298}$ $\mathrm{k}$ se obtiene $\mathrm{k}_{\mathrm{r}}$. Considerando los datos en la tabla 4.2, los valores de $\mathrm{k}_{\mathrm{r}}$ están en el intervalo $(1-60)^{*} 10^{7} \mathrm{~s}^{-1}$. Como se discute en los párrafos siguientes, al considerar partículas no emisivas al momento de estimar $\Phi_{\mathrm{PL}}$ los valores obtenidos para $\mathrm{k}_{\mathrm{r}}$ son límites inferiores. 


\begin{tabular}{|c|c|c|c|c|c|c|}
\hline Si np & Emisor & $\mathrm{EG}( \pm 0.05)(\mathrm{eV})$ & $\begin{array}{c}\lambda_{\mathrm{em}}^{\max } \\
(\mathrm{eV})\end{array}$ & $\begin{array}{c}\text { SS }( \pm 0.05) \\
(\mathrm{eV})\end{array}$ & т (\%) (ns) & $\Phi_{\mathrm{PL}}( \pm 0.05)$ \\
\hline \multirow{2}{*}{ TDSiNPs } & $a$ & 3.4 & 3.4 & 0.67 & \multirow{2}{*}{$\begin{array}{c}0.5(76) \\
3.15(19)\end{array}$} & 0.2 (aire 0.005 ) \\
\hline & $b$ & 3.15 & 3.1 & 0.53 & & 0.19 \\
\hline \multirow{2}{*}{ PA-TDSiNPs } & $c$ & 3.0 & 2.95 & 0.49 & \multirow{2}{*}{$\begin{array}{l}1.6 \pm 0.1(70) \\
4.3 \pm 0.1(27)\end{array}$} & 0.16 \\
\hline & $d$ & 2.7 & 2.7 & 0.50 & & 0.2 (aire 0.14 ) \\
\hline \multirow{2}{*}{$\mathrm{SiO}_{\mathrm{x}}$-TDSiNPs } & $i$ & 3.0 & 3.0 & 0.45 & $1.27 \pm 0.05$ & 0.13 \\
\hline & $j$ & 3.4 & 3.5 & 0.59 & ---- & ---- \\
\hline \multirow{2}{*}{ PA-SiOx-TDSiNPs } & k & 3.0 & 3.0 & 0.45 & $1.30 \pm 0.05$ & 0.36 \\
\hline & 1 & 3.4 & 3.4 & 0.60 & --- & 0.3 \\
\hline \multirow{2}{*}{ MASiO $_{x}$-TD'SiNPsa } & $m$ & 3.0 & 2.85 & 0.43 & $1.19 \pm 0.05$ & 0.76 (aire 0.44 ) \\
\hline & $n$ & 3.3 & 3.4 & 0.62 & --- & ---- \\
\hline \multirow{2}{*}{ BUSiNPs } & e & 3.1 & 3.1 & 0.55 & $1.5 \pm 0.5$ & ---- \\
\hline & $f$ & 2.8 & 2.8 & 0.45 & $7 \pm 0.5$ & ---- \\
\hline \multirow{2}{*}{ PA-BUSiNPs } & $g$ & 3.15 & 3.1 & 0.56 & \multirow{2}{*}{$\begin{array}{l}1.2 \pm 0.3(80) \\
4.5 \pm 0.1(20)\end{array}$} & 0.25 \\
\hline & $h$ & 2.65 & 2.75 & 0.63 & & 0.34 \\
\hline $\mathrm{SiO}_{\mathrm{x}-\mathrm{BUSiNPs}}{ }^{\mathrm{a}}$ & & 3.0 & 2.85 & 0.45 & $1.6 \pm 0.05$ & ---- \\
\hline PASiOx-BUSiNPs & 0 & 2.95 & 2.85 & 0.44 & $1.1 \pm 0.1$ & 0.1 \\
\hline
\end{tabular}

Tabla 4.2. EG, máximo de emisión ( $\lambda_{e m, m a x}$ ), corrimiento de Stokes (SS), tiempos de decaimiento (T) y rendimientos cuánticos de PL $\left(\Phi_{\mathrm{em}}\right)$ de las distintas especies emisoras, medidos a $298 \mathrm{~K}$ en atmósfera de Ar. Como se detalla en el texto, los valores mencionados son límites inferiores. En gris están resaltados los resultados para partículas con superficies oxidadas. a Datos de referencia [16].

A partir del umbral de excitación del espectro de excitación para cada especie individual pudo evaluarse el EG de los portadores de carga en las Si nps preparadas [16][34]. Los valores calculados se muestran en la tabla 4.2, y están de acuerdo con los valores para los máximos de emisión, lo cual sugiere que los procesos de emisión y excitación pueden originarse en la misma transición. Los SS correspondientes, que también se presentan en dicha tabla, varían entre 0.4 y $0.6 \mathrm{eV}$.

Los espectros de PL de suspensiones de TD Si nps muestran la contribución de dos especies (a y b en las figuras 4.5. A y B). Estas bandas anchas (FHWM $\sim \Delta E=400 \mathrm{meV}$ ) se extienden en el rango de 350 a $450 \mathrm{~nm}$ y junto con los tiempos de decaimiento en el régimen de los ns están de acuerdo con los reportados para $\mathrm{Si}$ nps de $1 \mathrm{~nm}$ de diámetro [8][9][35]. Asumiendo que la PL detectada está controlada por confinamiento cuántico, pueden estimarse tamaños de $1.3-1.6 \mathrm{~nm}$ a partir de correlaciones teóricas entre EG y el diámetro para Si nps cristalinas terminadas en $\mathrm{H}$ [7], en concordancia con los valores determinados.

Los espectros de PL de PA-TD Si nps también muestran la contribución de dos especies (c y d en las figuras 4.5. A y B) con PL corridas al rojo con respecto a a y b. Debido a que PA-TD Si nps y TD Si nps difieren mayormente en su composición superficial (grupos $\quad: \mathrm{Si}_{-} \mathrm{CH}_{2}-\mathrm{CH}_{2}-\mathrm{CH}_{2}-\mathrm{NH}_{2} \mathrm{O} \quad \mathrm{Si}-\mathrm{NH}-\mathrm{CH}_{2}-\mathrm{CH}=\mathrm{CH}_{2}$ en lugar de

绢-H), las diferencias observadas en las EEMs solo pueden ser atribuidas a una fuerte dependencia de la PL con la composición química de la superficie. Trabajos recientes, tanto teóricos como experimentales, han indicado que la cobertura de Si nps con grupos alquilo (Si-C) resultan en un cambio mínimo en el espectro de 
PL. Debido a que Si nps con terminaciones Si-N exhiben corrimientos significativos en su PL comparada a la de Si nps con $\mathrm{H}$ terminales [27][36], los cambios observados pueden atribuirse a enlaces Si-N.

Por otro lado, los espectros de emisión y excitación de las partículas amorfas BU Si nps (e y f en la figura 4.5. C y D) están corridos al rojo con respecto a las cristalinas TD Si nps, lo cual es esperable a partir de cálculos teóricos para partículas cristalinas y amorfas de tamaños similares [37][7]. Se reportó recientemente [27] que Si nps $\mathrm{H}$ terminales expuestas al surfactante $\mathrm{TOABr}$ desarrollan un espectro de $\mathrm{PL}$ en la región de 400 a 450 $\mathrm{nm}$ como consecuencia de la formación de enlaces Si-N superficiales. Debido a la falta de evidencia aportada por XPS y FITR sobre la formación de entornos Si-N en BU Si nps [16], la quimisorción de TOABr a la superficie de las partículas no es considerada en las condiciones de síntesis empleadas. De hecho, la PL de las especies e y f es coincidente con lo reportado para Si nps de $1.5 \mathrm{~nm}$ obtenidas por grabado electroquímico de films $\mathrm{pSi}$ [38]. La EEM de PA-BU Si nps muestra la contribución de dos especies emisoras ( $g$ y h en 4.5. C y D) con espectros de excitación y emisión similares a los de las especies e y f. Por lo tanto, entornos de Si-C y Si-N confinando las BU Si nps no parecen afectar en forma significativa la densidad de estados dentro del volumen de captura de los portadores de carga espacialmente confinados, lo cual conlleva a observar PL en Si nps amorfas [39].

Sin embargo, Si nps sintetizadas por distintas vías (estrategias TD o BU) pero oxidadas en su superficie y con distintos grupos en la misma (SiOx-TD Si nps, PASiOx-TD Si nps, SiOx-TD' Si nps, MASiOx-TD' Si nps, SiOxBU Si nps y PASiOx-BU Si nps) muestran EEMs (Figura 4.5. E y F) y tiempos de decaimiento de PL similares (Ver tabla 4.2). Estas observaciones indican fuertemente que la distribución espectral de las EEMs y los tiempos de decaimiento de PL de Si nps oxidadas con diámetros menores a $3 \mathrm{~nm}$ presentando estructuras superficiales

汭-O-Si $=$ NO dependen de la estructura cristalina, del tamaño o de la cobertura orgánica. La presencia de estructuras $\quad$ Si-O-Si $i$ genera una reducción de EG en el orden de $0.2-0.4 \mathrm{eV}$, con respecto a TD Si nps pasivadas con $\mathrm{H}$. Estas observaciones están de acuerdo con estudios teóricos que predicen que la adición de átomos de oxígeno a la superficie de clusters de $\mathrm{Si} \mathrm{H}$ terminales de $1 \mathrm{~nm}$ de diámetro reducen el gap óptico en fracciones de eV en caso de configuraciones puente ( $\mathrm{Si}-\mathrm{O}-\mathrm{Si}$ ), pero produciendo efectos en el orden de los eV en configuraciones que impliquen dobles ligaduras $(>\mathrm{Si}=0)[8][10]$.

Los espectros de excitación y emisión de Si nps oxidadas presentan estructura con una separación de picos de 140-180 meV, en el orden reportado para desdoblamientos debido a progresiones vibracionales en experimentos de moléculas únicas realizados con Si nps cristalinas y oxidadas embebidas en una matriz de $\mathrm{SiO}_{2}$ [40][41][42][43]. La diferencia de energía cercana a $150 \mathrm{meV}$ puede atribuirse a fonones longitudinales ópticos (LO) y transversales ópticos (TO) asociados a estructuras Si-O-Si presentes en la superficie [41]. Asumiendo interacciones electrón-fonón, la presencia a mayores energías de la línea vibracional 00 observada en este trabajo $(>3.0 \mathrm{eV}$ ) puede correlacionarse con el pequeño tamaño del núcleo de $\mathrm{Si}$ en partículas en régimen de confinamiento cuántico. Sin embargo, esta idea no apoya la alta localización del estado excitado en la interfase Si/SiOx, lo cual está apoyado en la independencia de la cristalinidad o tamaño de partícula observada para los espectros de PL y tiempos de vida [42]. Debido a que el efecto generado por el esfuerzo compresivo de la matriz sobre los nanocristales puede influenciar la PL de Si nps [44], cualquier discusión sobre el origen de las diferencias observadas requiere medidas de moléculas únicas con Si nps ultra-pequeñas que emiten en el azul.

En conclusión, los resultados obtenidos indican que para estructuras pequeñas donde el número de átomos superficiales representa una fracción del número total de átomos (para Si nps cristalinas de $1.3 \mathrm{~nm}$ la fracción es ca. 70\%), diferentes adsorbatos y arreglos atómicos llevan a distintos estados electrónicos superficiales que influencian la PL de Si nps. Variaciones en los corrimientos de Stokes en Si nps cristalinas en general se atribuyen a niveles de energía debajo del mínimo de la banda de conducción, lo cual lleva al atrapamiento del estado excitado en estados altamente localizados. Se reportó que dichos niveles son introducidos por estados superficiales, electrones desapareados formando "enlaces colgantes" o por interacciones electrón-fonón [45]. Es interesante notar en la figura 4.6 (A) una correlación aceptable entre los corrimientos de Stokes y los BG determinados para distintas especies emisoras. Estas observaciones junto al hecho de que las mismas 
transiciones están vinculadas a los procesos de absorción y emisión, dan un sustento fuerte a la contribución de estados superficiales a niveles energéticos que participan del proceso de emisión. Es importante notar que las especies $d$ y $h$ que contribuyen en el caso de PA-TD Si nps y PA-BU Si nps, respectivamente, son excepciones. Átomos de $\mathrm{N}$ quimisorbido pueden introducir niveles adicionales por debajo del mínimo de la banda de conducción que no afectan a BG. De todos modos, la determinación precisa de la naturaleza de las especies contribuyentes no es posible de forma unívoca y los datos pueden interpretarse de distintos modos.

En el caso de Si nps de tamaños mayores a $2 \mathrm{~nm}$ estudiadas en literatura que muestran emisión desde el verde al IR cercano, originadas por efectos claros de confinamiento cuántico [44][46][47], también se reportaron cambios importantes en la PL luego de modificar la química superficial de las nanopartículas [48][49][50]. Teniendo esto en cuenta, la participación de estados superficiales en el proceso de emisión en Si nps se manifiesta también en sistemas son relaciones superficie/volumen aún menor.
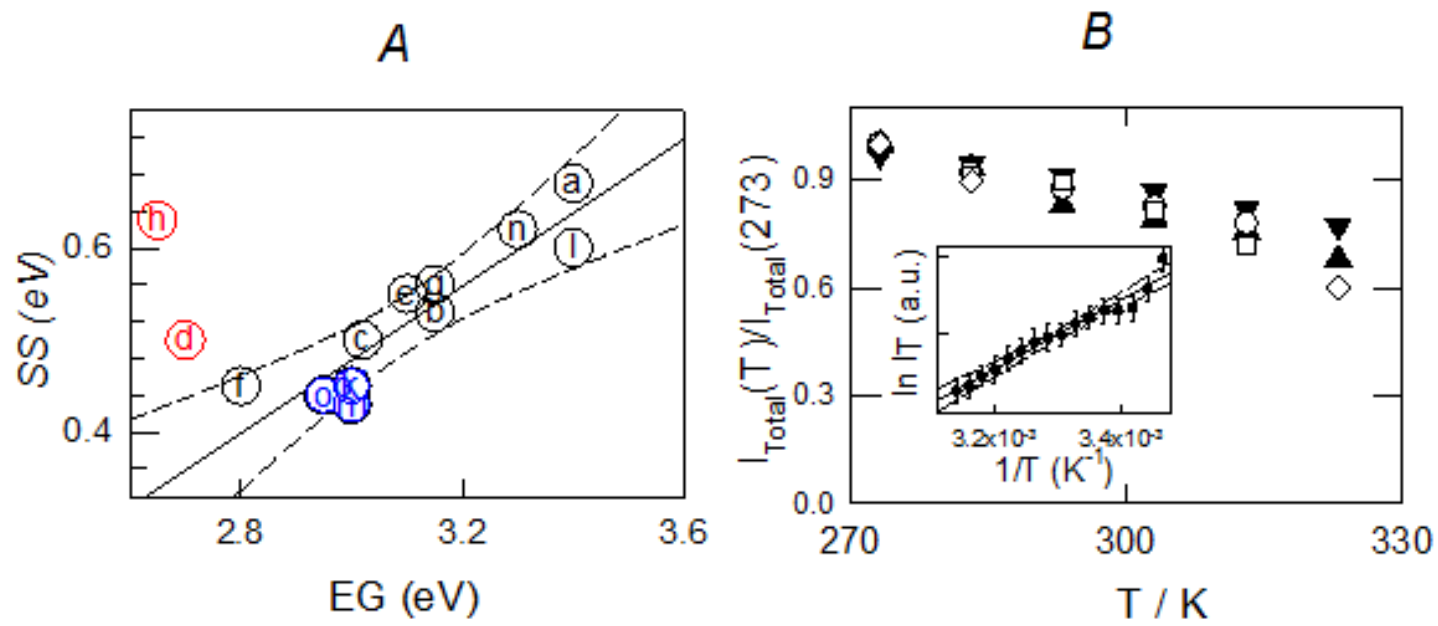

Figura 4.6. (A) Corrimientos de Stokes en función de BG. Las letras dentro de los círculos corresponden a las distintas especies emisoras en cada sistema estudiado. (B) Cociente entre la intensidad total de PL a una temperatura dada con respecto a la determinada a $273 \mathrm{~K}(\mathrm{IT}(\mathrm{T}) / \mathrm{IT}(273))$ para suspensiones saturadas en Ar. Se presentan los resultados para las a $(\boldsymbol{\nabla}), \mathrm{b}(\mathbf{\Delta}), \mathrm{c}(\diamond), \mathrm{k}$ (O), and $\mathrm{m}(\square)$ vs. T. En el gáfico insertado se representa In IT vs $\mathrm{T}^{-1}$ para la especie $\mathrm{k}$.

\subsubsection{Efecto de la temperatura sobre $\Phi_{\mathrm{PL}}$}

El rendimiento cuántico global de PL (considerando la totalidad del espectro de emisión, incluyendo todas las especies emisoras) de las $\mathrm{Si}$ nps en suspensión de tolueno saturadas en Ar disminuye al aumentar la temperatura. Los valores de $\Phi_{P L}$ se recuperan al volver a la temperatura inicial, lo cual sugiere un mecanismo de quenching reversible dependiente de la temperatura. Si se representa $\ln \left(\Phi_{\mathrm{PL}}{ }^{-1}-1\right) \mathrm{vs}$. $^{-1}$ se observa una $^{-1}$ curvatura en los gráficos, lo cual es esperable si dos o más procesos de desactivación con energías de activación comparables están presentes. De hecho los valores de $\Phi_{\mathrm{PL}}$ no pueden separar las contribuciones individuales de cada especie emisora. A fin de comprender el efecto de la temperatura sobre la PL se realizó un análisis PARAFAC sobre las EEMs obtenidas para cada Si np estudiada a las distintas temperaturas ensayadas, desde 273 a $330 \mathrm{~K}$. Este análisis muestra que la intensidad, pero no los valores de EG o los corrimientos de Stokes de cada componente individual depende de la temperatura. La figura 4.6 (B) muestra la disminución de $\mathrm{I}_{T} / \mathrm{I}_{273}$ con la temperatura para distintas especies contribuyentes. Se empleó dicho cociente para compensar todos los parámetros no dependientes de la temperatura que afectan $I_{T}$ como la respuesta del equipo o la concentración de partículas. Salvo para la especie d, todos los valores determinados de $I_{T} / I_{273}$ caen alrededor de la misma curva, lo cual indica que el proceso de quenching térmicamente activado no se correlaciona con la estructura cristalina o la química superficial de las partículas estudiadas. Por otro lado, es 
interesante mencionar que los valores de т no mostraron variaciones, dentro del error experimental, en el intervalo de temperaturas de 200 a $330 \mathrm{~K}$.

El modelo de Calcott assume un equilibrio térmico entre dos estados de distinta multiplicidad y constante de decaimiento radiativo, separados por una barrera energética $\Delta \mathrm{E}$, y es usado ampliamente para interpretar la dependencia con la temperatura para constantes radiativas $\mathrm{k}_{\mathrm{r}}$ [13][14][51][52][53]. A muy bajas temperaturas, todos los excitones se encuentran atrapados en el estado inferior mientras que a mayores temperaturas, el estado superior es poblado, siendo este último el estado luminiscente. Valores típicos de $\Delta \mathrm{E}$ están en el orden de $3 \mathrm{meV}$ [13][52]. Considerando que no se espera que kr varíe con la temperatura para T>200 K, la disminución en la intensidad de PL al aumentar la temperatura se atribuyó al rol dominante de canales de recombinación no radiativos [13][54]. Hay dos modelos que se emplean mayoritariamente al momento de estudiar estos procesos: Uno de comportamiento normal de tipo Arrhenius [13][55], y la ionización del estado excitado por efecto túnel a través de una barrera de potencial, con una dependencia con T de tipo Berthelot [54[][55][56][57][58]. Sin embargo, considerando que nuestros resultados muestran que $\left(\mathrm{k}_{\mathrm{r}}+\sum \mathrm{k}_{\mathrm{nr}, \mathrm{i}}\right)=\mathrm{T}^{-1}$ permanece constante con la temperatura, siendo $k_{r}$ independiente de $T$, es esperable que los procesos no radiativos competitivos $\sum k_{n r, i}$ originados en el mismo estado excitado sean también independientes de la temperatura. Por lo tanto, el uso de cualquiera de los mecanismos mencionados no explica satisfactoriamente la dependencia de la intensidad de $\mathrm{PL}$ con la temperatura observada.

A partir de estas consideraciones, puede postularse un mecanismo reversible de quenching estático térmico. Un aumento en la temperatura conduce a un aumento en la población de partículas no emisoras, el cual puede revertirse al disminuir nuevamente la temperatura. Más aun, teniendo en cuenta la falta de correlación entre la constante de quenching con la química superficial de las partículas, es esperable que la estructura de Si este relacionada con la generación de estados oscuros (no emisivos). Estas observaciones están de acuerdo con las realizadas por Diener y colaboradores [59], en las cuales la excitación en el IR de pSi resulta en una caída en la intensidad de PL pero con solo un pequeño cambio en el tiempo de vida de PL. Estos autores sugirieron que la excitación en el IR creaba enlaces colgantes adicionales o habilitaba nuevos canales no radiativos, lo cual aumentaba la fracción de nanocristales oscuros.

Los gráficos de In $\mathrm{I}_{\mathrm{T}} \mathrm{vs} \mathrm{T}^{-1}$ (inserto en la figura $4.6 \mathrm{~B}$ ) dan líneas rectas cuyas pendientes dan un cambio de entalpía $\Delta \mathrm{H}=+(15 \pm 1) \mathrm{meV} / \mathrm{mol}$, para el proceso térmico que lleva a la generación de conformaciones no emisivas. El cambio de energía entre configuraciones oscuras y brillantes es muy pequeño para ser asignado a la generación de enlaces colgantes, con lo cual se sugirió que el proceso en estudio consta de pequeños cambios conformacionales.

Diversos experimentos en bibliografía relacionan la existencia de fracciones oscuras en puntos cuánticos con la dinámica de parpadeo [60]. A través de estudios de PL de moléculas individuales se observó que el parpadeo de PL está en el orden de los milisegundos, y que el tiempo de parpadeo escala con la potencia del laser empleado para la excitación. A su vez, si los estudios se extienden por horas se observó un fotoblanqueo que se asignó a desorden en la red luego de la excitación prolongada. [61].

La generación foto-asistida de partículas oscuras implicaría la reducción de la intensidad de señal de PL en estado estacionario en función del tiempo de irradiación. Debido a que no se observaron cambios en la intensidad de PL en estado estacionario con el tiempo de iluminación para las partículas sintetizadas en este trabajo, configuraciones oscuras de Si nps pueden asumirse en equilibrio térmico con configuraciones brillantes previo a la iluminación. La figura 4.7 ilustra el proceso térmico entre partículas oscuras y brillantes que lleva al aumento de la fracción oscura al aumentar la temperatura. Si una estadística de parpadeo del tipo potencial fuera válida para las Si nps estudiadas, esta podría surgir de la existencia de una multiplicidad de configuraciones oscuras (o brillantes) con distintas barrreras energéticas. 


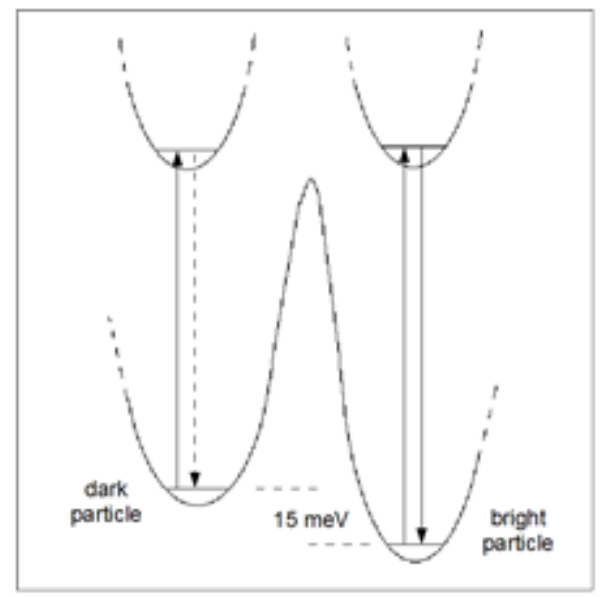

Figura 4.7. Representación del proceso térmico involucrado en la interconversion de partículas oscuras y brillantes (no escalado). Las flechas representan procesos radiativos (línea continua) y no radiativos (línea discontinua).

\subsection{Conclusión}

La dependencia de la PL con el tamaño, la cristalinidad, la estructura y química superficial para Si nps constituye un desafío importante al momento de diseñar sensores y fotosensibilizadores. A su vez, la baja estabilidad de Si nps $\mathrm{H}$ terminales conlleva a procesos de oxidación no deseados, a menos que los procesos de síntesis y modificación sean realizados en estrictas condiciones de ausencia de oxígeno. Nuestros resultados muestran que las propiedades de PL de Si nps de $1-2 \mathrm{~nm}$ oxidadas de forma controlada que presentan estructuras del tipo Si-O-Si son independientes de la estrategia sintética (TD o BU) y de la derivatización post-sintética con moléculas orgánicas con distintos grupos funcionales. Esta observación tiene importantes consecuencias para el desarrollo de usos tecnológicos de las Si nps, ya que la ruta sintética a elegir puede seleccionarse a partir de costos y disponibilidad de reactivos o equipamiento. Incluso aunque las principales vías de síntesis requieren la minimización de oxígeno disuelto en el medio, el uso posterior de reacciones de silanización para generar una cobertura orgánica puede realizarse en atmósfera de aire y a temperatura ambiente. Como resultado, moléculas biocompatibles pueden ser unidas a la superficie sin cambios en las propiedades de PL. Estas últimas propiedades son importantes para la dirección específica a blancos y prevenir la opsonización en aplicaciones biológicas.

\subsection{Referencias}

[1] Llansola Portole『s, M. J.; Rodriguez Nieto, F.; Soria, D. B.; Amalvy, J. I.; Peruzzo, P. J.; Mårtire, D. O.; Kotler, M. n.; Holub, O.; Gonzalez, M. n. C. The Journal of Physical Chemistry C 2009, 113, 13694.

[2] Llansola Portole匹s, M. J.; David Gara, P. M.; Kotler, M. n. L.; Bertolotti, S.; San Romann, E.; Rodringuez, H. n. B.; Gonzalez, M. n. C. Langmuir 2010, 26, 10953.

[3] Popplewell, J. F.; King, S. J.; Day, J. P.; Ackrill, P.; Fifield, L. K.; Cresswell, R. G.; di Tada, M. L.; Liu, K. Journal of Inorganic Biochemistry 1998, 69, 177.

[4] Park, J.-H.; Gu, L.; von Maltzahn, G.; Ruoslahti, E.; Bhatia, S. N.; Sailor, M. J. Nat Mater 2009, 8, 331.

[5] Yu, M. K.; Park, J.; Jon, S. Theranostics 2012, 2, 42.

[6] Eckhoff, D. A.; Sutin, J. D. B.; Clegg, R. M.; Gratton, E.; Rogozhina, E. V.; Braun, P. V. The Journal of Physical Chemistry B 2005, 109, 19786.

[7] Delley, B.; Steigmeier, E. F. Applied Physics Letters 1995, 67, 2370.

[8] Draeger, E. W.; Grossman, J. C.; Williamson, A. J.; Galli, G. The Journal of Chemical Physics 2004, 120, 10807.

[9] Dai, Y.; Han, S.; Dai, D.; Zhang, Y.; Qi, Y. Solid State Communications 2003, 126, 103. 
[10] Puzder, A.; Williamson, A. J.; Grossman, J. C.; Galli, G. Journal of the American Chemical Society 2003, 125, 2786.

[11] Huang, W.-Q.; Huang, Z.-M.; Cheng, H.-Q.; Miao, X.-J.; Shu, Q.; Liu, S.-R.; Qin, C.-J. Applied Physics Letters 2012, 101, 171601.

[12] Takeoka, S.; Fujii, M.; Hayashi, S. Physical Review B 2000, 62, 16820.

[13] Orii, T.; Hirasawa, M.; Seto, T.; Aya, N.; Onari, S. Eur. Phys. J. D 2003, 24, 119.

[14] Lüttjohann, S.; Meier, C.; Offer, M.; Lorke, A.; Wiggers, H. EPL (Europhysics Letters) 2007, 79, 37002.

[15] Principles of Fluorescence Spectroscopy; Third ed.; Lakowicz, J. R., Ed.; Springer: Singapore, 2006.

[16] Llansola Portolés, M. J.; Pis Diez, R.; Dell'Arciprete, M. L.; Caregnato, P.; Romero, J. J.; Mártire, D. O.; Azzaroni, O.; Ceolín, M.; Gonzalez, M. C. The Journal of Physical Chemistry C 2012, 116, 11315.

[17] Warner, I. M.; Christian, G. D.; Davidson, E. R.; Callis, J. B. Anal. Chem. 1977, 49, 564.

[18] Li, Z. F.; Ruckenstein, E. Nano Letters 2004, 4, 1463.

[19] Caregnato, P.; Forbes, M. D. E.; Soria, D. B.; Maørtire, D. O.; Gonzalez, M. n. C. The Journal of Physical Chemistry C 2010, 114, 5080.

[20] Caregnato, P.; Dell'Arciprete, M. L.; Gonzalez, M. C. Photochemical \& Photobiological Sciences 2013.

[21] Huisken, F.; Hofmeister, H.; Kohn, B.; Laguna, M. A.; Paillard, V. Applied Surface Science 2000, 154$155,305$.

[22] Baldwin, R. K.; Pettigrew, K. A.; Ratai, E.; Augustine, M. P.; Kauzlarich, S. M. Chemical Communications 2002, 0, 1822.

[23] Launer, J. P. MRS Bulletin 1997, 22, 5.

[24] Wahab, M. A.; Kim, I.; Ha, C.-S. Journal of Solid State Chemistry 2004, 177, 3439.

[25] Soto, G.; Samano, E. C.; Machorro, R.; Cota, L., 1998; 1311.

[26] Silicon-Based Material and Devices; Nalwa, H. S., Ed.; Academic Press: San Diego, 2001.

[27] Dasog, M.; Yang, Z.; Regli, S.; Atkins, T. M.; Faramus, A.; Singh, M. P.; Muthuswamy, E.; Kauzlarich, S. M.; Tilley, R. D.; Veinot, J. G. C. ACS Nano 2013, 7, 2676.

[28] Zhou, S.; Liu, W.; Cai, C.; Liu, H. 2010, 79950T.

[29] Vandenberg, E. T.; Bertilsson, L.; Liedberg, B.; Uvdal, K.; Erlandsson, R.; Elwing, H.; Lundström, I. Journal of Colloid and Interface Science 1991, 147, 103.

[30] Naviroj, S.; Koenig, J. L.; Ishida, H. Journal of Macromolecular Science, Part B 1983, 22, 291.

[31] Wang, P. W.; Bater, S.; Zhang, L. P.; Ascherl, M.; Craig Jr, J. H. Applied Surface Science 1995, 90, 413.

[32] Ouyang, M.; Yuan, C.; Muisener, R. J.; Boulares, A.; Koberstein, J. T. Chemistry of Materials 2000, 12, 1591.

[33] Kluth, G. J.; Sung, M. M.; Maboudian, R. Langmuir 1997, 13, 3775.

[34] Garrido, B.; Lopez, M.; Gonzalez, O.; Perez-Rodriguez, A.; Morante, J. R.; Bonafos, C. Applied Physics Letters 2000, 77, 3143.

[35] Smith, A.; Yamani, Z. H.; Roberts, N.; Turner, J.; Habbal, S. R.; Granick, S.; Nayfeh, M. H. Physical Review B 2005, 72, 205307.

[36] Tu, C.-C.; Hoo, J.-H.; Böhringer, K. F.; Lin, L. Y.; Cao, G. Opt. Lett. 2012, 37, 4771.

[37] Park, N.-M.; Choi, C.-J.; Seong, T.-Y.; Park, S.-J. Physical Review Letters 2001, 86, 1355.

[38] Xie, Y.; Wu, X. L.; Qiu, T.; Chu, P. K.; Siu, G. G. Journal of Crystal Growth 2007, 304, 476.

[39] Estes, M. J.; Moddel, G. Physical Review B 1996, 54, 14633.

[40] Saar, A. Journal of Nanophotonics 2009, 3, 032501.

[41] Martin, J.; Cichos, F.; Huisken, F.; von Borczyskowski, C. Nano Lett. 2008, 8, 656.

[42] El-Kork, N.; Huisken, F.; Borczyskowski, C. v. J. Appl. Phys. 2011, 110, 074312.

[43] Schmidt, T.; Chizhik, A. I.; Chizhik, A. M.; Potrick, K.; Meixner, A. J.; Huisken, F. Physical Review B 2012, 86, 125302.

[44] Kusova, K.; OndiC, L.; Klimesova, E.; Herynkova, K.; Pelant, I.; Danis, S.; Valenta, J.; Gallart, M.; Ziegler, M.; Honerlage, B.; Gilliot, P. Appl. Phys. Lett. 2012, 101, 143101.

[45] Pan, L.; Sun, Z.; Sun, C. Scripta Materialia 2009, 60, 1105.

[46] Meier, C.; Gondorf, A.; Luttjohann, S.; Lorke, A.; Wiggers, H. J. Appl. Phys. 2007, 101, 103112. 
[47] Hessel, C. M.; Henderson, E. J.; Veinot, J. G. C. Chem. Mater. 2006, 18, 6139.

[48] Hua, F.; Erogbogbo, F.; Swihart, M. T.; Ruckenstein, E. Langmuir 2006, 22, 4363.

[49] Li, X.; He, Y.; Swihart, M. T. Langmuir 2004, 20, 4720.

[50] Dasog, M.; Veinot, J. G. C. physica status solidi (a) 2012, 209, 1843.

[51] Calcott, P. D. J.; Nash, K. J.; Canham, L. T.; Kane, M. J.; Brumhead, D. Journal of Physics: Condensed Matter 1993, 5, L91.

[52] Brongersma, M. L.; Kik, P. G.; Polman, A.; Min, K. S.; Atwater, H. A. Applied Physics Letters 2000, 76, 351.

[53] Cha, D.; Shin, J. H.; Song, I.-H.; Han, M.-K. Applied Physics Letters 2004, 84, 1287.

[54] Chao, Y.; Houlton, A.; Horrocks, B. R.; Hunt, M. R. C.; Poolton, N. R. J.; Yang, J.; Siller, L. Applied Physics Letters 2006, 88, 263119.

[55] Street, R. A. Philosophical Magazine Part B 1978, 37, 35.

[56] Kapoor, M.; Singh, V. A.; Johri, G. K. Physical Review B 2000, 61, 1941.

[57] Rinnert, H.; Vergnat, M. Physica E: Low-dimensional Systems and Nanostructures 2003, 16, 382.

[58] Chao, Y. In Comprehensive Nanoscience and Technology; Editors-in-Chief: David, L. A., Gregory, D. S., Gary, P. W., Eds.; Academic Press: Amsterdam, 2011.

[59] Diener, J.; Ben-Chorin, M.; Kovalev, D. I.; Ganichev, S. D.; Koch, F. Thin Solid Films 1996, 276, 116.

[60] Durisic, N.; Wiseman, P. W.; Grütter, P.; Heyes, C. D. ACS Nano 2009, 3, 1167.

[61] Martin, J.; Cichos, F.; Chan, I. Y.; Huisken, F.; Borczyskowski, C. V. Israel Journal of Chemistry 2004, $44,341$. 
- 95 - 


\section{Capítulo 5: Fotofísica de Si nps: El efecto del tamaño}

\subsection{Introducción}

En el presente capítulo se discuten las propiedades ópticas de Si nps de distintos tamaños, sintetizadas por oxidación de fases de Zintl. Variando la cantidad de precursor de Si y oxidante empleados, pudieron obtenerse Si nps de distintos tamaños. Posteriormente fueron cubiertas con 2-propenilamina usando reacciones de hidrosililación térmica, y luego se estudiaron sus propiedades ópticas y electroquímicas en función del tamaño. Se encontró que la variación del tamaño influye en la posición del umbral de absorción, máximo de emisión y potencial de la banda de conducción, siendo estas propiedades de fundamental interés al momento de diseñar díadas que soporten procesos de transferencia de energía o carga.

\subsection{Aspectos sintéticos y metodológicos}

Los detalles referidos a síntesis y métodos experimentales han sido desarrollados en mayor extensión en los capítulos 2 y 3 . A continuación, se comentarán de forma breve los procedimientos relacionados al presente capítulo.

Las Si nps estudiadas en este capítulo fueron sintetizadas usando una adaptación de las técnicas publicadas por Kauzlarich y colaboradores [1][2][3]. Los detalles correspondientes a esta síntesis se encuentran en las secciones 3.2.4 a 3.2.7. Se emplearon reacciones de oxidación de fases de Zintl donde el Si sea un constituyente. En bibliografía se ha explorado el uso tanto de distintas fases de Zintl como de oxidantes de distinta fuerza. Una vez elegido el par redox a emplear, la variación de temperatura a lo largo de la reacción 0 la relación de precursor de Si a oxidante $\left(n_{s i}: n_{0 x}\right)$ puede emplearse para controlar el tamaño de las Si nps obtenidas. En este trabajo de tesis se utilizó como par redox $\mathrm{Mg}_{2} \mathrm{Si}$ y $\mathrm{NH}_{4} \mathrm{Cl}$, y la reacción empleada fue la siguiente:

$$
\mathrm{Mg}_{2} \mathrm{Si}+4 \mathrm{NH}_{4} \mathrm{Cl} \rightarrow 4 \mathrm{NH}_{3}+2 \mathrm{H}_{2}+2 \mathrm{MgCl}_{2}+\mathrm{Si}
$$

En un balón con 3 bocas se colocaron $120.00 \mathrm{ml}$ de DMF seco y $100.0 \mathrm{mg} \mathrm{de} \mathrm{Mg}_{2} \mathrm{Si}$ (1.3 mmol) en agitación. Esta solución fue calentada hasta $100 \mathrm{C}$ y purgada con $\mathrm{Ar}$ por 30 mintutos, y posteriormente se agregaron cantidades variables de $\mathrm{NH}_{4} \mathrm{Cl}$, a fin de variar la relación $n_{\text {si: }} n_{0 x}$ y se continuó el calentamiento y la purga, hasta que la mezcla entró en reflujo ( $153 \mathrm{C}$ ), luego de lo cual se cortó el burbujeo con Ar. En este punto, la mezcla se ve de color levemente azul, debido a partículas de $\mathrm{Mg}_{2} \mathrm{Si}$ en suspensión. La reacción procedió por $20 \mathrm{hs}$, y luego se enfrió lentamente. El avance de la reacción puede verificarse tomando una alícuota e iluminándola con luz de $350 \mathrm{~nm}$, para observar a ojo desnudo la emisión por parte de las Si nps. Al finalizar la reacción, la mezcla posee un color anaranjado. Esta mezcla se filtró usando membranas de $220 \mathrm{~nm}$ para remover el $\mathrm{Mg}_{2} \mathrm{Si}$ que no reaccionó, y luego se dializó contra solvente puro para remover los productos secundarios.

Las condiciones empleadas para la obtención de Si nps de distintos tamaños se detalla en la tabla 5.1.

\begin{tabular}{ccccccc}
\hline Muestra & $\mathrm{m}_{\text {Mg2Si }}(\mathbf{g})$ & $\mathrm{m}_{\text {NH4Cl }}(\mathbf{g})$ & $\mathbf{V}_{\text {DMF }}(\mathrm{ml})$ & $\mathbf{n}_{\mathrm{Si}}$ & $\mathbf{n}_{\text {ox }}$ & $\mathbf{n}_{\text {ox }} / \mathbf{n}_{\mathbf{S i}}$ \\
\hline S1 & 0.1 & 0.24 & 120 & $1.30 \mathrm{E}-03$ & $4.49 \mathrm{E}-03$ & 3.44 \\
S3 & 0.11 & 0.4 & 120 & $1.43 \mathrm{E}-03$ & $7.48 \mathrm{E}-03$ & 5.21 \\
S4 & 0.1 & 0.8 & 100 & $1.30 \mathrm{E}-03$ & $1.50 \mathrm{E}-02$ & 11.5
\end{tabular}

Tabla 5.1. Condiciones experimentales empleadas para la obtención de BU' Si nps. 
Para el paso de modificación superficial, se colocó en un balón de 3 bocas que contenía las cantidades adecuadas de Si nps en suspensión en DMF y del precursor orgánico según se indicó en 3.3 y $500 \mu$ de una solución de $\mathrm{H}_{2} \mathrm{PtCl}_{6} 5 \mathrm{mM}$ en $\mathrm{MeOH}$, completando con DMF hasta alcanzar un volumen de $120.00 \mathrm{ml}$. Luego la mezcla de reacción se calentó lentamente hasta llegar a reflujo. Simultáneamente se purgó profusamente la mezcla con Ar hasta el inicio del reflujo. La reacción se llevó a cabo durante ca. 20 hs, para luego retirar el calentamiento y enfriar lentamente. Finalmente, las Si nps se aislaron y purificaron por diálisis y ciclos de evaporación/disolución en el solvente de trabajo.

Al momento de analizar las ventajas y desventajas de este método resalta la facilidad para obtener cantidades importantes de Si nps, por lo cual fue el elegido para los experimentos que se describen en los capítulos siguientes, en los cuales la tarea sintética requirió grandes cantidades de reactivos.

\subsection{Resultados y discusión}

\subsubsection{Sintesis y caracterización}

Se sintetizaron Si nps por oxidación de $\mathrm{Mg}_{2} \mathrm{Si}$, y a partir del cambio en la relación de moles de oxidante y precursor de Si ( $n_{0 x}: n_{S i}$ ) se obtuvieron nanopartículas de tamaño controlado. Las distribuciones de tamaño obtenidas por análisis de micrografías TEM se muestran en la figura 5.2. Encontramos que al aumentar la cantidad de oxidante respecto del precursor de Si, el tamaño de las Si nps aumenta. El efecto de la condición de síntesis sobre el tamaño de las Si nps en esta vía de síntesis se muestra en la figura 5.1. Para corroborar la validez de esta vía de control realizamos un análisis de varianza (ANOVA) a nivel significación 0.05 observando que los tamaños medios poblacionales obtenidos son significativamente diferentes.

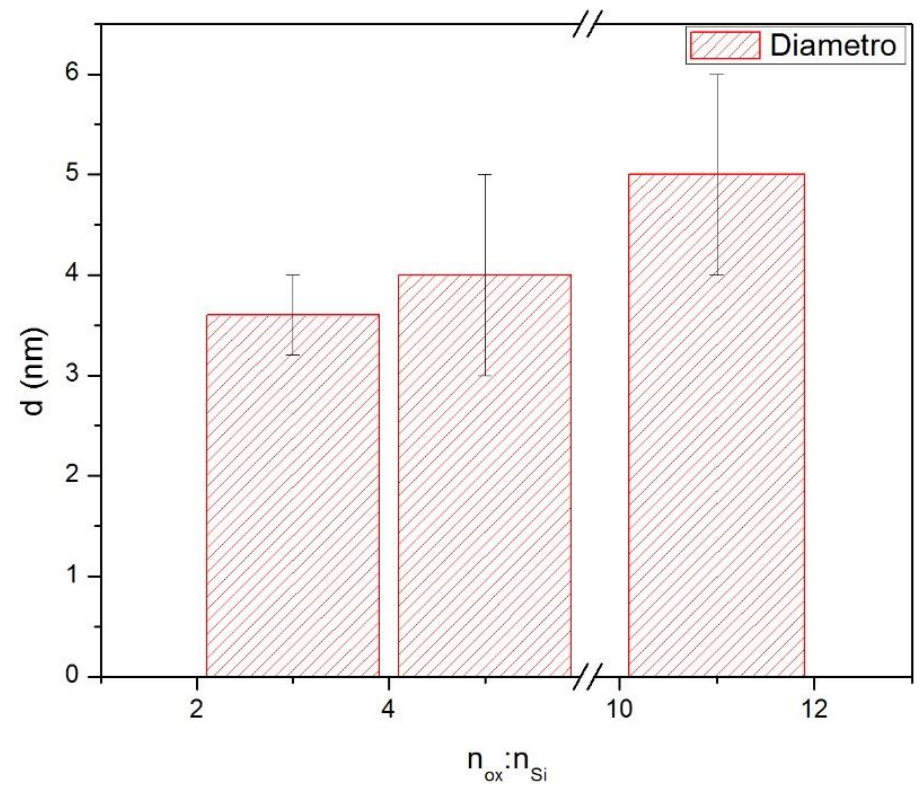

Figura 5.2. Efecto de la cantidad de oxidante sobre el tamaño de las Si nps sintetizadas en este capítulo. 
(a)

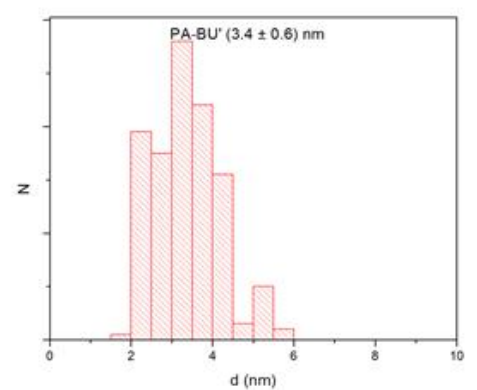

(b)

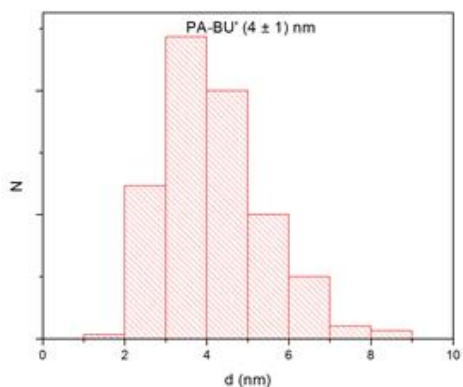

(c)

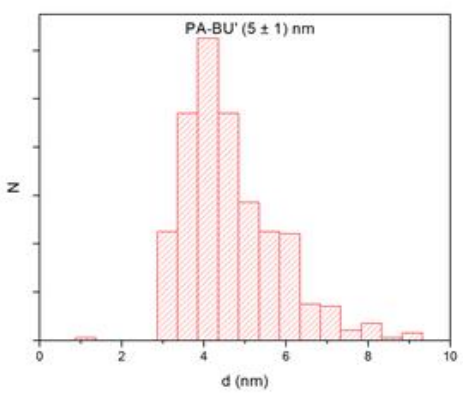

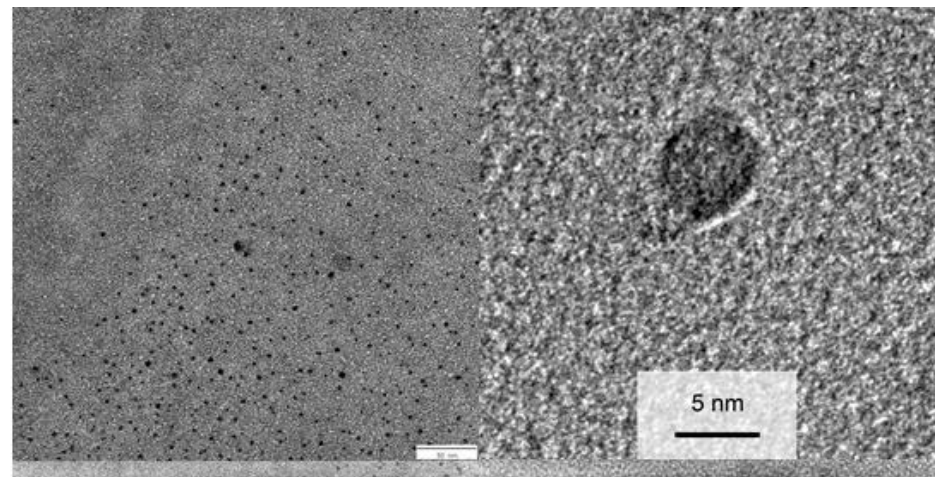
किता

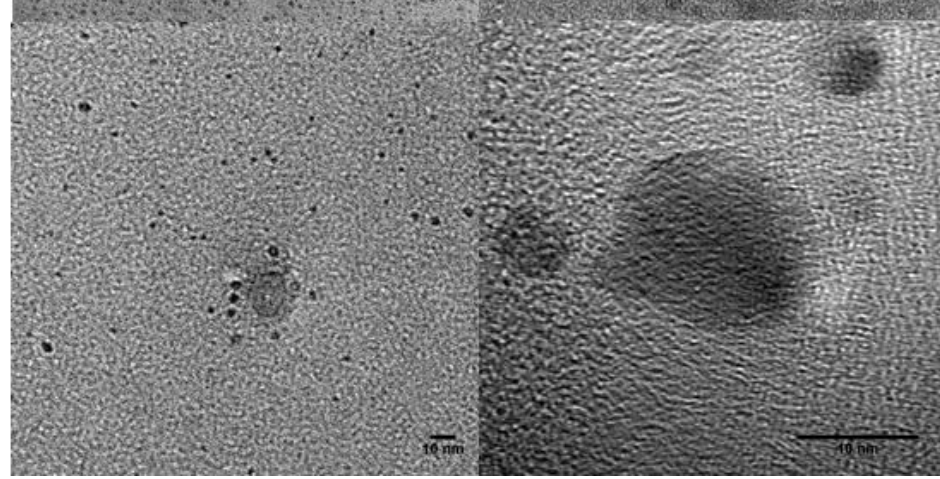

Figura 5.2. Distribuciones de tamaños, micrografías TEM representativas y micrografías HR-TEM de las distintas muestras analizadas en este capitulo. (a) PA-BU' Si nps de (3.4 \pm 0.6$) \mathrm{nm}$, (b) PA-BU' Si nps de (4 \pm 1$) \mathrm{nm}$ y (c) PA-BU' Si nps de (5 \pm 1$) \mathrm{nm}$.

Para entender el proceso involucrado y obtener un control preciso del tamaño del coloide se recurrió al modelo de nucleación en fase homogénea. El modelo clásico de formación de soles a partir de sistemas homogéneos implica la existencia de 2 procesos bien diferenciados: La nucleación y el crecimiento. Este fue desarrollado inicialmente por LaMer y colaboradores en 1950 [4], y se encuentra ampliamente documentado en la literatura. Básicamente, este propone que el proceso de formación de una nueva fase está regulado por contribuciones energéticas asociadas al seno de la fase formada (una contribución de volumen) y a la formación de una superficie nueva (contribución de superficie). La formación de una fase libera energía, mientras que la formación de una superficie nueva requiere energía. Estos efectos contrapuestos son los que dominan la evolución del sistema. Este comienza con un proceso de nucleación, donde los precursores reactivos forman pequeños agregados que son inestables desde el punto de vista termodinámico. Una vez que estos agregados alcanzan un tamaño crítico $r^{*}$, su crecimiento está favorecido y el crecimiento de la nueva fase se da de forma espontánea.

Con estas ideas en mente, es posible analizar los resultados obtenidos. En primer lugar, el agregado y calentamiento del precursor de $\mathrm{Si}\left(\mathrm{Mg}_{2} \mathrm{Si}\right.$, el cual es poco soluble en el medio de reacción) permite una mayor concentración de precursor soluble, lo cual facilita la reacción por vía homogénea. A su vez, el agregado de oxidante seguido de un aumento rápido en la temperatura favorecen la formación de núcleos de cristalización. 
Al variar la cantidad de oxidante, dejando la cantidad de precursor de Si fija, aumenta la cantidad de núcleos de crecimientos. Sin embargo, pueden obtenerse distintos escenarios:

- Manteniendo constante la sobresaturación de precursor de Si, se mantiene constante el número de núcleos y todos crecen de forma pareja, logrando una distribución estrecha.

- Si no se mantiene la sobresaturación, los núcleos inestables coalescen para formar cristales más grandes, y el ancho de la distribución de tamaños vendrá regulada por el tamaño de los núcleos coalescentes.

A partir de las distribuciones de tamaños experimentales, y como estas varían en función de la relación oxidante precursor de Si, podemos inferir que se trabajó en la segunda condición. En las condiciones de trabajo la cantidad de oxidante agregada regula la cantidad de núcleos de crecimiento presentes, y la cantidad de precursor de $\mathrm{Si}\left(\mathrm{Mg}_{2} \mathrm{Si}\right.$ en nuestro sistema) determina el crecimiento de los núcleos. Si la cantidad de precursor es insuficiente, los núcleos formados coalescen formando nanopartículas de mayor tamaño.

Para lograr un crecimiento más controlado, pueden emplearse distintas estrategias:

- Pueden hacerse agregados de precursor de Si a lo largo de la síntesis, para mantener la condición de sobresaturación de la solución y evitar la coalescencia hasta la formación de nanocristales estables.

- Calentar aún más el precursor de Si, y realizar un agregado en frío del oxidante, a fin de generar una nucleación brusca, y luego agregar más precursor en caliente y controlar el crecimiento de los núcleos.

- Una vez formados los núcleos, agregar un par reactivo alternativo (precursor de Si + co-reactivo, que puede ser tanto un oxidante o reductor según el precursor elegido) que presente mayor reactividad, con lo cual puede controlarse el crecimiento a menores temperaturas.

- Agregar agentes estabilizantes que se adsorban en la superficie y limiten la llegada de material a las caras cristalinas en crecimiento. Esta estrategia puede emplearse para la formación de nano-objetos anisotrópicos, si es que el estabilizante se adsorbe selectivamente sobre una cara cristalina.

A su vez, al estudiar las imágenes de HR-TEM obtenidas no pudo concluirse que las muestras sean cristalinas, con lo cual serán consideradas amorfas de aquí en adelante. Sin embargo, usando la misma vía sintética pero utilizando como precursor NaSi obtuvimos muestras claramente cristalinas. Atribuimos esta sutil diferencia a las distintas estructuras cristalinas del $\mathrm{NaSi}$ y $\mathrm{Mg}_{2} \mathrm{Si}$ : El primero cristaliza en una celda monoclínica, formando polianiones $\mathrm{Si}_{4}{ }_{4-}$ [5][6]; mientras que el segundo lo hace en una celda cúbica centrada en las caras sin las formación de $\mathrm{Si}_{4}{ }^{4-}$ [7]. Estos polianiones funcionan como núcleos de cremiento, induciendo estructura cristalina desde los primeros estadíos de la formación de las nanopartículas y llevando a la diferencia observada.

Según el modelo del confinamiento cuántico, la variación en el tamaño de las nanopartículas conduciría al cambio en sus propiedades electrónicas. Para estudiar esto hicimos estudios de voltamperometría cíclica (CV, del inglés Cyclic Voltammertry) y fotoquímicos.

Las medidas electroquímicas se realizaron depositando Si nps sobre vidrios de FTO. La limpieza de los sustratos se realizó en 3 pasos. Primero fueron sonicados por 30 min en acetona, luego sumergidos en una solución $2 \mathrm{M}$ de $\mathrm{KOH}$ en etOH en ebullición y finalmente sonicados una vez más en acetona por $30 \mathrm{~min}$.

Una vez limpios los sustratos de FTO, se procedió a depositar las Si nps usando el siguiente procedimiento:

- $\quad$ Sobre los sustratos limpios, se gotearon dispersiones acuosas de Si nps hasta observar una gota de aproximadamente $5 \mathrm{~mm}$ de diámetro. El solvente se evaporó usando una corriente de nitrógeno.

- $\quad$ Con mucho cuidado, los sustratos goteados se sumergieron en una solución de 1,10bis(trimetoxisilil)decano $3 \mathrm{mM}$ en ciclohexano durante $20 \mathrm{hs}$.

- $\quad$ Finalmente, los electrodos se enjuagaron con abundante etOH y posteriormente con agua bidestilada. 
Usamos 1,10-bis(trimetoxisilil)decano para mejorar la estabilidad del depósito, ya que forma un film altamente entrelazado sobre el FTO capaz de retener las Si nps. De esta forma se desarrollaron los estudios electroquímicos con mayor facilidad, ya que las medidas usando Si nps en el electrolito soporte requerían grandes cantidades de analito, las cuales no disponíamos. Este reactivo se usa extensivamente en la preparación de electrodos, debido a su alta estabilidasd y poca actividad electroquímica [8]. De todos modos, se corroboró que el uso de 1,10-bis(trimetoxisilil)decano no afecte las propiedades estudiadas, por lo que comparamos con los valores obtenidos con Si nps en la solución electrolítica para la muestra S1, obteniendo resultados muy similares.

Este procedimiento se realizó para cada Si np estudiada en este capítulo, y como control para las medidas electroquímicas se utilizó un vidrio de FTO tratado de forma idéntica, pero sin el depósito de nanopartículas. Las medidas se hicieron por triplicado.
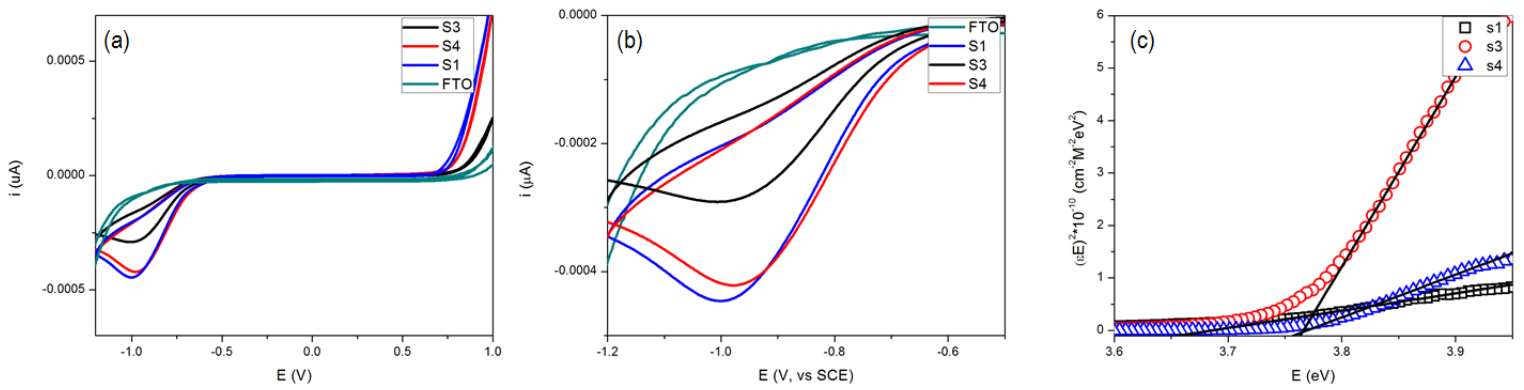

Figura 5.3. Medidas electroquímicas empleando las muestras sintetizadas. Los voltamperogamas (a) y (b) fueron realizados a 100 $\mathrm{mVs}^{-1}$ en una solución $0.1 \mathrm{M}$ de KOH , mientras que la estimación de la diferencia de energía entre las bandas de conducción y valencia $\left(E_{g}\right)$ se realizó a través de gráficos de Tauc $(c)$.

Las medidas de CV se realizaron en una celda de 3 electrodos (electrodo de trabajo: FTO con Si nps depositadas, referencia: calomel saturado SCE, contraelectrodo: hoja de Pt) barriendo a $100 \mathrm{mVs}^{-1}$ desde 1.00 $\mathrm{V}$ hasta $-1.20 \mathrm{~V}$, usando como electrolito soporte $\mathrm{KOH} 0.1 \mathrm{M}$ acuoso, a fin de obtener una ventana electroquímica lo más amplia posible.

Para estimar la diferencia de energía entre las bandas de conducción y valencia ( $E_{g}$, del inglés Energy Gap) usamos el espectro de atenuación óptico a través de gráficas de Tauc, estimando $E_{g}$ a partir de la extrapolación del comportamiento experimental en el borde de absorción [9]. El error asociado a la determinación está relacionado con el error en la extrapolación. El uso del espectro de atenuación para estimar el $E_{g}$ es necesario para deteminar la energía entre los estados vinculados al proceso de absorción. En caso de utilizar espectros de excitación de fotoluminiscencia, se determinaría la diferencia de energía entre el estado emisor y los estados que pueblan ese estado luego de la excitación (ya sea de forma directa o luego de una relajación vibracional). El comportamiento en el borde de absorción puede extrapolarse como:

$$
(a h v)^{r}=k(h v-E g)
$$

Donde a es el coeficiente de absorción del material estudiado (o una magnitud que sea lineal con a), hv es la energía de la radiación de excitación y Eg es la diferencia de energía entre la banda de valencia y la de conducción. Según sea el valor de $r$, la transición será directa y permitida $(r=2)$, directa y prohibida $(r=2 / 3)$, indirecta y permitida $(r=1 / 2)$ y indirecta y prohibida $(r=1 / 3)$.

A partir de la evidencia obtenida por medidas de conteo de fotones correlacionados en el tiempo (TCPSC, del inglés Time Correlated Single Photon Counting) y de lo discutido ampliamente en bibliografía [10]-[14], consideramos directa y permitida a la transición entre las bandas para la estimación. 
Las ondas voltamperométricas son claramente irreversibles, e incluso no llega a detectarse el proceso anódico en la ventana de potencial empleada (-1.2 V, $1 \mathrm{~V}$ vs SCE). Por lo tanto, los valores de potencial citados son los obtenidos para el pico catódico. Para estimar la posición del potencial para el proceso anódico se empleó $\mathrm{E}_{g}$, según la siguiente ecuación:

$$
e\left(E_{C B}-E_{V B}\right)=E_{g}
$$

Donde e es la carga del electrón, $E_{\mathrm{CB}}$ es el potencial de la banda de condución medido experimentalmente, y $E_{g}$ es el estimado a partir de gráficos de Tauc. Estos valores son estimaciones gruesas de los potenciales estándar para el proceso de reducción, ya que para poder determinar de forma precisa el valor de $\mathrm{E}^{0}$ es necesario tener información de ambos procesos, anódico y catódico.

\begin{tabular}{cccc}
\hline Muestra & $\mathbf{d}(\mathrm{nm})$ & $\mathrm{E}_{\mathrm{g}}(\mathrm{eV})$ & $\mathrm{E}_{\mathrm{CB}}(\mathrm{red}, \mathbf{V}$ vs NHE$)$ \\
\hline S1 & $3.4 \pm 0.6$ & $3.69 \pm 0.02$ & $-0.68 \pm 0.02$ \\
S3 & $4 \pm 1$ & $3.76 \pm 0.03$ & $-0.66 \pm 0.01$ \\
S4 & $5 \pm 1$ & $3.77 \pm 0.02$ & $-0.65 \pm 0.02$
\end{tabular}

Tabla 5.2. Propiedades electroquímicas medidas para distintas muestras analizadas en este capítulo.
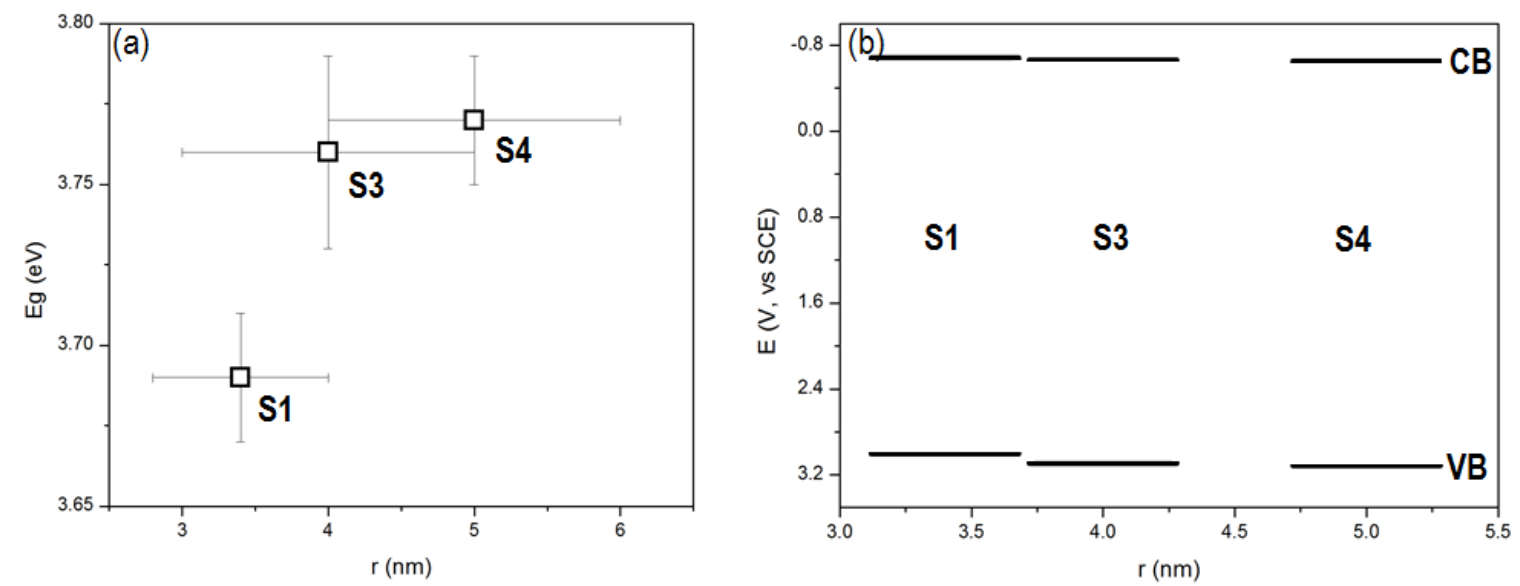

Figura 5.4. Variación de propiedades electroquímicas con el tamaño de las partículas.

Los resultados obtenidos se reflejan en las figuras 5.3 y 5.4, y en la tabla 5.1. Estos valores medidos de potencial se encuentran de acuerdo con lo estimado previamente por Llansola y colaboradores [15]. Los valores medidos de potencial disminuyen a medida que aumenta el diámetro de las nanopartículas, sin embargo el $\mathrm{E}_{\mathrm{g}}$ aumenta con este. Este comportamiento no es el esperado para un sistema que se comporte siguiendo un modelo de confinamiento cuántico, como se ha propuesto en bibliografía para Si nps cubiertas con diversos ligandos [16], [17]. En principio, es estrictamente válido esperar un comportamiento de tipo confinamiento cuántico si se dan una serie de condiciones:

- La nanoestructura considerada es lo suficientemente grande para que sus propiedades electrónicas puedan ser modeladas como un sólido cristalino extendido.

- Los ligandos no perturban la estructura electrónica de la nanoestructura, introduciendo estados en la región entre la banda de conducción y de valencia.

- Los estados superficiales o trampa no se localizan entre la banda de conducción y de valencia.

En caso de que alguno de estos requisitos no se cumpla, es posible que existan estados intermedios en la región energética entre la banda de conducción y de valencia, y que las transiciones ópticas involucren estos estados. De ser así, el $E_{g}$ estimado usando el espectro de atenuación óptica no es válido. En estos casos, el 
valor estimado representa la diferencia de energía entre los estados involucrados en la transición óptica que se manifiesta en el espectro de atenuación, y el $E_{g}$ debe estimarse si o si por vía electroquímica, haciendo una asignación cuidadosa de las ondas voltamperométricas. Como discutimos previamente, las Si nps obtenidas por esta vía son amorfas, con lo cual la aplicación del modelo de confinamiento cuántico no sería del todo correcto. Sin embargo, en Si nps amorfas existe una relación entre el borde de absorción y el tamaño aunque menos pronunciada que en caso de muestras cristalinas [18][19][20].

A partir de medidas de XPS y FTIR pudimos estimar la composición y la naturaleza de la superficie de las Si nps sintetizadas. Los resultados obtenidos son similares para las tres muestras estudiadas en este capítulo, pero por simpleza graficamos los resultados para Si nps de $3.4 \mathrm{~nm}$ de diámetro. En las figuras 5.5 y 5.6 mostramos como ejemplo espectros típicos FTIR y XPS de alta resolución para dicha muestra. A su vez, en la tabla 5.3 se listan las composiciones de todas las muestras de Si nps preparadas.

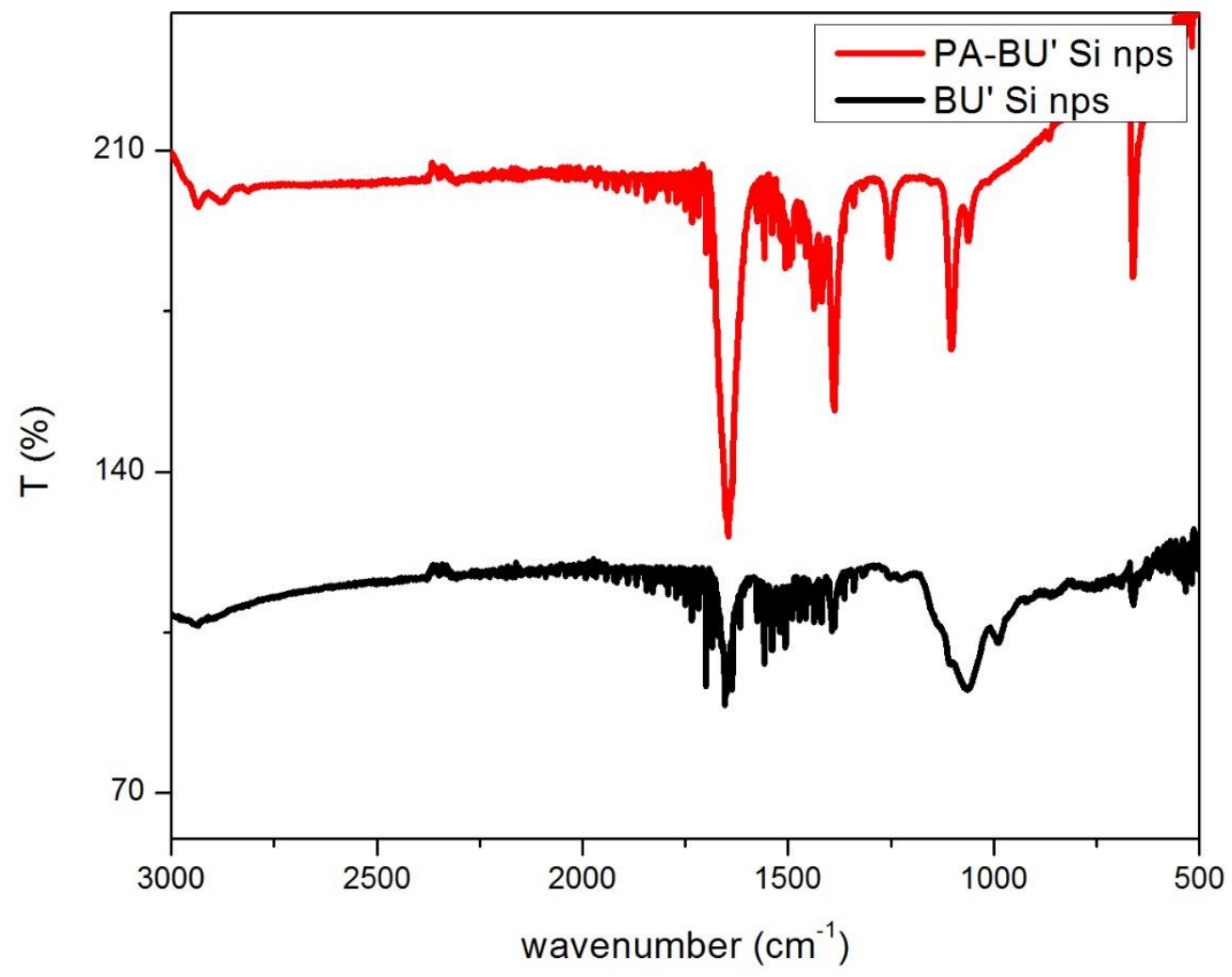

Figura 5.5. Espectros FTIR para PA-BU' Si nps (rojo) y BU' Si nps (negro) de $(3.4 \pm 0.6)$ nm de diámetro.

El espectro FTIR de las BU' Si nps (Figura 5.5, línea negra) muestra absorciones importantes en las regiones de 1040 y $1150 \mathrm{~cm}^{-1}$ asignables a vibraciones Si-O-Si [21], pero la ausencia de señales a $1250 \mathrm{~cm}^{-1}$ características del $\mathrm{SiO}_{2}$ masivo nos permiten descartar la formación de $\mathrm{SiO}_{2}$ en la superficie de las $\mathrm{Si}$ nps y considerar la formación de una capa oxidada sobre las nps, como fue reportado en casos estudiados en bibliografía [22]. Las señales a $1600 \mathrm{~cm}^{-1}$ pueden asignarse a solvente remanente (DMF).

En el caso de las PA-BU' Si nps, encontramos absorciones características de aminas primarias en las regiones $1100-1250$ y $1650 \mathrm{~cm}^{-1}$ debido a estiramientos y aleteos del enlace $\mathrm{C}-\mathrm{N}$, y en la zona de $660 \mathrm{~cm}^{-1}$, asignables

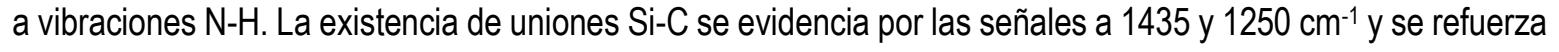


por las bandas a 1435 y $1270 \mathrm{~cm}^{-1}$ debido a deformaciones simétricas y asimétricas de metilenos en $\mathrm{Si}-\mathrm{CH}_{2}-$ [23]-[25].

A partir de las medidas de XPS pudimos realizar 2 análisis complementarios. Por un lado, usamos las relaciones entre las señales de $\mathrm{Si}, \mathrm{O}, \mathrm{C}, \mathrm{N}$ y $\mathrm{Cl}$ en el espectro XPS de barrido corregidas por los factores de sensibilidad instrumental que nos permitieron obtener la composición promedio de las distintas Si nps preparadas, como se ve en la tabla 5.3. Pel otro, se tomaron espectros de alta resolución en las regiones de $\mathrm{Si} 2 \mathrm{~s}, \mathrm{C}$ 1s y N $1 \mathrm{~s}$ para obtener mayor información sobre los entornos químicos presentes en las Si nps.

Es importante notar que la señal de $\mathrm{Cl}$ puede provenir de 2 fuentes: Primero, uno de los rectivos de síntesis es el $\mathrm{NH}_{4} \mathrm{Cl}$, con lo cual es posible una contaminación remanente en la solución depositada para el estudio; 0 también es posible que queden átomos de $\mathrm{Cl}$ unidos a la superficie, ya sea de forma covalente o adsorbidos. $\mathrm{A}$ su vez, la señal de $C$ no puede usarse de forma cuantitativa directamente, debido a la presencia de $C$ espurio normalmente en medidas de XPS. Para obtener los valores sugeridos en la tabla 5.3 se utilizó la corrección sugerida por Payne y colaboradores [26].

\begin{tabular}{ccc}
\hline Muestra & $\mathbf{d}(\mathbf{n m})$ & Composición \\
\hline S1 & $3.4 \pm 0.6$ & $\mathrm{Si}\left(\mathrm{O}_{0.46} \mathrm{C}_{1.88} \mathrm{~N}_{0.86} \mathrm{H}_{\mathrm{y}} \mathrm{Cl}_{0.05}\right)$ \\
S3 & $4 \pm 1$ & $\mathrm{Si}\left(\mathrm{O}_{0.37} \mathrm{C}_{1.90} \mathrm{~N}_{0.78} \mathrm{H}_{\mathrm{y}} \mathrm{Cl}_{0.03}\right)$ \\
S4 & $5 \pm 1$ & $\mathrm{Si}\left(\mathrm{O}_{0.39} \mathrm{C}_{1.76} \mathrm{~N}_{0.80} \mathrm{H}_{\mathrm{y}} \mathrm{Cl}_{0.07}\right)$
\end{tabular}

Tabla 5.3. Composición de las muestras de PA'BU' Si nps preparadas.

A partir del análisis cuantitativo de los datos de XPS, vimos que la composición no varió fuertemente (dentro del error experimental de la técnica, que es de $\pm 10 \%$ ) con el tamaño. Si tenemos en cuenta que el área superficial $x$ nanopartícula aumenta con el diámetro, el grado de cubrimiento por PA disminuye a medida que aumenta el tamaño de las partículas en las condiciones de síntesis utilizadas.
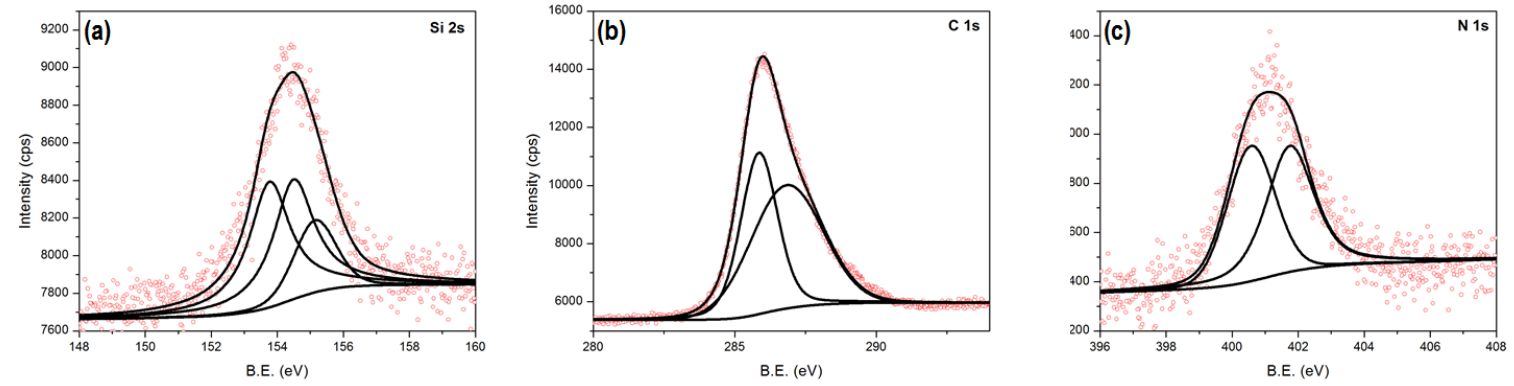

Figura 5.6. Espectros XPS de alta resolución para PA-BU' Si nps de $(3.4 \pm 0.6) \mathrm{nm}$ de diámetro, en las regiones (a) Si $2 \mathrm{~s}$, (b) C 1s y (c) N1s. Los círculos rojos muestran los datos experimentales, mientras que la línea negra las componentes ajustadas.

El estudio de los espectros XPS de alta resolución (figura 5.6) re-afirmó las asignaciones del especto FTIR. En la región de $\mathrm{C} 1 \mathrm{~s}$, los picos se deconvolucionaron en 2 contribuciones centradas en $284.5-284.8 \mathrm{eV}$, asignable a C alquílicos, y en 285.6 - 285.9 eV correspondientes a C adyacentes a grupos aminos [27][28][29]. A su vez, la señal de la región de $\mathrm{N}$ 1s puede asignase como 2 señales solapadas, una centrada en 402.3 - $402.8 \mathrm{eV}$ que asignamos a aminas protonadas [11], [30], [31], y otra en $400.5-400.6 \mathrm{eV}$ asignada a entornos del $\mathrm{N}$ conteniendo C [27], [32][29].Finalmente, la señal detectada en la región de Si 2s pudo analizarse como 3 contribuciones superpuestas: La primera centrada en 152.4 - $152.6 \mathrm{eV}$, reportada en bibliografía para sistemas SiOx/Si y en subóxidos [27], [32], [33], una segunda en 153.3 - 153.4 eV asignada a Si en entornos polares en presencia de N y C [29], [34], [35], y finalmente en 153.9 - $154.15 \mathrm{eV}$, reportada para Si en entornos alquílicos [36]. Mostramos los espectros obtenidos en la figura 5.6. 
A partir de la información otenida, tuvimos éxito en la síntesis de Si nps por oxidación de $\mathrm{Mg}_{2} \mathrm{Si}$ (llamadas BU' Si nps a lo largo de este trabajo) y en la regulación de su tamaño cambiando las condiciones experimentales de síntesis. Las nps obtenidas no mostraron claros indicios de cristalinidad, lo cual atribuimos a la estructura cristalina del precursor, ya que al cambiarlo por $\mathrm{NaSi}$ con propiedades cristalinas diferentes, se obtuvieron $\mathrm{Si}$ nps cristalinas. Finalmente, luego de cubrir las Si nps con 2-propenil-amina a través de una reacción de sililación por vía térmica, se corroboró la presencia de grupos alquilamina y de cierto grado de oxidación en la superficie de las Si nps a través de medidas de FTIR y XPS.

\subsubsection{Experimentos fotofísicos}

Realizamos una serie de estudios fotofísicos con las PA-BU' Si nps de distintos tamaños sintetizadas, a fin de regular a través del tamaño la naturaleza de los estados vinculados a la absorción y como estos interactúan con los estados responsables de la emisión. Utilizamos experimentos de absorción y emisión resueltos en el tiempo y empleando luz polarizada.
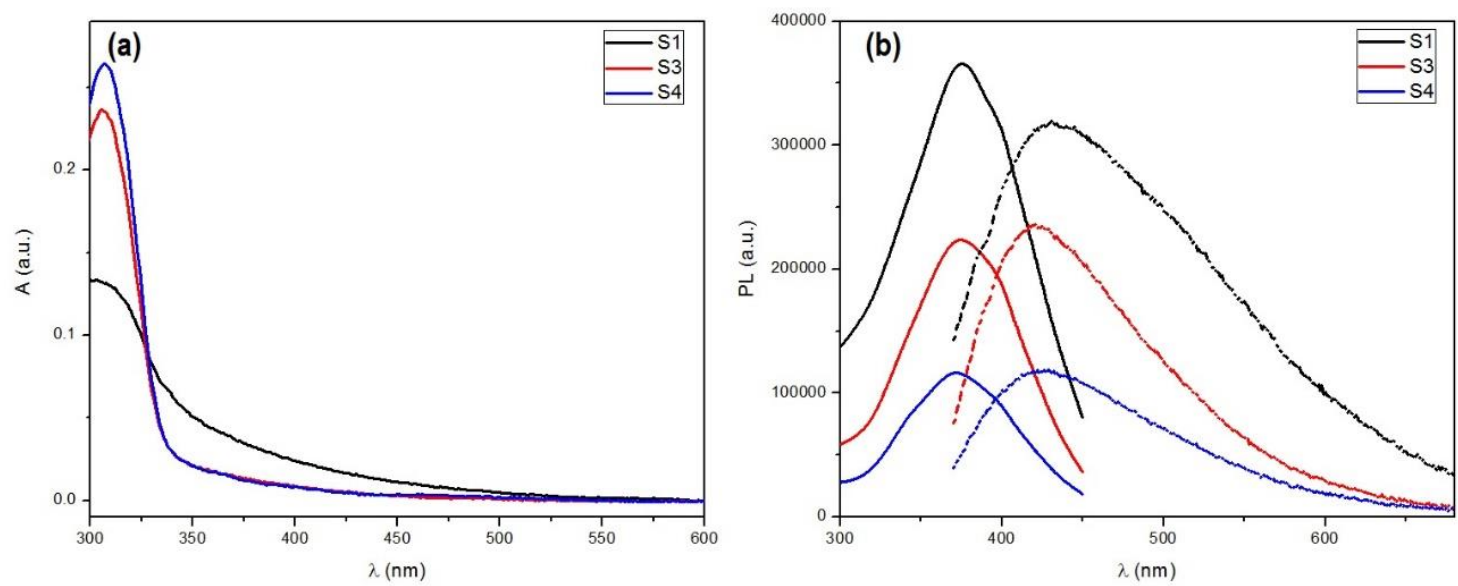

Figura 5.7. Espectros de (a) atenuación, (b) emisión $\left(\lambda_{\mathrm{ex}}=350 \mathrm{~nm}\right.$, línea punteada) y excitación ( $\lambda_{\mathrm{em}}=470 \mathrm{~nm}$, línea continua) para las BU' Si nps de distinto tamaño. Los espectros que presentamos están tomados en dispersiones $0.2 \mu \mathrm{M}$ en DMF.

Los espectros de atenuación, emisión (excitando a $350 \mathrm{~nm}$ ) y excitación (detectando a $470 \mathrm{~nm}$ ) estacionarios medidos (figura 5.7) están de acuerdo con lo reportado en bibliografía: Espectros de atenuación estrechos, excitación estrecha en el violeta y espectros de emisión anchos y que se distribuyen a lo largo de la región azulverde del espectro electromagnético [12], [15], [23], [29]. Usando los especros de excitación, estimamos las diferencias de energía entre el estado emisor y los estados que pueblan ese estado luego de la excitación (nombrado como $\mathrm{E}_{g}^{\prime}$ ) para las distintas muestras preparadas. Mostramos estos valores en la tabla 5.4.

A su vez, cuanto más ancha sea la distribución de tamaños más ancha será la distribución de espectro de emisión. Empleando la misma configuración experimental se tomaron los mismos espectros para 9,10-DPA, y calculamos los rendimientos cuánticos de emisión para las muestras sintetizadas. En el rango experimental estudiado, encontramos que al aumentar el tamaño de las Si nps aumenta $\Phi_{\text {em }}$. Los rendimientos cuánticos medidos se muestran en la tabla 5.4 .

Hicimos distintos experimentos de emisión resuelta en el tiempo bajo la metodología de conteo de fotones correlacionados en el tiempo (TCPSC) excitando a $341 \mathrm{~nm}$ y $388 \mathrm{~nm}$. De estos experimentos se desprendió el comportamiento multiexponencial del decaimiento del estado excitado, obteniendo, dentro del error experimental, constantes de tiempo iguales para ambas condiciones de excitación. A partir de las medidas de tiempos de vida en el máximo de emisión obtuvimos los tiempos de vida media $\langle T\rangle$, mientras que usando TRES estudiamos la evolución del espectro de emisión a lo largo del tiempo de vida del estado excitado. Para analizar 
las medidas de TRES usamos análisis global y obtuvimos un comportamiento bi-exponencial en todo el intervalo espectral estudiado. Los espectros asociados a cada decaimiento (DAS, del inglés Decay Associated Spectra) se muestran en la figura 5.8. Observamos que la variación en el tamaño de las Si nps conduce a cambios tanto en $\langle\mathrm{T}>$ como en la distribución de los DAS.
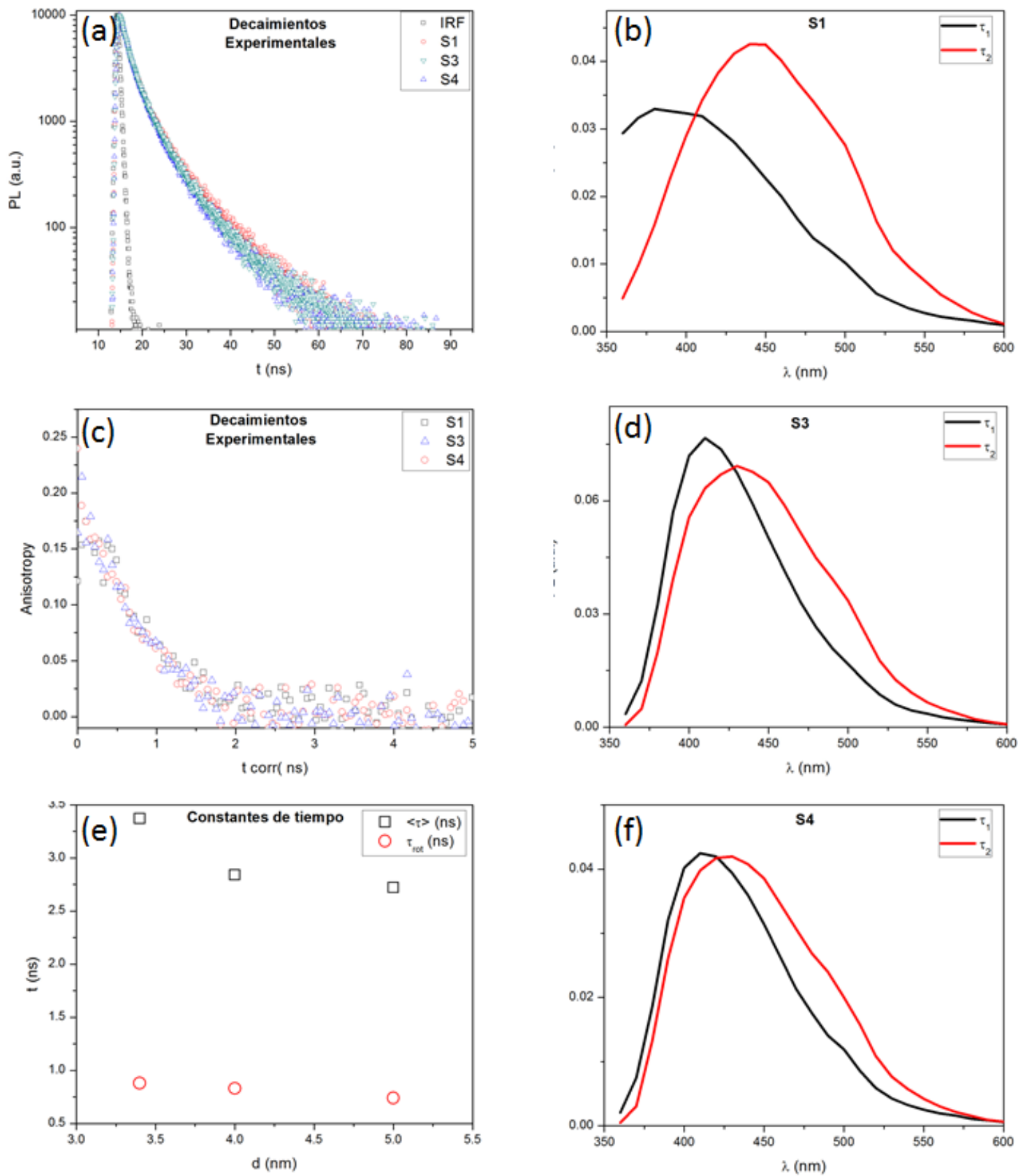

Figura 5.8. Experimentos de emisión resuelta en el tiempo $\left(\lambda_{\mathrm{ex}}=341 \mathrm{~nm}\right)$. (a) Decaimientos de la intensidad y (c) anisotropía de emisión para las distintas muestras preparadas. Presentamos también los DAS para muestras (b) S1, (d) S3 y (f) S4. Las variaciones de las constantes de tiempo en función del tamaño de las nps se muestran en (e), siendo los círculos rojos los tiempos de correlación rotacional y los cuadrados negros son los tiempos de vida media.

Realizamos experimentos de anisotropía de emisión para obtener mayor información del proceso de relajación del estado excitado y tamaño de las partículas PL encontrando que tanto los tiempos de despolarización 
rotacional ( $\left.\mathrm{Trot}_{\mathrm{rot}}\right)$ como la anisotropía a tiempo cero $\left(\mathrm{r}_{0}\right)$ no varían sustancialmente en las muestras estudiadas. El hecho de que $r_{0}$ no varíe con el tamaño de las nps indica que el estado desde el cual se produce la relajación por vía radiativa NO está influenciado por este [14]. A su vez, que $r_{0}$ sea distinto de 0.4 indica que los vectores momento de transición ( $\left.\boldsymbol{\mu}_{\mathrm{if}}\right)$ para los procesos de absorción y emisión no son paralelos, con lo cual los estados involucrados en estos procesos son distintos.

Los parámetros que caracterizan al proceso de emisión para cada muestra se listan a continuación en la tabla 5.4 .

\begin{tabular}{cccc}
\hline Propiedad & \multicolumn{3}{c}{ Muestras } \\
& S1 & S3 & S4 \\
\hline$d(n m)$ & $3.4 \pm 0.6$ & $4 \pm 1$ & $5 \pm 1$ \\
$\lambda_{\text {abs, } \max (n m)}$ & $305.0 \pm 0.5$ & $311.5 \pm 0.5$ & $311.0 \pm 0.5$ \\
$\lambda_{\text {em, } \max (n m)}$ & $432.0 \pm 0.5$ & $421.0 \pm 0.5$ & $428.0 \pm 0.5$ \\
$\lambda_{\text {ex, } \max }$ & $375.0 \pm 0.5$ & $373.0 \pm 0.5$ & $370.0 \pm 0.5$ \\
$E_{g}(e V)$ & $3.69 \pm 0.02$ & $3.76 \pm 0.03$ & $3.77 \pm 0.02$ \\
$E_{g}(\mathrm{eV})$ & $2.64 \pm 0.03$ & $2.68 \pm 0.02$ & $2.67 \pm 0.02$ \\
$\Phi_{\mathrm{em}}\left(\lambda_{\mathrm{ex}}=350 \mathrm{~nm}\right)$ & $0.11 \pm 0.03$ & $0.39 \pm 0.05$ & $0.64 \pm 0.02$ \\
$\mathrm{~T}_{1}(\%)(\mathrm{ns})$ & $1.40(64.0)$ & $1.75(80.0)$ & $1.56(78.0)$ \\
$\mathrm{T}_{2}(\%)(\mathrm{ns})$ & $6.88(36.0)$ & $7.20(20.0)$ & $6.80(22.0)$ \\
$<\mathrm{T}>(\mathrm{ns})$ & $3.37 \pm 0.03$ & $2.84 \pm 0.05$ & $2.72 \pm 0.03$ \\
$\mathrm{t}_{\mathrm{rot}}(\mathrm{ns})$ & $0.88 \pm 0.08$ & $0.83 \pm 0.05$ & $0.74 \pm 0.04$ \\
$\mathrm{r}_{0}$ & $0.210 \pm 0.006$ & $0.223 \pm 0.006$ & $0.225 \pm 0.007$
\end{tabular}

Tabla 5.4. Resumen de propiedades fotofísicas determinadas usando medidas de emisión.

Existen en literatura distintos aportes sobre Si nps que emiten en la banda S [24], [37]-[39], pero el origen y comportamiento de la banda $F$ aun está en discusión [12], [23], [40], [18]. Actualmente se está probando la hipótesis de que la emisión en la banda $\mathrm{F}$ está relacionada con estados trampa debidos a defectos relacionados a $\mathrm{N}$ y $\mathrm{O}$, sin embargo la relación de estos estados con los estados finales del proceso de absorción y sus acoplmamientos aún no están claros.

Teniendo en cuenta que la composición y los grupos superficiales son similares para todas las muestras preparadas, las diferencias encontradas en el comportamiento fotofísico se deben a las variaciones en los canales que vinculan los estados involucrados en los procesos de absorción y emisión. A su vez, es necesario tener presente que las Si nps estudiadas no son cristalinas, con lo cual su fotofísica no puede estudiarse considerando solamente un modelo de confinamiento cuántico.

Para obtener mayor información realizamos experimentos de emisión resueltos en el tiempo. El comportamiento multiexponencial observado puede explicarse considerando diferentes estados excitados involucrados en la emisión y excitación. La existencia de múltiples niveles vinculados que afectan la fotofísica de un sistema puede explicar el comportamiento observado suponiendo un modelo simple de 2 niveles interactuantes. En este caso, el cambio en los DAS puede explicarse con cambios en la diferencia energética entre los niveles involucrados.

Por otro lado, considerar un solo tiempo de despolarización implica para el modelo de niveles vinculados que la despolarización entre estos (y por lo tanto su interconversión) se da por debajo de la escala temporal estudiada. Entonces, las constantes de velocidad de estos procesos están por encima de $0.005 \mathrm{ps}^{-1}$ (inversa del error en la determinación de t en los experimentos de TCPSC en la configuración experimental disponible en INIFTA). 
Para obtener información sobre los procesos en la escala de los sub-nanosegundos utilizamos la técnica de espectroscopía de absorción de transientes en los femto y picosegundos (TAS, del inglés Trasient Absorption Spectroscopy). Realizamos todas las medidas en las mismas condiciones experimentales ( $\lambda_{\mathrm{ex}}=400 \mathrm{~nm}, 130$ $\mu \mathrm{j} /$ pulso, $\mathrm{A}_{400} \sim 1 \mathrm{y}$ usando un supercontinuo de luz de análisis lo más estable posible a lo largo de los experimentos), para poder establecer diferencias que respondan puramente al cambio en la estructura electrónica de las distintas Si nps. Mostramos algunas trazas obtenidas en la figura 5.9.
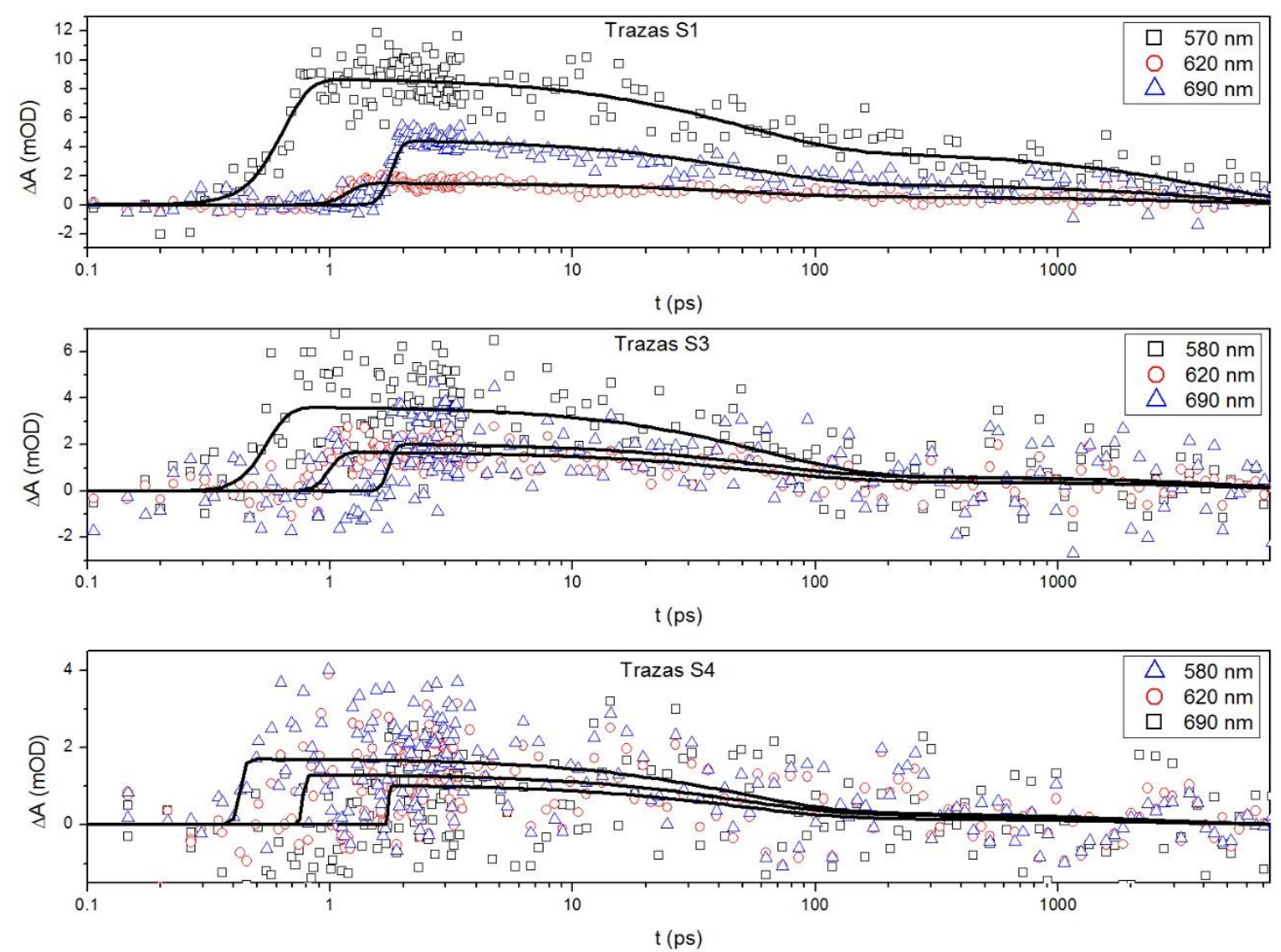

Figura 5.10. Trazas obtenidas en experimentos de TAS en las Si nps sintetizadas en este capítulo. Las condiciones experimentales fueron $\lambda_{\mathrm{ex}}=400 \mathrm{~nm}, 130 \mathrm{\mu j} /$ pulso y $\mathrm{A}_{400} \sim 1$.

Para analizar los datos obtenidos, utilizamos un modelo de 3 niveles como el que se propone en la siguiente figura. 


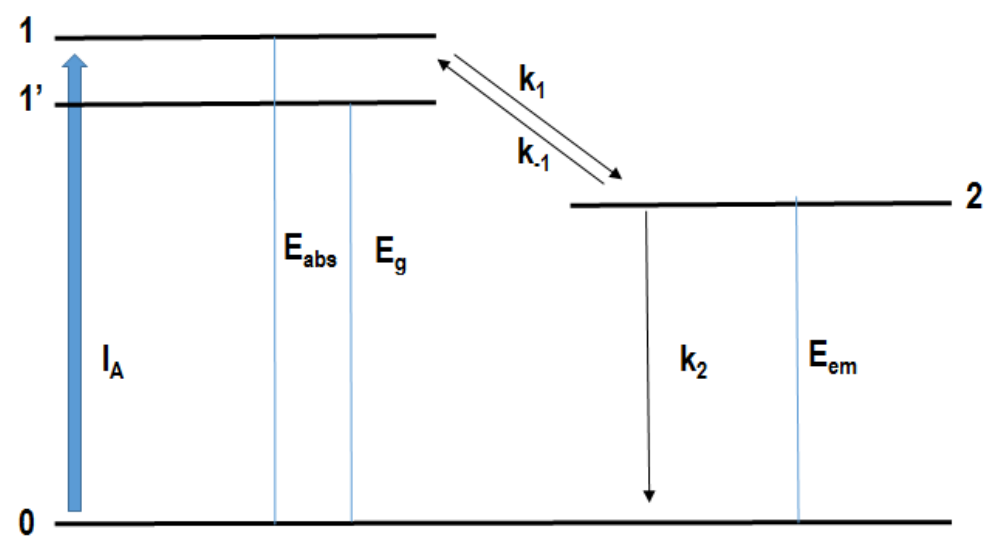

Figura 5.10. Modelo de 3 niveles propuesto para explicar la fotofísica de las PA-BU' Si nps.

En este esquema, la absorción se da entre el nivel fundamental 0 y un nivel 1 excitado. Este nivel 1 tiene energía $E_{a b s}$ y puede o no coincidir con aquel cuya energía coincida con Eg (1'). El nivel 1 es poblado directamente en el proceso de absorción con un pulso $I_{A}$, y luego decae al nivel 2 con una constante de velocidad $k_{1}$. Luego 2 evoluciona retornando a 1 vía $k_{-1}$ o relajando al nivel fundamental 0 vía $k_{2}$. Suponemos que 2 es el nivel emisor, con lo cual la diferencia de energía entre 2 y 0 es $E_{e m}$. Los valores de $E_{a b s,} E_{e m}$ y $E_{g}$ fueron estimados a partir de los máximos de absorción, emisión y del umbral de absorción, respectivamente, para cada muestra. Es importante tener en cuenta que estas estimaciones son groseras, y se utilizan solamente para evaluar la factibilidad de los procesos propuestos.

Usando este modelo se obtuvo un buen ajuste a los datos experimentales, obteniendo los espectros asociados a cada especie (SAS, del inglés Species Associated Spectra) que se muestran en la figura 5.11. Los espectros en línea negra corresponden a 1, mientras que el espectro en línea roja a 2. En ambos casos vemos absorciones fotoinducidas, sin emisión estimulada ni blanqueos del estado fundamental (al menos no en la región sondeada). Podemos ver que al aumentar el tamaño de las $\mathrm{np}$, el espectro de absorción de 1 se ensancha si bien el máximo se presenta en la misma posición. A su vez, el espectro obtenido para 2 presenta picos más angostos en el lado azul del espectro. Los SAS obtenidos están de acuerdo con los reportados previamente por Kryschi, Venoit y Kauzlarich [40]-[42]. El hecho de que los espectros obtenidos para 1 en los 3 casos muestre la misma posición pero distintos anchos sugiere que la naturaleza de este estado es similar para todas las muestras pero la densidad de estados que puedan interactuar con 1 cambia. Si tenemos en cuenta la propuesta para Si nps que emiten en la banda $\mathrm{F}$ el estado emisor (2) es un estado trampa asociados a $\mathrm{N}$ u O, el cambio en el cubrimiento por $\mathrm{N}$ y $\mathrm{O}$ observado para estas nps puede explicar los cambios espectrales:

- Al haber menor densidad de estados 2 que atrapen a 1, el espectro observado para 1 se ensancha al disminuir el cubrimiento $\mathrm{x} \mathrm{N}$ y $\mathrm{O}$.

- Al haber menor cubrimiento de $\mathrm{N}$ y $\mathrm{O}$, el espectro de 2 se resuelve de forma más pobre y no se evidencia claramente su forma (como sucede en S4).

Es importante considerar que a partir de la evidencia experimental no puede descartarse que estos cambios en el comportamiento del estado excitado estén vinculados a la agregación del sistema. Sin embargo, los efectos de la aglomeración sobre la fotofísica de Si nps se manifiestan, según lo hallado en literatura, en la escala desde cientos de ns a los $\mu$ s [43], [44][45] 

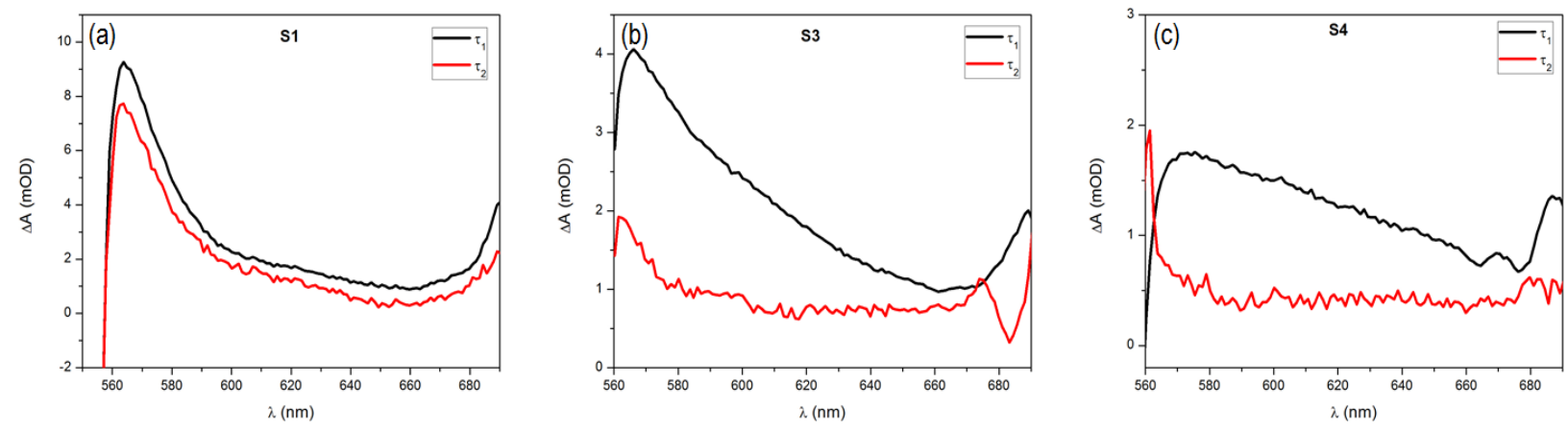

Figura 5.11. SAS obtenidos luego de analizar los experimentos de TAS para (a) S1, (b) S3 y (c) S4.

A su vez, las constantes de velocidad propias del modelo cambian con la muestra estudiada, lo cual refleja el cambio en la estructura electrónica. Los parámetros que calculamos se muestran en la tabla 5.5. Es importante notar que la inversa de la suma de las constantes involucradas en la evolución de 2 representan el tiempo de vida medio de dicho estado, el cual coincide con el determinado en experimentos de TCSPC, tanto excitando a $341 \mathrm{~nm}$ como $388 \mathrm{~nm}$. Se observa que, si bien $\mathrm{k}_{1}$ no presenta variaciones importantes para las distintas muestras, si lo hace $\mathrm{k}_{-1}$. Esta constante está vinculada con la probabilidad de poblar 1 desde 2, con lo cual su disminución al aumentar la diferencia de energía entre los estados 1 y $2\left(\Delta \mathrm{E}_{1}\right)$ es lógica. De hecho, estas variables guardan una buena correlación experimental $\left(r^{2}=0.9978\right)$ como muestra la figura 5.12. Para verificar que los estados vinculados eran 1 y 2 , sin la necesidad de pasar por los estados en el fondo de la banda de conducción (1'), se evaluó la posible dependencia de k-1 con la diferencia de energía entre 1' y $2\left(\Delta \mathrm{E}_{2}\right)$, sin encontrarse una correlación como en el caso previo.

\begin{tabular}{ccccccc}
\hline Muestra & $\mathrm{k}_{1}\left(\mathrm{ps}^{-1}\right)$ & $\mathrm{k}_{-1}\left(\mathrm{ps}^{-1}\right)$ & $\mathrm{k}_{2}\left(\mathrm{ps}^{-1}\right)$ & $\left(\mathrm{k}_{-1}+\mathrm{k}_{2}\right)^{-1}(\mathrm{ps})$ & $\Delta \mathrm{E}_{1}(\mathrm{ae}, \mathrm{eV})$ & $\Delta \mathrm{E}_{2}(\mathrm{ge}, \mathrm{eV})$ \\
\hline S1 & 0.01 & 0.0001 & 0.0002 & 3333.3 & 1.81 & 1.44 \\
S3 & 0.008 & $2.50 \mathrm{E}-07$ & 0.00035 & 2855.1 & 2.88 & 1.9 \\
S4 & 0.01 & $8.00 \mathrm{E}-06$ & 0.00036 & 2717.4 & 2.76 & 1.21
\end{tabular}

Tabla 5.5. Parámetros cinéticos obtenidos usando el modelo propuesto. Se listan también las diferencias energéticas entre los estados 1 y $2\left(\Delta \mathrm{E}_{1}\right)$ y el fondo de la banda de conducción y $2\left(\Delta \mathrm{E}_{2}\right)$. 


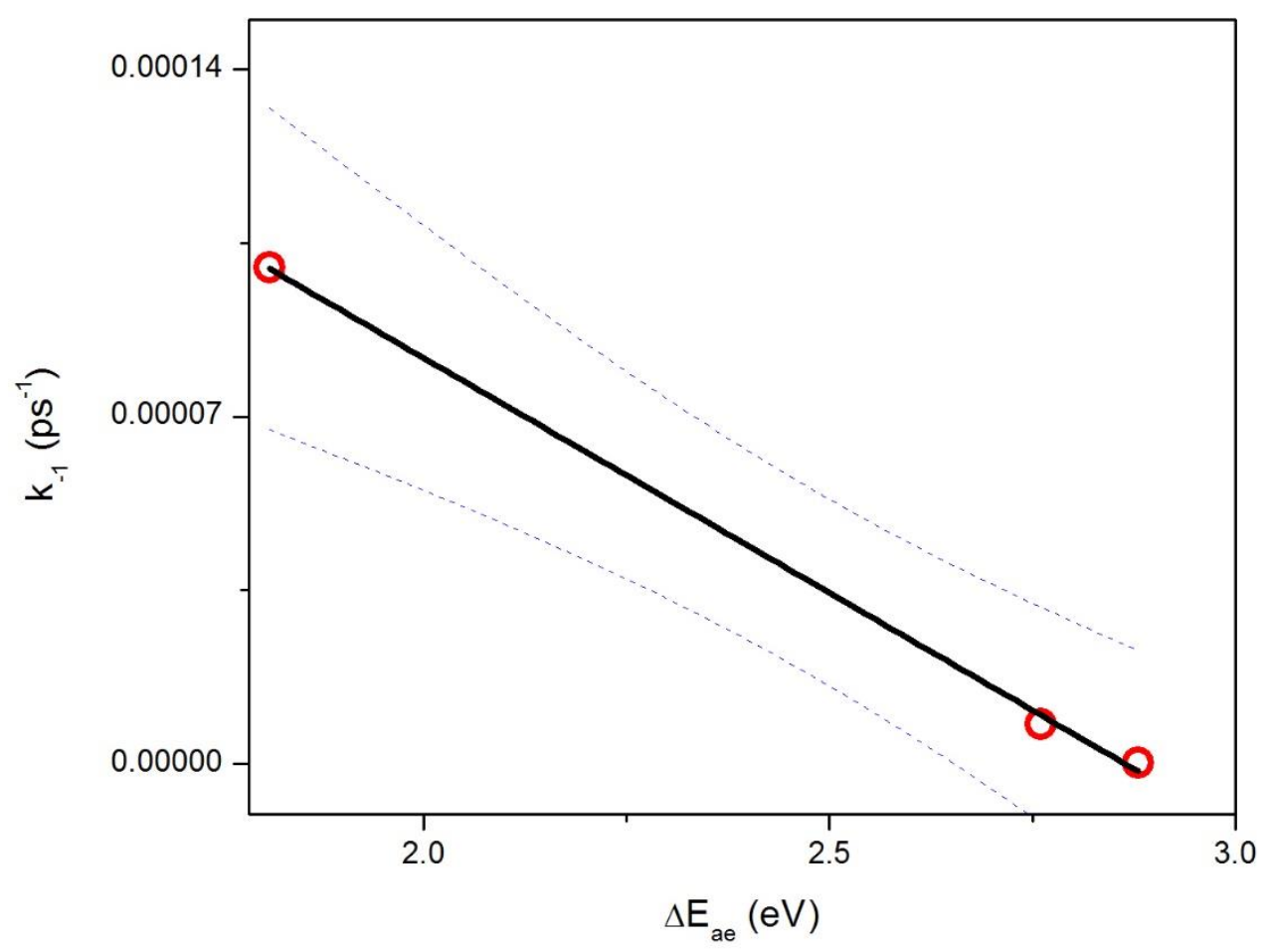

Figura 5.12. Variación de k-1 en función de la diferencia de energía entre los niveles 1 y 2.

A partir de la evidencia experimental adquirida, proponemos que el modelo de 3 niveles empleado explica satisfactoriamente el comportamiento fotofísico de las PA-BU' Si nps estudiadas en este capítulo, permitiendo la explicación del comportamiento multi-exponencial observado en experimentos de TCSPC y TAS.

\subsection{Conclusiones}

Se sintetizaron exitosamente Si nps por oxidación de fases de Zintl en fase orgánica, posteriormente fueron cubiertas con 2-propenilamina. Estas PA-BU' Si nps fueron caracterizadas empleando HR-TEM, ftir y xps. Variando las condiciones de síntesis se logró controlar el tamaño de las Si nps obtenidas, lo cual permitió sintonizar ciertas propiedades ópticas y electroquímicas. A tavés de experimentos ópticos de TCPSC y TAS, pudimos establecer que si bien los estados involucrados en el proceso de absorción pueden controlarse ajustando las condiciones sintéticas, los estados emisores están asociados a trampas de $\mathrm{O}$ o N, cuya emisión se ve modificada levemente según el tamaño de las Si nps, sino más bien por la distribución de trampas superficiales relacionadas al cubrimiento con $\mathrm{N}$ y $\mathrm{O}$. A su vez, es importante recordar que a partir de la evidencia experimental no puede concluirse nada sobre el efecto de la aglomeración de las Si nps sobre su fotofísica.

El método sintético desarrollado en este capítulo fue utilizado en el resto de los experimentos involucrados a este trabajo de tesis, debido a su alta reproducibilidad y fundamentalmente por la simpleza con la cual pueden obtenerse cantidades en el orden de los mg de partículas, lo cual es fundamental para experimentos ópticos de absorción de transientes en los fs. 


\subsection{Referencias}

[1] D. Neiner, H. W. Chiu, and S. M. Kauzlarich, "Low-temperature solution route to macroscopic amounts of hydrogen terminated silicon nanoparticles," J. Am. Chem. Soc., vol. 128, pp. 11016-11017, 2006.

[2] R. K. Baldwin, K. a. Pettigrew, J. C. Garno, P. P. Power, G. Y. Liu, and S. M. Kauzlarich, "Room temperature solution synthesis of alkyl-capped tetrahedral shaped silicon nanocrystals," J. Am. Chem. Soc., vol. 124, pp. 1150-1151, 2002.

[3] K. Pettigrew and Q. Liu, "Solution synthesis of alkyl-and alkyl/alkoxy-capped silicon nanoparticles via oxidation of Mg2Si," Chem. ..., no. 31, pp. 4005-4011, 2003.

[4] V. LaMer and R. Dinegar, "Theory, production and mechanism of formation of monodispersed hydrosols," J. Am. Chem. ..., vol. 72, no. 8, pp. 4847-4854, 1950.

[5] T. Goebel, Y. Prots, and F. Haarmann, "Refinement of the crystal structure of tetrasodium tetrasilicide , Na 4 Si 4," vol. 223, pp. 187-188, 2008.

[6] S. Yamanaka, "Silicon clathrates and carbon analogs: high pressure synthesis, structure, and superconductivity.," Dalton Trans., vol. 39, pp. 1901-1915, 2010.

[7] J. M. Chem, T. Yi, S. Chen, S. Li, H. Yang, S. Bux, Z. Bian, N. A. Katcho, A. Shakouri, N. Mingo, J. Fleurial, D. Browning, and S. M. Kauzlarich, "Synthesis and characterization of Mg $2 \mathrm{Si} / \mathrm{Si}$ nanocomposites prepared from MgH 2 and silicon , and their thermoelectric properties †," pp. 2480524813, 2012.

[8] J. D. Megiatto, A. Antoniuk-Pablant, B. D. Sherman, G. Kodis, M. Gervaldo, T. A. Moore, A. L. Moore, and D. Gust, "Mimicking the electron transfer chain in photosystem II with a molecular triad thermodynamically capable of water oxidation," Proc. Natl. Acad. Sci., vol. 109, no. 39, pp. 1557815583, 2012.

[9] J. Tauc, "Optical properties and electronic structure of amorphous Ge and Si," Mater. Res. Bull., vol. 3, no. 1, pp. 37-46, 1968.

[10] J. G. C. Veinot, "Synthesis, surface functionalization, and properties of freestanding silicon nanocrystals.," Chem. Commun. (Camb)., pp. 4160-4168, 2006.

[11] J. J. Romero, M. J. Llansola-Portolés, M. L. Dell'Arciprete, H. B. Rodríguez, A. L. Moore, and M. C. Gonzalez, "Photoluminescent 1-2 nm sized silicon nanoparticles: A surface-dependent system," Chem. Mater., vol. 25, pp. 3488-3498, 2013.

[12] S. Yang, W. Li, B. Cao, H. Zeng, and W. Cai, "Origin of blue emission from silicon nanoparticles: Direct transition and interface recombination," J. Phys. Chem. C, vol. 115, pp. 21056-21062, 2011.

[13] M. J. Llansola Portolés, F. R. Nieto, D. B. Soria, J. I. Amalvy, P. J. Peruzzo, D. O. Mártire, M. Kotler, O. Holub, and M. C. Gonzalez, "Photophysical properties of blue-emitting silicon nanoparticles," J. Phys. Chem. C, vol. 113, pp. 13694-13702, 2009.

[14] M. Dasog, Z. Yang, S. Regli, T. M. Atkins, A. Faramus, M. P. Singh, E. Muthuswamy, S. M. Kauzlarich, R. D. Tilley, and J. G. C. Veinot, "Chemical insight into the origin of red and blue photoluminescence arising from freestanding silicon nanocrystals," ACS Nano, vol. 7, no. 3, pp. 2676 2685, 2013. 
[15] M. J. Llansola Portolés, P. M. David Gara, M. L. Kotler, S. Bertolotti, E. San Román, H. B. Rodríguez, and M. C. Gonzalez, "Silicon nanoparticle photophysics and singlet oxygen generation," Langmuir, vol. 26, no. 13, pp. 10953-10960, 2010.

[16] Z. F. Li, M. T. Swihart, and E. Ruckenstein, "Luminescent silicon nanoparticles capped by conductive polyaniline through the self-assembly method," Langmuir, vol. 20, no. 19, pp. 1963-1971, 2004.

[17] F. Hua, M. T. Swihart, and E. Ruckenstein, "Efficient surface grafting of luminescent silicon quantum dots by photoinitiated hydrosilylation," Langmuir, vol. 21, no. 18, pp. 6054-6062, 2005.

[18] O. Lehtonen and D. Sundholm, "Bright luminescence from silane substituted and bridged silicon nanoclusters.," Phys. Chem. Chem. Phys., vol. 8, pp. 4228-4232, 2006.

[19] R. Wang, X. Pi, and D. Yang, "First-Principles Study on the Surface Chemistry of $1.4 \mathrm{~nm}$ Silicon Nanocrystals : Case of Hydrosilylation," 2012.

[20] G. Ledoux, O. Guillois, D. Porterat, C. Reynaud, F. Huisken, B. Kohn, and V. Paillard, "Photoluminescence properties of silicon nanocrystals as a function of their size," Phys. Rev. B, vol. 62, no. 23, pp. 15942-15951, 2000.

[21] A. Shiohara, S. Hanada, S. Prabakar, K. Fujioka, T. H. Lim, K. Yamamoto, P. T. Northcote, and R. D. Tilley, "Chemical Reactions on Surface Molecules Attached to Silicon Quantum Dots," J. Am. Chem. Soc., vol. 132, no. 24, pp. 248-253, 2009.

[22] M. A. Wahab, I. Kim, and C. S. Ha, "Bridged amine-functionalized mesoporous organosilica materials from 1,2-bis(triethoxysilyl)ethane and bis[(3-trimethoxysilyl)propyl]amine," J. Solid State Chem., vol. 177, no. 10, pp. 3439-3447, 2004.

[23] M. Dasog, Z. Yang, S. Regli, T. M. Atkins, A. Faramus, M. P. Singh, E. Muthuswamy, S. M. Kauzlarich, R. D. Tilley, and J. G. C. Veinot, "Chemical insight into the origin of red and blue photoluminescence arising from freestanding silicon nanocrystals," ACS Nano, vol. 7, pp. 2676-2685, 2013.

[24] R. a. Bley, S. M. Kauzlarich, H. W. H. Lee, and J. E. Davis, "Characterization of Silicon Nanoparticles Prepared from Porous Silicon," MRS Proc., vol. 351, no. 13, pp. 1881-1888, 1994.

[25] R. J. Clark, M. K. M. Dang, and J. G. C. Veinot, "Exploration of organic acid chain length on watersoluble silicon quantum dot surfaces," Langmuir, vol. 26, no. 19, pp. 15657-15664, 2010.

[26] B. P. Payne, M. C. Biesinger, and N. S. Mclntyre, "X-ray photoelectron spectroscopy studies of reactions on chromium metal and chromium oxide surfaces," J. Electron Spectros. Relat. Phenomena, vol. 184, no. 1-2, pp. 29-37, 2011.

[27] M. National Institute of Standards and Technology: Gaithersburg, "NIST X-ray Photoelectron Spectroscopy Database, Version 4.1," 2012. [Online]. Available: http://srdata.nist.gov/xps/.

[28] L. Feng, L. He, Y. Ma, and Y. Wang, "Grafting poly(methyl methacrylate) onto silica nanoparticle surfaces via a facile esterification reaction," Mater. Chem. Phys., vol. 116, pp. 158-163, 2009.

[29] J. J. Romero, M. J. Llansola-portole, D. Arciprete, B. Rodr, A. L. Moore, and C. Gonzalez, "Photoluminescent 1 - 2 nm Sized Silicon Nanoparticles: A Surface- Dependent System," Chem. Mater., 2013.

[30] M. F. L. de M. and M. C. G. Damián Rodríguez Sartori, Cristian R Lillo, Juan J Romero, María Laura 
Dell'Arciprete, Alejandro Miñán, "Polyethylene glycol-coated blue-emitting silicon dots with improved properties for uses in aqueous and biological environments," Nanotechnology, vol. 27, no. 47, pp. 111.

[31] J. J. Romero, M. Wegmann, C. Lillo, A. Rubert, S. Klein, and C. Kryschi, "Impact of Iron Incorporation on 2 - 4 nm Size Silicon Nanoparticles Properties," 2015.

[32] J. F. Moulder, W. F. Stickle, P. E. Sobol, and K. D. Bomben, "Handbook of X-ray Photoelectron Spectroscopy," Surface And Interface Analysis, vol. 3, no. 4. pp. v-v, 1979.

[33] "Si environment XPS AES.pdf." .

[34] P. Caregnato, M. D. E. Forbes, D. B. Soria, D. O. Mártire, and M. C. Gonzalez, "Chemisorbed thiols on silica particles: Characterization of reactive sulfur species," J. Phys. Chem. C, vol. 114, pp. 50805087, 2010.

[35] S. Klein, M. L. Dell'Arciprete, M. Wegmann, L. V. R. Distel, W. Neuhuber, M. C. Gonzalez, and C. Kryschi, "Oxidized silicon nanoparticles for radiosensitization of cancer and tissue cells," Biochem. Biophys. Res. Commun., vol. 434, pp. 217-222, 2013.

[36] K. limura, N. Suzuki, and T. Kato, “10.1246@bcsj.69.1201.pdf," Bull. Chem. Soc. Jpn., vol. 69, pp. 1201-1211, 1996.

[37] E. J. Henderson, J. a. Kelly, and J. G. C. Veinot, "Influence of HSiO1.5 sol-gel polymer structure and composition on the size and luminescent properties of silicon nanocrystals," Chem. Mater., vol. 21, no. 10, pp. 5426-5434, 2009.

[38] Y. Kanemitsu, S. Okamoto, M. Otobe, and S. Oda, "Photoluminescence mechanism in surfaceoxidized silicon nanocrystals," Phys. Rev. B, vol. 55, no. 12, pp. R7375-R7378, 1997.

[39] J. Diener, M. Ben-Chorin, D. . Kovalev, S. . Ganichev, and F. Koch, "Excitation of the porous silicon photoluminescence by a multiphoton vibronic process," Thin Solid Films, vol. 276, pp. 116-119, 1996.

[40] J. Fuzell, A. Thibert, T. M. Atkins, M. Dasog, E. Busby, J. G. C. Veinot, S. M. Kauzlarich, and D. S. Larsen, "Red states versus blue states in colloidal silicon nanocrystals: Exciton sequestration into lowdensity traps," J. Phys. Chem. Lett., vol. 4, pp. 3806-3812, 2013.

[41] C. Cimpean, V. Groenewegen, V. Kuntermann, A. Sommer, and C. Kryschi, "Ultrafast exciton relaxation dynamics in silicon quantum dots," Laser Photonics Rev., vol. 3, no. 1, pp. 138-145, 2009.

[42] V. Kuntermann, C. Cimpean, G. Brehm, G. Sauer, C. Kryschi, and H. Wiggers, "Femtosecond transient absorption spectroscopy of silanized silicon quantum dots," Phys. Rev. B, vol. 77, pp. 1-8, 2008.

[43] J. Martin, F. Cichos, F. Huisken, and C. Von Borczyskowski, "Electron-phonon coupling and localization of excitons in single silicon nanocrystals," Nano Lett., vol. 8, pp. 656-660, 2008.

[44] F. Cichos, C. von Borczyskowski, and M. Orrit, "Power-law intermittency of single emitters," Curr. Opin. Colloid Interface Sci., vol. 12, pp. 272-284, 2007.

[45] H. Von, "Photoinduced Processes in Silicon Nanoparticles," vol. 44, 2004. 
- 114 - 


\section{Capítulo 6: Regulación de propiedades ópticas.}

\section{Díadas empleando colorantes orgánicos}

En el presente capítulo se detalla la síntesis, caracterización y estudio de propiedades fotofísicas de díadas soportadas sobre Si nps. Se emplearon 3 familias de colorantes orgánicos: Porfirinas, carotenoides y dicetopirrolopirroles. Estas familias de colorantes fueron elegidas particularmente debido a su alto potencial de oxidación en el estado excitado, a fin de sintetizar díadas que soporten procesos de transferencia de carga. El uso de los colorantes orgánicos puede variar según la díada sintetizada: En el caso de porfirinas y dicetopirrolopirroles funcionó como cromóforo principal y donor de carga, mientras que las Si nps se emplearon como aceptoras de carga. En el caso de carotenoides, se usaron también como cromóforo principal y donor de carga, pero también podrían usarse como potenciales aceptores de energía por vía no radiativa. Se estudió en detalle la dinámica de los estados excitados generados, prestando especial atención a estados de transferencia de carga. El fin último de esta parte del trabajo es el desarrollo de estados de transferencia de carga de tiempos de vida largos, para ser empleados tanto en procesos de absorción y separación de carga en conversión de energía o como sensibilizadores de la producción de especies reactivas.

\subsection{Introducción}

El proceso de separación de cargas es uno de los pasos más importantes de la fotosíntesis, involucrado en la transformación de energía solar en potencial químico. Esta estrategia inspiró distintos enfoques que hoy en día se denominan fotosíntesis artificial [1]. Estos involucran el diseño y ensamblado de sistemas capaces de producir combustibles solares, celdas de combustible fotoelectroquímicas, e ingeniería de enzimas de organismos fotoautotróficos. A su vez, la nanotecnología contribuyó a este campo con nuevos materiales activos para conversión de energía y buscando el re-diseño de sistemas para aumentar su eficiencia [2]. En línea con esta idea, en este capítulo reportamos el diseño, síntesis y propiedades fotofísicas de nuevas díadas usando Si nps y cromóforos orgánicos, a fin de obtener estados de transferencia de carga estables.

Sintetizamos Si nps de distintos tamaños usando la oxidación térmica de siliciuros metálicos [3] y las funcionalizamos con alilamina vía hidrosililación. Luego de esto, las Si nps amino terminales reaccionaron con derivados carboxilados de tetra-mesitil porfirina, carotenoides o dicetopirrolopirroles (dpps). Las Si nps fueron caracterizadas usando XPS y HR-TEM, mientras que las díadas fueron estudiadas usando FTIR y espectroscopías UV-vis de absorción y emisión, tanto estacionarias como resueltas en el tiempo.

Analizamos los resultados obtenidos en base a la teoría de Marcus de reacciones de transferencia de carga. La constante de transferencia de electrones $\left(\mathrm{k}_{\mathrm{e}}\right)$ depende del tamaño de las Si nps usadas y están fuertemente influenciadas por la estrategia de unión empleada, reflejando el efecto del acoplamiento electrónico.

\subsection{Aspectos sintéticos y metodológicos}

Los detalles referidos a síntesis y métodos experimentales han sido desarrollados en mayor extensión en los capítulos 2 y 3 . A continuación, se comentarán de forma breve los procedimientos relacionados al presente capítulo.

Las Si nps estudiadas en este capítulo fueron sintetizadas usando una adaptación de las técnicas publicadas por Kauzlarich y colaboradores [3][4][5]. Los detalles correspondientes a esta síntesis se encuentran en las secciones 3.2.4 a 3.2.7. del capítulo 3. A su vez, la obtención, caracterización y propiedades ópticas de PA-BU' 
Si nps de distintos tamaños se detalla en el capítulo 5. Por simpleza conservaremos la nomenclatura utilizada en el capítulo 5 para las PA-BU' Si nps de distintos tamaños (notación de Sj), en la cual llamamos S1 a las Si nps de $3.6 \mathrm{~nm}, \mathrm{~S} 3$ a las de $4 \mathrm{~nm}$ y S4 a las de $5 \mathrm{~nm}$.

Brevemente, en un balón de 3 bocas con un refrigerante adosado, se colocaron $120.00 \mathrm{ml}$ de DMF anhidro, que fueron calentados hasta $100^{\circ} \mathrm{C}$ y purgados con Ar durante $30 \mathrm{~min}$. Luego, se agregaron $100.0 \mathrm{mg} \mathrm{de} \mathrm{Mg}_{2} \mathrm{Si}$ finamente molido y el sistema se mantuvo en calentamiento y purga hasta que alcance el reflujo. En el paso siguiente, se adicionó al sistema una cantidad controlada de $\mathrm{NH}_{4} \mathrm{Cl}$ disueltos en $10.00 \mathrm{ml}$ de DMF y se continuó el calentamiento hasta la condición de reflujo, que se mantuvo por $20 \mathrm{hs}$. Según la cantidad utilizada de $\mathrm{NH}_{4} \mathrm{Cl}$ se obtuvieron BU' Si nps de distintos tamaños, como se explica y detalla en el capítulo 5 . Finalmente, el sistema se dejó enfriar a temperatura ambiente. En estas condiciones se obtuvieron BU' Si nps de tamaño variable, las cuales fueron purificadas usando diálisis. Sobre la suspensión obtenida se agregaron $250 \mu \mathrm{l}$ de AA y $500 \mu \mathrm{l}$ de una solución de $\mathrm{H}_{2} \mathrm{PtCl}_{6} 5 \mathrm{mM}$ en $\mathrm{MeOH}$, luego la muestra se calentó hasta llegar a reflujo y se purgó usando Ar. Se mantuvo el reflujo por ca. $20 \mathrm{hs}$ para luego dejar enfriar a temperatura ambiente. Finalmente, el solvente se removió evaporando a presión reducida y la muestra se redisolvió en $25.00 \mathrm{ml}$ del solvente de trabajo.

La unión de los distintos colorantes se realizó a través de enlaces amida, entre derivados carboxilados de los colorantes y Si nps cubiertas con 2-propenilamina (PA-BU' Si nps). La formación de enlaces amida procede a través de dos pasos sintéticos: Un paso de activación donde se forma un compuesto de acilo reactivo, y posteriormente una aminólisis donde el acilo reactivo es atacado por la amina a unir. Existen diversos reactivos los cuales forman compuestos acilos reactivos, en esta tesis se usaron en particular diciclohexilcarbodiimida (DCC) junto a N-hidroxisuccinimida (NHS), o cloruro de oxalilo. Para cada caso se empleó una estrategia particular, considerando las distintas condiciones de reacción y los subproductos formados.

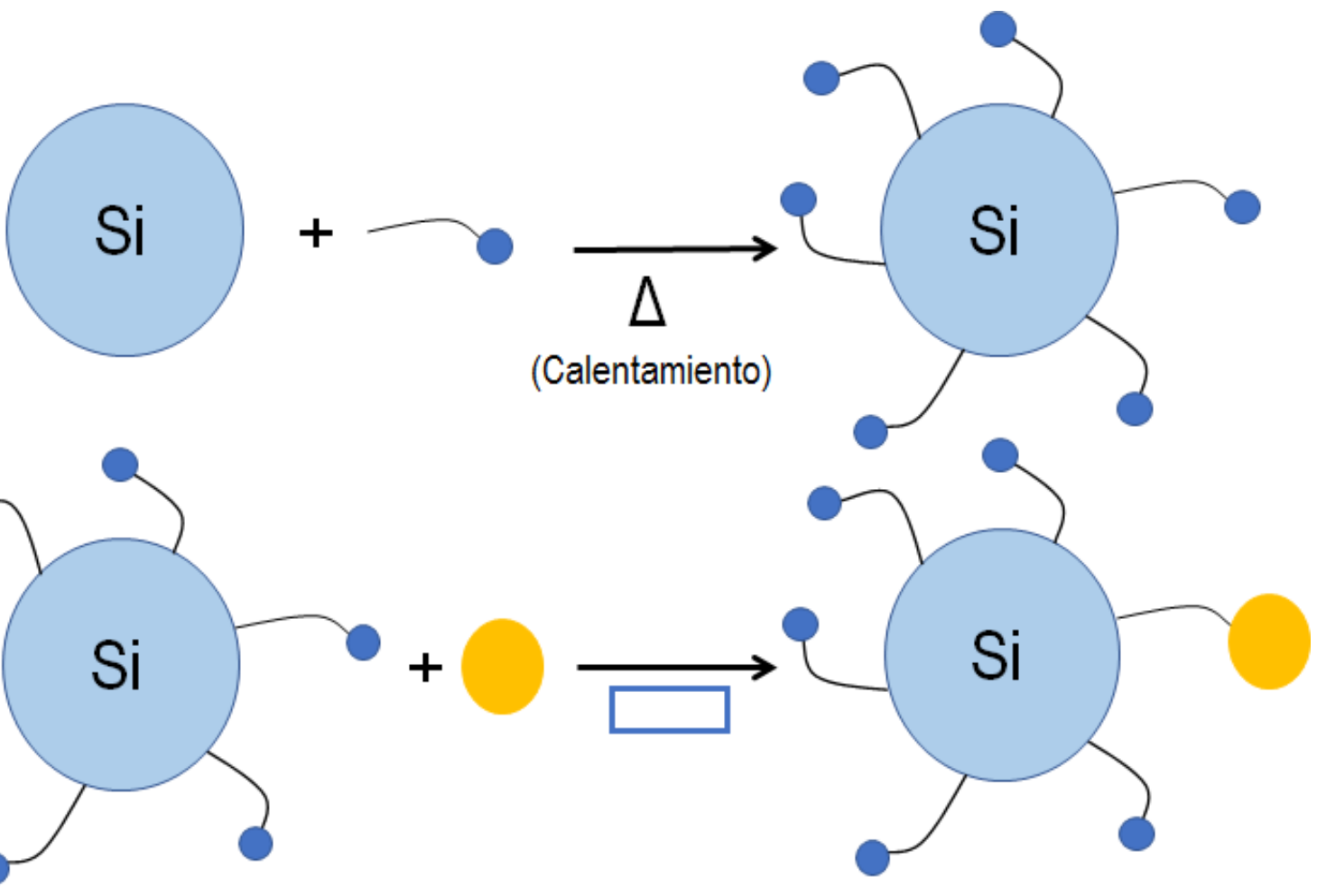

Figura 6.1. Esquema de síntesis de PA-BU' Si nps y su posterior acoplamiento a cromóforos de interés (Carotenoides, porfirinas y dicetopirrolopirroles). El recuadro celeste simboliza las distintas condiciones y kits de unión usados.

Para las díadas con profirinas y carotenoides se emplearon DCC y NHS. Se mezcló en $10.00 \mathrm{ml}$ deDMF seco el precursor ácido con DCC y NHS en exceso ( $n_{\text {соон: }} n_{D C C}$ de 1:1.5) y se dejó en agitación suave por 12 hs en 
oscuridad, en atmósfera inerte de Ar y a temperatura ambiente. Luego de esto, se agregó cantidad necesaria de las Si nps aminadas en suspensión de DMF manteniendo la relación $n_{\text {соон: }} n_{\text {si nps }}$ cercano a 1:1, y se mantuvo la agitación en las mismas condiciones por 12 hs más. Finalmente, el subproducto formado (diciclohexilurea, DCU) puede removerse por filtración, ya que forma un precipitado insoluble. Es importante notar que los carotenoides y las díadas que los contienen son muy sensibles a las condiciones ambientales, ya sea a la atmósfera gaseosa o de iluminación, por lo que su uso y estudio es una tarea complicada.

En el caso de los dpps, las reacciones de formación de enlaces amida se realizaron usando cloruro de oxalilo. se llevaron a cabo en condiciones similares a las de DCC/NHS, con diferencias al momento de la purificación. El precursor ácido y el cloruro de oxalilo (exceso de 1:1.5) fueron disueltos en $10.00 \mathrm{ml}$ de DMF seco y mezclados por agitación durante 12 hs en oscuridad, en atmósfera inerte de Ar y a temperatura ambiente. Luego se colocaron las Si nps aminadas en suspensión de DMF en cantidad necesaria para garantizar $n_{\text {cooH: }} n_{\text {Si nps }}$ cercano a 1:1. y se mantuvo la agitación en las mismas condiciones por 12 hs más. Como los subproductos en este caso son gases, el proceso de aislamiento y purificación fue más simple que en el caso de porfirinas 0 carotenoides.

Para corroborar el avance de la reacción y la pureza de la muestra final, se empleó cromatografía en capa fina. A su vez, el proceso de diálisis no fue del todo efectivo para remover el colorante en exceso presente, por lo cual usamos cormatografía de permeación en geles o de exclusión molecular (GPC, del inglés Gel Permeation Chromatography) como un paso preparativo previo de purificación. Para esto, preparamos el lecho cromatográfico usando sephadex G-25 @ hinchado por 24 hs con DMF seco, como se sugiere en bibliografía [6]. De todos modos, dentro de las distintas variantes empleadas, tal como se describirá más adelante, siempre quedó una pequeña fracción remanente. A su vez, no puede descartarse la adsorción inespecífica de los colorantes ya que toma un papel muy importante al momento de analizar los datos. Desde corrimientos espectrales [7] hasta sutiles cambios en los tiempos de vida [8], el efecto de la superficie sobre los colorantes es diverso y particular para cada uno [9][10][11].

Los colorantes usados son de la familia de las porfirinas, carotenoides y dicetopirrolopirroles. De la primera, se usaron derivados monocarboxilados de la tetra-mesitil porfirina, tanto libre (TMP, denominada 1 en este capítulo) como con un átomo de Zn ligado al centro de N ((Zn)TMP, llamada 1'). En el caso de carotenoides, se emplearon derivados monocarboxilados de carotenos con 9, 10 y 11 dobles enlaces (C9, C10 y C11 respectivamente). El dicetopirrolopirrol empleado es un derivado asimétrico, con un grupo octilo y un grupo carboxilo en cada $\mathrm{N}$ del núcleo pirrolopirrol (dpp). Para facilitar la comprensión, mostramos figuras de los colorantes empleados. Estos colorantes fueron cedidos amablemente por los grupos de los profesores Dra. Moore, Dr. Moore y Dr. Gust (en el caso de porfirinas y carotenoides) y del Prof. Dr. Vullev (dicetopirrolopirroles). 


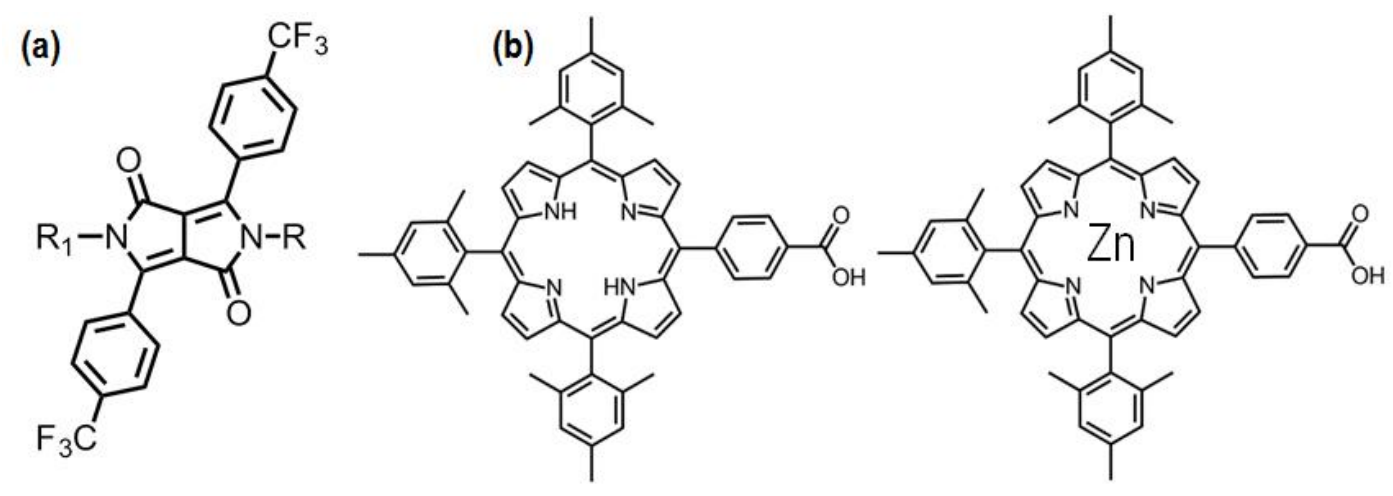<smiles>CC1=C(/C=C/C(C)=C/C=C/C(C)=C/C=C/C=C(C)/C=C/C=C(\C)C(=O)O)C(C)(Cl)CCC1</smiles><smiles>CC1=C(/C=C/C(C)=C/C=C/C(C)=C/C=C/C=C(C)/C=C/C=C(C)/C=C/C(=O)O)C(C)(C)CCC1</smiles>

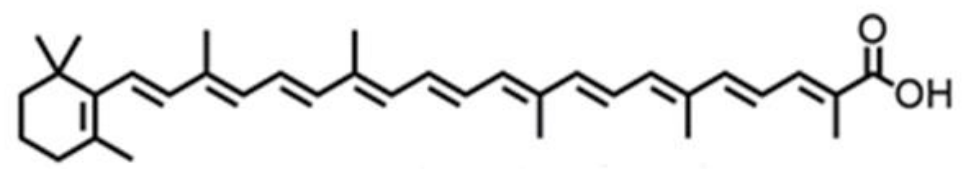

Figura 6.2. Colorantes empleados en este capítulo. (a) DPP, el empleado en esta tesis presenta $\mathrm{R}_{1}=-\mathrm{COOH}$ y $\mathrm{R}=-\mathrm{C}_{8} \mathrm{H}_{17}$, (b) derivados carboxilados de tetra-mesitil porfirina libre y con $\mathrm{Zn}^{2+}$, (c) derivados carboxilados del caroteno con 9,10 y 11 dobles enlaces.

Finalmente, usamos el siguiente esquema para nombrar las díadas preparadas usando los distintos colorantes.

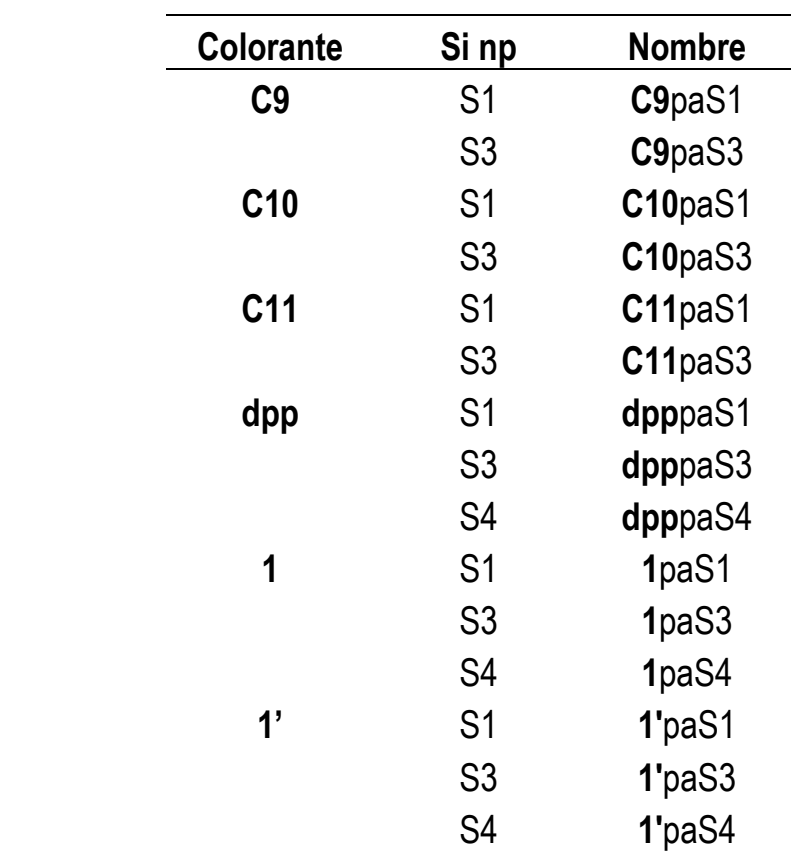

Tabla 6.1. Nomenclatura empleada para las díadas estudiadas en este capítulo. 


\subsection{Resultados y Discusión}

\subsubsection{Síntesis y caracterización}

Las PA-BU' Si nps usadas en estos experimentos fueron caracterizadas de forma exhaustiva, y dichos resultados se encuentran presentes en el capítulo 5. Luego, hicimos reaccionar las PA-BU' Si nps con distintos derivados carboxilados de los colorantes de interés en presencia de moléculas que catalicen la reacción de formación del enlace amida. Si bien los enlaces amida presentan diversas ventajas, como por ejemplo un amplio repertorio de condiciones y catalizadores para realizar la reacción, estereoquímica definida y alta estabilidad [12]; también pueden actuar como trampas de electrones en reacciones de transferencia de carga si el potencial de reducción del aceptor está en el orden del valor característico de amidas o en caso de restricciones de tipo cinético [13].

Si bien la estrategia sintética de las díadas no fue compleja y usó reacciones muy robustas, el aislamiento y purificación fue complicado. En primer lugar, intentamos usar diálisis para remover el exceso de colorantes. Al observar los espectros UV-vis del producto crudo con respecto al dializado se observó una mejora, pero nunca suficiente para proseguir con los estudios espectroscópicos. A tal fin, utilizamos GPC sobre la muestra dializada para obtener una mejor separación, la cual fue variable según sea el colorante a separar. La muestra ingresó al lecho cromatográfico en el menor volumen posible, y se eluyó lentamente usando DMF, a fin de separar en el mayor grado posible el producto. El avance de la corrida en las distintas fracciones se corroboró por TLC, usando distintas fases móviles para cada cromóforo (acetato de etilo para porfirinas y carotenoides, y diclorometano : metanol 9:1 para dpp).

Para corroborar la formación del enlace amida y en consecuencia, de la díada propuesta, tomamos espectros FTIR de las distintas díadas preparadas. Además de las señales típicas de los colorantes empleados [14][16][17]-[19][20]y las PA - BU' Si nps [21][22], observamos la presencia de señales entre 1630 a $1700 \mathrm{~cm}^{-1}$ características de amidas, y la disminución de las señales a $3300-3400 \mathrm{~cm}^{-1}$ de aminas primarias que cubren las Si nps. A su vez, observamos señales en $1720-1780 \mathrm{~cm}^{-1}$ debida a derivados carboxilados del cromóforo sin acoplar.

\subsubsection{Propiedades fotofísicas}

Una vez aisladas, estudiamos las díadas preparadas usando una batería de técnicas espectroscópicas tanto en estado estacionario como resueltas en el tiempo. El fin de estos experimentos, como así también de este capítulo, es obtener sistemas que puedan ser excitados usando radiación visible y generen estados electrónicos de utilidad en procesos de separación de carga o fotosensibilización, para mejorar las prestaciones de las Si nps en estas aplicaciones[23]. En todos los casos, estimamos la diferencia de energía libre para el proceso de transferencia de carga fotoinducida (PeT, del inglés Photoinduced electron Transfer) como recomienda la IUPAC [24]-[26]. Los potenciales redox de los estados excitados que utilizamos a lo largo del capítulo se resumen en la siguiente figura. 


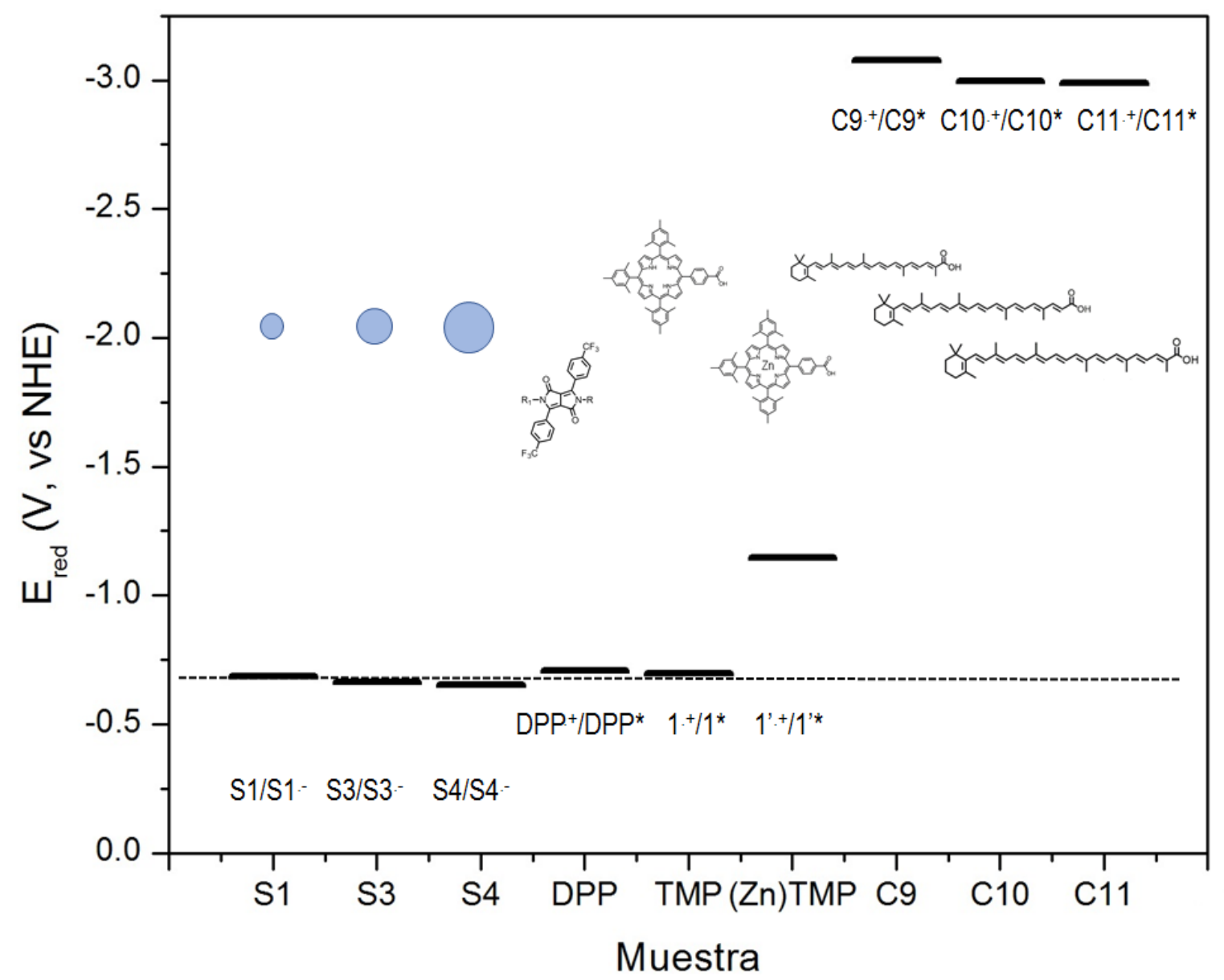

Figura 6.3. Potenciales de reducción para la CB de las PA-BU' Si np usadas en este capítulo, y para la reducción de cationes radicales de los colorantes empleados generando el primer estado excitado. Los valores citados para los colorantes fueron obtenidos de literatura [14], [27]-[30]. En línea punteada se muestra como referencia el valor de E para la CB de PA-BU' Si nps de 3.4 nm de diámetro.

Para mayor organización, presentamos los resultados según sea el colorante empleado en la díada estudiada.

\subsubsection{Díadas empleando Porfirinas: $1 \mathrm{paSj}$ y 1'paSj}

Para estudiar estas díadas empleamos técnicas de absorción UV-vis y de emisión tanto estacionarias como resueltas en el tiempo. Los espectros de emisión y atenuación normalizados se presentan a continuación. Por simpleza, en primer lugar presentamos los experimentos realizados con las díadas $1 \mathrm{paSj}$ y luego con 1'paSj. 


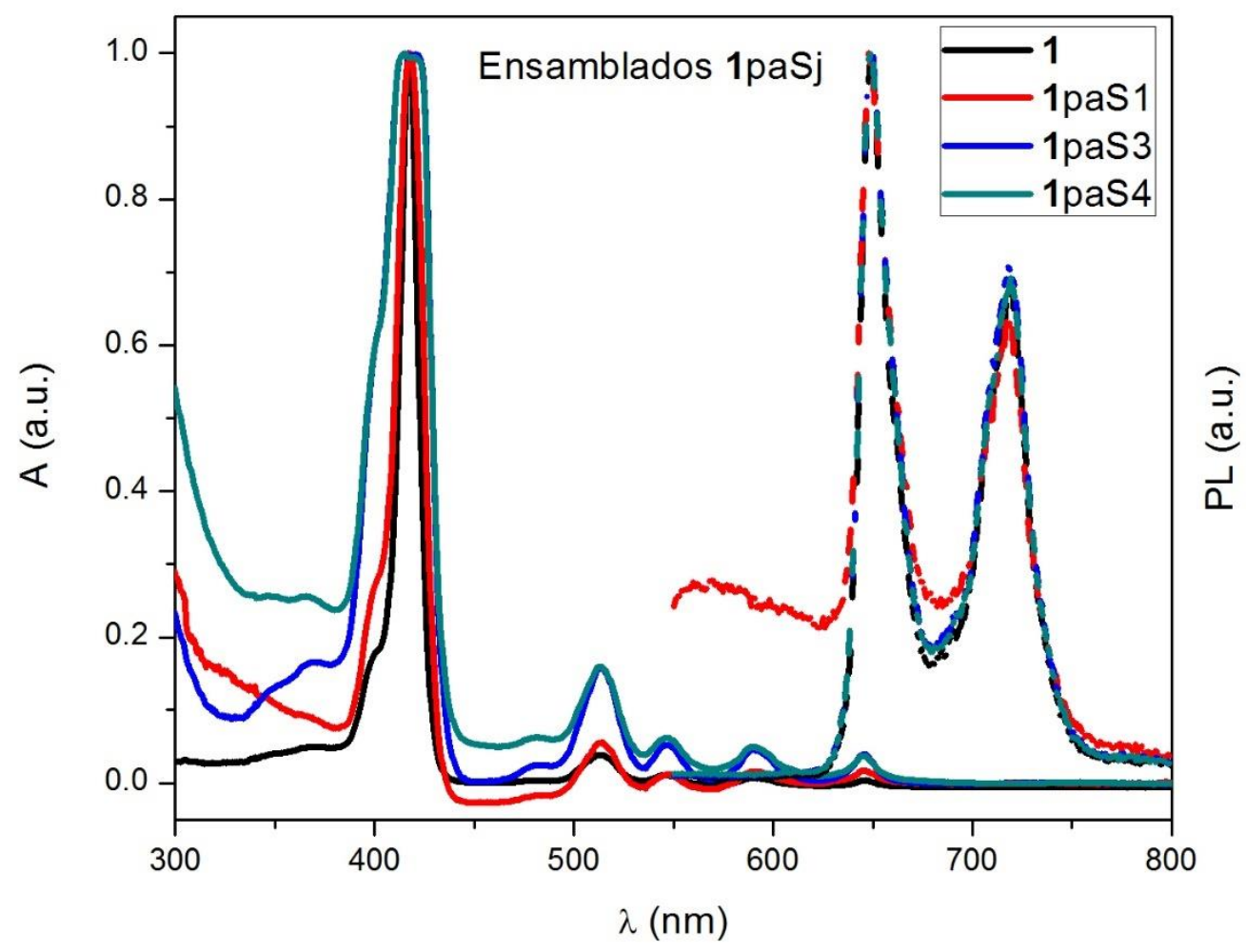

Figura 6.4. Espectros de atenuación (línea continua) y de emisión (línea cortada) normalizados para díadas 1paSj.

Medimos los espectros de absorción y emisión para las díadas empleando el colorante 1 tomando los cuidados necesarios, tal como se detalló en el capítulo 2, excitando estas muestras en particular a $\lambda_{\mathrm{ex}}=510 \mathrm{~nm}$. En estas condiciones, excitamos el colorante orgánico y estudiamos el efecto de las Si nps sobre sus vías de decaimiento. Pudimos apreciar tanto corrimientos espectrales como cambios en el ancho de las bandas con respecto al colorante libre, lo cual indica un cambio de las superficies de energía potencial tanto del estado excitado como del fundamental. Este comportamiento es esperable, debido al cambio en el entorno dieléctrico que experimentan las moléculas del colorante, que pasan de estar rodeadas por una caja de solvente a estar confinadas a una superficie, ya sea por unión covalente 0 adsorción inespecífica, fenómeno al cual las profirinas son muy proclives [9][31][11]. A su vez, si bien las condiciones sintéticas fueron elegidas para evitar la unión de múltiples molécuals de colorante sobre la misma nanopartícula, no puede descartarse la formación de dímeros [7]. Presentamos los espectros obtenidos en la figura 6.4 mientras que las posiciones observadas para las bandas se resumen en la tabla 6.2 . 


\begin{tabular}{|c|c|c|c|c|c|c|c|c|}
\hline \multirow[t]{2}{*}{ Muestra } & \multicolumn{6}{|c|}{$\lambda_{\text {uv vis }}(\mathrm{nm})$} & \multicolumn{2}{|c|}{$\lambda_{\mathrm{PL}}(\mathrm{nm})$} \\
\hline & \multicolumn{2}{|c|}{ Soret } & \multicolumn{4}{|c|}{$\mathbf{Q}$} & & \\
\hline 1 & 398.5 & 417 & 514 & 547 & 589.5 & 645.5 & 649 & 719 \\
\hline 1paS1 & 399 & 418 & 514.5 & 546 & 592 & 646 & 648 & 719 \\
\hline 1paS3 & 399.5 & 415.5 & 514.5 & 547 & 590 & 645.5 & 649 & 718 \\
\hline $1 \mathrm{paS4}$ & 399.5 & 415.5 & 513.5 & 547 & 589.5 & 645.5 & 649 & 719 \\
\hline
\end{tabular}

Tabla 6.2. Máximos de absorción y emisión para 1 y las díadas 1paSj. Se sombrean los valores para el colorante libre como referencia.

A su vez vimos que la intensidad de PL disminuyó al unir 1 con las Si nps, que por las propiedades redox del estado excitado $1^{*}$ y de las Si nps (estudiadas en el capítulo 5) es muy probable que esta disminución esté relacionada a procesos de transferencia de carga, o la formación de dímeros en la superficie. Para obtener más información sobre la dinámica del proceso, se realizaron experimentos de emisión resuelta en el tiempo ( $\lambda_{\mathrm{ex}}=$ $510 \mathrm{~nm}$ ), cuyos resultados se presentan en el gráfico 6.5 .
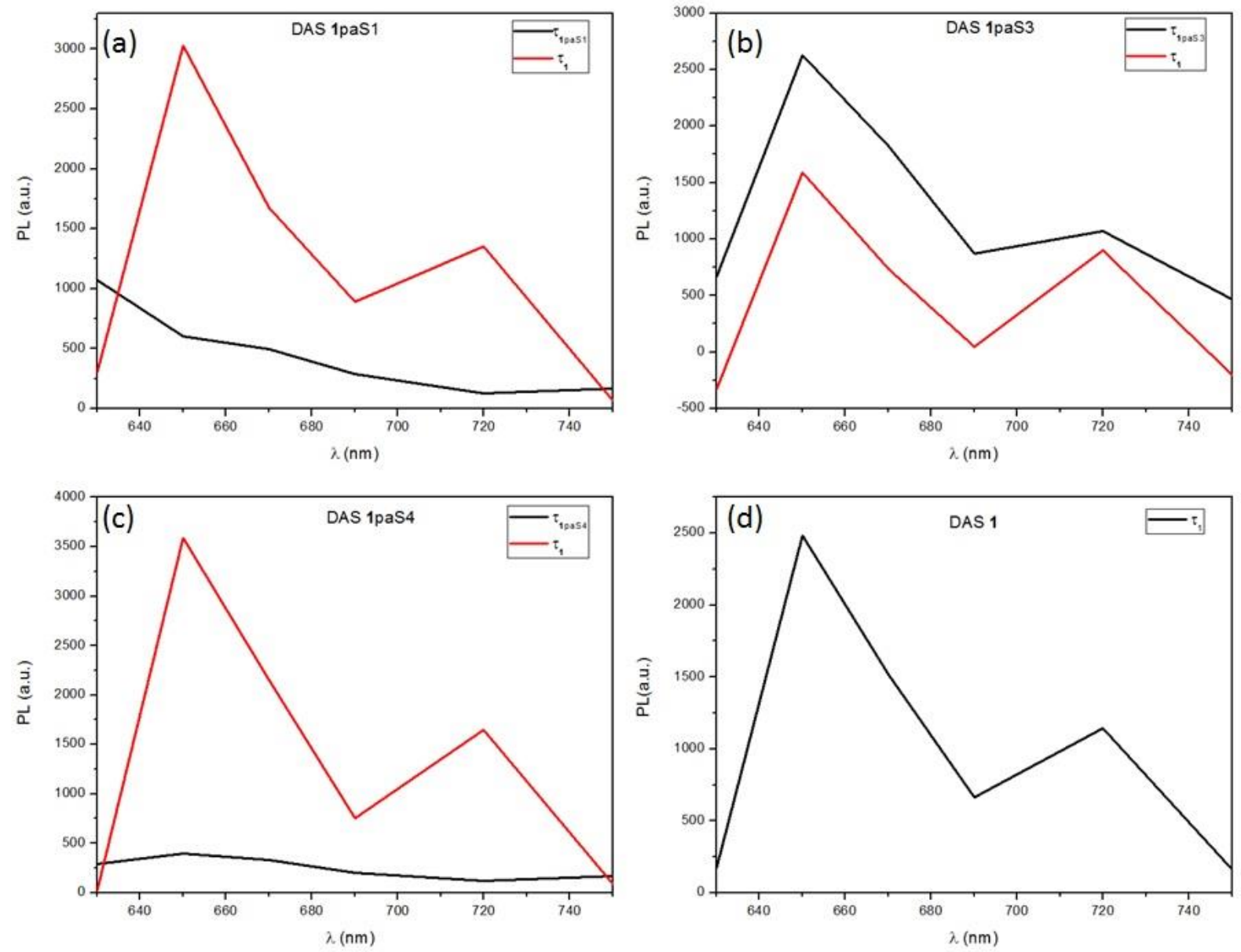

Figura 6.5. DAS obtenidos al analizar los datos de TRES para las díadas (a) 1paS1, (b) 1paS3, (c) 1 paS4 y (d) el colorante 1 libre.

Los datos de TRES se analizaron usando análisis global con un modelo de biexponencial de decaimientos paralelos, considerando que es posible que coexistan 1 libre 0 adsorbida sin unir de forma covalente (debido a 
los problemas de purificación mencionados) y 1 asociado en las díadas. Los DAS obtenidos muestran señales claras de la emisión desde 11 a tiempos largos $\left(\mathrm{T}_{1}\right)$, como así también una contribución extra a tiempos cortos que en principio podría entenderse como la emisión de 1 en la díada $\left(\mathrm{T}_{1 \mathrm{pas}} \mathrm{s}\right)$. Los DAS obtenidos están de acuerdo con lo reportado previamente en la bibliografía [14], [32], [33].

Considerando estos detalles obtuvimos buenos ajustes a los datos experimentales obtenidos, permitiendo determinar los tiempos de decaimiento asociado a cada especie. Este comportamiento bi exponencial puede deberse a o la existencia de estados que evolucionan dentro de la ventana temporal del experimento.

A su vez, si tenemos en cuenta que $\mathrm{T}=\left(\sum \mathrm{k}_{\mathrm{i}}\right)^{-1}$, donde $\mathrm{k}_{\mathrm{i}}$ es la constante de velocidad para el i-ésimo canal de desactivación y realizando la suma sobre todos los canales; asumimos que las constantes de velocidad para procesos que retornen el estado excitado sean mucho menores que $\mathrm{T}=\left(\sum \mathrm{k}_{\mathrm{i}}\right)^{-1}$, y que las constantes de velocidad para los canales presentes tanto en 1 como en las díadas no cambian, tenemos que:

$$
\left(\mathrm{T}_{1 \mathrm{paS}}\right)^{-1}-\left(\mathrm{T}_{1}\right)^{-1}=\mathrm{k}_{\mathrm{eT}}
$$

Si bien esta suposición suposición fuerte [1], [34]-[38] y no del todo correcta, ya que es esperable que el comportamiento del estado excitado cambie al pasar de la caja de solvente a la restricción en una posición fija superficie, permite obtener estimaciones para keT que nos permitan extraer conclusiones sobre el proceso de transferencia de carga, al menos en forma cualitativa. Los tiempos de decaimiento determinados, valores estimados de $\mathrm{k}_{\mathrm{eT}}, \Delta \mathrm{G}^{0}{ }_{\mathrm{PeT}}$ y los rendimientos cúanticos de $\mathrm{PL}$ excitando 1 libre 0 en las distintas díadas se presenta a continuación. En los datos medidos el error es menor 0 igual al $5 \%$, mientras que en el caso de las estimaciones es del $10 \%$.

\begin{tabular}{|c|c|c|c|c|c|}
\hline Muestra & $\Phi_{\mathrm{PL}} / \Phi_{\mathrm{PL}, 0}$ & T1paSj (ns) & $\mathrm{T}_{1}$ (ns) & $\Delta G^{\circ}{ }_{\mathrm{PeT}}(\mathbf{k j} / \mathrm{mol})$ & $k_{\mathrm{eT}}\left(p s^{-1}\right)$ \\
\hline 1 & 1 & & 10.91 & & \\
\hline $1 \mathrm{paS} 1$ & 0.41 & 3.16 & 10.76 & $-4.2 E+01$ & 0.00022 \\
\hline 1paS3 & 0.20 & 2.76 & 10.58 & $-3.8 \mathrm{E}+01$ & 0.00027 \\
\hline $1 \mathrm{paS} 4$ & 0.13 & 1.56 & 10.58 & $-3.2 E+01$ & 0.00055 \\
\hline
\end{tabular}

Tabla 6.3. Parámetros fotoquímicos medidos y estimados para las díadas $1 \mathrm{paSj}$. Se sombrean los valores para el colorante libre como referencia.

Es importante notar que los valores de los rendimientos cuánticos de emisión relativos al colorante libre son una cota superior al valor real, debido a la presencia de porfirinas adsorbidas inespecíficamente en la superficie, que presentan un rendimiento cuántico distinto a las porfirinas ligadas formando las díadas y a la libre. De todos modos, los valores determinados experimentalmente disminuyen a medida que aumenta $k_{e} T$, con lo cual la tendencia obtenida para $\mathrm{k}_{\mathrm{e}} \mathrm{u}$ usando la suposición (6.1) condice con la evidencia dentro del error experimental.

Los valores obtenidos para $\mathrm{k}_{\mathrm{e}}$ están de acuerdo con los reportados en bibliografía para sistemas formados por porfirinas usando aceptores orgánicos diversos [1], [32], [34], [39], [40]. A su vez, es importante notar que al aumentar $\Delta \mathrm{G}_{\mathrm{PeT}}^{\mathrm{T}}, \mathrm{K}_{\mathrm{e}}$ disminuye. Sin embargo, existe poca información en bibliografía sobre energías de reorganización en superficies como las que presentan las Si nps empleadas en este capítulo [34], [35], con lo cual no es conveniente realizar un análisis mucho más profundo por ahora. Sin embargo, evaluaremos la relación entre $\Delta \mathrm{G}^{\circ}{ }_{\mathrm{PeT}}$ y $\mathrm{k}_{\mathrm{e}}$ para distintos colorantes soportados en estas superficies más adelante en este capítulo

Realizamos un estudio similar con las díadas empleando la porfirina 1', cuyo potencial de oxidación en el estado excitado es mayor que el que presenta 1. Los espectros de atenuación y emisión estacionarios (excitando a 
$\lambda_{\mathrm{ex}}=560 \mathrm{~nm}$ ) se presentan en la figura 6.5, mientras que la posición de los máximos de absorción y emisión se listan en la tabla 6.4 .

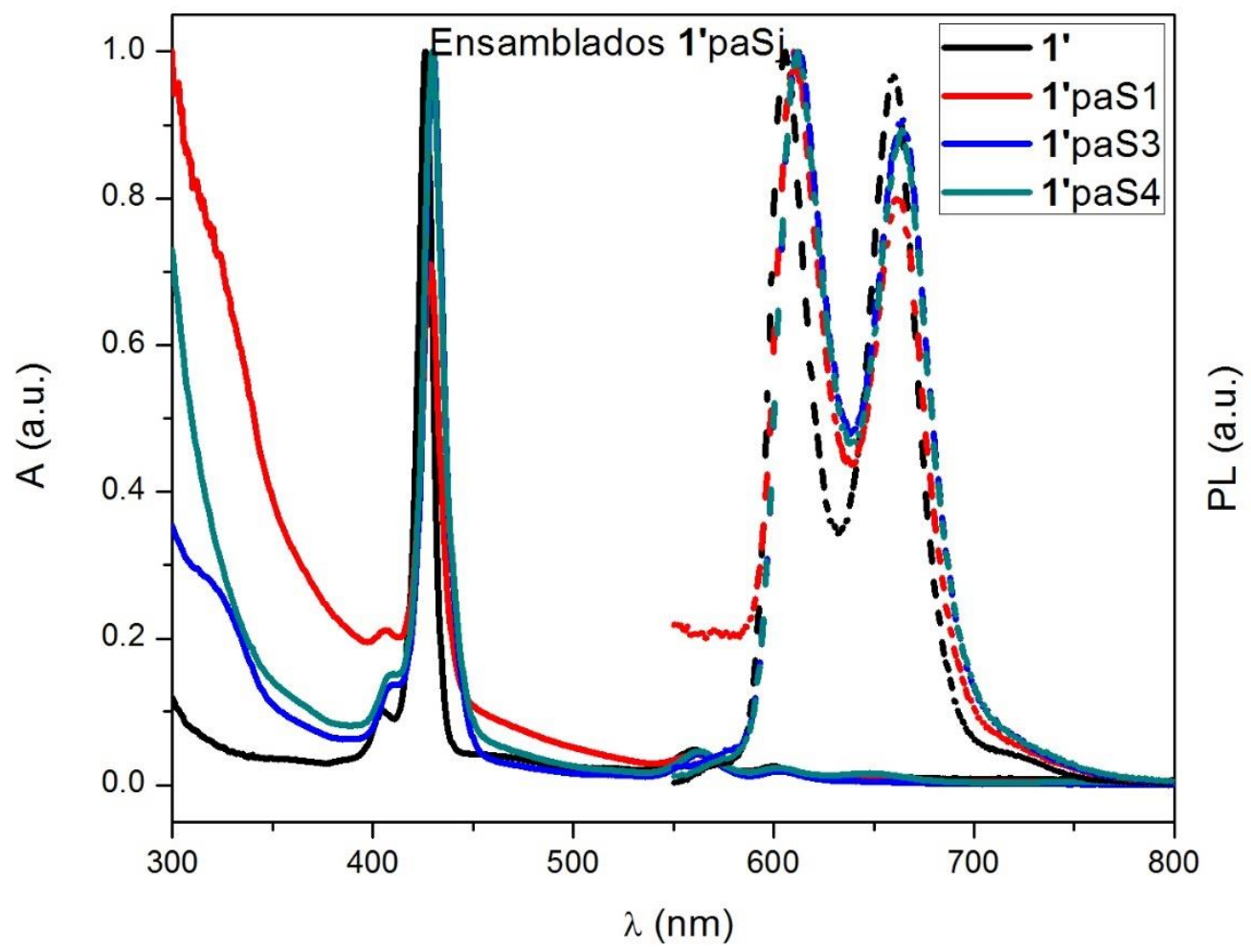

Figura 6.5. Espectros de atenuación (línea continua) y de emisión (línea cortada) normalizados para díadas 1’paSj.

Al igual que en el caso de las díadas usando 1, los cambios en la posición de los máximos indica una variación en las superficies de energía potencial del estado excitado y el fundamental, y muestran el efecto de la superficie en la estructura electrónica del colorante orgánico ya sea por unión covalente o adsorción inespecífica. A su vez, si bien las condiciones sintéticas fueron elegidas para evitar la unión de múltiples molécuals de colorante sobre la misma nanopartícula, no puede descartarse la formación de dímeros.

\begin{tabular}{|c|c|c|c|c|c|c|c|}
\hline \multirow[t]{2}{*}{ Muestra } & \multicolumn{5}{|c|}{$\lambda_{\text {uv vis }}(\mathrm{nm})$} & \multicolumn{2}{|c|}{$\lambda_{\mathrm{PL}}(\mathrm{nm})$} \\
\hline & \multicolumn{2}{|c|}{ Soret } & \multicolumn{3}{|c|}{$Q$} & & \\
\hline $1^{\prime}$ & 405 & 426.5 & 560 & 599.5 & 651 & 606 & 661 \\
\hline 1'paS1 & 406.5 & 428.5 & 561 & 601.5 & 648 & 610 & 661 \\
\hline 1'pas3 & 409 & 430 & 564 & 603.5 & 645.5 & 612 & 665 \\
\hline 1'paS4 & 408.5 & 429.5 & 563.5 & 604 & 648 & 612 & 664 \\
\hline
\end{tabular}

Tabla 6.4. Máximos de absorción y emisión para 1' y las díadas 1'paSj. Se sombrean los valores para el colorante libre como referencia. 
Del mismo modo que con las díadas sintetizadas con 1, se realizaron experimentos de emisión resuelta en el tiempo $\left(\lambda_{\mathrm{ex}}=560 \mathrm{~nm}\right)$. Usamos un modelo biexponencial de decaimientos paralelos para analizar los datos de TRES, que resultó satisfactorio y útil para obtener información sobre la transferencia de carga en este sistema. Los DAS obtenidos están en la figura 6.6. Aquí también los DAS muestran señales claras de la emisión desde 11' a tiempos similares al de la porfirina libre $\left(\mathrm{T}_{1}{ }^{\prime}\right)$, como así también una contribución a tiempos más cortos cuya forma es similar al espectro de PL de 1', pero con picos de mayor ancho, lo cual puede deberse a la influencia de la superficie sobre la estructura del estado $\left(\mathrm{T}^{\prime}{ }^{\prime} \mathrm{pas}\right)$. Los DAS obtenidos para las porfirinas en díadas están de acuerdo con lo reportado previamente en la bibliografía [14], [32], [33].

Una vez más usamos la ecuación (6.1) para estimar la constante de transferencia de electrones fotoinducida. En la tabla 6.5 presentamos los parámetros fotoquímicos estimados para estas díadas.
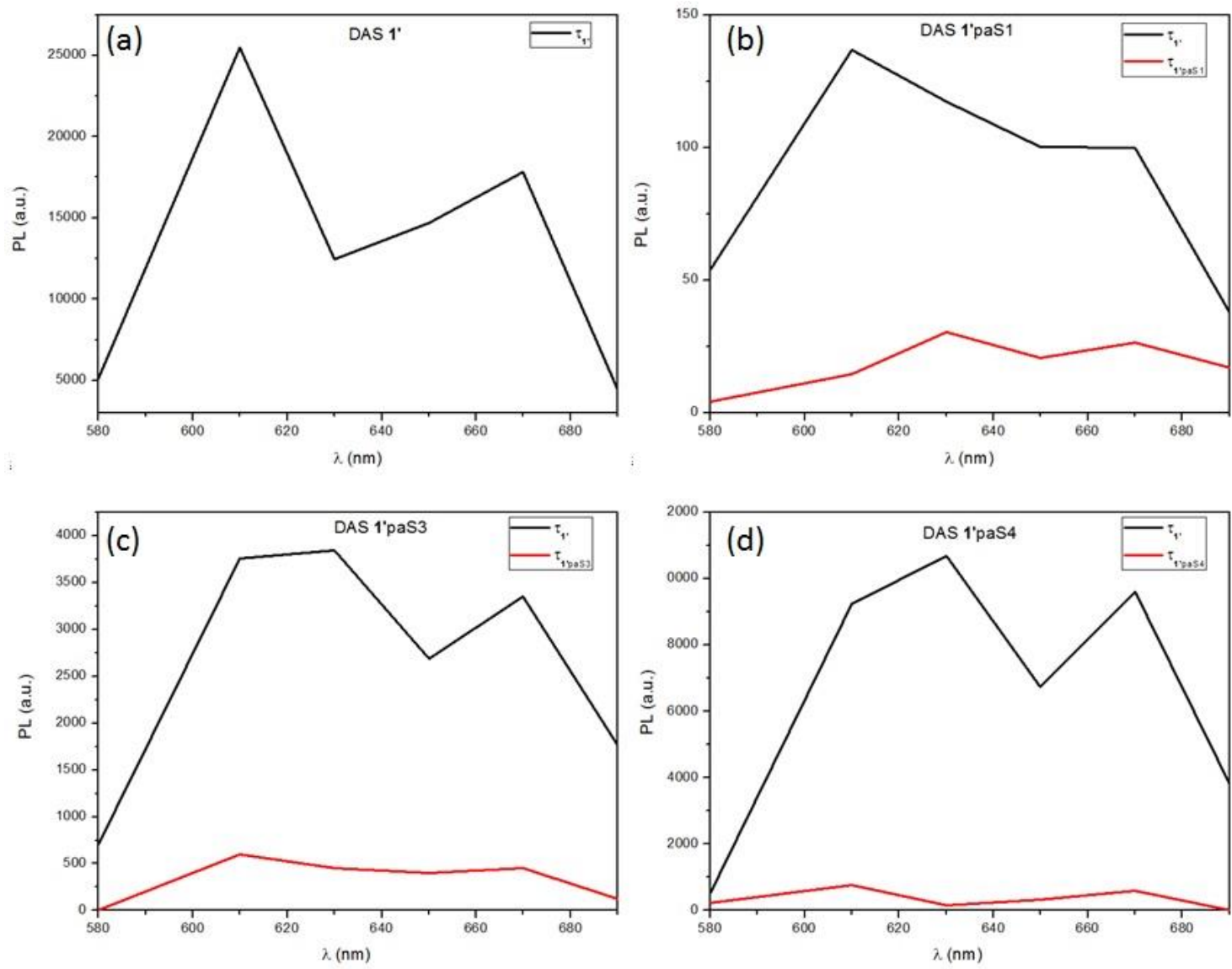

Figura 6.6. DAS obtenidos al analizar los datos de TRES para las díadas (a) el colorante 1' libre y las díadas (b) 1paS1, (c) 1paS3 y (d) 1paS4. Las condiciones de excitación se detallan en el texto.

\begin{tabular}{cccccc}
\hline Muestra & $\Phi_{\mathrm{PL}} / \Phi_{\mathrm{PL}, 0}$ & $\mathbf{T}_{1 \mathrm{paSj}}(\mathbf{n s})$ & $\mathbf{T}_{1}(\mathbf{n s})$ & $\Delta \mathbf{G}^{\circ} \mathrm{PeT}(\mathbf{k j} / \mathrm{mol})$ & $\mathbf{k}_{\mathrm{eT}}\left(\mathbf{p s}^{-1}\right)$ \\
\hline 1 1' & 1 & & 2.33 & & \\
1 'paS1 & 0.78312 & 0.71903132 & 2.90 & $-8.56 \mathrm{E}+01$ & 0.00010 \\
1 'paS3 & 0.68451 & 1.24036238 & 2.17 & $-8.14 \mathrm{E}+01$ & 0.00038 \\
1 'paS4 & 0.71819 & 1.39034876 & 2.18 & $-7.55 \mathrm{E}+01$ & 0.00029
\end{tabular}

Tabla 6.5. Parámetros fotoquímicos medidos y estimados para las díadas 1'paSj. Se sombrean los valores para el colorante libre como referencia. 
Es importante notar que, pese a que para las díadas 1 'paSj $\Delta G^{0}{ }_{\text {PeT }}$ es mayor que en el caso de $1 \mathrm{paSj}$, las constantes de velocidad no cambian de forma tan pronunciada. Este comportamiento puede estar ligado a la diferente energía de reorganización en los colorantes empleados, que es consecuencia de la distinta estructura química de los mismos. Situaciones similares se han reportado con otras porfirinas y clorinas que presentan $\mathrm{Zn}^{2+}$ como ion central con respecto al colorante básico [33], [41], [42]. Este hecho se ve reflejado también en la variación de los rendimientos cuánticos de emisión relativos, donde la variación es mínima. De todos modos, como se mencionó para las díadas $1 \mathrm{paSj}$, la presencia de colorante libre permite hacer una estimación superior al rendimiento cuántico real de emisión de la díada.

Por último, si consideramos la díada $\mathrm{D}$ podemos estimar el rendimiento cuántico de transferencia de carga en el proceso primario como $\Phi_{\mathrm{eT}, \mathrm{D}}=\mathrm{k}_{\mathrm{eT}, \mathrm{DT} \mathrm{D}}$. Los valores estimados para cada par donor - aceptor se presentan a continuación. Estos valores están en línea con lo discutido previamente.

\begin{tabular}{|c|c|c|c|c|}
\hline Díada & $\Delta G^{\circ}(\mathrm{kj} / \mathrm{mol})$ & $\mathrm{K}_{\mathrm{eT}, \mathrm{D}}\left(\mathrm{ns}^{-1}\right)$ & TD (ns) & $\Phi_{\mathrm{eT}, \mathrm{D}}$ \\
\hline 1paS1 & $-4.22 \mathrm{E}+01$ & 0.22 & 3.16 & 0.71 \\
\hline $1 \mathrm{paS} 3$ & $-3.80 E+01$ & 0.27 & 2.76 & 0.75 \\
\hline 1paS4 & $-3.20 \mathrm{E}+01$ & 0.55 & 1.56 & 0.86 \\
\hline 1'pas1 & $-8.56 \mathrm{E}+01$ & 0.96 & 0.72 & 0.69 \\
\hline 1'paS3 & $-8.14 E+01$ & 0.38 & 1.24 & 0.47 \\
\hline 1'paS4 & $-7.55 \mathrm{E}+01$ & 0.29 & 1.39 & 0.40 \\
\hline
\end{tabular}

Tabla 6.6. Parámetros fotoquímicos correspondientes al proceso de transferencia de carga fotoinducida en díadas 1paSj y 1'paSj.

A partir de los experimentos de PL que hicimos y los análisis que presentamos, el modelo cinético que mejor representa el sistema consta de 2 compartimentos que decaen de forma paralela (figura 6.7). Para obtener mayor información del sistema, sería útil realizar medidas de espectroscopía de absorción de transientes (TAS), a fin de obtener información de los estados no emisores, pero por inestabilidad de las muestras (precipitación) no pudieron realizarse. En consecuencia, no pudo estimarse $k_{\text {bet }}$ y $k_{c r}$. De todos modos, la evidencia experimental indica la formación de un estado de cargas separadas por excitación en el visible, logrando una ventaja sobre las Si nps sin cromóforos acoplados. 


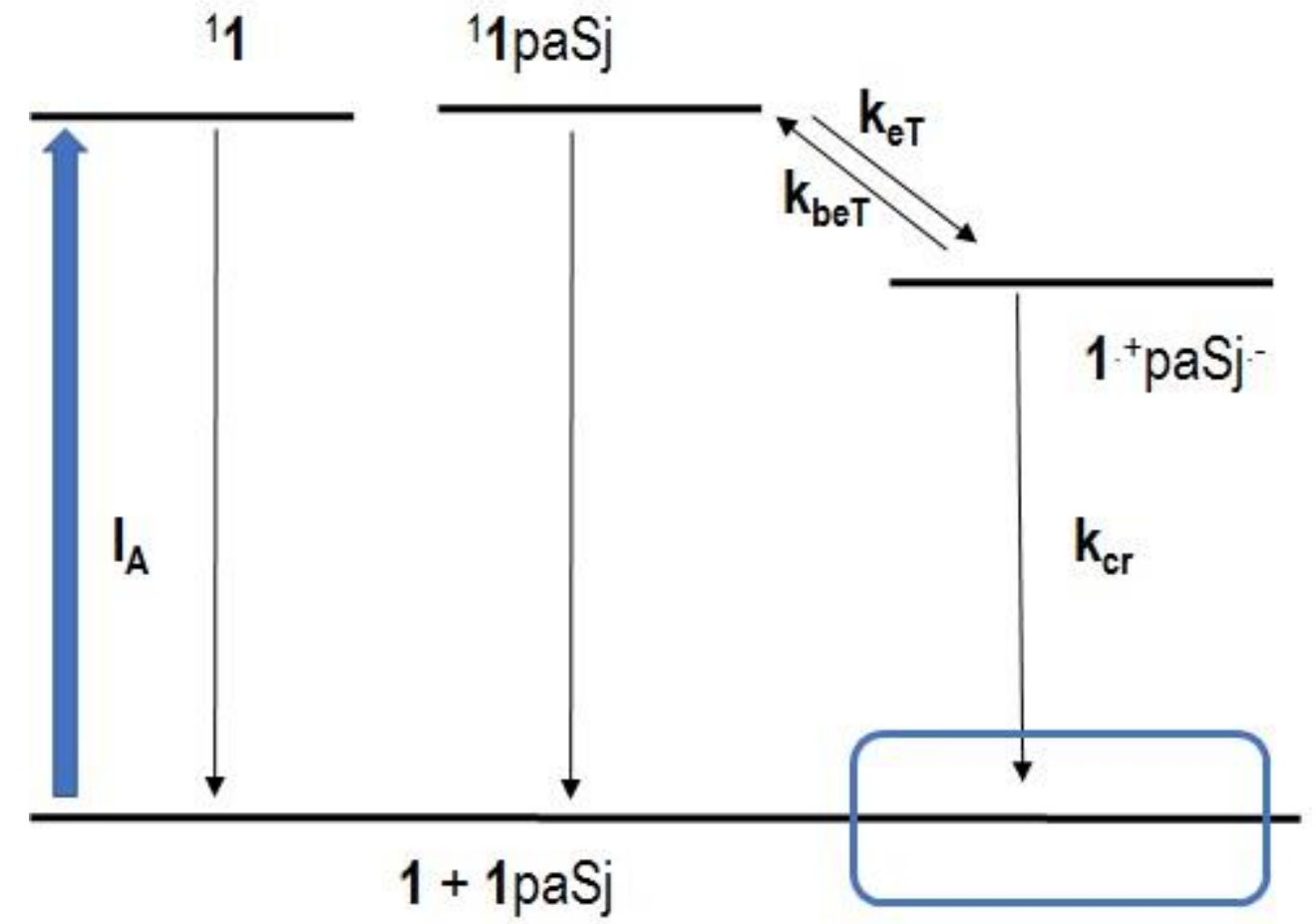

Figura 6.7. Esquema cinético empleado para el análisis de las medidas de TRES. El esquema propuesto es válido tanto para $1 \mathrm{paSj}$ como 1'paSj. Es importante notar que, usando técnicas de emisión, solo es posible detectar los compartimentos que emitan. El rectángulo celeste representa a los distintos estados de spin lo cuales pueden producirse por recombinación de carga y su posterior relajación. Para obtener información sobre los otros compartimentos y las constantes correspondientes es necesario usar TAS.

\subsubsection{Díadas empleando dpps: dpppaSj.}

El colorante dpp presenta un potencial de oxidación en el estado excitado intermedio entre 1 y 1 ', con el cual pretendimos explorar esa región de $\Delta G^{\circ}$ donde el valor que toma la energía de reorganización $\lambda$ es clave para determinar si el sistema se comporta según la forma normal o invertida en in diagrama de Marcus (keT vs $\Delta \mathrm{G}^{\circ}$ ). En primer lugar tomamos espectros de atenuación y emisión para las díadas, que presentamos en la figura 6.8. A su vez, en la tabla 6.7 presentamos las posiciones de los máximos de atenuación y emisión. 


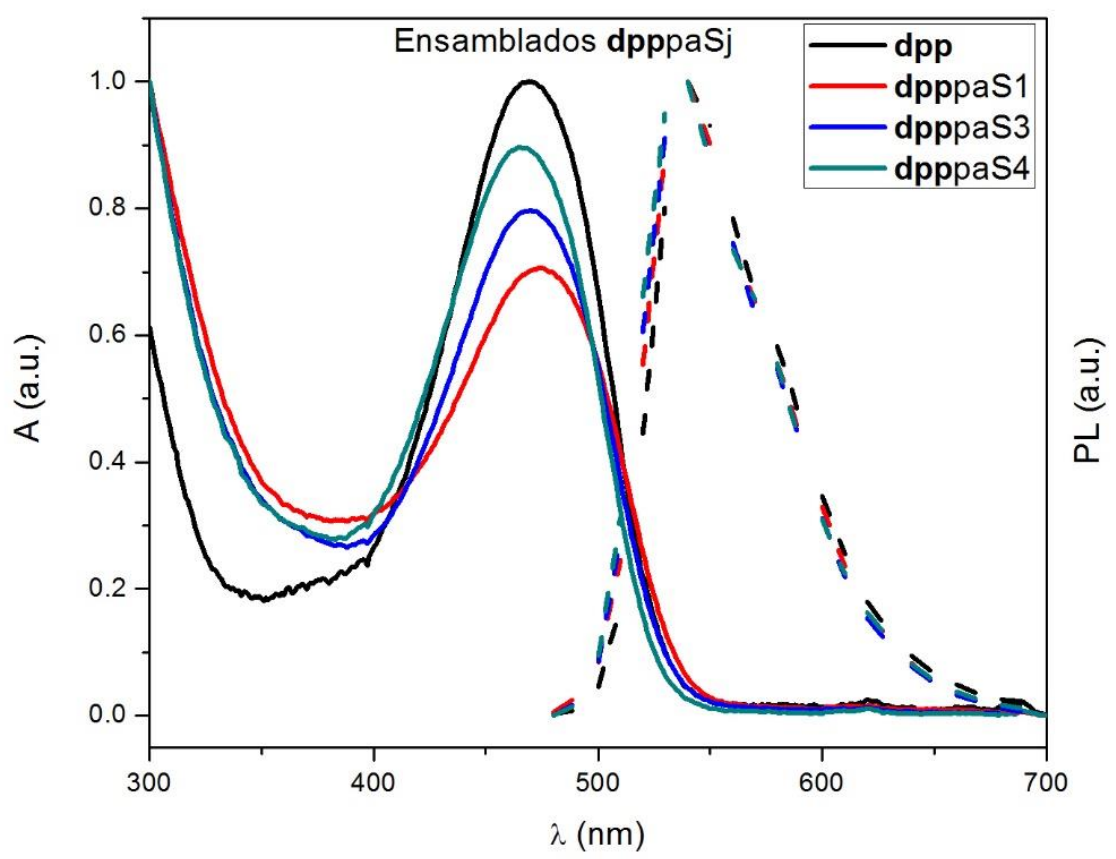

Figura 6.8. Espectros de atenuación (línea continua) y de emisión (línea cortada) normalizados para díadas dpppaSj.

\begin{tabular}{ccc}
\hline Muestra & $\lambda_{\text {uv vis }}(\mathrm{nm})$ & $\lambda_{\mathrm{PL}}(\mathrm{nm})$ \\
\hline dpp & 469 & 540 \\
dpppaS1 & 474 & 540 \\
dpppaS3 & 470 & 540 \\
dpppaS4 & 465 & 540
\end{tabular}

Tabla 6.7. Máximos de absorción y emisión para dpp y las díadas dpppaSj. Se sombrean los valores para el colorante libre como referencia.

La variación de la posición del máximo de atenuación, pero no del máximo de emisión, muestra que la modificación de las superficies de energía potencial para el estado excitado y fundamental NO llega a afectar la distribución de probabilidad de decaimiento por los distintos canales de desactivación del estado excitado, con lo cual la disminución en la intensidad de emisión para $A \sim$ cte se debe a nuevas vías de decaimiento, la cual asignamos a un proceso de transferencia de carga debido a los potenciales redox de los estados involucrados (Capítulo 5 y referencias [13], [27]). Para obtener más información sobre las vías de decaimiento del estado excitado $\mathrm{dpp}^{*}$ en las díadas hicimos medidas de emisión resuelta en el tiempo usando TCSPC, detectando en el máximo de emisión para cada díada y para el colorante libre $\left(\lambda_{\mathrm{ex}}=405 \mathrm{~nm}, \lambda_{\mathrm{em}}=540 \mathrm{~nm}\right)$. A su vez, suponiendo válida la aproximación (6.1) pudimos estimar las constantes de transferencia de carga, que presentamos a continuación junto a los parámetros del ajuste del decaimiento de PL. 


\begin{tabular}{|c|c|c|c|c|c|c|c|}
\hline Muestra & $\Phi_{\mathrm{PL}} / \Phi_{\mathrm{PL}, 0}$ & $\Delta \mathrm{G}^{\circ}(\mathrm{kj} / \mathrm{mol})$ & $T_{\text {dpp }}$ (ns) & $b_{1}$ (a.u.) & $T_{d p p p a s j}(n s)$ & $b_{2}$ (a.u.) & $\mathrm{k}_{\mathrm{eT}}\left(\mathrm{ps}^{-1}\right)$ \\
\hline$d p p$ & 1.0000 & & 9.01 & 5472.8 & & & \\
\hline dpppaS1 & 0.30319 & $-4.31 \mathrm{E}+01$ & 9.01 & 4261.3 & 2.55 & 1293.4 & 0.00028 \\
\hline dpppaS3 & 0.78639 & $-3.89 E+01$ & 9.01 & 4928.2 & 3.11 & 527.38 & 0.00021 \\
\hline dpppaS4 & 0.80975 & $-3.29 \mathrm{E}+01$ & 9.01 & 4689.6 & 3.54 & 750.79 & 0.00017 \\
\hline
\end{tabular}

Tabla 6.8. Parámetros fotoquímicos medidos y estimados para el proceso de PeT en díadas dpppaSj. Se sombrean los valores para el colorante libre como referencia.

Los decaimientos de PL para las díadas pueden ajustarse con una función biexponencial, lo cual sugiere o bien la presencia de dpp libre, lo cual es esperable como se mencionó en la sección 6.3.1., o la existencia de estados que evolucionan dentro de la ventana temporal del experimento. Para elucidar cual de los mecanismos es el que mejor describe los datos experimentales de TCPSC, realizamos experimentos de TAS en una ventana temporal de ca. $5 \mathrm{~ns}$ con resolución de 0.1 ps. Realizamos todas las medidas según se describió en el capítulo 3 manteniendo las mismas condiciones experimentales $\left(\lambda_{\mathrm{ex}}=480 \mathrm{~nm}, 150 \mu \mathrm{j} / \mathrm{pulso}, \mathrm{A}_{480} \sim 0.8\right)$ y usando un supercontinuo de luz de análisis lo más estable posible a lo largo de los experimentos. Las matrices de datos fueron analizadas como se describe en el capítulo 3 usando análisis global y objetivo. Las trazas típicas obtenidas en estos experimentos se muestran a continuación.
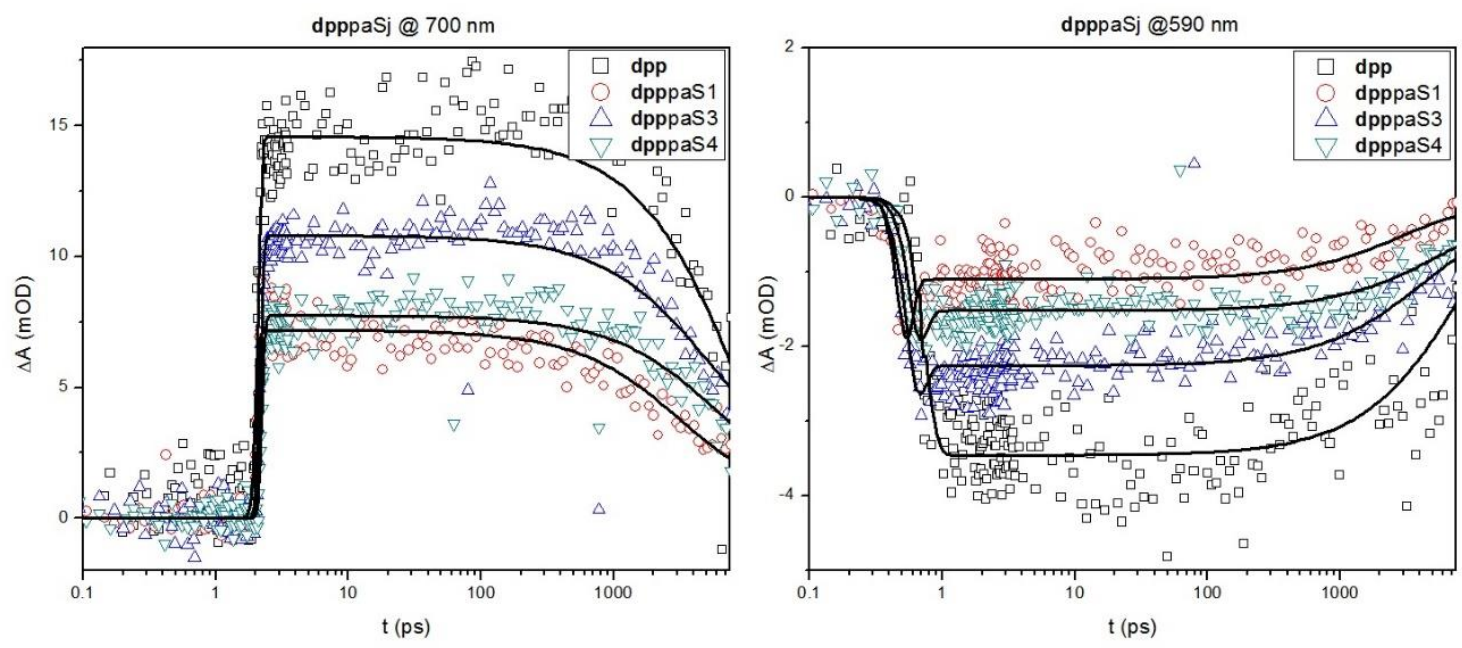

Figura 6.9. Trazas obtenidas en experimentos de TAS para longitudes de onda características del sistema: (a) absorción fotoinducida a $700 \mathrm{~nm}$ y (b) emisión estimulada a $590 \mathrm{~nm}$. En símbolos vacíos se presentan los datos experimentales y en línea contínua los ajustes según el modelo empleado.

Si consideramos solamente un modelo de decaimientos paralelos, el ajuste resulta insuficiente. Por lo tanto, recurrimos a un modelo particular que considera la recombinación de cargas conduciendo tanto al estado singlete excitado ${ }^{1} \mathrm{dpppaSj}\left(\mathrm{k}_{\mathrm{bet}}\right) \mathrm{o}$ a un estado distinto $\left(\mathrm{k}_{\mathrm{cr}}\right)$ que queda por fuera de la ventana temporal. Este modelo es muy similar al propuesto para las díadas usando porfirinas, la gran diferencia es que en el caso de estas díadas el uso de TAS permite la estimación de $k_{\text {bet }}$ y $k_{\text {cr. }}$. Es importante notar que dicha estimación será de importancia o no dependiendo de que $1 / k_{i}$ esté dentro del rango temporal estudiado (cientos de fs hasta pocos ns). El modelo se ejemplifica en la figura 6.10 y los espectros asociados a especies (SAS, del inglés Species Associated Spectra) que resultan del análisis aparecen en la figura 6.11. 


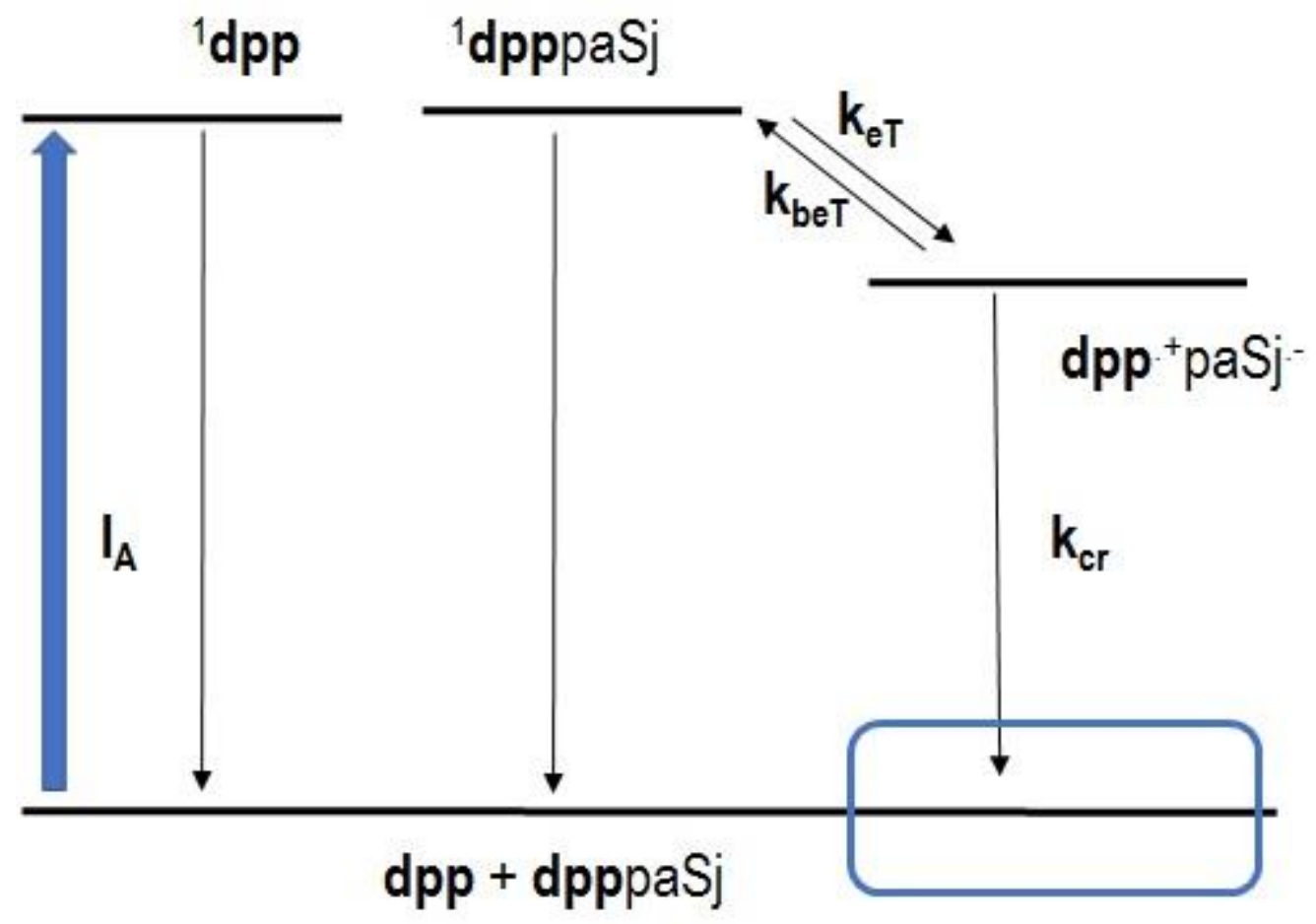

Figura 6.10. Esquema cinético empleado para el análisis de las medidas de TCSPC y TAS en díadas que contienen dpp. El rectángulo celeste representa a los distintos estados de spin lo cuales pueden producirse por recombinación de carga y su posterior relajación.

Los SAS obtenidos luego de analizar los datos de TAS presentan señales claras típicas del colorante empleado, como son la absorción fotoinducida (PIA, del inglés Photo Induced Absorption) a $700 \mathrm{~nm}$ debida a la absorción de 1dpp y la banda de emisión estimulada (SE, del inglés Stimulated Emission) a $600 \mathrm{~nm}$. En el caso de dpp libre en solución, estas 2 señales se identifican claramente y decaen con el mismo tiempo de vida que el determinado por TCSPC. Al considerar las díadas dpppaSj, es necesario hacer un trabajo previo sobre los datos. Para bajar al mínimo la contribución del dpp libre, los datos de las díadas son filtrados con el primer componente de la descomposición en valores singulares (SVD) de la matriz de datos de dpp libre. Este primer componente describe bien el comportamiento espectro-temporal del dpp. Por lo tanto, las señales que aún quedan son asignables puramente a la díada. Sobre estos datos filtrados, se realizó el análisis empleando el modelo ejemplificado en 6.10, considerando 2 compartimentos. En estos casos, uno de los espectros, $\mathrm{SAS}_{1}$, es muy similar al mostrado por ${ }^{1} \mathrm{dpp}$ libre, con lo cual este espectro puede asignarse al estado singlete del colorante unido a la nanopartícula, ${ }^{1} \mathrm{dpppaSj}$ [27]. Por otro lado, el espectro $\mathrm{SAS}_{2}$ presenta una reducción en el ancho a mitad de pico de la señal a $700 \mathrm{~nm}$ con respecto a $S A S_{1}$, que puede entenderse como la termalización de ${ }^{1} \mathrm{dpppaSj}$, y una señal (posiblemente PIA) a 585 - $590 \mathrm{~nm}$ montada sobre la banda de SE. Esta señal es muy similar a la obtenida para el estado excitado PA-BU' Si nps al nivel de la banda de conducción (CB). En bibliografía se ha reportado que para QDs de distinta naturaleza (CdSe, CdTe, Si) una vez poblado el estado excitado, ya sea por excitación directa por un proceso de absorción o por transferencia electrónica, el espectro observado por TAS es muy similar [43]-[45]. Teniendo en cuenta que los experimentos se realizaron excitando a $480 \mathrm{~nm}$, podemos descartar la excitación directa de las Si nps en las díadas. A su vez, si efectivamente estuviésemos generando el estado dpppaSj* este se manifestaría a tiempos cortos (i.e. cientos de fs), fuera del intervalo alcanzado en estos experimentos. Por estos motivos, asignamos el espectro $\mathrm{SAS}_{2}$ a dpp ${ }^{+} \mathrm{Sj}-$ - Para estar completamente seguros de esta asignación deberíamos encontrar señales del catión radical, que en este caso particular presenta señales fuera de la ventana espectral utilizada (normalmente los cationes radicales del dpp muestran señales en el NIR) [27]. 

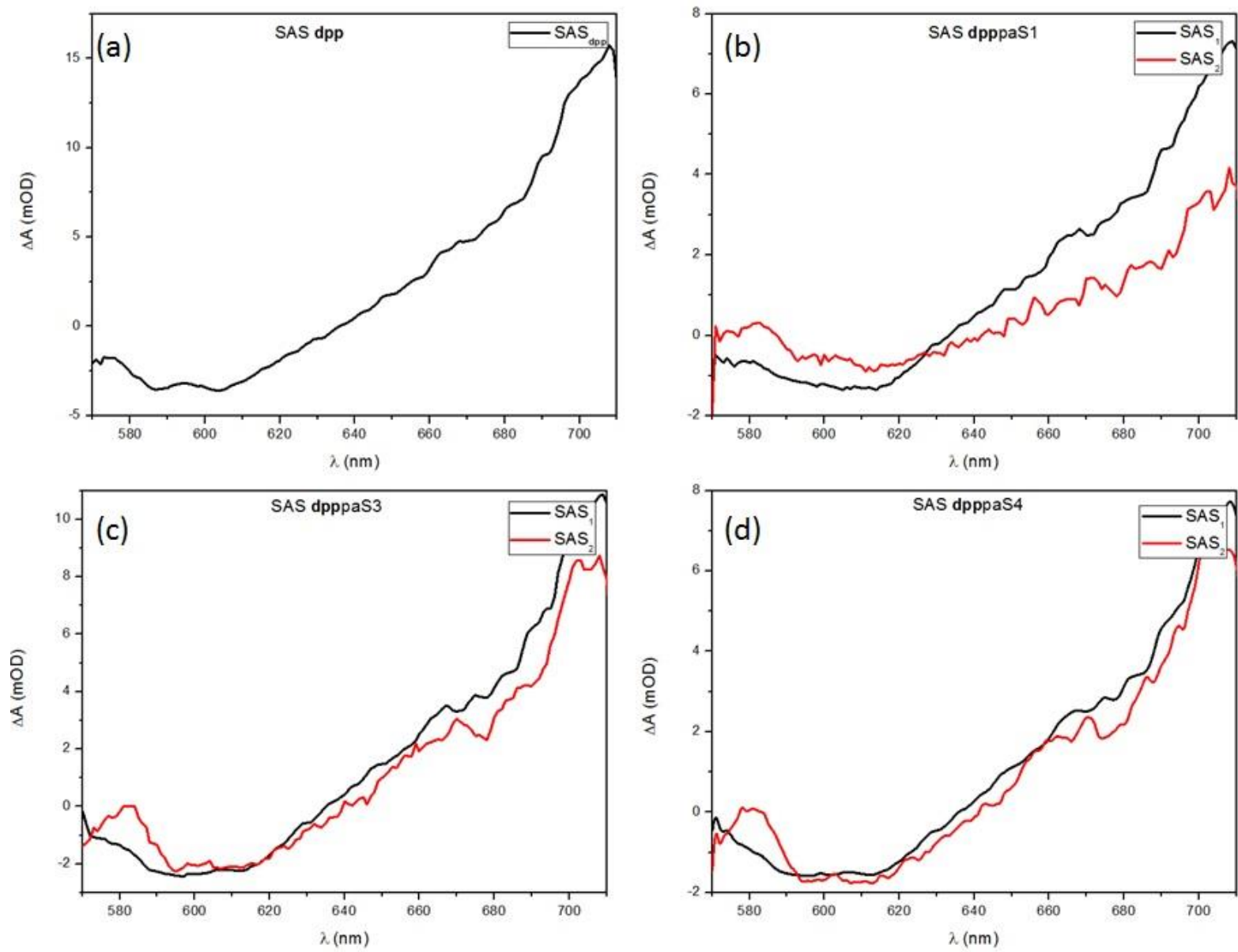

Figura 6.11. SAS obtenidos al analizar los datos de TAS para (a) el colorante dpp libre y las díadas (b) dpppaS1, (c) dpppaS3 y (d) dpppaS4.

Teniendo en cuenta el modelo empleado, las constantes obtenidas se listan en la siguiente tabla.

\begin{tabular}{ccccccc}
\hline Díada & $\boldsymbol{\Delta} \mathbf{G}^{\circ}(\mathbf{k j} / \mathbf{m o l})$ & TD $(\mathbf{p s})$ & $\mathbf{k}_{\mathrm{eT}}\left(\mathbf{p s}^{-1}\right)$ & $\boldsymbol{\Phi}_{\mathrm{eT}}$ & $\mathbf{k}_{\text {beT }}\left(\mathbf{p s}^{-1}\right)^{*}$ & $\mathbf{k c r}^{\prime}\left(\mathbf{p s} \mathbf{- 1}^{* *}\right.$ \\
\hline dpppaS1 & $-4.31 \mathrm{E}+01$ & 2547.9 & 0.00028 & 0.71 & $1.00 \mathrm{E}-06$ & $5.00 \mathrm{E}-05$ \\
dpppaS3 & $-3.89 \mathrm{E}+01$ & 3114.4 & 0.00021 & 0.65 & $3.00 \mathrm{E}-06$ & $5.00 \mathrm{E}-05$ \\
dpppaS4 & $-3.29 \mathrm{E}+01$ & 3539 & 0.00017 & 0.60 & $5.00 \mathrm{E}-06$ & $5.00 \mathrm{E}-05$
\end{tabular}

Tabla 6.8. Parámetros fotoquímicos correspondientes al proceso de PeT en díadas dpppaSj.( $\left.{ }^{*}\right)$ La variación de kbeT muestra la diferencia en la energética de cada díada, pero solo puede utilizarse como guía y no como un dato confiable. $\left(^{* *}\right)$ En el caso de kcr, toma un valor arbitrario que está fuera de la escala temporal sondeada.

Por un lado, vemos que para el proceso de $\mathrm{PeT}, \mathrm{k}_{\mathrm{e}}$ у у $\Phi_{\mathrm{eT}}$ aumentan con $\Delta \mathrm{G}^{0}$. Como dijimos previamente, al finalizar el capítulo veremos el efecto de $\Delta G^{\circ}$ sobre keT para sistemas con el mismo aceptor, para evitar interferencias debidas a efectos de la superficie. Por el otro, los valores obtenidos para kbeT y kcr están fuera del intervalo temporal sondeado, con lo cual solo deben considerarse como guías al momento de analizar el sistema y no como bases para cálculos posteriores. 
De todos modos, es interesante notar que el estado de cargas separadas sobrevive a la escala temporal estudiada y es generado excitando en la región visible del espectro EM, lo que abre las puertas a aplicaciones en fotoelectrodos de gran área superficial, útiles en la generación de combustibles a partir de energía solar.

\subsubsection{Díadas empleando Carotenoides: CkpaSj.}

Por último, sintetizamos díadas usando como colorantes a carotenoides, que presentan potenciales de oxidación en el estado excitado lo suficientemente altos, ya sea para garantizar la transferencia de carga 0 , caso contrario, explorar la región invertida de Marcus para reacciones de transferencia de carga. Estas díadas fueron estudiadas mayormente por espectroscopía de absorción UV-Vis estacionaria y resuelta en el tiempo. Por otro lado, este sistema resultó ser el más complejo para manipular, debido a la reactividad de los carotenoides frente a oxígeno y a la luz. Los espectros de absorción UV-vis de los carotenoides usados se presentan en la figura 6.12 .

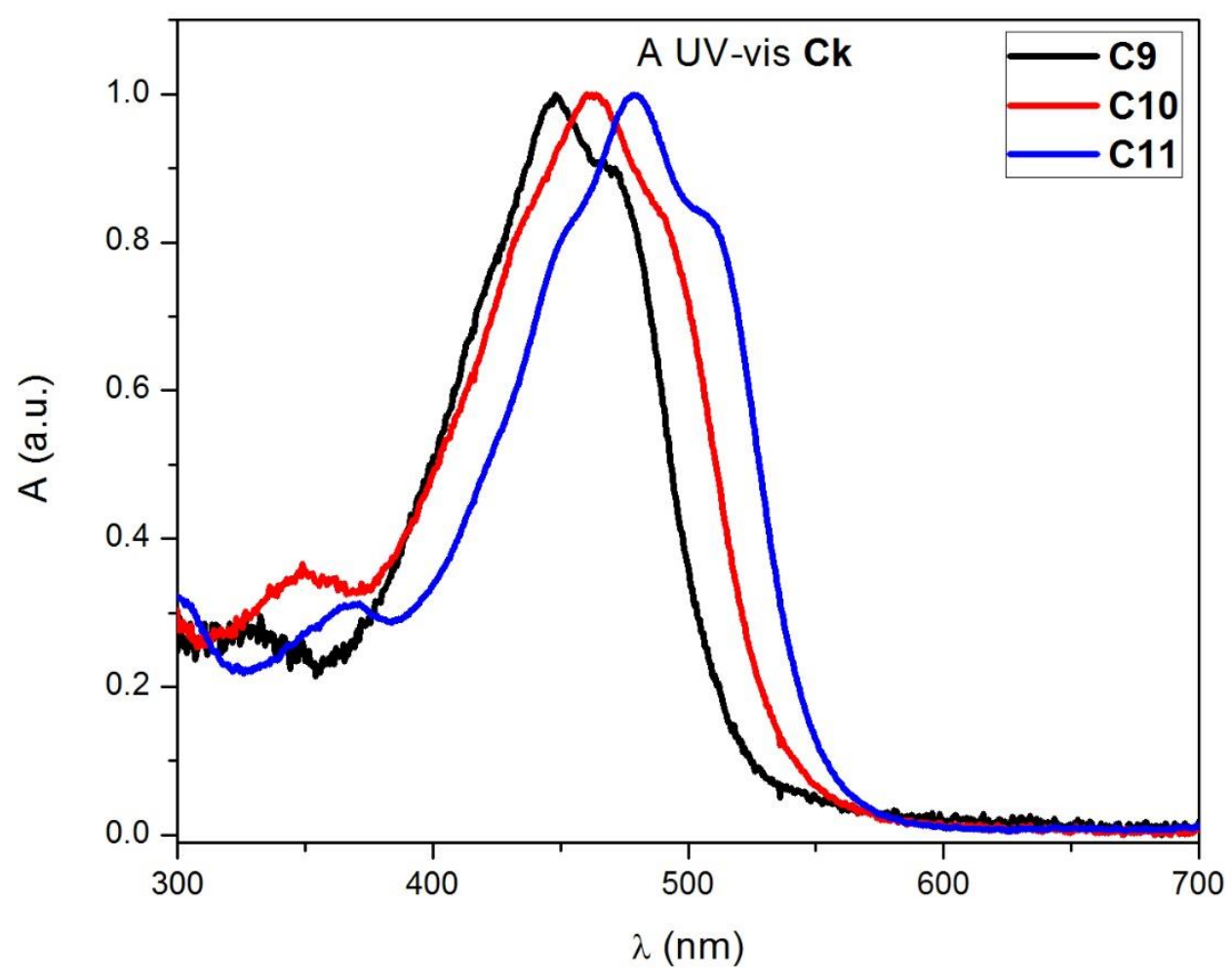

Figura 6.12. Espectros de absorción UV-vis de los carotenoides empleados.

A diferencia de los colorantes usados anteriormente, en este caso nos enfocaremos en primer lugar en comparar el comportamiento de los 3 carotenoides con el mismo aceptor. En línea con esta idea, presentamos los espectros de atenuación UV-vis y de emisión estacionarios para las díadas que usan como aceptor S1 (CkpaS1) y S3 (CkpaS3). 

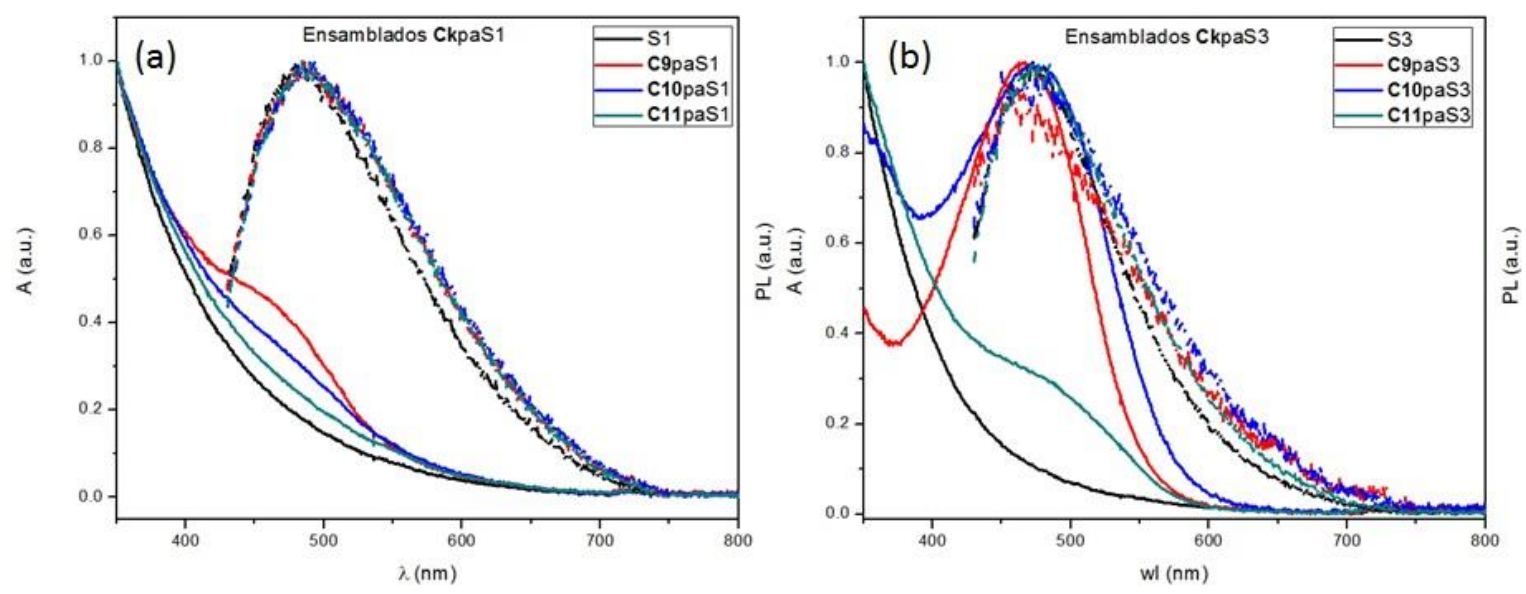

Figura 6.13. Espectros de atenuación (línea continua) y de emisión (línea cortada) normalizados para díadas (a) CkpaS1y (b) CkpaS3.

En la figura se puede ver la banda de los carotenoides superpuesta con la absorción de las PA-BU' Si nps. También se presenta en el mismo gráfico el espectro de emisión de las díadas excitando ( $\lambda$ ex= $350 \mathrm{~nm}$ ) en la región donde domina la absorción por parte de las Si nps. La intensidad de PL disminuye en presencia de carotenoides, sin embargo no proseguimos estudiando ese efecto, ya que el fin de utilizar colorantes auxiliares es excitar las díadas en el visible y activar procesos fotoinducidos prescindiendo de la región UV del espectro EM. Los máximos de absorción y emisión para las díadas CkpaS1 y CkpaS3 aparecen en la tabla 6.9.

\begin{tabular}{ccc}
\hline Muestra & $\lambda_{\text {uv vis }}(\mathrm{nm})$ & $\lambda_{\mathrm{PL}}(\mathrm{nm})$ \\
\hline C9 & 449 & \\
C9paS1 & 465 & 485 \\
C10 & 465 & 462 \\
C10paS1 & 465 & \\
C10paS3 & 472 & 487 \\
C11 & 479 & 483 \\
C11paS1 & 440 & \\
C11paS3 & 480 & 484 \\
\hline
\end{tabular}

Tabla 6.9. Máximos de absorción y emisión para los carotenoides $\mathbf{C} 9, \mathbf{C} 10, \mathbf{C} 11$ y las díadas que estos constituyen. Se sombrean los valores para el colorante libre como referencia.

El cambio en la posición de los máximos de absorción y el ensanchamiento de los picos nos muestra que la superficie se las PA-BU' Si nps modifica la estructura electrónica de los carotenoides empleados. Para estudiar el efecto de esta perturbación sobre la fotoquímica de los colorantes hicimos experimentos de TAS. Las condiciones experimentales fueron las mismas para todas las determinaciones ( $\lambda_{\mathrm{ex}}=480 \mathrm{~nm}, 50 \mathrm{uj} / \mathrm{pulso}, \mathrm{A}_{480}$ $\sim 0.5$ ), y para evitar la fotodegradación de los carotenoides se empleó una cubeta rotante.

Si bien los carotenoides presentan una compleja estructura de estados excitados [17], [30][46] su fotoquímica se estudia desde hace mucho tiempo y está muy bien documentada, aunque existe mucha controversia sobre 
la naturaleza de algunos estados excitados. Los carotenoides libres presentan PIAs claras y estrechas, asignadas en bibliografía a transiciones $S_{1} \rightarrow S_{N}$, con $S_{1}$ mostrando distintos estados excitados vibracionales [17]-[19], [46]-[48][49]. A su vez, la posición de la banda de PIA depende del largo y la conformación de la cadena insaturada del carotenoide. En el caso particular de carotenoides que presenten grupos carbonilos, las bandas se ensanchan y presentan variaciones con la polaridad del solvente [19], [50][51].

El acoplamiento de los carotenoides a PA-BU Si nps cambia sustancialmente el comportamiento fotofísico. En general, se observan bandas más anchas y menos resueltas, lo cual podría indicar la participación de varios estados en el proceso de relajación. En bibliografía se encuentran estudios que involucran carotenoides en díadas o sistemas más complejos que presentan comportamientos similares [30][17], [52].

En primer lugar, analizamos el comportamiento de $\mathbf{C 9}$ y la díada C9paS1. No pudimos estudiar la díada C9paS3 debido a que el carotenoide se degrado en el tiempo de almacenado. El comportamiento de $\mathbf{C 9}$ es similar al observado para distintos carotenoides con 9 dobles enlaces [18], [53]. En la figura 6.12 presentamos algunas trazas que muestran la evolución de los estados excitados generados en el experimento.

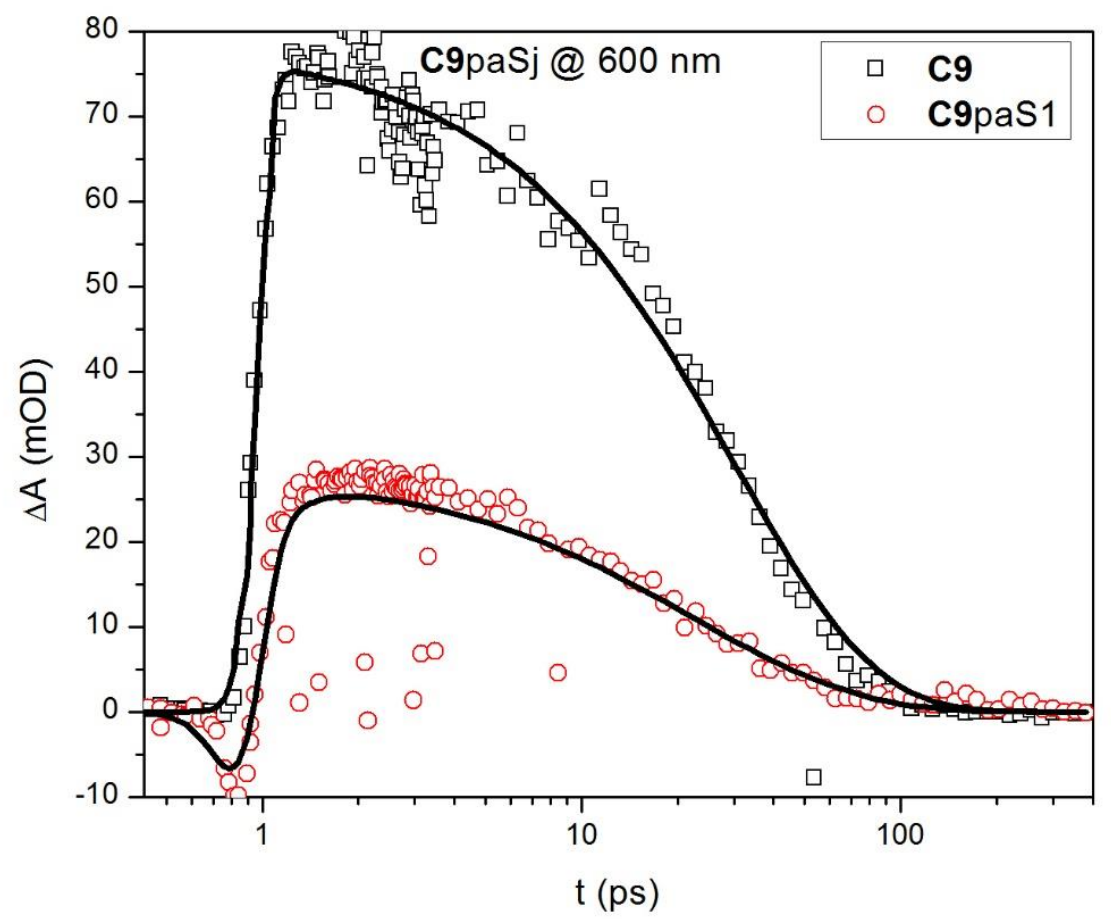

Figura 6.12. Trazas obtenidas en experimentos de TAS para el proceso de absorción fotoinducida a $600 \mathrm{~nm}$. En símbolos vacíos se presentan los datos experimentales y en línea contínua los ajustes según el modelo empleado.

Para explicar los datos obtenidos por TAS es necesario considerar 2 compartimentos que decaen de forma secuencial $(1 \rightarrow 2 \rightarrow 3)$ con constantes de velocidad $k_{1}, k_{2}$ y $k_{3}$ respectivamente. Este modelo se muestra en la figura 6.13 . 


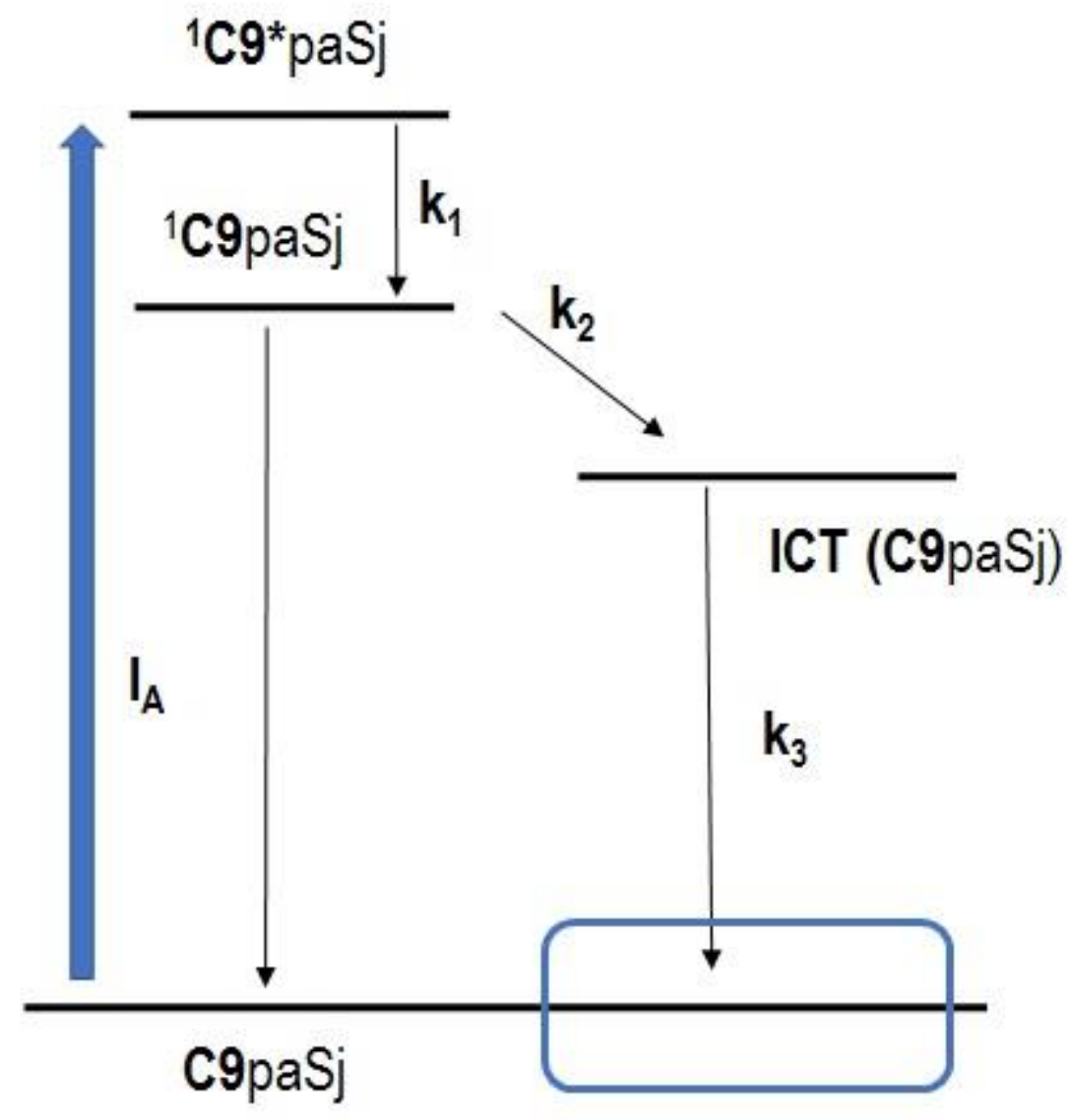

Figura 6.13. Esquema cinético empleado para el análisis de las medidas de TAS en diadas que contienen C9, C10 y C11. El rectángulo celeste representa a los distintos estados de spin lo cuales pueden producirse por recombinación de carga y su posterior relajación

Los espectros asociados (EAS, del inglés Evolving associated Spectra) muestran máximos alrededor de 600 $\mathrm{nm}$. El primer EAS presenta un máximo en ca. $605 \mathrm{~nm}$, y una banda más pequeña en $695 \mathrm{~nm}$. Sabiendo que el tiempo de vida del estado excitado generado por el proceso de absorción $\left({ }^{2} \mathbf{C} 9\right)$ y que su tiempo de vida es de ca. $50 \mathrm{fs}$, podemos suponer que dicho estado no es detectable en nuestra configuración experimental (IRF de aproximadamente $120 \mathrm{fs}$ ). A su vez, teniendo en cuenta la constante de velocidad para el decaimiento de este primer EAS $\left(\mathrm{k}_{1} \sim 5.1 \mathrm{ps}^{-1}\right)$ y las señales que presenta, lo asignamos al estado vibracionalmente excitado ${ }^{*} S_{1}\left({ }^{1} \mathbf{C} 9^{*}\right)$ generado por relajación de ${ }^{2} \mathbf{C} 9$. ${ }^{1} \mathbf{C} 9^{*}$ decae por conversión interna a $S_{1}\left({ }^{1} \mathbf{C} 9\right)$, que no puede poblarse por absorción directa desde $S_{0}$. En línea con esta afirmación, EAS 2 se asignó a ${ }^{1} \mathbf{C} 9$, cuyo tiempo de vida es $1 / k_{2}=30.3$ ps. Estas asignaciones están de acuerdo con lo reportado en literatura. 

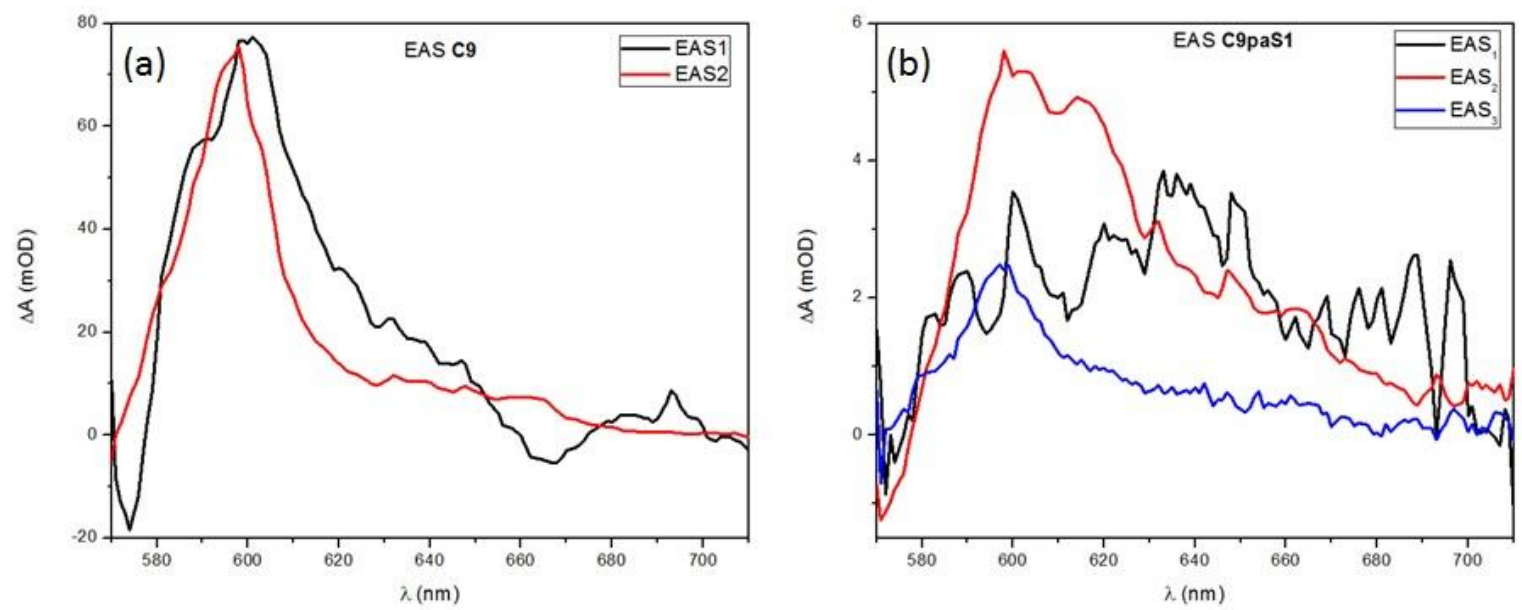

Figura 6.14. SAS obtenidos al analizar los datos de TAS para (a) el colorante C9 libre y la díada (b) C9paS1.

Por otro lado, para C9paS1 observamos un primer EAS un tanto ruidoso, que decae en 285 fs que puede asignarse al análogo a ${ }^{1} \mathbf{C 9}{ }^{*}$ para la díada, ${ }^{1} \mathbf{C} \mathbf{9}^{*} \mathrm{paS} 1$. Si bien presenta un alto nivel de ruido, la constante de velocidad y el espectro no condicen con el correspondiente al artefacto coherente ni a ruido espectral, el cual es considerado aparte en el análisis (ver la sección 2.8.2 del capítulo 2). Este relaja a ${ }^{1} \mathbf{C} 9\left(\mathrm{EAS}_{2}\right)$ por conversión interna y que luego evoluciona a un estado de transferencia de carga (nombrado ICT, del inglés Intermolecular Charge Transfer), el cual se manifiesta en carotenoides con residuos que se pueden comportar como aceptores de carga [51]. Luego este estado relaja en 200 ps. En la figura 6.14 mostramos los EAS obtenidos por análisis global.

En el caso de $\mathbf{C 1 0}$ y $\mathbf{C 1 1}$, ocurren procesos similares a los descriptos para $\mathbf{C 9}$. En el caso de las díadas, los mayores cambios se observan al modificar las nanopartículas que se comportan como aceptores de carga, para formar el estado ICT. Asumimos que la formación de ICT es competitiva con la relajación al estado fundamental desde $1 \mathrm{CkpaSj}$, con lo cual propusimos considerar:

$$
\mathrm{k}_{3, \mathrm{CkpaSj}}=\mathrm{k}_{3, \mathrm{Ck}}+\mathrm{k}_{\mathrm{ICT}, \mathrm{j}}
$$

En todas las díadas, el uso de Si nps con menores potenciales de reducción lleva a una menor expresión de la banda del estado ICT. Este detalle particular, queda más claro al analizar las constantes de velocidad para la formación ICT (EAS 3 ), las cuales disminuyen al aumentar la diferencia de energía libre para el proceso de PeT (tabla 6.4). 

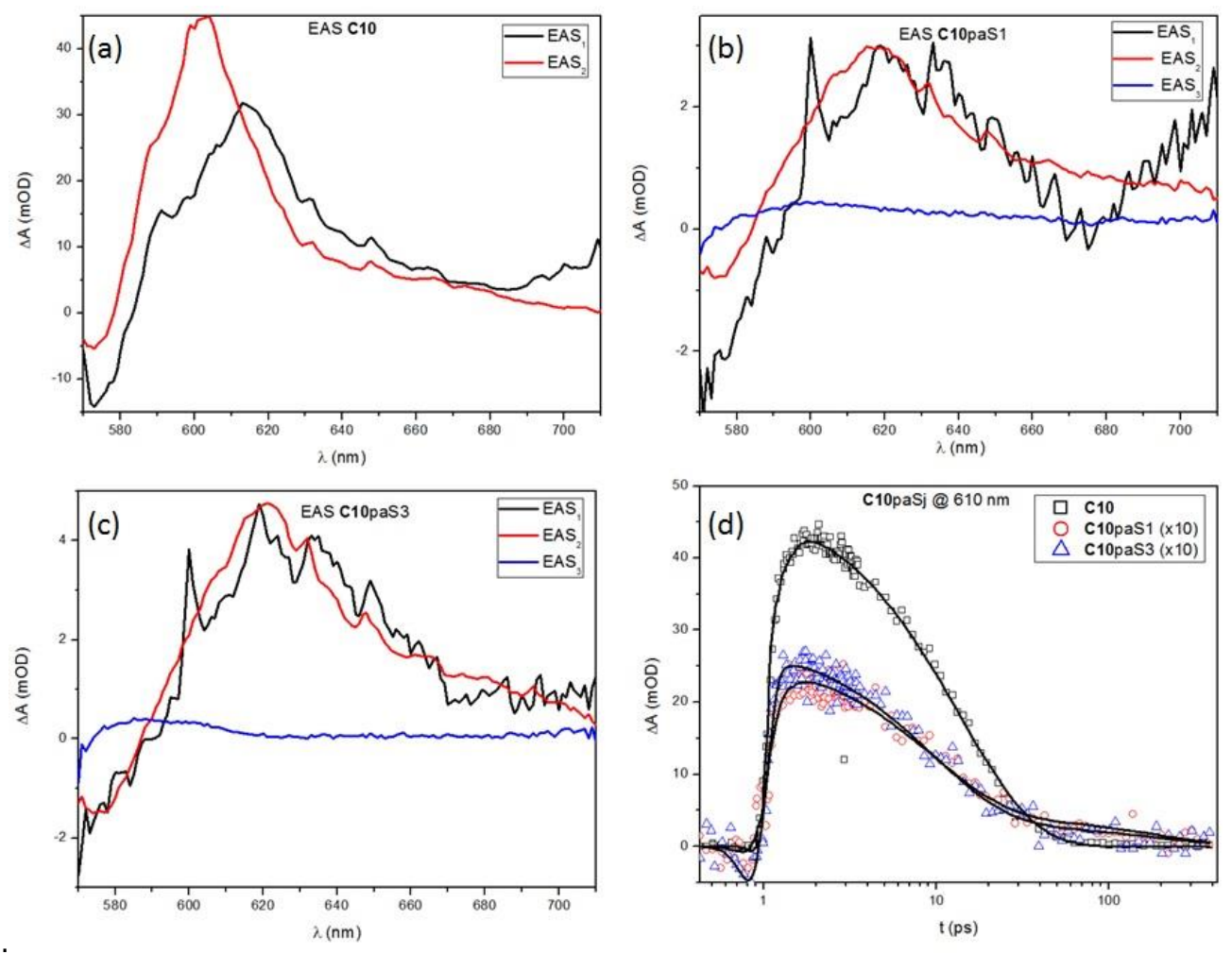

Figura 6.15. SAS obtenidos al analizar los datos de TAS para (a) el colorante C10 libre y las díadas (b) C10paS1 y (c) C10paS3. En (d) se presentan las trazas obtenidas por TAS para la absorción fotoinducida a $610 \mathrm{~nm}$.
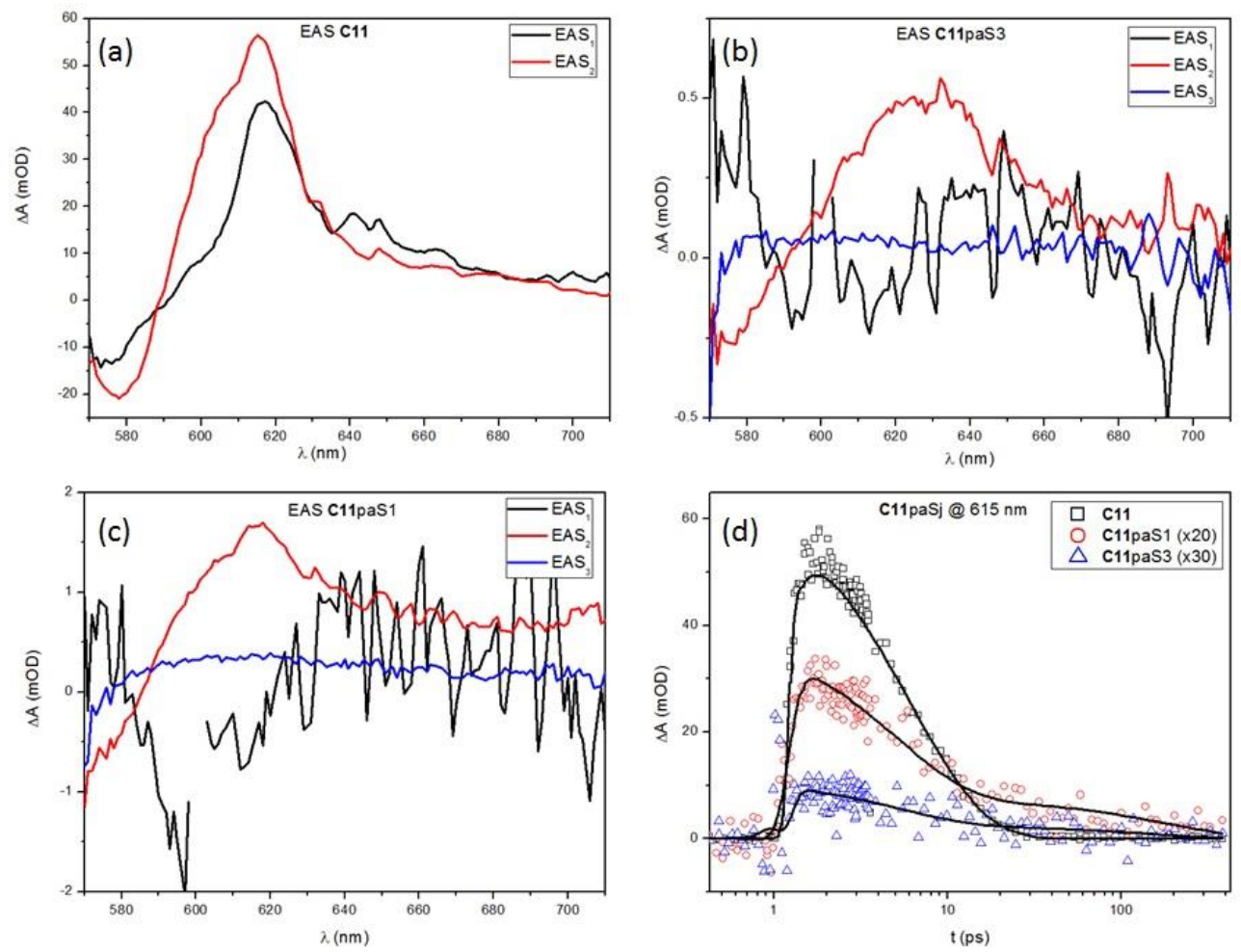

Figura 6.15. SAS obtenidos al analizar los datos de TAS para (a) el colorante C11 libre y las díadas (b) C11paS1 y (c) C11paS3. En (d) se presentan las trazas obtenidas por TAS para la absorción fotoinducida a $615 \mathrm{~nm}$. 


\begin{tabular}{cccccc}
\hline Muestra & $\Delta \mathbf{G}^{\mathbf{0}}(\mathbf{k j} / \mathbf{m o l})$ & $\mathbf{k}_{1}\left(\mathbf{p s}^{-1}\right)$ & $\mathbf{k}_{2}\left(\mathbf{p s}^{-1}\right)$ & $\mathbf{k}_{\text {ICT }}\left(\mathbf{p s}^{-1}\right)$ & $\mathbf{k}_{3}\left(\mathbf{p s}^{-1}\right)$ \\
\hline C9 & & 5.10 & 0.033 & & \\
C9paS1 & -49.53 & 3.53 & 0.047 & 0.014 & 0.030 \\
C9paS3 & -45.33 & & & & \\
C10 & & 3.85 & 0.073 & & \\
C10paS1 & -36.09 & 3.85 & 0.10 & 0.031 & 0.0052 \\
C10paS3 & -31.89 & 3.85 & 0.099 & 0.026 & 0.0055 \\
C11 & & 3.48 & 0.16 & & \\
C11paS1 & -29.78 & 4.44 & 0.20 & 0.035 & 0.0052 \\
C11paS3 & -25.58 & 4.68 & 0.19 & 0.028 & 0.0070
\end{tabular}

Tabla 6.10. Constantes de velocidad para los procesos fotoquímicos en los carotenoides $\mathbf{C 9}, \mathbf{C} 10$ y $\mathbf{C 1 1}$ libres y en las díadas $\mathbf{C 9 p a S j}$, C10paSj y C11paSj estudiadas. Se sombrean los valores para el colorante libre como referencia.

Los procesos estudiados en las díadas sintetizadas pueden resumirse en la siguiente figura.

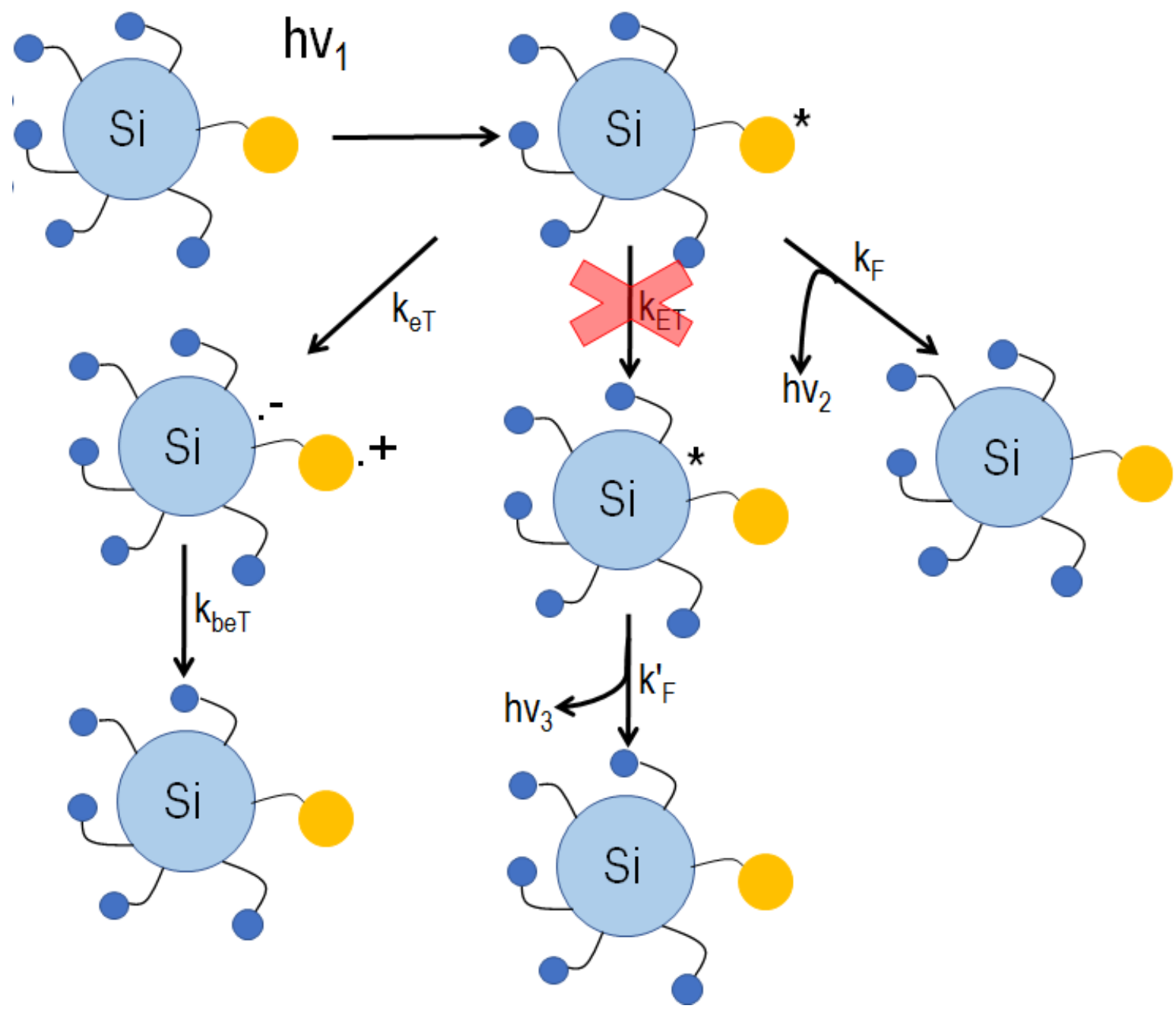

Figura 6.16. Procesos fotoquímicos factibles en los sistemas sintetizados. La vía de transferencia de energía es poco probable, ya que las bandas de emisión de los cromóforos empleados no se superponen con la absorción de las Si nps. 
Finalmente, al analizar todas las díadas estudiadas puede observarse que, si consideramos díadas con el mismo aceptor (a fin de analizar sistemas donde el efecto de la superficie sea al menos similar) vemos el siguiente comportamiento.

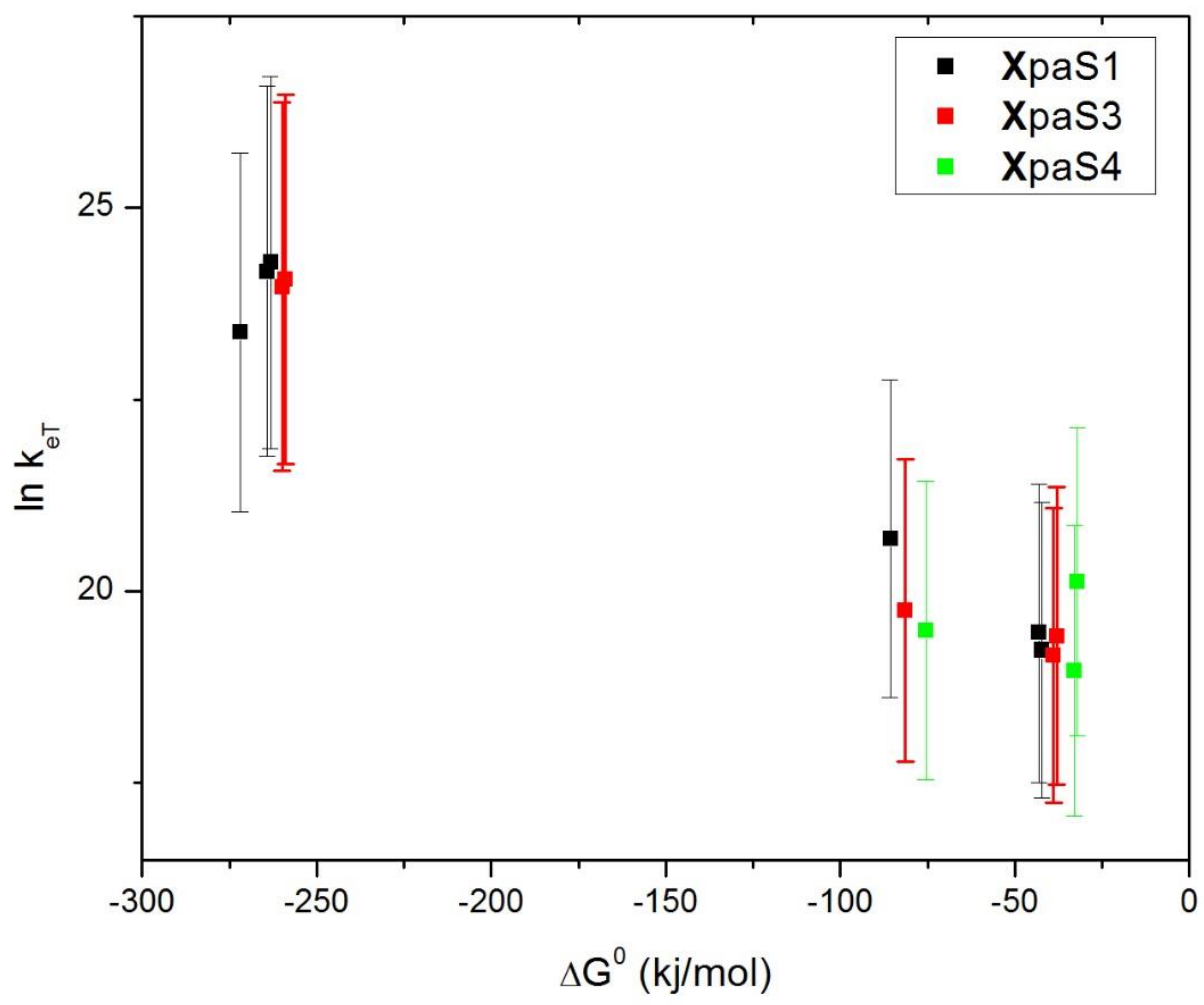

Figura 6.16. Variación de las constantes de transferencia de carga $\left(k_{e}\right)$ en función de la variación de energía libre para el proceso de separación de cargas fotoinducido $\left(\Delta \mathrm{G}^{\circ}\right)$.

Podemos ver que, se diferencian claramente 2 regiones: por debajo encima de $\sim-100 \mathrm{~kJ} / \mathrm{mol}$ las constantes de velocidad aumentan con $\Delta G^{\circ}$, mientras que por debajo de $\sim-240 \mathrm{kj} / \mathrm{mol}$ disminuyen. Este comportamiento puede explicarse en el marco de la teoría de Marcus, en el cual si bien la reacción es termodinámicamente muy favorable implica un reordenamiento del entorno dieléctrico muy importante, lo cual restringe los valores de keT. Este tipo de comportamiento se encuentra documentado en bibliografía para diversas díadas sintéticas[1], [54] e incluso en sistemas nanoestructurados [55][53], [56], [57].

\subsection{Conclusiones}

Se sintetizaron con éxito diversas díadas que contienen cromóforos orgánicos junto a Si nps. Estas díadas fueron sometidas a un proceso de purificación lo más exhaustivo posible, para evitar la interferencia de comóforos libres, sin embargo esta tarea fue muy difícil de llevar adelante. Se eligió el método de purificación que mejores resultados dio, pero que aún dista de ser el óptimo.

Las propiedades ópticas de estos ensamblados fueron estudiadas en detalle usando una batería de técnicas espectroscópicas las cuales permitieron obtener información útil para esclarecer la dinámica de los estados excitados involucrados. 
Los procesos de transferencia de carga fueron interpretados en términos de la teoría de Marcus, observando que en general el proceso de separación de cargas se encontraba en la región normal, salvo en el caso de díadas conteniendo carotenoides que se encuentran en la región inversa.

Por último, a través de los estudios realizados no podemos concluir nada sobre la naturaleza del estado al cual relaja el estado de carga separada formado. Este proceso involucra probablemente la recombinación del par, pudiendo conducir a productos de distintos estados de spin. Para obtener mayor información, deberían realizarse estudios de TAS en los $\mu$ s o de EPR (Electron Paramagnetic Resonance) que quedaron por fuera de esta tesis.

Los resultados de este capítulo constituyen una base fotoquímica para la aplicación de estas díadas en aplicaciones en fotoelectrodos de gran área superficial, útiles en conversión de energía solar y en celdas de combustible fotoelectroquímicas.

\subsection{Referencias}

[1] D. Gust, T. A. Moore, and A. L. Moore, "Mimicking photosynthetic solar energy transduction," Acc. Chem. Res., vol. 34, no. 1, pp. 40-48, 2001.

[2] P. V. Kamat, "Meeting the clean energy demand: Nanoestructure Architectures for Solar Energy Conversion," Phys. Chem., vol. 392, pp. 2834-2860, 2007.

[3] D. Neiner, H. W. Chiu, and S. M. Kauzlarich, "Low-temperature solution route to macroscopic amounts of hydrogen terminated silicon nanoparticles," J. Am. Chem. Soc., vol. 128, pp. 11016-11017, 2006.

[4] R. K. Baldwin, K. a. Pettigrew, J. C. Garno, P. P. Power, G. Y. Liu, and S. M. Kauzlarich, "Room temperature solution synthesis of alkyl-capped tetrahedral shaped silicon nanocrystals," J. Am. Chem. Soc., vol. 124, pp. 1150-1151, 2002.

[5] K. Pettigrew and Q. Liu, "Solution synthesis of alkyl-and alkyl/alkoxy-capped silicon nanoparticles via oxidation of Mg2Si," Chem. ..., no. 31, pp. 4005-4011, 2003.

[6] G. P. Redei, Gel Filtration. 2004.

[7] R. Giovannetti, "The Use of Spectrophotometry UV-Vis for the Study of Porphyrins."

[8] A. Fermi, M. Locritani, G. Di Carlo, M. Pizzotti, S. Caramori, Y. Yu, B. A. Korgel, G. Bergamini, and P. Ceroni, "Light-harvesting antennae based on photoactive silicon nanocrystals functionalized with porphyrin chromophores.," Faraday Discuss., vol. 185, pp. 481-95, 2015.

[9] W. Auwärter, D. Écija, F. Klappenberger, and J. V Barth, "Porphyrins at interfaces," Nat. Publ. Gr., vol. 7, no. 2, pp. 105-120, 2015.

[10] A. Gulino, S. Giuffrida, P. Mineo, M. Purrazzo, E. Scamporrino, G. Ventimiglia, M. E. Van Der Boom, I. Fragala, and I. N. S. T. M. U. Catania, "Photoluminescence of a Covalent Assembled Porphyrin-Based Monolayer : Optical Behavior in the Presence of O 2," pp. 16781-16786, 2006.

[11] J. Schneider, F. Kollho, T. Schindler, S. Bichlmaier, J. Bernardi, T. Unruh, J. Libuda, T. Berger, and O. Diwald, "Adsorption, Ordering, and Metalation of Porphyrins on MgO Nanocube Surfaces: The Directional Role of Carboxylic Anchoring Groups," 2016.

[12] C. A. G. N. Montalbetti and V. Falque, "Amide bond formation and peptide coupling," Tetrahedron, vol. 61, no. 46. pp. 10827-10852, 2005. 
[13] V. I. Vullev and G. Jones, "Photoinduced charge transfer in helical polypeptides," Res. Chem. Intermed., vol. 28, no. 7-9, pp. 795-815, 2002.

[14] M. Gervaldo, P. a Liddell, G. Kodis, B. J. Brennan, C. R. Johnson, J. W. Bridgewater, A. L. Moore, T. a Moore, and D. Gust, "A photo- and electrochemically-active porphyrin-fullerene dyad electropolymer.," Photochem. Photobiol. Sci., vol. 9, no. 7, pp. 890-900, 2010.

[15] J. Li, A. Ambroise, S. I. Yang, J. R. Diers, J. Seth, C. R. Wack, D. F. Bocian, D. Holten, and J. S. Lindsey, "Template-directed synthesis, excited-state photodynamics, and electronic communication in a hexameric wheel of porphyrins," J. Am. Chem. Soc., vol. 121, no. 38, pp. 8927-8940, 1999.

[16] R. W. Wagner, J. Seth, S. I. Yang, D. Kim, D. F. Bocian, D. Holten, and J. S. Lindsey, "Synthesis and Excited-State Photodynamics of a Molecular Square Containing Four Mutually Coplanar Porphyrins," J. Org. Chem., vol. 63, no. 15, pp. 5042-5049, 1998.

[17] T. Polívka and V. Sundström, "Dark excited states of carotenoids : Consensus and controversy," Chem. Phys. Lett., vol. 477, no. 1-3, pp. 1-11, 2009.

[18] D. M. Niedzwiedzki, D. J. Sandberg, H. Cong, M. N. Sandberg, G. N. Gibson, R. R. Birge, and H. A. Frank, "Ultrafast time-resolved absorption spectroscopy of geometric isomers of carotenoids," Chem. Phys., vol. 357, no. 1-3, pp. 4-16, 2009.

[19] D. Zigmantas, V. Sundstr, and H. Helena, "Dynamics of vibrational relaxation in the S 1 state of carotenoids having 11 conjugated C @ C bonds I vka," vol. 355, no. April, pp. 465-470, 2002.

[20] M. Vala, M. Weiter, J. Vy, P. Toman, S. Lu, Z. Qiao, Y. Xu, S. Lin, J. Peng, D. Cao, S. Qu, W. Wu, J. Hua, C. Kong, Y. Long, and H. Tian, “ChemComm," vol. 160, pp. 1181-1186, 2010.

[21] J. J. Romero, M. J. Llansola-portole, D. Arciprete, B. Rodr, A. L. Moore, and C. Gonzalez, "Photoluminescent 1 - 2 nm Sized Silicon Nanoparticles: A Surface- Dependent System," Chem. Mater., 2013.

[22] C. R. Lillo, J. J. Romero, M. L. Portolés, R. P. Diez, and P. C. Caregnato, "Organic-coating of 1-2 nm size silicon nanoparticles : effect on the particle properties," 2015.

[23] P. Caregnato, M. L. Dell'Arciprete, M. C. Gonzalez, C. R. Lillo, H. B. Rodríguez, and J. J. Romero, "Versatile silicon nanoparticles with potential uses as photoluminiscent sensors and photosensitizers," Photochemistry, vol. 44, pp. 322-345, 2017.

[24] S. Farid, J. P. Dinnocenzo, P. B. Merkel, R. H. Young, D. Shukla, and G. Guirado, "Reexamination of the Rehm À Weller Data Set Reveals Electron Transfer Quenching That Follows a Sandros À Boltzmann Dependence on Free Energy," vol. 0, no. Figure 1, pp. 11580-11587, 2011.

[25] B. Valeur, Molecular Fluorescence: Principles and applications, vol. 8. 2001.

[26] J. R. Lakowicz, Principles of fluorescence spectroscopy. 2006.

[27] A. Purc, E. M. Espinoza, R. Nazir, J. J. Romero, K. Skonieczny, A. Jeżewski, J. M. Larsen, D. T. Gryko, and V. I. Vullev, "Gating that Suppresses Charge Recombination - the Role of Mono-NArylated Diketopyrrolopyrrole," J. Am. Chem. Soc., p. jacs.6b04974, 2016.

[28] A. Antoniuk-pablant, G. Kodis, A. L. Moore, T. A. Moore, and D. Gust, "Supporting Information Photoinduced Electron and Energy Transfer in a Molecular Triad Featuring a Fullerene Redox Mediator Antaeres Antoniuk-Pablant, Gerdenis Kodis, Ana L. Moore, ${ }^{*}$ Thomas A. Moore, ${ }^{*}$ and Devens 
Gust*," vol. 3, pp. 1-17.

[29] H. A. Frank and R. J. Cogdell, "Carotenoids in Photosynthesis," Photochem. Photobiol., vol. 63, no. 3, pp. 257-264, 1996.

[30] F. Fungo, L. Otero, E. Durantini, W. J. Thompson, J. J. Silber, T. A. Moore, A. L. Moore, D. Gust, and L. Sereno, "Correlation of fluorescence quenching in carotenoporphyrin dyads with the energy of intramolecular charge transfer states. Effect of the number of conjugated double bonds of the carotenoid moiety," Phys. Chem. Chem. Phys., vol. 5, no. 3, pp. 469-475, 2003.

[31] P. S. Rukin, P. A. Kashchenko, A. Y. Malyavskaya, A. A. Bagaturyants, and M. V Alfimov, "Experimental and Theoretical Study of the Interaction of Volatile Amines with Zinc Porphyrins," vol. 9 , pp. 136-144, 2014.

[32] A. N. Macpherson, P. A. Liddell, S. Lin, L. Noss, G. R. Seely, J. M. Degraziano, A. L. Moore, T. A. Moore, and D. Gust, "Ultrafast Photoinduced Electron Transfer in Rigid Porphyrin - Quinone Dyads," no. 11, pp. 7202-7212, 1995.

[33] Y. Terazono, G. Kodis, P. A. Liddell, V. Garg, M. Gervaldo, T. A. Moore, A. L. Moore, and D. Gust, "Rapid Communication Photoinduced Electron Transfer in a Hexaphenylbenzene-based Selfassembled Porphyrin-fullerene Triad," no. li, pp. 464-469, 2007.

[34] J. Ravensbergen, A. Antoniuk-Pablant, B. D. Sherman, G. Kodis, J. D. Megiatto, D. D. MéndezHernández, R. N. Frese, R. Van Grondelle, T. A. Moore, A. L. Moore, D. Gust, and J. T. M. Kennis, "Spectroscopic Analysis of a Biomimetic Model of Tyr<inf $>Z</$ inf $>$ Function in PSII," J. Phys. Chem. B, vol. 119, no. 37, pp. 12156-12163, 2015.

[35] A. Antoniuk-Pablant, G. Kodis, A. L. Moore, T. A. Moore, and D. Gust, "Photoinduced electron and energy transfer in a molecular triad featuring a fullerene redox mediator," In Print, 2015.

[36] R. E. Palacios, G. Kodis, C. Herrero, E. Marin, M. Gervaldo, S. L. Gould, J. T. M. Kennis, D. Gust, T. A. Moore, and A. L. Moore, "Tetrapyrrole Singlet Excited State Quenching by Carotenoids in an Artificial Photosynthetic," pp. 25411-25420, 2006.

[37] G. Kodis, C. Herrero, R. Palacios, E. Marin, S. Gould, L. De Garza, R. Van Grondelle, D. Gust, T. A. Moore, A. L. Moore, and J. T. M. Kennis, "Light Harvesting and Photoprotective Functions of Carotenoids in Compact Artificial Photosynthetic Antenna Designs," pp. 414-425, 2004.

[38] Y. Terazono, G. Kodis, P. A. Liddell, V. Garg, T. A. Moore, A. L. Moore, and D. Gust, "Multiantenna artificial photosynthetic reaction center complex," J. Phys. Chem. B, vol. 113, no. 20, pp. 7147-7155, 2009 .

[39] A. L. Moore, A. Joy, R. Tom, D. Gust, T. A. Moore, V. Rene, and E. J. Land, "Energy Transfer," vol. 216, no. 4549, pp. 982-984, 2016.

[40] R. L. Milot, G. F. Moore, R. H. Crabtree, G. W. Brudvig, and C. A. Schmuttenmaer, "Electron injection dynamics from photoexcited porphyrin dyes into $\mathrm{SnO} 2$ and TiO2 nanoparticles," J. Phys. Chem. C, vol. 117, no. 42, pp. 21662-21670, 2013.

[41] K. Ohkubo, H. Imahori, J. Shao, Z. Ou, K. M. Kadish, Y. Chen, G. Zheng, R. K. Pandey, M. Fujitsuka, and O. Ito, "Small Reorganization Energy of Intramolecular Electron Transfer in Fullerene-Based Dyads with Short Linkage," pp. 10991-10998, 2002.

[42] I. R. Could, J. E. Maser, and S. Farid, "Efficiencies of Photoinduced Electron-Transfer Reactions: Role 
of the Marcus Inverted Region in Return Electron Transfer within Geminate Radical-Ion Pairs," no. d, pp. 4290-4301, 1990.

[43] G. S. H. P. Pan Xia, Zhiyuan Huang, Xin Li, Juan J. Romero, Valentine I. Vullev and M. L. Tang, "On the efficacy of anthracene isomers for triplet transmission from CdSe nanocrystals," 2016.

[44] V. Groenewegen, V. Kuntermann, D. Haarer, M. Kunz, and C. Kryschi, "Excited-state relaxation dynamics of 3-vinylthiophene-terminated silicon quantum dots," J. Phys. Chem. C, vol. 114, pp. 11693-11698, 2010.

[45] A. Sommer, C. Cimpean, M. Kunz, C. Oelsner, H. J. Kupka, and C. Kryschi, "Ultrafast excitation energy transfer in vinylpyridine terminated silicon quantum dots," J. Phys. Chem. C, vol. 115, pp. 22781-22788, 2011.

[46] R. Berera, R. van Grondelle, and J. T. M. Kennis, "Ultrafast transient absorption spectroscopy: Principles and application to photosynthetic systems," Photosynth. Res., vol. 101, no. 2-3, pp. 105118, 2009.

[47] S. and P. Y. Lee, I; Lee, "Excited-State Dynamics of Carotenoids Studied by Femtosecond Transient," vol. 35, no. 3, pp. 851-857, 2014.

[48] T. Gillbro and R. J. Cogdell, "Carotenoid fluorescence," Chem. Phys. Lett., vol. 158, no. 3-4, pp. 312316, 1989.

[49] K. Yasushi, "New trends in photobiology. Structures and functions of carotenoids in photosynthetic systems," J. Photochem. Photobiol. B Biol., vol. 9, no. 3-4, pp. 265-280, 1991.

[50] C. C. Gradinaru, J. T. M. Kennis, E. Papagiannakis, I. H. M. Van Stokkum, R. J. Cogdell, G. R. Fleming, R. A. Niederman, and R. Van Grondelle, "An unusual pathway of excitation energy deactivation in carotenoids : Singlet-to-triplet conversion on an ultrafast timescale in a photosynthetic antenna," 2000.

[51] D. Zigmantas, R. G. Hiller, F. P. Sharples, and H. A. Frank, "Effect of a conjugated carbonyl group on the photophysical properties of carotenoids," pp. 3009-3016, 2004.

[52] N. E. Holt, D. Zigmantas, and L. Valkunas, "Carotenoid Cation Formation and the Regulation of Photosynthetic Light Harvesting," vol. 307, no. January, 2005.

[53] R. E. Palacios, G. Kodis, C. Herrero, E. M. Ochoa, S. L. Gould, J. T. M. Kennis, D. Gust, T. A. Moore, and A. L. Moore, "Tetrapyrrole Singlet Excited State Quenching by Carotenoids in an Artificial Photosynthetic Antenna," pp. 1-6.

[54] J. D. Megiatto, A. Antoniuk-Pablant, B. D. Sherman, G. Kodis, M. Gervaldo, T. A. Moore, A. L. Moore, and D. Gust, "Mimicking the electron transfer chain in photosystem II with a molecular triad thermodynamically capable of water oxidation," Proc. Natl. Acad. Sci., vol. 109, no. 39, pp. 1557815583, 2012.

[55] P. Persson and R. Bergstro, "Quantum Chemical Study of Photoinjection Processes in Dye-Sensitized TiO 2 Nanoparticles," pp. 10348-10351, 2000.

[56] K. Tvrdy, P. A. Frantsuzov, and P. V Kamat, "Photoinduced electron transfer from semiconductor quantum dots to metal oxide nanoparticles," vol. 108, no. 1, 2011.

[57] M. Kuno and P. V Kamat, "Size-Dependent Electron Injection from Excited CdSe Quantum Dots into 
TiO 2 Nanoparticles," pp. 4136-4137, 2007. 
- 145 - 


\section{Capítulo 7: Regulación de propiedades ópticas.}

\section{Ensamblados coloidales con Au nps}

En este capítulo se reporta la síntesis, caracterización y propiedades fotofísicas de ensamblados coloidales que involucran Au nps y Si nps. Se emplearon Au nps de diversos tamaños, a fin de explorar distintas condiciones en las cuales se manifiesten propiedades mejoradas por metales. Se encontró una mejora interesante en los QY de PL y en la producción de oxígeno singlete, lo cual hace que estos sistemas sean buenos candidatos para continuar con su desarrollo como posibles fotosensibilizadores.

\subsection{Introducción}

Las reacciones fotoquímicas juegan un rol importante tanto en la naturaleza como a nivel tecnológico, formando parte desde el proceso de fotosíntesis hasta de técnicas de depuración de aguas y transducción de energía solar [1]. Estas reacciones involucran estados excitados que ofrecen vías de reacción alternativas, las cuales conducen a productos diferentes o incluso inaccesibles comparadas a sus contrapartes térmicas. Sin embargo, el control preciso de los estados involucrados no es una tarea fácil. En este aspecto, la excitación de nanoestructuras metálicas en el entorno del cromóforo de interés pueden generar campos eléctricos intensos y altamente localizados [2], logrando poblar en mayor medida estados excitados determinados que conduzcan a procesos fotoquímicos controlados [3]-[6]. Esta estrategia ha sido utilizada exitosamente tanto en el aumento de señales Raman como en señales fluorescentes [7]. En este capítulo, se presentan resultados sobre la interacción de coloides de oro (Au nps), y nanopartículas de Silicio (Si nps).

Preparamos los coloides de Oro por crecimiento de semillas usando hidroxilamina [8]. Por su parte, las Si nps se obtuvieron por oxidación de siliciuros metálicos [9] y se funcionalizaron con grupos específicos usando reacciones de hidrosililación. Los ensamblados fueron preparados por mezcla directa de sus bloques de construcción. Se caracterizaron empleando TEM, FTIR y XPS, y se evaluaron sus propiedades fotoquímicas usando espectroscopías de absorción UV-Visible y emisión, tanto estacionarias como resueltas en el tiempo.

En presencia de nanopartículas metálicas, se observan cambios importantes en el comportamiento de los cromóforos empleados, llevando al aumento del rendimiento cuántico de emisión y de generación de oxígeno singlete en el caso de Au@Si nps. A través de experimentos ópticos resueltos en el tiempo se obtuvieron evidencias para identificar los mecanismos que explican el comportamiento de cada sistema estudiado. 

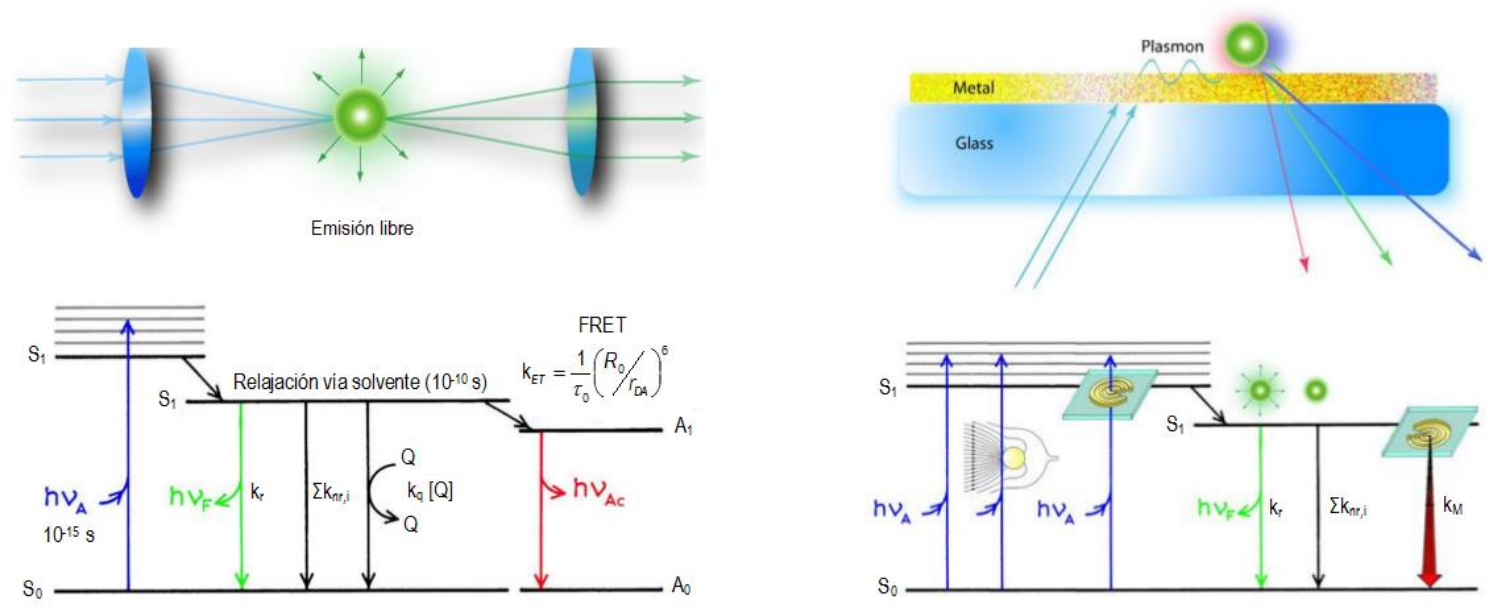

Figura 7.1. Diagramas de Jablonski para emisión en condiciones normales y en presencia de metales. Adaptado de [7]

\subsection{Aspectos sintéticos y metodológicos}

Los detalles referidos a síntesis y métodos experimentales han sido desarrollados en mayor extensión en los capítulos 2 y 3 . A continuación, se comentarán de forma breve los procedimientos relacionados al presente capítulo.

Las Si nps estudiadas en este capítulo fueron sintetizadas usando una adaptación de las técnicas publicadas por Kauzlarich y colaboradores [9][10][11]. Los detalles correspondientes a esta síntesis se encuentran en las secciones 3.2.4 a 3.2.7. del capítulo 3. A su vez, la obtención, caracterización y propiedades ópticas de PA-BU' Si nps de distintos tamaños se detalla en el capítulo 5. Brevemente, en un balón de 3 bocas con un refrigerante adosado, se colocaron $120.00 \mathrm{ml}$ de DMF anhidro, que fueron calentados hasta $100^{\circ} \mathrm{C}$ y purgados con $\mathrm{Ar}$ durante $30 \mathrm{~min}$. Luego, se agregaron $100.0 \mathrm{mg}$ de $\mathrm{Mg}_{2} \mathrm{Si}$ finamente molido y el sistema se mantuvo en calentamiento y purga hasta que alcance el reflujo. En el paso siguiente, se adicionaron al sistema $200.0 \mathrm{mg}$ de $\mathrm{NH} 4 \mathrm{Cl}$ disueltos en $10.00 \mathrm{ml}$ de DMF y se continuó el calentamiento hasta la condición de reflujo, que se mantuvo por 20 hs. Finalmente, el sistema se dejó enfriar a temperatura ambiente. En estas condiciones se obtuvieron BU' Si nps de $(3.4 \pm 0.6)$, las cuales fueron purificadas usando diálisis. La suspensión obtenida se separó en 2 alícuotas. En el primer caso, se evaporó el solvente y se agregaron $25.00 \mathrm{ml}$ de agua ultrapura, obteniendosé una suspensión acuosa de BU' Si nps. En el segundo caso, se evaporó el solvente usando un evaporador rotatorio y la muestra fue disuelta en $25.00 \mathrm{ml}$ de tolueno, y purgada durante 30 min usando Ar. Posteriormente se agregaron $250 \mu \mathrm{l}$ de AA y la muestra fue irradiada usando 8 lámparas de $254 \mathrm{~nm}$ durante 5 hs. Nuevamente, las PA-BU' Si nps obtenidas se purificaron usando diálisis. Finalmente, el solvente se removió evaporando a presión reducida y la muestra se redisolvió en $25.00 \mathrm{ml}$ de agua ultrapura.

Las Au nps empleadas en este capítulo se sintetizaron por el método de crecimiento de semillas usando hidroxilamina. El protocolo sintético se detalló en la sección 3.4. del capítulo 3. Las semillas se sintetizaron usando el método de Turkevich - Frens [12], [13]. En un balón de reacción se colocaron $65.00 \mathrm{ml}$ de una solución acuosa fresca de $\mathrm{HAuCl}_{4} 254 \mu \mathrm{M}$ y se calentó a ebullición con agitación vigorosa. Se agregaron 6.50 $\mathrm{ml}$ de citrato de sodio $40 \mathrm{mM}$ y la solución paso de ser amarilla a roja, color propio de las Au nps. La mezcla se mantuvo en ebullición por 10 minutos y luego se dejó enfriar a temperatura ambiente. Estas Au nps de (32 \pm 10) $\mathrm{nm}$ se purificaron por diálisis contra agua ultrapura, y almacenadas a $4 \mathrm{C}$. Posteriormente estas muestras se calentaron hasta temperatura ambiente, y en agitación vigorosa se agregaron distintos volúmenes de soluiciones de $\mathrm{NH} 2 \mathrm{OH}$ y HAuCl4, para hacer crecer las semillas [13], [14]. Las cantidades empleadas de semillas, $\mathrm{HAuCl} 4$ y NH2OH definen el tamaño final de las Au nps. Para obtener Au nps de $(56 \pm 10) \mathrm{nm}$ se colocaron $7.50 \mathrm{ml}$ de semillas Au nps de $(32 \pm 10) \mathrm{nm}$ de concentración $3 \mathrm{nM}$ junto a $4.00 \mathrm{ml}$ de $\mathrm{NH}_{2} \mathrm{OH} 40 \mathrm{mM}$ 
y se completó con agua ultrapura hasta $75.00 \mathrm{ml}$. Esta mezcla se agitó a temperatura ambiente, y luego se agregaron $100 \mu \mathrm{l}$ de $\mathrm{HAuCl}_{4} 254 \mu \mathrm{M}$. Se observó un cambio inmediato en la tonalidad de la dispersión de Au nps. Posteriormente, se dializó contra agua ultrapura. Para obtener Au nps de $(87 \pm 12) \mathrm{nm}$ se emplearon las mismas cantidades de semillas y reductor $300 \mu \mathrm{l}$ de $\mathrm{HAuCl}_{4} 254 \mu \mathrm{M}$.

Usando las dispersiones coloidales de Si nps y Au nps, se prepararon 2 tipos de ensamblados: Blanco, empleando BU' Si nps con Au nps de distintos diámetros d, los cuales serán denominados Au(d)@BU' Si nps; y sistema, empleando PA-BU'Si nps nuevamente con Au nps de distintos diámetros d, estos serán denominados Au(d)@PA-BU' Si nps.

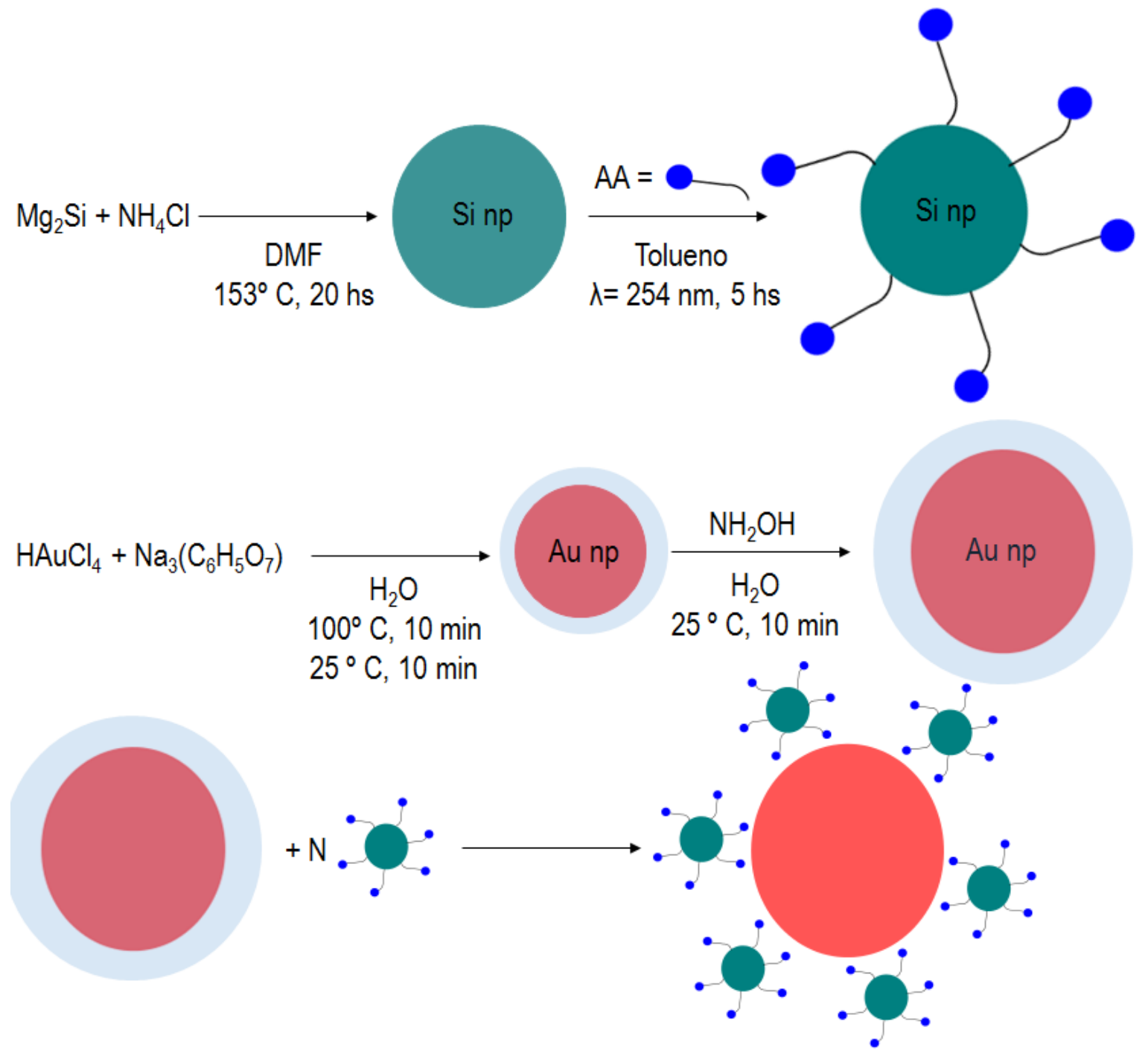

Figura 7.2. Esquemas de síntesis empleados. Los gráficos son solo a fines ilustrativos.

Los ensamblados se prepararon agregando sobre un volumen conocido de una de dispersión madre de Au nps de diámetro d, cuya concentración se estimó usando el espectro de atenuación UV-vis del coloide, como se explicó en la sección 3.4.4 [8], volúmenes crecientes de dispersiones de BU' Si nps o PA-BU' Si nps, cuya concentración analítica se determinó usando espectrometría de emisión atómica (ICP - AES) y datos obtenidos por TEM. Este método se detalla en la sección 3. La nomenclatura utilizada a lo largo de este capítulo para las distintas nps y ensamblados estudiados se detalla en la siguiente tabla. 


\begin{tabular}{ccc}
\hline Si nps & Au nps & Nombre \\
\hline BU' Si nps & Au 32 & Au(32)@Si \\
& Au 56 & Au(56)@Si \\
& Au 87 & Au(87)@Si \\
PA-BU' Si nps & Au 32 & Au(32)@paSi \\
& Au 56 & Au(56)@paSi \\
& Au 87 & Au(87)@paSi
\end{tabular}

Tabla 7.1. Nomenclatura utilizada para los ensamblados estudiados en este capítulo.

\subsection{Resultados y discusión}

\subsubsection{Síntesis y caracterización}

Se sintetizaron exitosamente Si nps de $(3.6 \pm 0.6) \mathrm{nm}$ por oxidación de $\mathrm{Mg}_{2} \mathrm{Si}$ y Au nps de distintos tamaños usando el método de Turkevich - Frens para las semillas, y crecimiento por reducción de $\mathrm{HAuCl}_{4}$ con hidroxilamina. La caracterización detallada de las distintas Si nps usadas en este capítulo se detalla en el capítulo 5. Las Au nps sintetizadas fueron caracterizadas usando TEM, XPS y espectroscopía UV-vis., al igual que los ensamblados coloidales Au@Si. Los resultados obtenidos se comentan a continuación.

Como primera aproximación, se midieron y analizaron los espectros de atenuación UV-vis de los coloides de $\mathrm{Au}$, a fin de obtener información cualitativa y cuantitativa. Todas las medidas fueron realizadas en suspensiones $1 \mu \mathrm{M}$ de citrato de sodio (que actúa como ligando), a fin de mantener las Au nps suspendidas en el medio. El comportamiento observado en los espectros UV-vis se corresponde con el esperado a partir de las soluciones de Mie de las ecuaciones de Maxwell, observándose un corrimiento al rojo de la banda de plasmones superficiales (SPB, del inglés Surface Plasmon Band) a mayor tamaño de las nanopartículas. La aplicación de las soluciones de Mie en cálculos para predecir propiedades ópticas es un campo activo de la investigación en nanociencia [2], y que puede aplicarse para obtención de datos analíticos. Basados en el método desarrollado por Haiss y colaboradores [8], se estimó el tamaño de las Au nps y su concentración. Los resultados de la misma se presentan en la siguiente tabla, comparados con medidas estándar para la deteminación de estas magnitudes.

\begin{tabular}{cccccccc}
\hline Muestra & $\lambda_{\max }(\mathrm{nm})$ & $\mathrm{d}_{\mathrm{uv}-\mathrm{vis}}(\mathrm{nm})$ & $\langle\mathrm{d}\rangle_{\text {TEM }}(\mathrm{nm})$ & $\mathrm{e}_{\mathrm{r}, \mathrm{d}}(\%)$ & $\mathrm{C}_{\mathrm{uv}-\mathrm{vis}}(\mathrm{M})$ & $\mathrm{C}_{\text {ICP }}(\mathrm{M})$ & $\mathrm{e}_{\mathrm{r}, \mathrm{C}}(\%)$ \\
\hline $\mathrm{Au}(32)$ & 524.5 & 30.12 & 32 & 5.88 & $1.62 \mathrm{E}-13$ & $2.10 \mathrm{E}-13$ & 22.9 \\
$\mathrm{Au}(56)$ & 560.0 & 46.99 & 56 & 16.11 & $3.00 \mathrm{E}-14$ & $3.50 \mathrm{E}-14$ & 14.4 \\
$\mathrm{Au}(87)$ & 571.5 & 99.25 & 87 & 14.08 & $6.74 \mathrm{E}-15$ & $7.36 \mathrm{E}-15$ & 8.49
\end{tabular}

Tabla 7.2. Estimación de tamaños y concentración de coloides para Au, a partir de medidas de atenuación UV-vis. Para la estimación de los errores relativos, se consideraron medidas de referencia TEM para determinación de $<\mathrm{d}>$ e ICP para $\mathrm{C}$. 


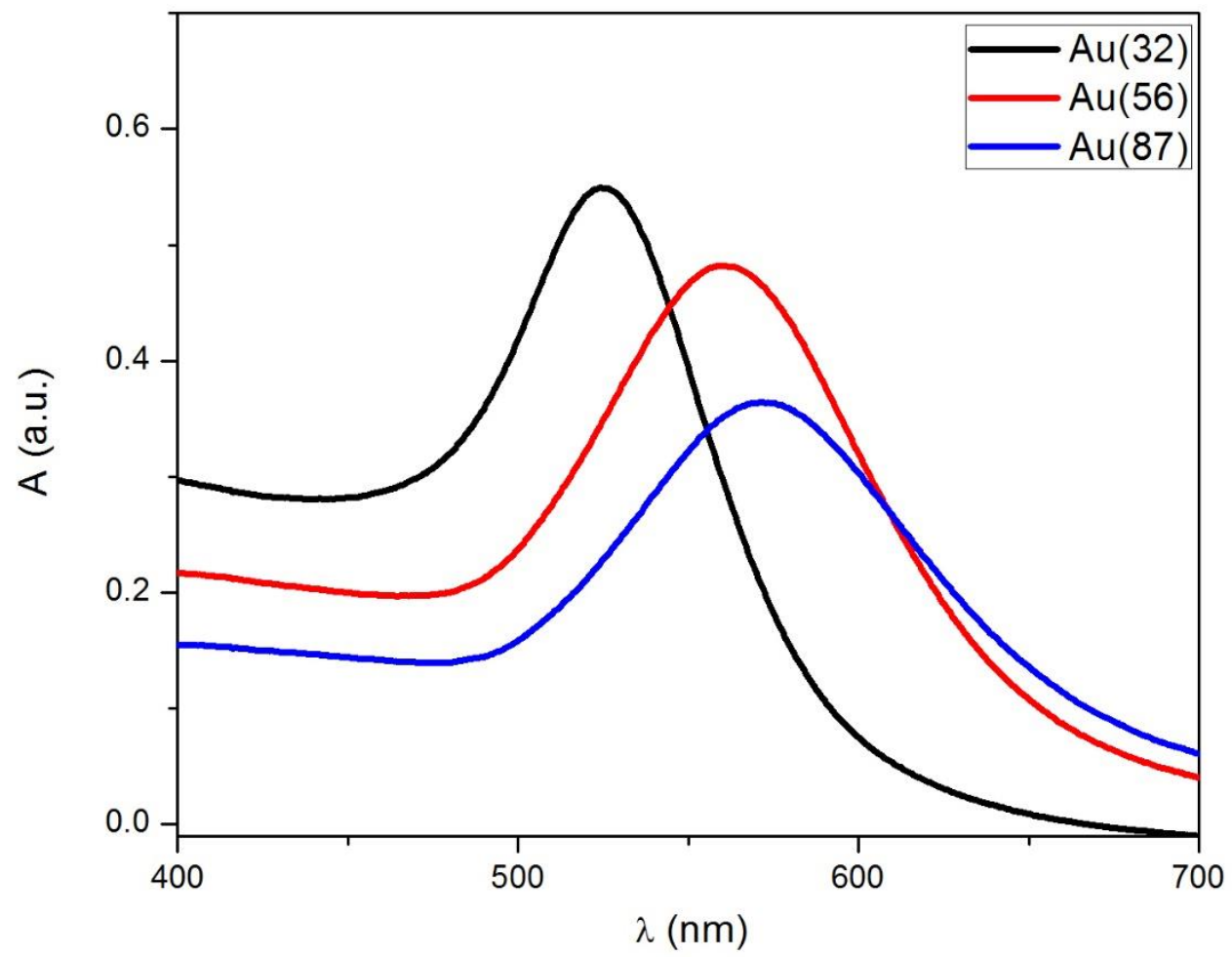

Figura 7.3. Espectros de atenuación UV-vis de los coloides $\mathrm{Au}(32), \mathrm{Au}(56)$ y $\mathrm{Au}(87)$.

Utilizando medidas de TEM se obtuvieron las distribuciones de tamaños de las muestras de Au nps preparadas, obteniéndose resultados aceptables. Para las Au(32) sintetizadas por el método de Turkevich - Frens se obtuvo una distribución de $(32 \pm 10) \mathrm{nm}$. En las imágenes obtenidas con alta resolución (HR-TEM) puede observarse la cristalinidad del producto, pudiendo diferenciarse nps monocristalinas y policristalinas. Las transformadas de Fourier de las imágenes obtenidas para nps cristalinas coinciden con lo obtenido para cristales de Au FCC, y en el caso de partículas policristalinas se observa el mismo arreglo de puntos pero de forma difusa, lo cual refuerza lo observado de forma directa en las imágenes. También se realizaron experimentos de difracción de electrones para confirmar lo estimado usando transformadas de Fourier, obteniéndose los resultados esperados.

Las $\mathrm{Au}(32)$ se utilizaron como semillas para el crecimiento de nps de mayor tamaño, por reducción de $\mathrm{HAuCl}_{4}$ con hidroxilmina, usando agregados controlados de estos dos reactivos. Las Au nps obtenidas por crecimiento fueron $\mathrm{Au}(56)$ con tamaños de $(56 \pm 10) \mathrm{nm}$ y $\mathrm{Au}(87)$ de $(87 \pm 12) \mathrm{nm}$. En estos casos, las nps son el producto de la aglomeración y crecimiento de capas cristalinas sobre un conjunto de nanopartículas Au(32), como puede verse claramente en la figura 7.2. En consecuencia, las muestras son policristalinas y a su vez la distribución de tamaños conserva su varianza muestral. Para poder mejorar este aspecto y lograr nps con mayor grado de cristalinidad y distribuciones más estrechas, puede optar por aumentarse la temperatura de síntesis y y trabajar en condiciones donde la digestión de Ostwald sea el proceso favorecido. 

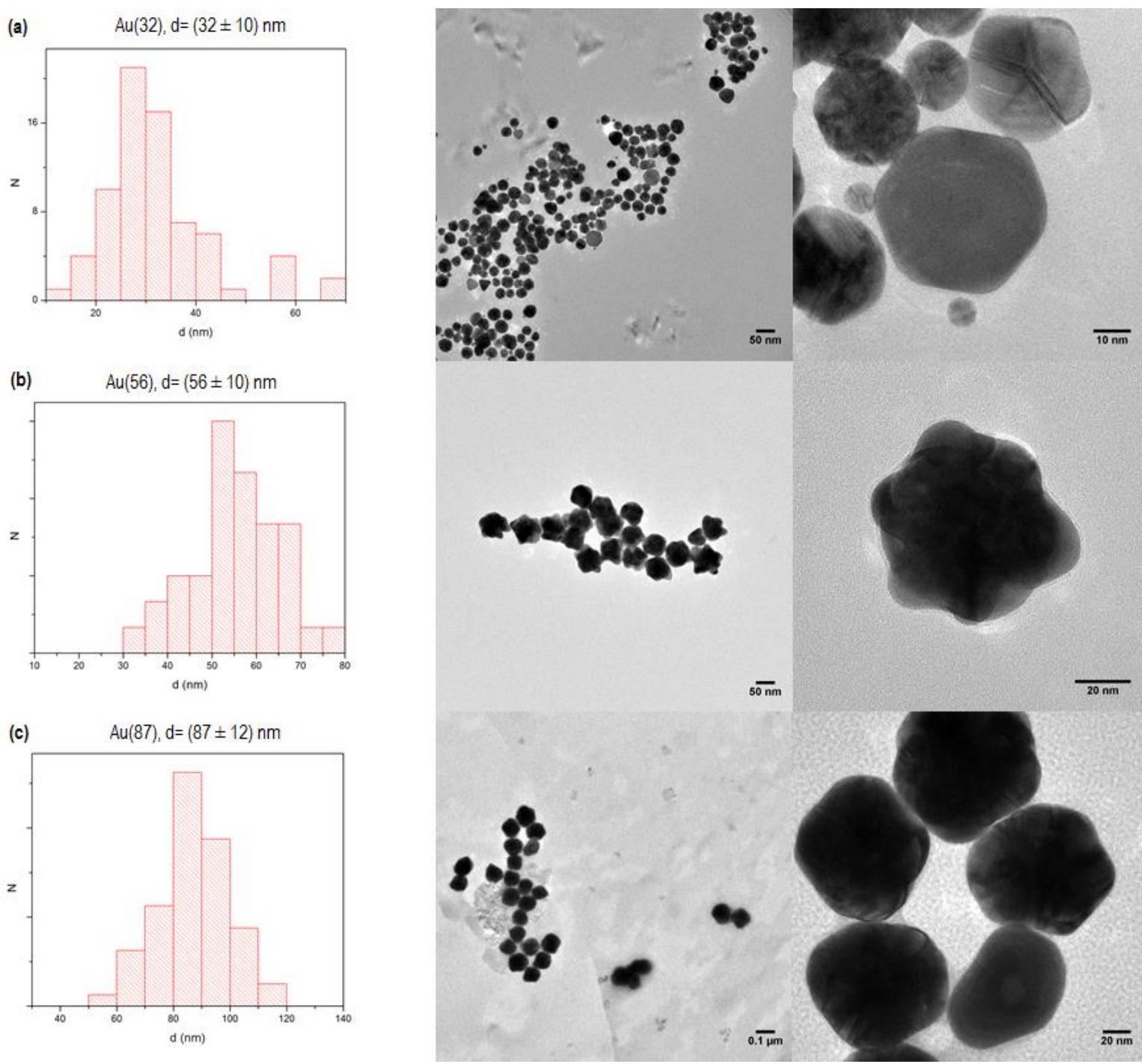

Figura 7.4. Distribuciones de tamaños, micrografías TEM y HR-TEM de (a) $A u(32)$, (b) $A u(56)$ y (c) $A u(87)$.

Para estudiar la formación de ensamblados coloidales entre Au nps y Si nps, sobre muestras de los distintos coloides de Au se agregaron volúmenes crecientes de Si nps, tanto cubiertas con propil amina (Estos ensamblados se denominaron "sistema") como oxidadas (Ensamblados denominados "blanco"). Sobre un volumen conocido, constante y de concentración conocida de las muestras de Au nps agregamos volúmenes sucesivos de una dispersión madre de Si nps. La evolución de los espectros de atenuación UV-vis se muestra en la figura 7.5, y su composición en la tabla 7.3.

\begin{tabular}{cccc}
\hline $\mathrm{C}_{\text {Si nps }}(\mathrm{uM})$ & nSi:nAu (Au32) & nSi:nAu (Au56) & nSi:nAu (Au87) \\
\hline 0 & 0 & 0 & 0 \\
1.311 & 69536.0221 & 570102.667 & $5.49 \mathrm{E}+06$ \\
3.786 & 208608.066 & $1.71 \mathrm{E}+06$ & $1.65 \mathrm{E}+07$ \\
6.080 & 347680.111 & $2.85 \mathrm{E}+06$ & $2.74 \mathrm{E}+07$
\end{tabular}




$\begin{array}{llll}8.213 & 486752.155 & 3.99 \mathrm{E}+06 & 3.84 \mathrm{E}+07 \\ 11.14 & 695360.221 & 5.70 \mathrm{E}+06 & 5.49 \mathrm{E}+07 \\ 19.11 & 1.39 \mathrm{E}+06 & 1.14 \mathrm{E}+07 & 1.10 \mathrm{E}+08 \\ 29.72 & 2.78 \mathrm{E}+06 & 2.28 \mathrm{E}+07 & 2.20 \mathrm{E}+08 \\ 33.43 & 3.48 \mathrm{E}+06 & 2.85 \mathrm{E}+07 & 2.74 \mathrm{E}+08\end{array}$

Tabla 7.3. Concentraciones y relaciones Si:Au empleadas en los experimentos informados en este capítulo. Las concentraciones de Au nps se informaron en la tabla 7.2.

Para cada caso (ensamblados blanco y sistema), los espectros obtenidos son distintos a los espectros suma de los espectros individuales de los bloques de construcción. Esto indica que existe una interacción entre ellos que altera la superficie de energía potencial del sistema, y provoca cambios en el espectro de absorción.

En el caso de ensamblados blanco, solo se observó un corrimiento al rojo de la SPB, mientras que los cambios en los ensamblados sistema fue más complejo. Se observó, en primer término, un corrimiento al rojo de la SPB, junto con la aparición de una banda en la región entre 600 y $800 \mathrm{~nm}$. El corrimiento de la SPB es consistente con un cambio en el entorno dieléctrico de las Au nps. Esto puede ocurrir por cambios en la composición de la doble capa eléctrica, ocasionado por el desplazamiento de iones que se adsorban de forma inespecífica o por el agregado de ligandos multidentados que favorezcan la interacción de múltiples Au nps [15]. Este último caso soportado por la aparición de una banda en la región de 600 a $800 \mathrm{~nm}$ del espectro UV-vis de los ensamblados al aumentar el agregado de PA-BU' Si nps, y se observa de forma extendida en bibliografía para interacción de coloides de Au con ligandos del tipo diamina [16][17]. A fin de corroborar la formación de estos complejos de agregación y estudiar su composición, se realizaron medidas de TEM y XPS.
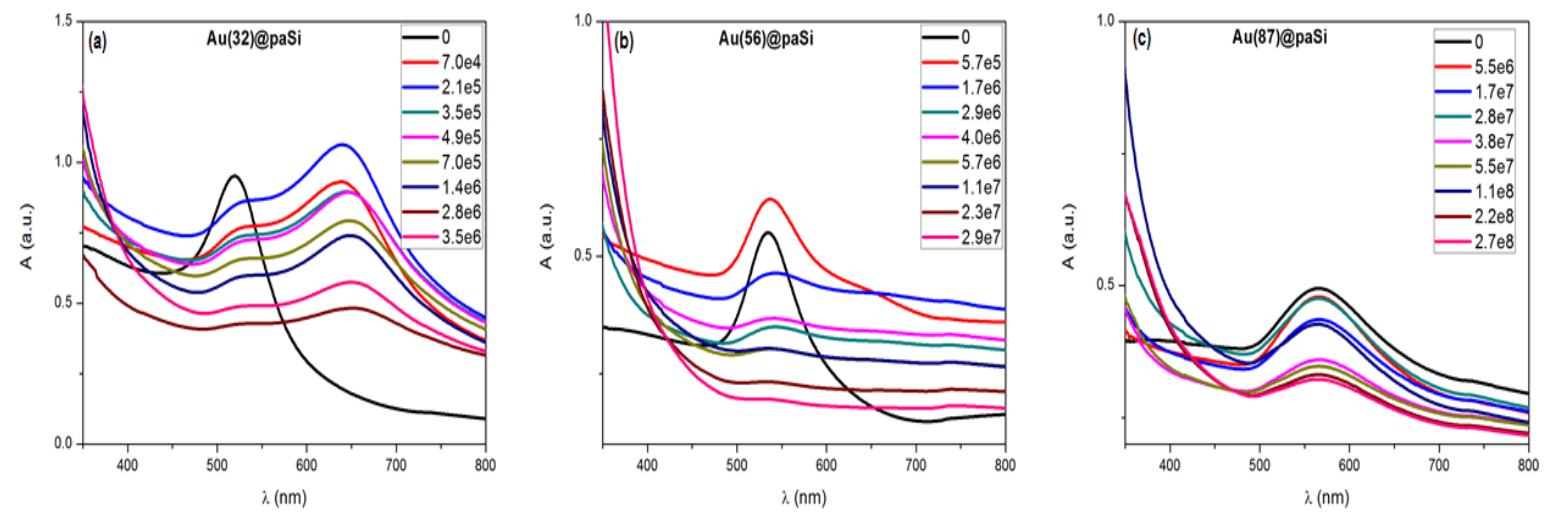

Figura 7.5. Evolución de los espectros UV-vis para los ensamblados (a) Au(32)@paSi, (b) Au(56)@paSi y (c) Au(87)@paSi. Los distintos colores de línea muestran el aumento en la relación nsi/nAu.

Al estudiar las micrografías de campo claro obtenidas por TEM se corroboró la formación de agregados, tanto en ensamblados blanco como sistema, comparados a las Au nps en ausencia de Si nps. En el caso de Au nps solas, se observaron claramente dispersas a lo largo de la grilla (figura 7.6.a). Sin embargo, al recorrer las grillas para ensamblados se observó claramente la formación de agregados, los cuales son distintos según sean ensamblados blanco o sistema. En ensamblados blanco (figura7.6.b) se encontraron aglomerados de entre 30 y $50 \mathrm{nps}$ los cuales no mostraron una clara organización, a diferencia de lo observado para ensamblados sistema, que presentan mayor extensión y estructura tridimensional clara (figura7.6.c). Estos detalles soportan la interpretación de las bandas observadas en los espectros UV-vis al agregar Si nps sobre los coloides de Au. Para obtener mayor información sobre el rol de las Si nps en la formación de aglomerados, se obtuvieron imágenes de HR-TEM complementadas con experimentos de EDS, para corroborar la identidad de las distintas nps. 


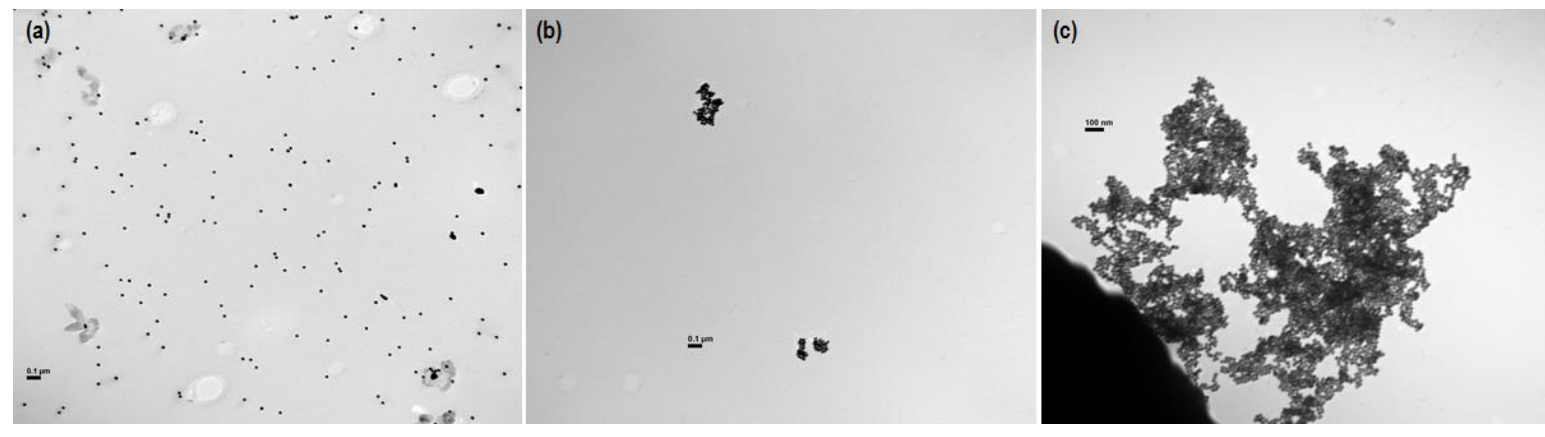

Figura 7.6. Imágenes TEM de campo claro para (a) Au(32) (b) Au(32)@Si y (c) Au(32)@paSi.

En estos experimentos se prestó mayor atención en el contacto entre Au nps y la presencia de nps más pequeñas de Si en su entorno. En principio, esta tarea fue un tanto desafiante, debido a la diferencia de contrastes para TEM entre Si y Au. Si bien se encontraron Au nps rodeadas de nps más pequeñas, no pudo asignarse identidad de forma unívoca. Usando experimentos de EDS se corroboró la presencia de ambos materiales en las zonas estudiadas. Sin embargo los resultados de la distribución de elementos no resultó concluyente. Del mismo modo, al observar la presencia de nps pequeñas en el entorno de Au nps, las transformadas de Fourier de estas no coindice con los patrones observados para $\mathrm{Au}, \mathrm{Si} 0 \mathrm{SiO}_{2}$ con lo cual el resultado no puede considerarse como concluyente. 

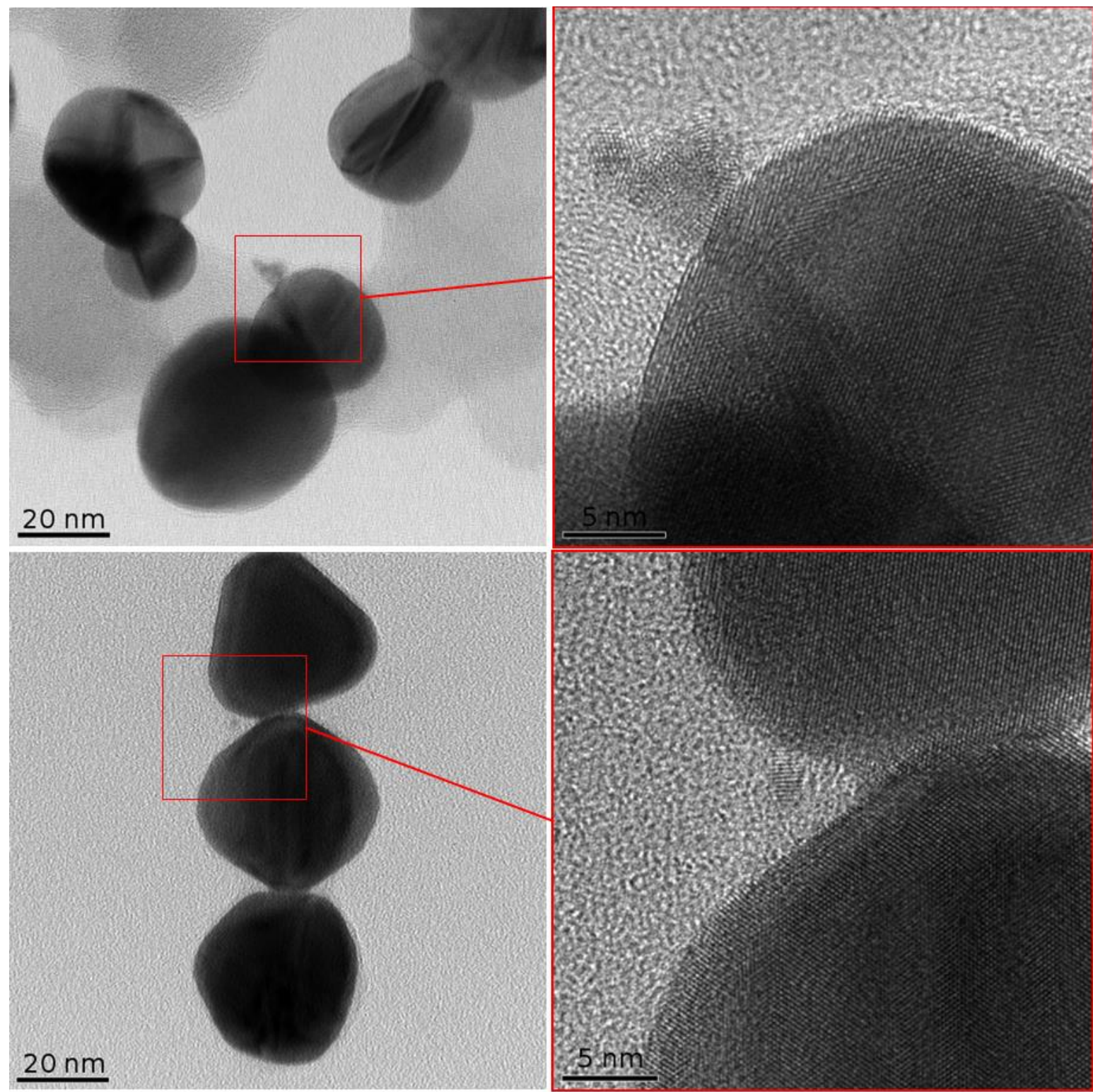

Figura 7.7. Imágenes HR-TEM de campo claro para ensamblados Au(32)@paSi, en los cuales se destaca la presencia de nps más pequeñas. Sin embargo, la identidad de estas nps más pequeñas no pudo asignarse unívocamente.

La composición de los ensamblados se estudió usando XPS y de forma complementaria FTIR. Para estudiar estas muestras, los experimentos de XPS se realizaron depositando unas gotas de las dispersiones correspondientes sobre un soporte de C, mientras que para estudios de FTIR se usaron como soportes cristales de $\mathrm{Si}$, a fin de obtener información sobre los ligandos carbonados que cubren las Au nps. Con esto en mente, se prestó atención principalmente al ajuste de la señal de Au en el caso de XPS, para luego observar su comportamiento en los ensamblados Au@Si, tomando los ajustes para Au nps como referencia. 

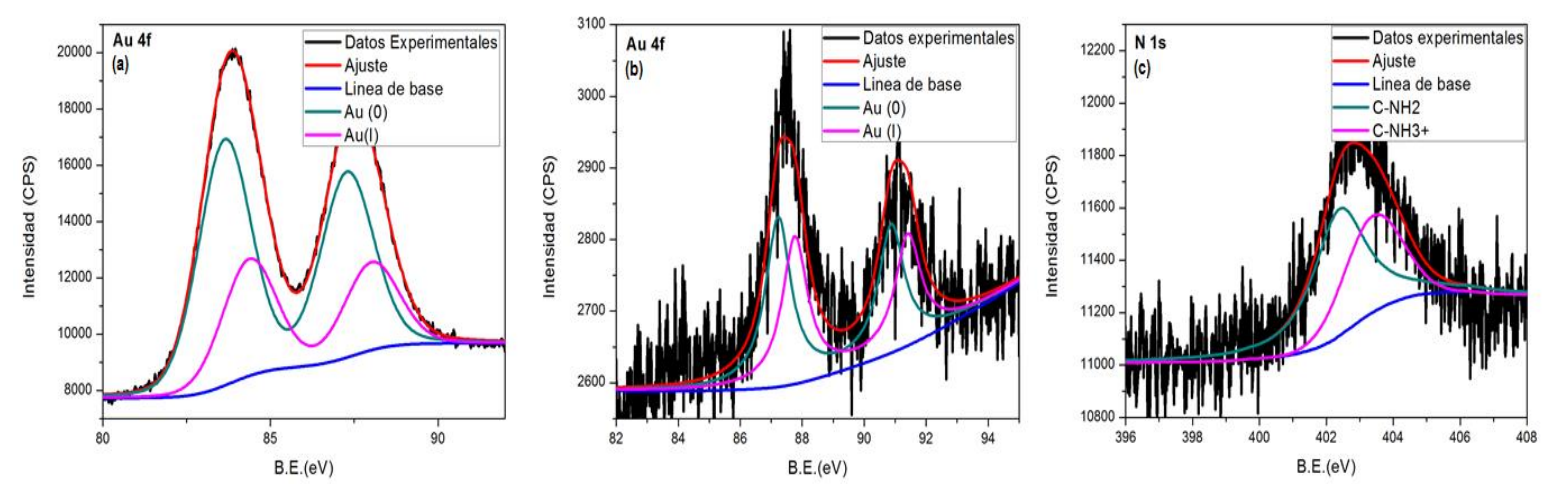

Figura 7.8. Espectros XPS de alta resolución de los coloides de Au, en las regiones Au 4f y N 1s. Los espectros corresponden a (a) $\mathrm{Au}(87)$, (b) y (c) ensamblado Au(87)@paSi.

Las medidas de FTIR se realizaron a fin de comprender el rol del citrato en los coloides de Au. Luego de distintos ensayos de diálisis contra solvente puro, se observó que los iones citrato actúan de ligandos estabilizantes de las Au nps, con lo cual los pasos de purificación final por diálisis solo se realizaron por intercambios con solvente puro por una hora. La interacción de iones citrato con la superficie de las Au nps puede estar mediada por iones $\mathrm{Au}(\mathrm{I})$ presentes, los cuales han sido propuestos a lo largo de la literatura [18], [19].

Al estudiar los espectros XPS en la región de Au 4f, la señal detectada puede asignarse como una suma de contribuciones de $\mathrm{Au}(0)$ y $\mathrm{Au}(\mathrm{I})$, las cuales se manifiestan en el entorno de $84 \mathrm{eV}$ y $84.6 \mathrm{eV}$, respectivamente. Para las Au nps cubiertas por citratos, las posiciones de los picos se mantuvieron en este entorno, presentando corrimientos mínimos atribuibles al error experimental. Sin embargo, en el caso de ensamblados se registraron casos diferentes. Para los ensamblados blanco no se detectaron cambios significativos, mientras que en los ensamblados sistema no solo se obseró un corrimiento en las señales sino que una disminución en la relación de áreas $\mathrm{Au}(0) / \mathrm{Au}(\mathrm{I})$. Considerando que los factores de sensibilidad de detección no cambian de experimento en experimento, el cambio en la relación de áreas puede explicarse con la generación de defectos de $\mathrm{Au}(\mathrm{I})$ inducidos por la presencia de PA-BU' Si nps, los cuales están vinculados al anclaje del grupo terminal amino con la superficie de las Au np. A su vez, al analizar la señal en la región de N 1s para los ensamblados sistema, estas pueden deconvolucionarse en 2 contribuciones en el entorno de 400 y $401 \mathrm{eV}$, asignadas a $\mathrm{N}$ en grupos $-\mathrm{NH}_{2}$ y $-\mathrm{NH}_{3}{ }^{+}$respectivamente. Con estos resultados en mente, se propone que la interacción entre PA-BU' Si nps y Au nps es por vía electrostática, mediada por complejos $\left[\mathrm{AuCl}_{2}\right]^{-}$presentes en la superficie. A su vez, no puede despreciarse la contribución de complejos de naturaleza covalente donde el grupo amino participe como ligando del complejo de $\mathrm{Au}(\mathrm{l})$.

$$
\mathrm{Au}_{n}\left[\mathrm{AuCl}_{2}\right]_{\mathrm{m}}{ }^{+}+\mathrm{m} \mathrm{NH}_{3}{ }^{+}-\mathrm{R}-\mathrm{Si} \mathrm{nps} \rightarrow \mathrm{Au}_{n}\left[\mathrm{AuCl}\left(\mathrm{NH}_{2}-\mathrm{R}-\mathrm{Si} \mathrm{nps}\right)\right]+\mathrm{HCl}
$$

Este mecanismo está muy vinculado al que ocurre por vía electrostática, y en principio para poder obtener información más precisa sobre la estructura de la interfase, es necesario realizar estudios en distintas condiciones de $\mathrm{pH}$, fuerza iónica y con distintos espaciadores $\mathrm{R}$, los cuales están más allá de los objetivos planteados en este capítulo.

Se ha reportado en bibliografía la síntesis de coloides de Au estabilizados con moléculas con grupos amino terminales, los cuales fueron caracterizados empleando diversas técnicas (incluida XPS) en los cuales se arribó a conclusiones similares [20], [21].

\subsubsection{Propiedades ópticas}

Teniendo en cuenta el proceso de aglomeración propuesto se estudiaron las propiedades ópticas relacionadas a la emisión de los ensamblados. Se prestó atención a las variaciones en el rendimiento cuántico de emisión $\Phi$, los tiempos de vida y la despolarización de la RE de la emisión a través de la anisotropía de emisión. Dada 
la proximidad entre el cromóforo y las Au nps, el estudio de las propiedades de emisión pueden aportar información sobre el efecto de la interacción entre estos sobre la estructura electrónica del cromóforo y la dinámica de relajación del estado excitado. El resultado de estas interacciones podría conducir a un efecto de emisión mejorada por metales, llamado en bibliografía MEF (del inglés Metal Enhanced Fluorescence)[22]. Este efecto es de gran importancia para fluoróforos de bajo $\Phi$, y constituye una de las estrategias actualmente empleadas para sintonizar propiedades ópticas y mejorar la sensibilidad de métodos analíticos.

En principio, los espectros de emisión estacionarios solo se vieron afectados en cuanto a intensidad y no a distribución espectral, lo cual indica que la presencia de Au nps no altera la densidad de probabilidad de decaimiento desde el estado excitado a los múltiples estados vibracionales aceptores en el estado fundamental. Por esta razón, se hizo foco en la variación de $\Phi$.

El efecto sobre $\Phi$ es distinto según sean las Au nps empleadas en la construcción del ensamblado. Al momento de considerar la posibilidad de MEF, $\Phi$ debería aumentar significativamente. Para estudiar esta posibilidad, se estudió la emisión de ensamblados con distintas relaciones $n_{\mathrm{Si}}: n_{\mathrm{Au}}$ para las distintas Au nps empleadas.
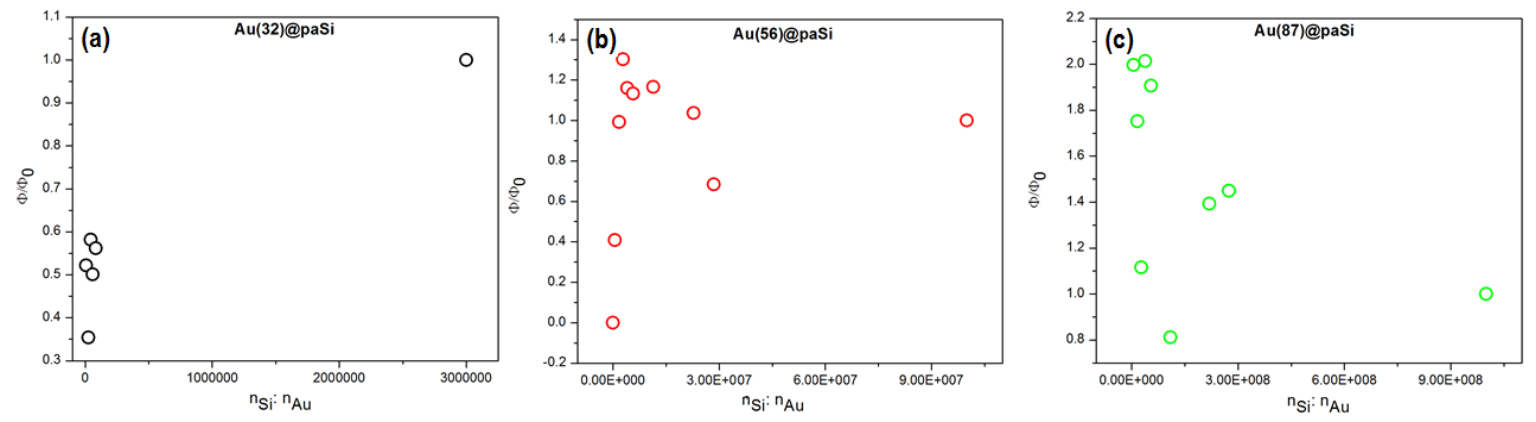

Figura 7.9. Variación de los rendimientos cuánticos de emisión (considerando como referencia PA-BU' Si nps) para (a) Au(32)@paSi, (b) Au(56)@paSi y (c) Au(87)@paSi. Los puntos de mayor composición de Si representan las PA-BU' Si nps solas $\left(\Phi / \Phi_{0}=1\right)$.

Los estudios se realizaron por agregado de volúmenes conocidos de Si nps sobre una muestra de Au nps, se agitaron vigorosamente por 15 min y luego se midió la absorbancia de la muestra, para finalmente ser ajustada tal que $A_{341}$ resulte entre 0.05 y 0.1 , y finalmente medir el espectro de emisión excitando a $341 \mathrm{~nm}$. Los agregados se realizaron hasta la formación de precipitados, lo cual puede notarse en las figuras (b) y (c), donde la caída en $\Phi$ es notoria. En principio, podemos diferenciar regiones de quenching y de aumento, según la composición del sistema. Mientras que para los ensamblados blanco $\Phi$ disminuyó siempre, para los ensamblados sistema se observó un aumento en el caso de las Au nps más grandes. En la figura 7.9 se presenta el comportamiento observado para estos últimos. Sin embargo, en los ensamblados estables en suspensión se observa un aumento en $\Phi$ de hasta el 100\%. El hecho de que el aumento se de en ensamblados con Au nps de mayor tamaño es coherente con el hecho de que en un proceso de MEF, donde la contribución dispersiva del coeficiente de extinción del metal es la responsable del aumento en el campo eléctrico local [23][24]. Es importante que al aumentar la composición de Si nps el efecto disminuye y coincide con la observación de un precipitado en la solución, lo cual es un claro indicativo del rol de mediador de las Si nps en la formación de aglomerados de Au nps.

Un efecto observable en el caso de MEF es la reducción del tiempo de vida del estado excitado que interactúa con la superficie metálica, que pudimos observar usando medidas de emisión resuelta en el tiempo. Se adquirió a múltiples longitudes de inda de detección, para mejorar la calidad del ajuste del modelo, adquiriendo espectros resueltos en el tiempo (TRES, del inglés Time Resolved Spectroscopy). Al momento de analizar las trazas obtenidas, se observó efectivamente la reducción del tiempo de vida medio para los ensamblados sistema, mientras que para los ensamblados blanco se mantuvo constante, dentro del error experimental. La variación en $\Phi$ fue acompañada con una reducción en el tiempo, ya sea que $\Phi$ haya aumentado o disminuido, lo cual indica que el mecanismo operativo, tanto en la región de quenching como de aumento de la emisión, implica el 
aumento en la cantidad de vías de desactivación del estado emisor. A su vez, es importante notar que en la región de aumento fue necesario considerar un componente más para el modelo de ajuste. Este cambio puede interpretarse como una heterogeneidad en el estado fundamental, que puede racionalizarse como fluoróforos localizados en distintos huecos del arreglo tridimensional del ensamblado. A su vez, la consideración de este componente extra hace que los tiempos de decaimiento $\mathrm{T}_{1}$ y $\mathrm{T}_{3}$ aumenten con respecto a los medidos para PABU' Si nps solas. Es muy posible que este efecto sea un artefacto del ajuste más que un aumento real, ya que el tiempo promediado por amplitudes $\langle\mathrm{T}>$ disminuye con el aumento la composición de $\mathrm{Si}$, en los casos donde fue necesario cambiar el modelo de ajuste.
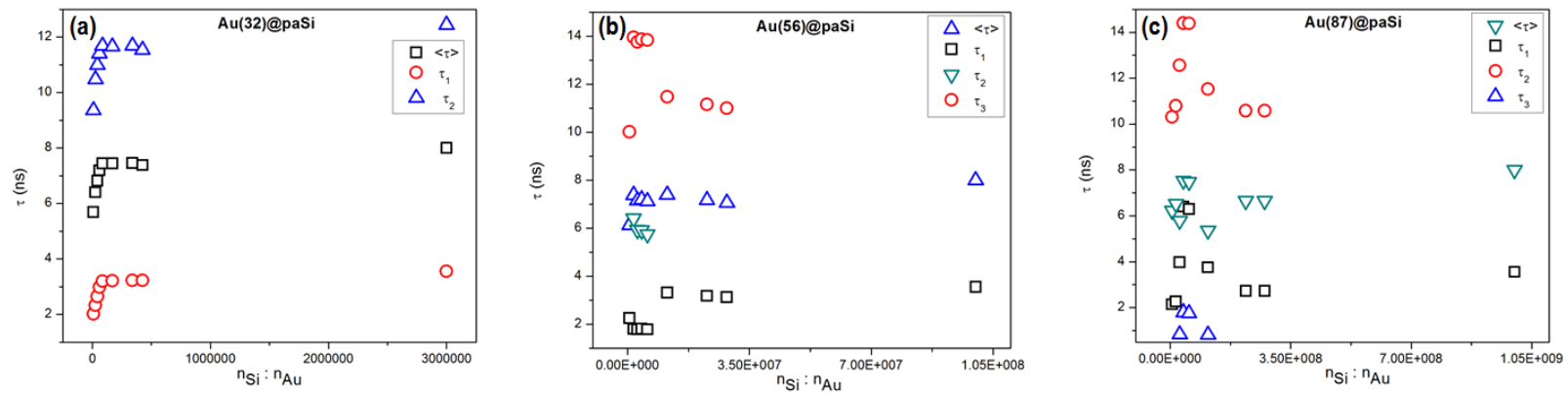

Figura 7.10. Variación de los tiempos de vida (considerando como referencia PA-BU' Si nps) para (a) Au(32)@paSi, (b) Au(56)@paSi y (c) Au(87)@paSi. Los puntos de mayor composición de Si representan las PA-BU' Si nps solas.

Los espectros asociados a cada tiempo de decaimiento considerando un modelo paralelo (DAS, del inglés Decay Associated Spectra) se presentan en la figura 7.11, y se asemejan a los obtenidos para PA-BU' Si nps, salvando el hecho de la consideración de un DAS extra, lo cual constituye un hecho significativo. Los tiempos de decaimiento se encuentran entre 1.5 y 4 ns (DAS 1) para la componente más corta, 9 y 12 ns para la componente larga (DAS 2), mientras que la componente extra necesaria aparece cerca de 6 ns (DAS 3). En la figura 7.10 se muestra la evolución de los т y de <T> en función de la composición del ensamblado.
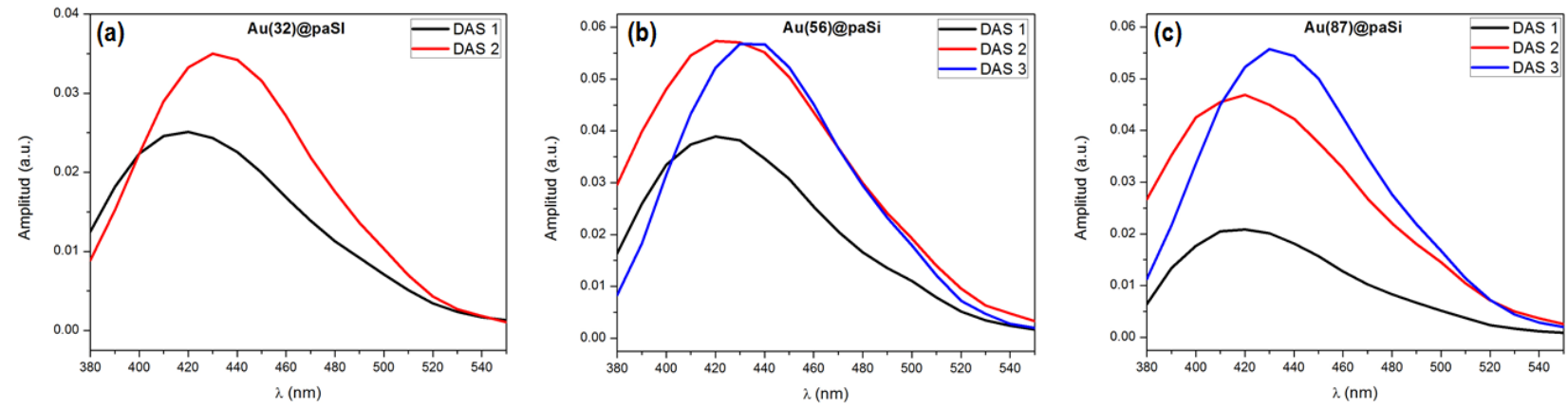

Figura 7.11. DAS obtenidos en el análisis de las medidas de TRES usando un modelo paralelo. Los espectros corresponden a (a) Au(32)@paSi, (b) Au(56)@paSi y (c) Au(87)@paSi.

Si bien todas las medidas colectadas apuntan a una interacción clara entre Au nps y PA-BU' Si nps que puede conllevar a quenching o MEF, según el grado de aglomeración del ensamblado, para obtener mayor información sobre la dinámica del estado emisor se realizaron experimentos con luz polarizada. La posible migración de energía de excitación en los procesos (ya sea por quenching bimolecular, MEF o quenching por modos de oscilación superiores) quedan en evidencia en la despolarización de la emisión a mayor velocidad, comparado al fluoróforo aislado (figura 7.12). 


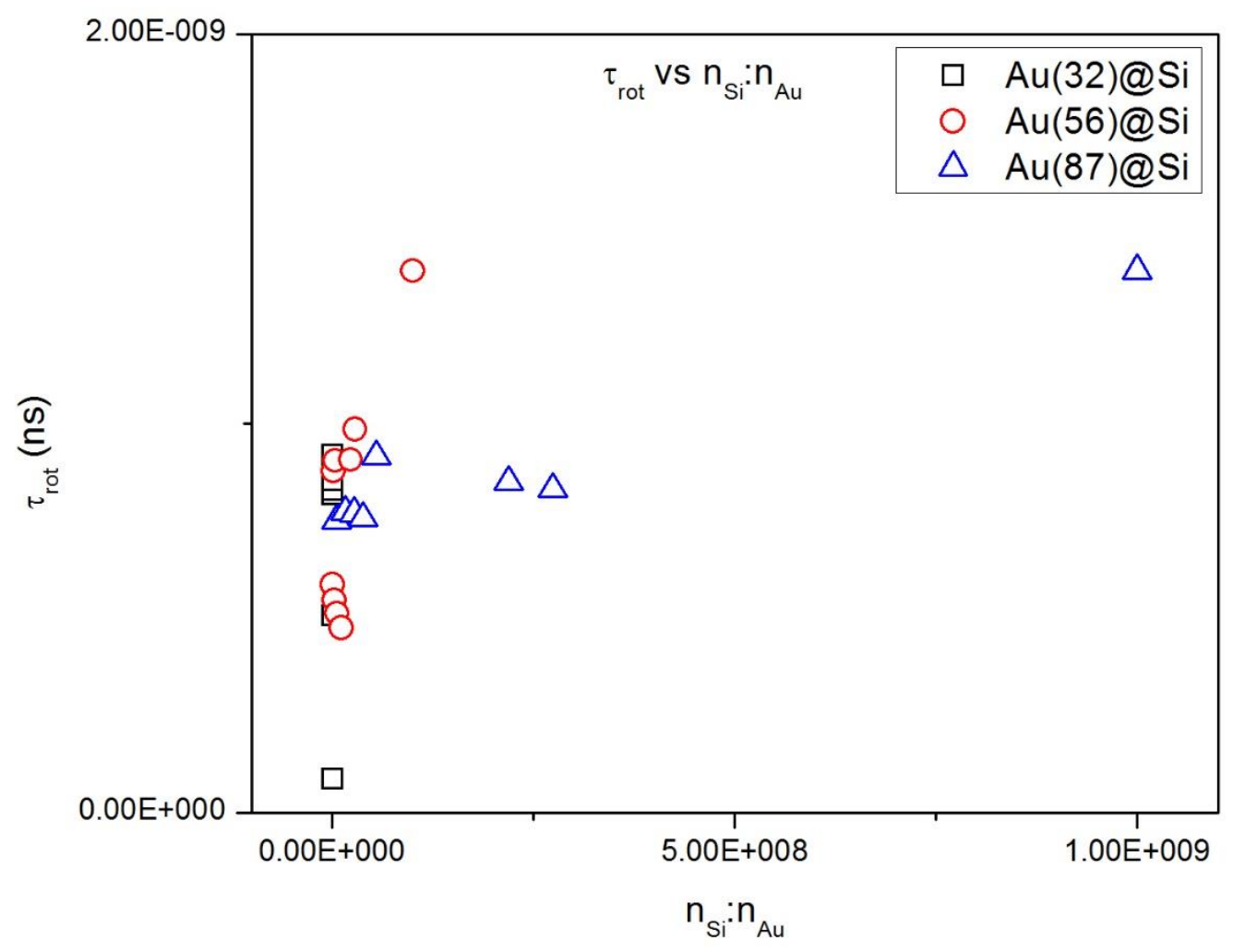

Figura 7.12. Variación de los tiempos de despolarización en ensamblados Au@Si.

\subsubsection{Generación de especies reactivas de oxígeno: Oxígeno Singlete $\mathrm{O}_{2}\left({ }^{1} \Delta_{g}\right)$.}

Como se discutió en la sección previa, se probó que la presencia de Au nps cambia la población y dinámica del estado excitado que domina la emisión de las Si nps. Este hecho podría tener efecto sobre toda la fotoquímica del estado excitado emisor, que en el caso de Si nps es el responsable de la formación de $\mathrm{O}_{2}\left({ }^{1} \Delta_{\mathrm{g}}\right)$. Con esta idea en mente, se evaluó la producción de esta especie reactiva con los coloides que presentaron un aumento en Ф. Para esto, se realizaron medidas de fosforescencia a $1270 \mathrm{~nm}$ a fin de cuantificar la cantidad de $\mathrm{O}_{2}\left({ }^{1} \Delta_{g}\right)$ producido por dispersiones coloidales en agua deuterada. Se excitó a $355 \mathrm{~nm}$ con el tercer armónico de un laser de Nd:YAG, trabajando a distintas energías de pulso, para extrapolar el comportamiento a e energías tendiendo a cero. Finalmente, se empleó TMPyP como fotosensibilizador referencia para la longitud de onda de excitación y el medio de trabajo [25], [26]. Los valores obtenidos usando esta técnica se resumen en la siguiente figura.

$$
\begin{array}{lll}
\mathrm{S}^{*}+3 \mathrm{O}_{2} \rightarrow \mathrm{S}+1 \mathrm{O}_{2} & \frac{\Phi_{\Delta, X}}{\Phi_{\Delta, R}}=\left(\frac{I_{\Delta, X}^{t=0}}{I_{\Delta, R}^{t=0}}\right) & \Phi_{\Delta, \mathrm{Si}}=(2.0 \pm 0.5) * 10-3 \\
1 \mathrm{O}_{2} \rightarrow 3 \mathrm{O}_{2}+\mathrm{hv} & \Phi_{\Delta, \mathrm{Au}(68) @ \mathrm{Si}}=0.12 \pm 0.03 \\
& \Phi_{\Delta, \mathrm{Au}(110) @ \mathrm{Si}}=0.32 \pm 0.05
\end{array}
$$

Figura 7.13. rendimientos cuánticos de formación de $\mathrm{O}_{2}\left({ }^{1} \Delta_{\mathrm{g}}\right) \Phi_{\Delta}$. 
Este cambio en los rendimientos cuánticos de generación de oxígeno singlete también fue reportado para distintos sistemas híbridos con componentes metálicos [3], [7], [27], [28]. Sin embargo, el mecanismo por el cual aumenta no está claro. Al menos existen 2 posibilidades distintas: Puede haber un cambio en el rendimiento cuántico de emisión del $\mathrm{O}_{2}\left({ }^{1} \Delta_{\mathrm{g}}\right)$ (Teniendo en cuenta que detectamos a través de la emisión a $1270 \mathrm{~nm}$ ) o un aumento en la población del estado excitado que genera $\mathrm{O}_{2}\left({ }^{1} \Delta_{\mathrm{g}}\right)$. Para poder diferenciar la influencia de ambos procesos es necesario utilizar una vía alternativa de detección, como es el uso de una sonda reactiva específica para $\mathrm{O}_{2}\left({ }^{1} \Delta_{\mathrm{g}}\right)$ como es el Sensor Green ${ }^{\circledR}$.

\subsection{Conclusiones}

En este capítulo se discutieron los resultados obtenidos en la preparación de ensamblados coloidales entre Au nps y Si nps, con el objetivo de regular las propiedades fotofíscas y fotoquímicas del fluoróforo base. A tal fin, se sintetizaron Au nps de distintos tamaños usando el método de crecimiento de semillas y Si nps por oxidación de siliciuros metálicos (Capítulo 5). Estos bloques de construcción se caracterizaron usando diversas técnicas y se estudiaron sus propiedades ópticas. Se encontró que el agregado de PA-BU' Si nps induce la aglomeración del coloide de Au, que con lleva a la formación de ensamblados coloidales con estabilidad limitada, pero con propiedades ópticas regulables, a través de procesos de quenching o de MEF. En particular, se encontró un aumento razonable para los rendimientos cuánticos de fotoluminiscencia $\Phi_{\mathrm{PL}}$ usando Au nps de 56 y $87 \mathrm{~nm}$ de diámetro. Con estos ensamblados se estudió la posibilidad de mejorar las propiedades de las Si nps como fotosensibilizadores de $\mathrm{O}_{2}\left({ }^{1} \Delta_{g}\right)$, obteniéndose aumentos importantes. Sin embargo, para poder utilizar estos ensamblados en futuros desarrollos es imperativo continuar trabajando para elucidar el mecanismo involucrado en el aumento de producción de $\mathrm{O}_{2}\left({ }^{1} \Delta_{\mathrm{g}}\right)$ y optimizar su estabilidad coloidal.

Finalmente, los resultados obtenidos en esta parte pueden resumirse del siguiente modo:

- Síntesis de bloques de construcción empleando precursores de distintas naturalezas.

- Preparación de ensamblados con nuevas propiedades ópticas.

- Controlando la química y estructura de los bloques pueden obtenerse propiedades regulables.

- Capacidad de fotosensibilización sintonizable.

\subsection{Referencias}

[1] P. V. Kamat, "Meeting the clean energy demand: Nanoestructure Architectures for Solar Energy Conversion," Phys. Chem., vol. 392, pp. 2834-2860, 2007.

[2] K. L. Kelly, E. Coronado, L. L. Zhao, and G. C. Schatz, "Feature article," J. Phys. Chem. B, vol. 107, pp. 668-677, 2003.

[3] M. B. Rivas Aiello, J. J. Romero, S. G. Bertolotti, M. C. Gonzalez, and D. O. Mártire, "Effect of Silver Nanoparticles on the Photophysics of Riboflavin: Consequences on the ROS Generation," J. Phys. Chem. C, p. acs.jpcc.6b06385, 2016.

[4] L. Maretti, P. S. Billone, Y. Liu, and J. C. Scaiano, "Facile photochemical synthesis and characterization of highly fluorescent silver nanoparticles," J. Am. Chem. Soc., vol. 131, no. 28, pp. 13972-13980, 2009.

[5] M. L. Marin, K. L. McGilvray, and J. C. Scaiano, "Photochemical strategies for the synthesis of gold nanoparticles from $\mathrm{Au}(\mathrm{III})$ and $\mathrm{Au}(\mathrm{I})$ using photoinduced free radical generation," J. Am. Chem. Soc., vol. 130, no. lii, pp. 16572-16584, 2008.

[6] K. L. McGilvray, M. R. Decan, D. Wang, and J. C. Scaiano, "Facile photochemical synthesis of 
unprotected aqueous gold nanoparticles," J. Am. Chem. Soc., vol. 128, pp. 15980-15981, 2006.

[7] K. Aslan, J. R. Lakowicz, and C. D. Geddes, "Metal-enhanced fluorescence using anisotropic silver nanostructures: Critical progress to date," Anal. Bioanal. Chem., vol. 382, pp. 926-933, 2005.

[8] W. Haiss, N. T. K. Thanh, J. Aveyard, and D. G. Fernig, "Determination of Size and Concentration of Gold Nanoparticles from UV - Vis Spectra Determination of Size and Concentration of Gold Nanoparticles from UV - Vis Spectra," vol. 79, no. October, pp. 4215-4221, 2015.

[9] D. Neiner, H. W. Chiu, and S. M. Kauzlarich, "Low-temperature solution route to macroscopic amounts of hydrogen terminated silicon nanoparticles," J. Am. Chem. Soc., vol. 128, pp. 11016-11017, 2006.

[10] R. K. Baldwin, K. a. Pettigrew, J. C. Garno, P. P. Power, G. Y. Liu, and S. M. Kauzlarich, "Room temperature solution synthesis of alkyl-capped tetrahedral shaped silicon nanocrystals," J. Am. Chem. Soc., vol. 124, pp. 1150-1151, 2002.

[11] K. Pettigrew and Q. Liu, "Solution synthesis of alkyl-and alkyl/alkoxy-capped silicon nanoparticles via oxidation of Mg2Si,” Chem. ..., no. 31, pp. 4005-4011, 2003.

[12] J. Turkevich, P. C. Stevenson, and J. Hillier, "A study of the nucleation and growth processes in the synthesis of colloidal gold," Discuss. Faraday Soc., vol. 11, no. c, pp. 55-75, 1951.

[13] W. Haiss, N. T. K. Thanh, J. Aveyard, and D. G. Fernig, "Determination of Size and Concentration of Gold Nanoparticles from UV - Vis Spectra Determination of Size and Concentration of Gold Nanoparticles from UV - Vis Spectra," no. October, pp. 1-7, 2015.

[14] K. R. Brown and M. J. Natan, "Hydroxylamine seeding of colloidal Au nanoparticles in solution and on surfaces," Langmuir, vol. 14, no. 4, pp. 726-728, 1998.

[15] T. Kim, C. H. Lee, S. W. Joo, and K. Lee, "Kinetics of gold nanoparticle aggregation: Experiments and modeling," J. Colloid Interface Sci., vol. 318, no. 2, pp. 238-243, 2008.

[16] S. K. Ghosh and T. Pal, "Interparticle Coupling Effect on the Surface Plasmon Resonance of Gold Nanoparticles: From Theory to Applications," Chem. Rev., vol. 107, no. 11, pp. 4797-4862, 2007.

[17] A. N. Shipway, M. Lahav, R. Gabai, and I. Willner, "Investigations into the electrostatically induced aggregation of Au nanoparticles," Langmuir, vol. 16, no. 23, pp. 8789-8795, 2000.

[18] J. C. Azcárate, G. Corthey, E. Pensa, C. Vericat, M. H. Fonticelli, R. C. Salvarezza, and P. Carro, "Understanding the surface chemistry of thiolate-protected metallic nanoparticles," J. Phys. Chem. Lett., vol. 4, pp. 3127-3138, 2013.

[19] G. Corthey, L. J. Giovanetti, J. M. Ramallo-López, E. Zelaya, A. A. Rubert, G. A. Benitez, F. G. Requejo, M. H. Fonticelli, and R. C. Salvarezza, "Synthesis and characterization of gold@gold(I)thiomalate core@shell nanoparticles," ACS Nano, vol. 4, no. 6, pp. 3413-3421, 2010.

[20] A. Kumar, S. Mandal, P. R. Selvakannan, R. Pasricha, A. B. Mandale, and M. Sastry, "Investigation into the interaction between surface-bound alkylamines and gold nanoparticles," Langmuir, vol. 19, no. 15, pp. 6277-6282, 2003.

[21] M. Aslam, L. Fu, M. Su, K. Vijayamohanan, and V. P. Dravid, "Novel one-step synthesis of aminestabilized aqueous colloidal gold nanoparticles," J. Mater. Chem., vol. 14, no. 12, p. 1795, 2004.

[22] J. R. Lakowicz, M. H. Chowdury, K. Ray, J. Zhang, Y. Fu, R. Badugu, C. R. Sabanayagam, K. 
Nowaczyk, H. Szmacinski, K. Aslan, and C. D. Geddes, "Plasmon-controlled fluorescence: a new detection technology," Proc. SPIE 6099, Plasmon. Biol. Med. III, vol. 6099, pp. 609909-1-15, 2006.

[23] J. R. Lakowicz, "Radiative decay engineering 5: Metal-enhanced fluorescence and plasmon emission," Anal. Biochem., vol. 337, pp. 171-194, 2005.

[24] Y. Zhang, K. Aslan, M. J. R. Previte, and C. D. Geddes, "Metal-enhanced fluorescence: Surface plasmons can radiate a fluorophore's structured emission," Appl. Phys. Lett., vol. 90, pp. 1-3, 2007.

[25] P. R. Ogilby and C. S. Foote, "Chemistry of singlet oxygen. 36. singlet molecular oxygen (1deltag) luminescence in solution following pulsed laser excitation. solvent deuterium isotope effects on the lifetime of singlet oxygen.," J. Am. Chem. Soc., vol. 104, pp. 2069-2070, 1982.

[26] P. Ogilby and C. Foote, "Chemistry of singlet oxygen. 42. Effect of solvent, solvent isotopic substitution, and temperature on the lifetime of singlet molecular oxygen (1. DELTA. g)," J. Am. Chem. Soc., vol. 103, pp. 3423-3430, 1981.

[27] K. Aslan, M. Wu, J. R. Lakowicz, and C. D. Geddes, "Kadir Aslan, † Meng Wu, ‡,§ Joseph R. Lakowicz, $\ddagger$ and Chris D. Geddes* ,†, $\ddagger$," pp. 1524-1525, 2007.

[28] K. Ray, R. Badugu, and J. R. Lakowicz, "Metal-Enhanced Fluorescence from CdTe Nanocrystals: A Single-MoleculelrFluorescence Study," J. Am. Chem. Soc., vol. 128, pp. 8998-8999, 2006. 
- 162 - 
Parte Final 
- 164 - 


\section{Capítulo 8. Conclusiones Generales}

\subsection{Conclusiones}

En el presente trabajo tomamos como hipótesis de partida que las Si nps poseen la capacidad de actuar como eficientes dadores y/o aceptores de energía/carga. Para desarrollar esta hipótesis, planteamos los siguientes objetivos:

- Sintetizar, caracterizar y evaluar la estabilidad en suspensión acuosa y en solventes orgánicos, de Si nps conjugados con diversos colorantes orgánicos y con nanopartículas de oro.

- Investigar la dinámica de los procesos físicos y químicos, en particular aquellos que involucran transferencia de carga o energía.

- Evaluar la eficiencia de la transferencia de energía o carga en función de la naturaleza del aceptor (dador), el número de aceptores (dadores) de energía unidos a las Si nps, la constante de decaimiento radiativo y no radiativo del dador de energía, tamaño de partículas y forma.

A tal fin, sintetizamos una variedad de Si nps las cuales fueron caracterizadas aplicando diversas técnicas y posteriormente se estudiaron en detalle sus propiedades fotofísicas, prestando atención a la dinámica de los estados excitados involucrados en el proceso de emisión. Las técnicas empleadas y las estrategias sintéticas abordadas en este trabajo se discutieron en detalle en los capítulos 2 y 3.

En el capítulo 4, prestamos atención a las propiedades de Si nps ultra pequeñas, y el rol activo que cumple la superficie en el proceso de emisión. La dependencia de la PL de Si nps con el tamaño, la cristalinidad, la estructura y la química superficial constituye un desafío importante al momento de diseñar sensores y fotosensibilizadores. A su vez, la baja estabilidad de Si nps $\mathrm{H}$ terminales conlleva a procesos de oxidación no deseados, a menos que los procesos de síntesis y modificación sean realizados en estrictas condiciones de ausencia de oxígeno y en suspensiones de solventes orgánicos bien secos y de alta pureza. Nuestros resultados muestran que las propiedades $\mathrm{PL}$ de Si nps de 1-2nm oxidadas de forma controlada que presentan estructuras del tipo Si-O-Si son independientes de la estrategia sintética (TD o BU) y de la derivatización postsíntesis con moléculas orgánicas con distintos grupos funcionales. Esta observación tiene importantes consecuencias para el desarrollo de los usos tecnológicos de las Si nps, ya que la ruta sintética a elegir puede seleccionarse a partir de costos y disponibilidad de reactivos o equipamiento. Incluso aunque las principales vías de síntesis requieren la minimización de oxígeno disuelto en el medio, el uso posterior de reacciones de silanización para generar una cobertura orgánica puede realizarse en atmósfera de aire y a temperatura ambiente. Como resultado, se puede derivatizar la superficie mediante uniones químicas con moléculas biocompatibles sin que se observen cambios en las propiedades de PL. Estas últimas propiedades son importantes para la dirección específica a blancos y prevenir la opsonización en aplicaciones en censado.

En el capítulo 5 nos enfocamos en las propiedades ópticas en función del tamaño para Si nps sintetizadas por vías de abajo hacia arriba, que presentan emisión en la región azul-verde del espectro visible (banda $\mathrm{F}$ ). Sintetizamos exitosamente Si nps por oxidación de fases de Zintl en fase orgánica, que posteriormente fueron cubiertas con 2-propenilamina que denominamos PA-BU' Si nps. Estas nanopartículas se caracterizaron empleando HR-TEM, FTIR y XPS. Variando las condiciones de síntesis logramos controlar el tamaño de las $\mathrm{Si}$ nps obtenidas, lo cual permitió sintonizar ciertas propiedades ópticas y electroquímicas. A través de experimentos ópticos de TCSPC y TAS, pudimos establecer que si bien los estados involucrados en el proceso de absorción pueden controlarse ajustando las condiciones sintéticas, los estados emisores suelen estar asociados a trampas de 0 o N. Si bien la emisión desde estas trampas se ve modificada levemente según el 
tamaño de las Si nps, la distribución de trampas superficiales relacionadas al cubrimiento con $\mathrm{N}$ y $\mathrm{O}$, juega un papel importante.

El método sintético desarrollado en este capítulo fue utilizado en el resto de los experimentos involucrados en este trabajo de tesis, debido a su alta reproducibilidad y fundamentalmente por la simpleza con la cual pueden obtenerse cantidades en el orden de los mg de partículas, lo cual es fundamental para experimentos ópticos de absorción de transientes en los fs.

En los capítulos 6 y 7 se ensayaron distintas alternativas para regular las propiedades ópticas de las Si nps, a fin de convertirlas en sistemas más versátiles con posibles aplicaciones en censado, conversión de energía 0 como fotosensibilizadores.

En el capítulo 6 comentamos sobre la preparación de diversas díadas compuestas por colorantes orgánicos y Si nps. Estas díadas fueron sometidas a un proceso de purificación lo más exhaustivo posible, para evitar la interferencia de cromóforos libres, sin embargo esta tarea fue muy difícil de llevar adelante. Se eligió el método que mejores resultados dio, pero que aún dista de ser el óptimo. Las propiedades ópticas de estos ensamblados fueron estudiadas en detalle usando una batería de técnicas espectroscópicas las cuales permitieron obtener información útil para esclarecer la dinámica de los estados excitados involucrados, en particular la participación y decaimiento de estados de transferencia de carga. Estos últimos fueron interpretados en términos de la teoría de Marcus, observando que en general el proceso de separación de cargas se encontraba en la región normal, salvo en el caso de díadas conteniendo carotenoides que se encuentran en la región inversa. Los resultados de este capítulo constituyen una base fotoquímica para la potencial utilización de estas díadas en fotoelectrodos de gran área superficial, útiles en conversión de energía solar y en celdas de combustible fotoelectroquímicas.

En el capítulo 7 se discutieron los resultados obtenidos en la preparación de ensamblados coloidales entre Au nps y Si nps, con el objetivo de regular las propiedades fotofísicas y fotoquímicas del fluoróforo base. A tal fin, se sintetizaron Au nps de distintos tamaños usando el método de crecimiento de semillas y Si nps por oxidación de siliciuros metálicos que se estudió detalladamente en el capítulo 5. Estos bloques de construcción se caracterizaron usando diversas técnicas y se estudiaron sus propiedades ópticas. Se encontró que el agregado de PA-BU' Si nps induce la aglomeración del coloide de Au, que conlleva a la formación de ensamblados coloidales con estabilidad limitada, pero con propiedades ópticas regulables, a través de procesos de quenching 0 de MEF. En particular, se encontró un aumento razonable para los rendimientos cuánticos de fotoluminiscencia $\Phi_{\mathrm{PL}}$ usando Au nps de 56 y $87 \mathrm{~nm}$ de diámetro. Con estos ensamblados se estudió la posibilidad de mejorar las propiedades de las Si nps como fotosensibilizadores de $\mathrm{O}_{2}\left({ }^{1} \Delta_{g}\right)$, obteniéndose aumentos importantes. Sin embargo, para poder utilizar estos ensamblados en futuros desarrollos es imperativo continuar trabajando para elucidar el mecanismo involucrado en el aumento de producción de $\mathrm{O}_{2}\left({ }^{1} \Delta_{g}\right)$ y optimizar su estabilidad coloidal.

Finalmente, a lo largo del presente trabajo de tesis pudo demostrarse la factibilidad de usar a las Si nps como parte de díadas en las cuales se transfiera carga, permitiendo así regular sus propiedades ópticas. Particularmente, logramos obtener sistemas basados en Si nps en los cuales la obtención de especies de carga separada se da por excitación directa en el visible, superando un inconveniente muy importante frente a las $\mathrm{Si}$ nps. Esto constituye un paso más en el desarrollo de estrategias racionales para el diseño de sistemas nanoestructurados funcionales para aplicaciones en censado, conversión de energía terapéutica y usos biológicos.

\subsection{Perspectivas y trabajo futuro}

Los resultados obtenidos a lo largo de esta tesis, constituyen un punto de partida para el desarrollo de sistemas basados en Si nps. Por un lado, abordamos un amplio trabajo sintético que permitió encontrar vías sintéticas acordes a las distintas necesidades del sistema futuro a preparar, logrando obtener de forma sencilla, Si nps con propiedades luminiscentes muy estables frente a cambios en el entorno. Con estas Si nps se desarrollaron 
diversos sistemas en los cuales las propiedades ópticas pueden ser reguladas al momento de elegir sus bloques de construcción.

Los pasos a seguir a fin de profundizar el trabajo, serían en dos direcciones fundamentalmente:

- Por un lado, continuar con los estudios espectroscópicos en los ensamblados coloidales Au@Si descriptos en el capítulo 7 y en las díadas del capítulo 6 . Si bien se realizó un avance importante, quedan aspectos en los cuales puede profundizarse aún más. En el caso de díadas, a través de los estudios realizados no podemos concluir nada sobre la naturaleza del estado al cual relaja el estado de carga separada formado. Este proceso involucra probablemente la recombinación del par, pudiendo conducir a productos de distintos estados de spin. Para obtener mayor información, deberían realizarse estudios de TAS en los $\mu s$ o de EPR (Electron Paramagnetic Resonance) que quedaron por fuera de esta tesis.

- Por el otro, con los conocimientos aprendidos sobre díadas basadas en Si nps pueden diseñarse fotoelectrodos útiles para conversión de energía solar, ya sea utilizando las díadas empleadas 0 variando los colorantes. En esta dirección es necesario realizar estudios electroquímicos para obtener mayor información sobre las díadas a emplear y los procesos de inyección de carga a los electrodos.

\subsection{Lugar de trabajo y trabajo en colaboración}

Gran parte de los resultados presentados en esta tesis se obtuvieron en el Laboratorio de Especies Altamente Reactivas del Instituto de Investigaciones Fisicoquímicas Teóricas y Aplicadas (INIFTA). Sin embargo, una parte importante de ellos son producto de distintas estadías en grupos colaboradores en el exterior. Los laboratorios en los cuales desarrollamos diversos experimentos fueron:

- Friedrich-Alexander Universität (Erlangen - Nürnberg), Department Chemie und Pharmazie; trabajando bajo la supervision de la Prof. Dr. Carola Kryschi.

- University of California at Riverside, Department of Bioengineering; trabajando bajo la supervisión del Prof. Dr. Valentine I. Vullev.

- Arizona State University, Department of Chemistry and Biochemistry; trabajando bajo la supervisión de la Prof. Dr. Ana L. Moore.

Gracias al trabajo conjunto con dichos laboratorios publicamos distintos trabajos en revistas científicas internacionales y establecimos nuevos proyectos de colaboración.

\subsection{Trabajos publicados}

- Versatile silicon nanoparticles with potential uses as photoluminiscent sensors and photosensitizers. Paula Caregnato, María Laura Dell'Arciprete, Mónica C. Gonzalez, Cristian R. Lillo, Hernán B. Rodríguez and Juan J. Romero. In Photochemistry: Volume 44 (Specialist Periodical Reports); Angelo Albini and Elisa Fasani, Eds.; Royal Society of Chemistry, 2016; pp 324-347, Print ISBN: 978-1-78262543-8.

- "On the efficacy of anthracene isomers for triplet transmission from CdSe nanocrystals" P. Xia, Z. Huang, X. Li, J. J. Romero, V. I. Vullev, G. S. H. Pau and M. L. Tang, Chem. Commun., Chem. Commun., 53, 1241-1244 (2017) DOI: 10.1039/C6CC08229G.

- "Gating That Suppresses Charge Recombination-The Role of Mono-N-Arylated diketopyrrolopyrrole". Anna Purc, Eli M. Espinoza, Rashid Nazir, Juan J. Romero, Kamil Skonieczny, Artur Jezewski, Jillian M. Larsen, Daniel T. Gryko and Valentine I. Vullev. Journal of the American Chemical Society 138(39), 12826-12832 (2016). DOI: 10.1021/jacs.6b04974.

- Effect of Silver Nanoparticles on the Photophysics of Riboflavin: Consequences on the ROS Generation". María Belén Rivas Aiello, Juan José Romero, Sonia G. Bertolotti, Mónica C. Gonzalez 
and Daniel O. Mártire. Journal of Physical Chemistry C 120(38), 21967-21975 (2016). DOI: 10.1021/acs.jpcc.6b06385.

- "Polyethylene glycol-coated blue-emitting silicon dots with improved properties for uses in aqueous and biological environments". Damián Rodríguez Sartori, Cristian R. Lillo, Juan J. Romero, María Laura Dell'Arciprete, Alejandro Miñán, Mónica Fernández Lorenzo de Mele and Mónica C. Gonzalez. Nanotechnology 27(47), 475704 (2016). DOI: 10.1088/0957-4484/27/47/475704.

- "Impact of Iron Doping on 2 - 4 nm Size Silicon Nanoparticles Properties". Juan J. Romero, Marc Wegmann, Hernán B. Rodríguez, Cristian R. Lillo, Aldo A. Rubert, Stefanie Klein, Mónica L. Kotler, Carola Kryschi and Mónica C. González. J. Phys. Chem. C, 119, 5739-5746, 2015.

- "Organic-Coating of 1-2 nm Size Silicon Nanoparticles: Effect on the Particle Properties". Cristian R. Lillo, Juan J. Romero, Manuel Llansola Portolés, Reinaldo Pis Diez, Paula C. Caregnato, and Mónica C. Gonzalez. Nano Research (DOI 10.1007/s12274-015-0716-z 2015).

- "Photoluminescent 1-2 nm Sized Silicon Nanoparticles: A Surface-Dependent System". Juan J. Romero, Manuel J. Llansola Portolés, María L. Dell' Arciprete, Hernán B. Rodríguez, Ana L. Moore, Mónica C. González. Chem. Mater., 2013, 25 (17), pp 3488-3498.

- "Understanding the Parameters Affecting the Photoluminescence of Silicon Nanoparticles". Manuel J. Llansola Portolés, Reinaldo Pis Diez, María L. Dell' Arciprete, Paula Caregnato, Juan J. Romero, Daniel O. Mártire, Omar Azzaroni, Marcelo Ceolín, Mónica C. González. J. Phys. Chem. C, 2012, 116 (20), pp 11315-11325. 
- 169 - 


\section{Glosario}

A continuación presentamos un glosario con siglas y abreviaturas de uso frecuente, para facilitar la lectura de este trabajo.

Si nps: nanopartículas de Silicio.

PSi: Silicio Poroso.

TD Si nps: Si nps sintetizadas por anodización de cristales de Si.

TD' Si nps: Si nps sintetizadas por grabado con HF sobre PSi.

BU Si nps: Si nps sintetizadas por reducción en micelas inversas.

BU' Si nps: Si nps sintetizadas por oxidación de siliciuros metálicos.

S1: Si nps sintetizadas por oxidación de siliciuros metálicos de (3.6 \pm 0.4$) \mathrm{nm}$.

S3: Si nps sintetizadas por oxidación de siliciuros metálicos de $(4 \pm 1) \mathrm{nm}$.

S4: Si nps sintetizadas por oxidación de siliciuros metálicos de $(3.6 \pm 0.4) \mathrm{nm}$.

PA (SM) Si nps: Si nps sintetizadas por el método correspondiente según las siglas, cubiertas con propilamina.

PASiOx (SM) Si nps: Si nps sintetizadas por el método correspondiente según las siglas, cubiertas con 3aminopropil-trietoxisilano.

MASiOx(SM) Si nps: Si nps sintetizadas por el método correspondiente según las siglas, cubiertas con metacrilato de metilo.

1: Derivado monocarboxilado de la tetra-mesitil porfirina, TMP.

1': Derivado monocarboxilado de la tetra-mesitil porfirina con un átomo central de Zn, Zn(TMP).

dpp: Derivado asimétrico del dicetopirrolopirrol, con un grupo octilo y un grupo carboxilo en cada $\mathrm{N}$ del núcleo pirrolopirrol.

C9: Derivado monocarboxilado del caroteno con 9 dobles enlaces.

C10: Derivado monocarboxilado del caroteno con 10 dobles enlaces.

C11: Derivado monocarboxilado del caroteno con 11 dobles enlaces.

Las estructuras de los colorantes empleados son las siguientes: 

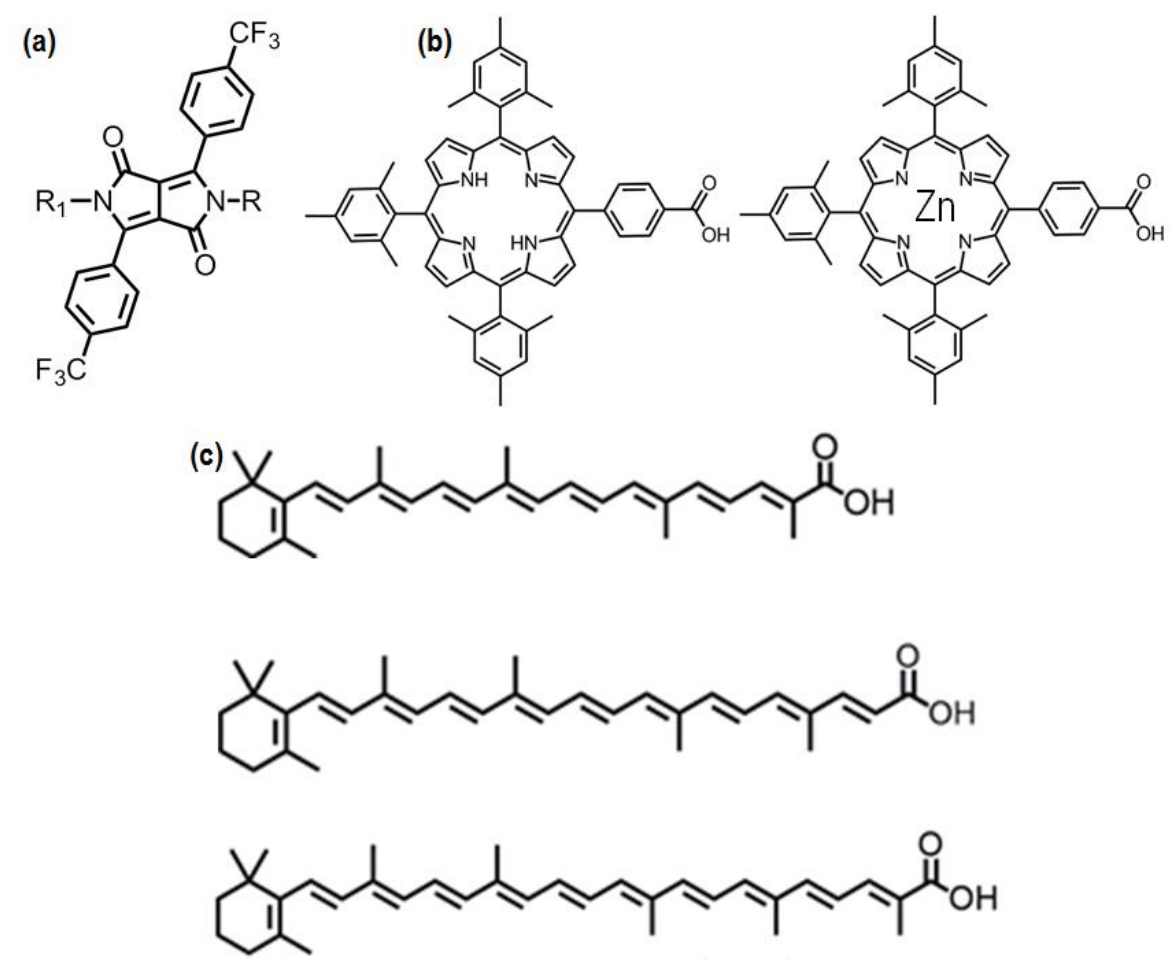

Figura G.1. Colorantes empleados en el capítulo 6. (a) dicetopirrolopirrol (dpp), el empleado en esta tesis presenta $\mathrm{R}_{1}=-\mathrm{COOH}$ y $\mathrm{R}=$ $\mathrm{C}_{8} \mathrm{H}_{17}$, (b) derivados carboxilados de tetra-mesitil porfirina libre y con $\mathrm{Zn}^{2+}$, (c) derivados carboxilados del caroteno con 9,10 y 11 dobles enlaces.

1paSj: Díada formada por el colorante 1 y las nanopartículas $S \mathrm{j}$, siendo $\mathrm{j}=1,304$.

1'paSj: Díada formada por el colorante 1' y las nanopartículas Sj, siendo j= 1, 3 o 4 .

dpppaSj: Díada formada por el colorante dpp y las nnopartículas $S \mathrm{j}$, siendo j=1, 3 o 4.

CkpaSj: Díada formada por el carotenoide de $k$ dobles enlaces $(k=9,10$ u 11) y las nanopartículas $S j$, siendo $j=1,304$.

EEM: Matrices de emisión excitación.

TCSPC: Conteo de fotones individuales correlacionados en el tiempo.

XPS: Espectroscopía fotoelectrónica de raxos $\mathrm{X}$.

TEM: Microscopía electrónica de transmisión.

HR-TEM: Micropscopía electrónica de alta resolución.

FTIR: Espectroscopía infrarroja por transformada de Fourier.

TAS: Espectroscopía de absorción de transientes resuelta en el femtosegundo.

DAS: Espectros asociados a decaimientos.

EAS: Espectros asociados evolución espectral.

SAS: Espectros asociados a especies. 
- 172 - 